\title{
Engineered delivery tools for gene therapy and optogenetics
}

\author{
Dissertation \\ for the award of the degree \\ "Doctor rerum naturalium" (Dr. rer. nat.) \\ of the Georg-August-University Göttingen \\ Within the doctoral program Sensory and Motor Neuroscience \\ of the Georg-August University School of Science (GAUSS) \\ submitted by
}

\section{Lara Timantra Schiller}

born in

Göttingen

Göttingen, February 2018 

Thesis committee members

Jens Gruber Ph.D.

Junior Research Group Medical RNA Biology

German Primate Center, Göttingen

\section{Prof. Dr. Stefan Treue}

Cognitive Neuroscience Laboratory

German Primate Center, Göttingen

\section{Camin Dean Ph.D.}

Trans-synaptic Signaling Group

European Neuroscience Institute, Göttingen

\section{Extended thesis committee members}

Dr. Manuela Schmidt

Somatosensory Signaling Group

Max Planck Institute for Experimentel Medicine, Göttingen

Dr. Alexander Hahn

Junior Research Group Herpesviruses

German Primate Center, Göttingen

\section{Prof. Dr. Ralf Heinrich}

Cellular Neurobiology Department

Schwann-Schleiden Research Center, Göttingen

Date of oral examination: 18.04.2018 



\section{Affidavit}

I hereby declare that the PhD thesis entitled "Engineered delivery tools for gene therapy and optogenetics" was written independently and with no other sources and aids than quoted.

Date

Lara Timantra Schiller 



\section{Acknowledgements}

I am most grateful to my supervisor Dr. Jens Gruber who gave me the opportunity to change my subject from plants to fascinating delivery tools. The freedom to make our own decisions and develop our skills gave us the possibility to learn not only from our success but also sustainably from failure. I am also grateful for his support and giving me time to co-organise two interesting conferences.

I would also like to thank my thesis committee members Prof. Dr. Stefan Treue and Dr. Camin Dean for their advice and feedback.

In particular, I am most thankful to Dr. Camin Dean and her lab members Anne Wolfes and Markus Stahlberg for providing me with methodological knowledge and materials. Without their advice it would have been much more difficult to set up my project. I thank Markus for assisting me with the patch clamp experiment.

Also my group members contributed with their knowledge and help to my work. I especially thank Kai Böker for assisting me with the NTA measurements, Rafael Rinaldi Ferreira for his scientific feedback, and Stefan Schneider for working hard in providing us with VLPs and sharing his protein purification experience. I am sincerely thankful for the numerous scientific input and discussions with Nicolas Lemus. Thank you for helping me to shape my ideas in a convincing way and great team-work on the experiments and data presentations of the exo-AAV paper. Thanks to Monica Cruz-Barrera for providing us the MSCs.

A special thanks goes to Angelina Schuder who introduced me to the lab routines and the rAAV production. I am most grateful to her and Ellen Eckermann-Felkl, Astrid Backhaus and Daniela Lazzarini for their technical and mental support. Thanks to Svenja Niehus for her fresh ideas and good work during lab rotation.

Thank you, Sarah Schlagowski, Andrea Koch, and Daniela Gerke for all the fun during the lunch breaks. I also thank Vladan Rankovic for the material support, performing the cochlea injection and the exchange of ideas about rAAV production.

Simone Luert, Larissa Hummel, and Nadine Schminke deserve a big thanks for having been so helpful and friendly showing me the staining techniques and the querulent electron microscope.

I thank Prof. A. Schneider and Dr. M. Kunadt for providing the NTA machine and tech- 
nical support and Prof. Dr. Henning Urlaub's lab for performing the mass spectrometry analysis.

I am most grateful to my great family and friends. Annika, we know each other since the first day at the university and how great is it that we are still in touch. And Johan, I am looking forward sharing my everyday life with you and everything we will experience. Mama, Papa, thank you for always supporting me when I need it the most and listening to my ideas.

This work was partly supported by the LifeScience-Stiftung and the Göttingen Graduate School for Neuroscience, Biophysics, and Molecular Biosciences (DFG Grant GSC 226/2). 


\section{Table of contents}

Affidavit . . . . . . . . . . . . . . . . . . . . . 1

Acknowledgements . . . . . . . . . . . . . . . . . 3

List of Figures . . . . . . . . . . . . . . . . . . . . . . . . . . 9

List of Tables . . . . . . . . . . . . . . . . . . . . 11

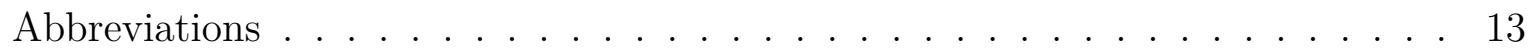

Abstract . . . . . . . . . . . . . . . . 17

1 Introduction $\quad 19$

1.1 Gene therapy . . . . . . . . . . . . . . . . . . . . . . . . . 19

1.1.1 Viral vectors in gene therapy . . . . . . . . . . . . . . . . 21

1.1.2 Optogenetics in research and for therapy . . . . . . . . . . . . . 22

1.2 Adeno-associated viruses . . . . . . . . . . . . . . . . . 24

Limitations of rAAVs for clinical approaches . . . . . . . . . 26

1.2.1 Exosome-associated AAVs . . . . . . . . . . . . . . . . 28

1.3 Lentiviruses . . . . . . . . . . . . . . . . . . . . . 31

Lentiviruses as research tools . . . . . . . . . . . . . 31

1.4 JC Polyoma virus derived virus-like particles . . . . . . . . . . . . . . 33

JC virus-like particles as delivery tool . . . . . . . . . . . . . . 34

1.4.1 Retargeting of virus-like particles . . . . . . . . . . . . . . 35

1.5 Aim of the thesis . . . . . . . . . . . . . . . . 36

2 Materials and Methods $\quad 37$

2.1 Materials . . . . . . . . . . . . . . . . . . 37

2.1.1 Chemicals and enzymes . . . . . . . . . . . . . 37

2.1.2 Oligonucleotides . . . . . . . . . . . . . . . . . . 40

2.1.3 Machines ......................... 42

2.2 Molecular biology . . . . . . . . . . . . . . . . . . . . 43

2.2.1 Enzymatic digestion of DNA . . . . . . . . . . . . . . . 43

2.2.2 Ligation of DNA . . . . . . . . . . . . . . . . . . . 43

2.2.3 Transformation of E.coli . . . . . . . . . . . . . . . . . . . . . . . . . . . . . . . 44

2.2.4 Purification of E.coli plasmids . . . . . . . . . . . . . . . . . . . 45 
2.2.5 Polymerase chain reaction . . . . . . . . . . . . . . 46

2.2.6 Quantitative real-time PCR . . . . . . . . . . . . . . . 47

2.2.7 Agarose gel electrophoresis . . . . . . . . . . . . . . . . . . . . . . . 48

2.2.8 DNA isolation from agarose gels and PCRs . . . . . . . . . . . . . . 49

2.2.9 MIDGE vector production . . . . . . . . . . . . . . . . 49

2.3 Cell biology . . . . . . . . . . . . . . . . . . . . . . 50

2.3.1 Cell culture . . . . . . . . . . . . . . . . . . 50 50

2.3.2 Primary material . . . . . . . . . . . . . . . 50

2.3.3 Primary cortical cultures . . . . . . . . . . . . . . . 51

2.3.4 Lipotransfection of cells . . . . . . . . . . . . . . . . . . . 52

2.3.5 rAAV production . . . . . . . . . . . . . 53

2.3.6 exo-AAV production . . . . . . . . . . . . . . 54

2.3.7 Reference rAAVs from the Viral Vector Core . . . . . . . . . . . . . 54

2.3.8 Iodixanol density gradient . . . . . . . . . . . . . . . 55

2.3.9 Analytical iodixanol gradient . . . . . . . . . . . . . 56

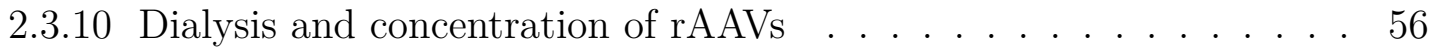

2.3.11 Lentivirus production . . . . . . . . . . . . . . 57

2.3.12 Flow cytometry analysis . . . . . . . . . . . . . 58

2.3.13 Determination of transducing units by flow cytometry . . . . . . . . 59

2.3.14 PEG precipitation of vesicles . . . . . . . . . . . . . . . . . 59

2.3.15 Nanoparticle tracking analysis . . . . . . . . . . . . . . . 59

2.3.16 VLP packaging and transduction . . . . . . . . . . . . . 59

2.3.17 VLP retargeting . . . . . . . . . . . . . . . . . . . . 60

2.3.18 Microscopy . . . . . . . . . . . . . . . . . 61

2.3.19 Voltage clamp recording . . . . . . . . . . . . . . . . . 61

2.4 Protein biochemistry . . . . . . . . . . . . . . . . . . . . 62

2.4.1 Protein expression in E.coli . . . . . . . . . . . . . . . . . . . . . . 62

2.4.2 Protein extraction . . . . . . . . . . . . . . . . . . . 62

2.4.3 Protein purification (FPLC) . . . . . . . . . . . 63

2.4.4 TCA protein precipitation . . . . . . . . . . . 63

2.4.5 SDS-PAGE, Coomassie and Western blot . . . . . . . . . . . . 63

2.4.6 Silver stain of SDS-PAGEs . . . . . . . . . . . . . . . . 66

2.5 Statistics and software . . . . . . . . . . . . . . 68

2.6 Cortex injection and tissue staining of Macaca mulatta . . . . . . . . . . 68

2.7 Cochlea injection and tissue staining of mice . . . . . . . . . . . . . . . . . 69 
3 Results $\quad 71$

3.1 Standard rAAV production and evaluation . . . . . . . . . . . . . . 71

3.1.1 Purification of standard rAAVs . . . . . . . . . . . . . . 73

Centrifugational purification yields a minor enrichment of rAAVs . . 73

Density gradient based purification efficiently enriches rAAVs . . . . 76

rAAV column purification . . . . . . . . . . . . 78

3.1.2 Quality control comparison with reference in vivo quality viruses . . 78

Virus titer and functionality . . . . . . . . . . . . . . . . . . 78

Silver stain for the visualisation of proteins . . . . . . . . . . . . 80

Electron microscopy for differentiation between full and empty capsids 81

Mass spectrometry identifies co-purified proteins . . . . . . . . . . . 84

Genetic toolbox for straightforward expression construct generation 85

Quick readout of opsin functionality in HeLa cells . . . . . . . . . . 87

Summary of rAAV production, purification and quality control . . . 88

3.1.3 Ex vivo and in vivo applications of $\mathrm{rAAVs} \ldots$. . . . . . . . . . . . 89

rAAVs transduce human MSCs and cortex tissue of murine and non-human primate origin . . . . . . . . . . . . . . 89

In vivo rAAV5 functionality . . . . . . . . . . . . . . . . . . . 93

rAAV5 transduces supporting cells in the cochlea of mice . . . . . . 93

rAAV5 expression in the cortex of Macaca mulatta . . . . . . . . . . 95

$3.2 \mathrm{CD}_{\mathrm{GFP}}$ over-expression for a higher yield of exo-AAV1 . . . . . . . . . . 97

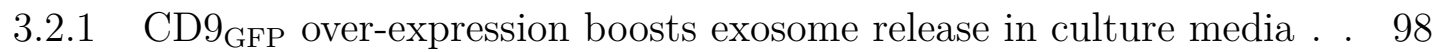

3.2.2 CD9 $9_{\text {GFP }}$ over-expression yields more efficient and increased amounts of exo-AAVs . . . . . . . . . . . . . . . . . . 100

3.2.3 Exo-AAV serotypes 1 and 6 transduce mesenchymal stem cells . . . 104

3.3 Lentiviruses as neuronal delivery tool . . . . . . . . . . . . . . . . 106

3.4 Virus-like particles as delivery tool for neuronal targets . . . . . . . . . . . 108

3.4.1 Delivery of linear expression cassettes and MIDGE vectors . . . . . 108

3.4.2 Virus-like particles for neuro-optogenetics . . . . . . . . . . . . 110

Neurotrophic retargeting of virus-like particles . . . . . . . . . . . . 111

4 Discussion

4.1 Standard rAAV production and evaluation . . . . . . . . . . . . . . 117

4.1.1 Standard rAAV production and evaluation on human cell lines . . . 117

4.1.2 Quality control and comparison with a reference virus . . . . . . . . 120 
4.1.3 Application of rAAVs ex vivo and in vivo . . . . . . . . . . . . . . . 124

Ex vivo application of rAAV serotypes to elucidate their potential

for gene therapy . . . . . . . . . . . . . . . . . 124

In vivo applications . . . . . . . . . . . . . . . . . . . . . . 126

$4.2 \mathrm{CD} 9_{\mathrm{GFP}}$ over-expression increases the yield of exo-AAV1 . . . . . . . 127

4.2.1 exo-AAV serotypes 1 and 6 reveal a different expression profile on mesenchymal stem cells than standard rAAVs . . . . . . . . . . . . 129

4.3 Lentiviruses as neuronal delivery tool . . . . . . . . . . . . . . . . . 130

4.4 Virus-like particles as delivery tool for neuronal targets . . . . . . . . . . . 131

4.4.1 Safe DNA delivery by combination of VLPs and MIDGE vectors . . 131

4.4.2 Neuronal expression and retargeting of VLPs . . . . . . . . . . . . 132

4.5 Conclusion and future directives . . . . . . . . . . . . . . . 135

Bibliography

5 Appendix $\quad 155$

5.1 Plasmid maps . . . . . . . . . . . . . . . . . . . . . 155

5.1 .1 rAAV genetic toolbox . . . . . . . . . . . . . . 155

5.1.2 Linear expression cassette plasmids . . . . . . . . . . . . . . . 159

$5.1 .3 \mathrm{scFv}$ expression plasmids . . . . . . . . . . . . . . 160

5.2 Mass spectrometry analysis of impurity lanes . . . . . . . . . . . . . . . 161

5.3 rAAV5 injection in the cochlea of mice . . . . . . . . . . . . . . . . 169

5.4 The tetraspanin CD9 enhances exo-AAV production . . . . . . . . . . . . 170

5.5 The tetraspanin CD9 enhances exosome secretion and lentivirus infectivity 172 


\section{List of Figures}

1 Application routes for gene therapy . . . . . . . . . . . . . . . . 20

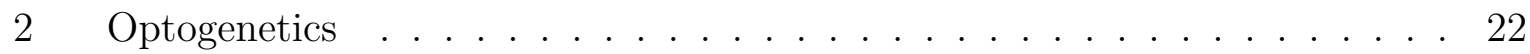

3 Adeno-associated virus . . . . . . . . . . . . . . . . . . . 24

4 Exosome-associated AAVs . . . . . . . . . . . . . . . . . . . . . . . 29

5 Structural organisation of VP1 VLPs . . . . . . . . . . . . . . . 35

6 Transduction of five human cell lines by five rAAV serotypes with three promotors . . . . . . . . . . . . . . . . . . . 72

7 Sequential centrifugation protocol leads to a minor rAAV enrichment . . . 75

8 rAAV2/5-CAG-GFP disribution in iodixanol density gradient: genomic and functional titer . . . . . . . . . . . . . . . . 76

9 Purity of concentrated gradient fractions . . . . . . . . . . . . . . . 77

10 Genomic and functional titer of reference and self-produced viruses . . . . 79

11 Silver stained SDS-PAGES for purity evaluation of rAAV samples . . . . . 81

12 Visualisation of rAAV capsids by electron microscopy . . . . . . . . . . . . 83

13 Schematic rAAV genetic toolbox . . . . . . . . . . . . . . 85

14 Illumination evoked response of light gated channelrhodopsin . . . . . . . . 88

15 Human MSCs are susceptible to five rAAV serotypes with different preference 89

16 Expression of rAAVs in dissociated Wistar rat cortex cultures . . . . . . . 91

17 Expression of rAAV5 constructs in acute cortex slices of Wistar rat and non-human primate origin . . . . . . . . . . . . . . . . . 92

18 Expression of rAAV5 in the mouse cochlea . . . . . . . . . . . . . . 95

19 Immunofluorescence stainings of rAAV5 injected Macaca mulatta cortex . . 96

20 Overview of HEK-AAV-CD9 $9_{\mathrm{GFP}}$ cell production and standard and exoAAV harvest . . . . . . . . . . . . . . . . . . . . . . . . . 98

$21 \mathrm{CD}_{\mathrm{GFP}}$ over-expression in HEK-AAV cells influences the vesicle content in the media and the samples during exo-AAV harvesting procedure . . . . 100

22 Enhanced transduction efficiency of exo-AAV1-CD9 $9_{\mathrm{GFP}} \ldots \ldots$. . . . . . . . 102

23 Exo-AAV1-CD9 $9_{\mathrm{GFP}}$ enriched in lower-density fractions . . . . . . . . . . 104

24 Exo-AAV and exo-AAV-CD9 ${ }_{\mathrm{GFP}}$ of serotypes 1 and 6 transduce mesenchymal stem cells . . . . . . . . . . . . . . . . . . . . 105

25 Lentiviruses deliver the transgene in HeLa cells . . . . . . . . . . . . . . . . . . . . . . . . . .

26 Production of MIDGE vectors . . . . . . . . . . . . . . . . . . . . 109

27 SKBR3 cells express linear DNA and MIDGE vector after VLP delivery . . 110

28 Production and retargeting strategy of VLPs . . . . . . . . . . . . . . 112

29 VLP retargeting to Her2/neu positive cells . . . . . . . . . . . . . . . . . . 113 
30 TrkBscFvs were expressed in E. coli but were only found in the unsoluble fraction . . . . . . . . . . . . . . . . . . . . 114

31 RVGN6 retargeting of VLPs . . . . . . . . . . . . . . . 116

32 Workflow of rAAV production, purification and quality control . . . . . . . 121

33 Conclusion figure . . . . . . . . . . . . . . . . . 135

34 GFP evaluation of rAAV5 expression in the mouse cochlea . . . . . . . . 169

35 Detection of exosomal markers, rAAVs, and GFP in vesicles of HEK-AAV and HEK-AAV-CD9 $9_{\mathrm{GFP}}$ cells . . . . . . . . . . . . . . 170

36 Transduction efficiency of media and 100k pellet of rAAV1 produced in HEK-AAV and HEK-AAV-CD9 $9_{\mathrm{GFP}}$ cells . . . . . . . . . . . . . . . . 171

37 The tetraspanin CD9 enhances exosome secretion and lentivirus infectivity 172 


\section{List of Tables}

2 Chemicals, media and enzymes . . . . . . . . . . . 37

3 Oligonucleotides quantitative real-time $\mathrm{PCR} \ldots \ldots \ldots \ldots$

4 Oligonucleotides scFv . . . . . . . . . . . . . . . . . . 40

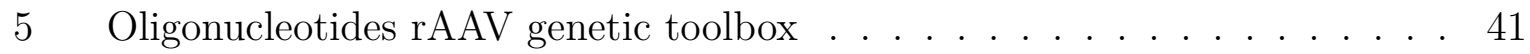

6 Oligonucleotides MIDGE . . . . . . . . . . . . . . . . . . . . . 41

7 Machines with model and manufacturer . . . . . . . . . . . . . . 42

8 LB medium . . . . . . . . . . . . . . . . . . . 44

$9 \quad$ SOC medium . . . . . . . . . . . . . . . . . . . . 44

10 Antibiotic concentrations in LB media . . . . . . . . . . . . . 45

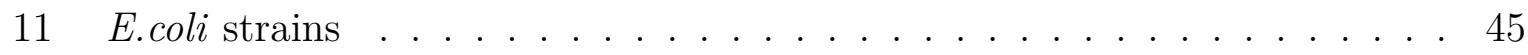

12 Real-time PCR program . . . . . . . . . . . . . . . . 48

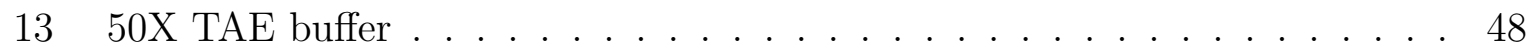

14 Cell lines and cultivation . . . . . . . . . . . . . . . 50

$15 \quad \mathrm{MSC}$ donors . . . . . . . . . . . . . . . . . . . . . . . 51

16 Dissection media . . . . . . . . . . . . . . . . . . 52

17 First-day media . . . . . . . . . . . . . . . . . . . . 52

18 Neurobasel media . . . . . . . . . . . . . . . . . . . . . . 52

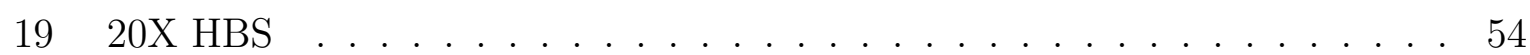

20 Standard rAAVs from the Viral Vector Core . . . . . . . . . . . . . 55

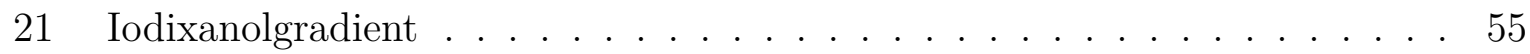

22 Iodixanol gradient solutions in $\mathrm{ml} \ldots \ldots \ldots \ldots \ldots$

23 10X Gradient buffer . . . . . . . . . . . . . . . . . . . 56

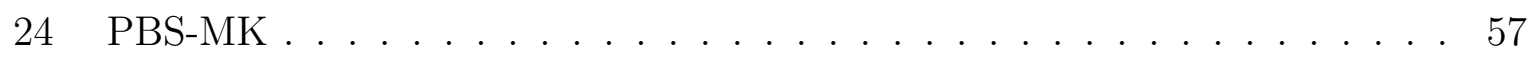

$254 \%$ Paraformaldehyde . . . . . . . . . . . . . . . . 58

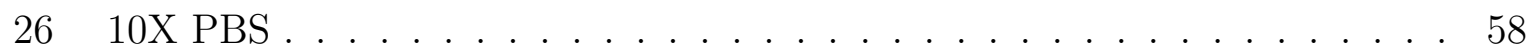

27 Reassociation buffer . . . . . . . . . . . . . . . 60

28 10X Dissociation buffer . . . . . . . . . . . . . . . . 60

29 Sorensen's buffer solution with PFA . . . . . . . . . . . . 61

30 5X Electrophorese buffer . . . . . . . . . . . . . . . . . . 64

$31 \quad 10 \%$ SDS-PAGE . . . . . . . . . . . . . . . . . . 64

32 Coomassie stain . . . . . . . . . . . . . . . . . 65

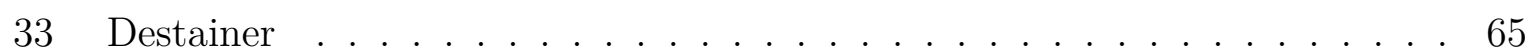

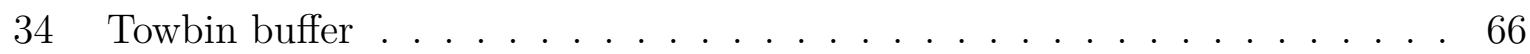

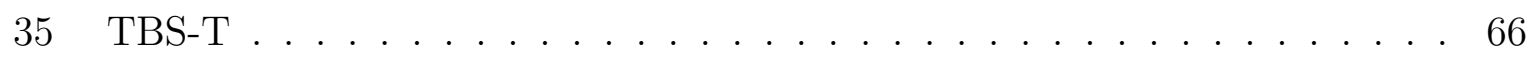

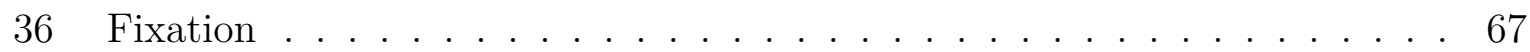


37 Reduction . . . . . . . . . . . . . . . . . 67

38 Silver stain . . . . . . . . . . . . . . . . . . . 67

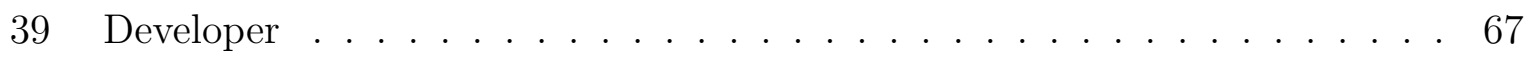

40 rAAV column purification . . . . . . . . . . . . . 78

41 Top ten mass spectrometry hits . . . . . . . . . . . . . . . . . . 84

42 rAAV plasmids from Addgene . . . . . . . . . . . . . . . 86

43 Modified rAAV plasmids . . . . . . . . . . . . . . . . . . 87

44 Ratio of vector genomes per transducing units for 100k pellet and media . 103

45 Mass spectrometry hits . . . . . . . . . . . . . . . . . . 161 


\section{Abbreviations}

aa

AAV

$\mathrm{ab}$

APS

Amp

$\mathrm{AU}$

b-ME

BSA

bp

$\mathrm{Ca}$

Da

$\mathrm{ddH}_{2} \mathrm{O}$

$\operatorname{div}$

DMEM

DMSO

DNA

dNTP

ds

E.coli

ECL

EDTA

exo-AAV amino acid

adeno associated virus

antibody

ammonium persulfate

ampicillin

arbitrary unit

beta mercaptoethanol

bovine serum albumin

baise pair

calcium

dalton

double distilled water

days in vitro

Dulbecco's Modified Eagle Medium

dimethylsulfoxide

desoxyribonucleic acid

desoxynucleotide triphosphate

double stranded

Escherichia coli

enhanced chemiluminescence

ethylendiamine tetraacetate

exosome-associated AAV 


\begin{tabular}{|c|c|}
\hline GFP & green fluorescent protein \\
\hline GOI & gene of interest \\
\hline $\mathrm{h}$ & hours \\
\hline HRP & horse radish peroxidase \\
\hline IHC & inner hair cell \\
\hline IPTG & isopropyl beta-D-1-thiogalactopyranoside \\
\hline $\mathrm{k}$ & kilo \\
\hline $\mathrm{kDa}$ & kilodalton \\
\hline MCS & multiple cloning site \\
\hline $\min$ & minutes \\
\hline $\mathrm{m}$ & milli \\
\hline $\mathrm{NeuN}$ & neuronal nuclear antigen \\
\hline$\mu$ & micro \\
\hline MIDGE & minimalistic, immunologically defined gene expression construct \\
\hline $\mathrm{Mg}$ & magnesium \\
\hline mRNA & messenger RNA \\
\hline MW & molecular weight \\
\hline NTA & Nanoparticle Tracking Analysis \\
\hline PAGE & polyacrylamide gel electrophoresis \\
\hline P.pastoris & Pichia pastoris \\
\hline PBS & phosphate buffered saline \\
\hline PCR & polymerase chain reaction \\
\hline PEG & polyethylene glycol \\
\hline PFA & paraformaldehyde \\
\hline
\end{tabular}




$\begin{array}{ll}\text { RNA } & \text { ribonucleic acid } \\ \text { rpm } & \text { revolutions per minute } \\ \text { S } & \text { seconds } \\ \text { sDS } & \text { sodium dodecyl sulphate } \\ \text { siRNA } & \text { small interfering RNA } \\ \text { ss } & \text { single stranded } \\ \text { TCA } & \text { trichloroacetic acid } \\ \text { Tris } & \text { tris(hydroxymethyl)aminomethane } \\ \text { U } & \text { units } \\ \text { VLP } & \text { Virus-like particle } \\ \text { wt } & \text { wild type } \\ \mathrm{Xg} & \text { x-fold gravity }\end{array}$





\section{Abstract}

Gene delivery tools play a key role in research and gene therapy. Since 1989, when the first trial in humans took place, the improvement of transduction efficiency and safety gained enormous attention to increase the therapy success.

In this context, the biotechnological aim of this thesis is to engineer three viral- and nonviral delivery tools.

I developed a production and purification pipeline for in vivo grade recombinant adenoassociated viruses (rAAVs) and established in vitro and ex vivo quality tests. By examination of the expression profiles of five rAAV serotypes on human cell lines and primary cells of human, rodent and non-human primate origin, I can additionally provide a source which enables the selection of the best-performing serotype for future applications. Additionally, I generated a genetic toolbox for a straightforward expression construct generation, useful for all three provided delivery tools.

Exosomes cannot only be used as biomarkers of diseases or as delivery tools for therapy, but in combination with rAAVs, they are a powerful tool to enhance the AAVs ${ }^{6}$ transduction efficiency, and immunological safety. Here, we used the expertise in boosting the exosome release by over-expression of the tetraspanin CD9, an exosomal marker-protein, to engineer the production of exosome-associated AAVs (exo-AAVs), the second part of my thesis. For the first time, we demonstrated that with this modification of producer cells, exo-AAV production yields up to $32 \%$ more efficient viruses.

Lentiviruses, another well-established vector system in gene therapy, were introduced and investigated to complete our gene delivery platform. Which is useful to unravel the mechanism behind the CD9's boosting effect.

The third tool is represented by JC polyoma virus-derived virus-like particles (VLPs). This quasi non-viral system is a promising tool for both research and clinical applications. In order to increase the immunological safety of linear DNA delivery with the VLPs, we adopted and investigated the utilisation of so-called MIDGE vectors, a monomolecular linear DNA cassette, as cargo.

By attachment of targeting molecules to the outer surface of the VLPs, we could alter the VLPs ${ }^{6}$ tropism towards new target cells. Using this strategy, I aimed to develop a retargeting towards nervous tissue which can by interchanging the targeting molecule also be applied for lentiviruses and exo-AAVs.

In summary, these developments provide a handy way to improve the AAVs $^{6}$ and exo$\mathrm{AAVs}^{6}$ production, purification and quality assessment. In addition, we provide a platform 
for viral (rAAV, Lentivirus) and non-viral (VLP) gene delivery systems with interchangeable expression features and cell-/tissue targeting opportunities. 


\section{Introduction}

Since the first human trial in 1989, gene therapy has emerged as a revolutionary possibility to treat or even cure genetic diseases and infectious disorders (Rosenberg et al., 1990). In the following, I will provide a short introduction to the field of my biotechnological thesis, with its scope to provide good delivery tools for gene therapy. For this purpose, I will firstly introduce the field of gene therapy in general and also exemplify its specific application in optogenetics.

Two viral vectors, recombinant adeno-associated viruses (rAAVs) and lentiviruses, will be described in more detail, including the rather shortly known exosome-associated AAVs, which represent the core tool in this thesis. Additionally, a virus-like particle technology and its options of a re-targeted tropism are presented.

\subsection{Gene therapy}

Gene therapy is the transfer of genetic material with the scope to modify specific gene functions of the receiving cell. Within this, three major modification routes can be classified: Gene augmentation for directed exogenous protein expression, gene correction and gene-silencing or activation (Kay, 2011).

All these approaches have the need of an efficient and specific gene-delivery to the target cells or tissues in common. The target can be reached by in vivo injection or ex vivo treatment of cell explants with subsequent allogeneic or autologous transplantation (Figure 1). The latter, for example, is successfully applied to hematopoietic stem cells (HSCs) which are isolated from the blood or bone marrow. After a viral treatment that modifies their gene expression, they are reposited by a systemic injection (Biffi et al., 2013; Aiuti et al., 2013). Back in the organism, the engineered HSCs can cross the blood-brain barrier and differentiate into microglia-like cells, expressing the therapeutic construct. These cells, for example, have been applied to treat X-linked adrenoleukodystrophy (X-ALD) that express sufficient amounts of the ALD protein after treatment (Aiuti et al., 2017; Cartier et al., 2009).

Another promising approach is the correction of frameshift mutations to restore gene functions. For example, transcription activator-like effector nucleases (TALENs) driven 
approaches restore the dystrophin mutation that causes Duchenne muscular dystrophy (Ousterout et al., 2013). RNA interference strategies showed in rodent models for Huntington's disease the succesful downregulation of the mutant huntingtin gene and thereby an inhibition of the disease causing protein aggregation (Franich et al., 2008; Stanek et al., 2014).

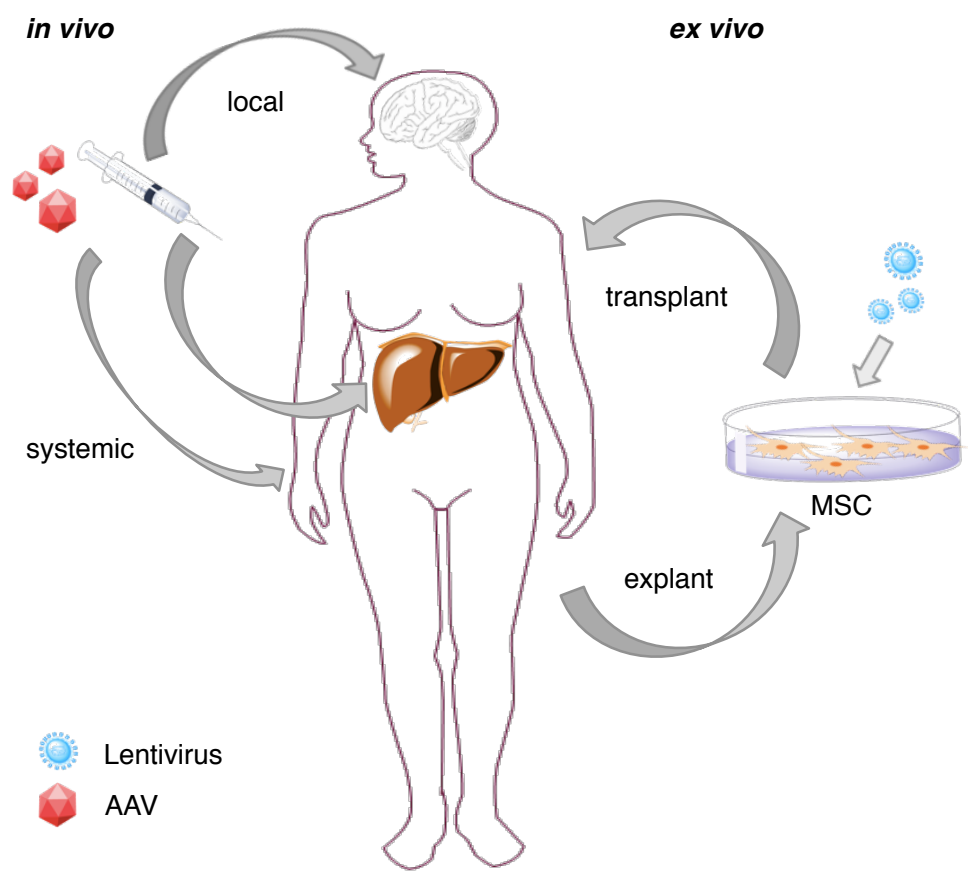

Figure 1: Application routes for gene therapy Delivery tools can be directly applied to the organism's target region or blood stream via injection. Alternatively, cell explants can be treated ex vivo and re-implanted afterwards.

Researchers and clinicians use several strategies for the delivery of nucleotides, including cationic liposome technologies or polycations, such as poly-l-lysine or polyethyleneimine (San et al., 1993; Boussif et al., 1995). These methods use the formation of nanoparticles incorporating the DNA or RNA. The cells are incubated with these complexes and incorporate them by the endosomal pathway. Another physical method is the electroporation, in which an electrical field increases the permeability of the cell membrane and the uptake of nucleotides. The latter is well-established for T-cell modification (Till et al., 2008; Zhao et al., 2006; Van Tendeloo et al., 2000).

However, these methods have limitations, such as not transfecting specific cell types or having a limited access to the brain. Furthermore, the targeted delivery to cell subpopulations is also more complicated to achieve. Circumventing these just named issues, 
biological tools like viruses have a big potential. They can even deliver the transgene through physical barriers like the blood-brain barrier or the inner limiting membrane of the retina (Foust et al., 2009; Duque et al., 2009; Dalkara et al., 2009).

\subsubsection{Viral vectors in gene therapy}

The application of viral vectors in gene therapy is constantly increasing (Chapin and Monahan, 2017). A variety of them are used including adenoviruses, herpes simplex viruses, lentiviruses, AAVs, and others (Crystal, 2014; Wold and Toth, 2013; Garrido et al., 1998; Tomás et al., 2013; Hocquemiller et al., 2016).

The first virus-based gene therapy which was available on the European market is Glybera ${ }^{\circledR}$, an AAV-based treatment of the rare disease lipoprotein lipase (LPL) deficiency. The lentivirus-based therapeutic called Stimvelis ${ }^{\circledR}$, used to treat patients with adenosine deaminase severe combined immunodeficiency (ADA-SCID), can also be found in clinics (Aiuti et al., 2017; Melchiorri et al., 2013).

Pre-clinical tests in large animal models are frequently under debate, as researchers are underlining the importance of these tests before proceeding with humans. Of course, this public debate is currently stressing the ethical concerns. Therefore, as far as possible, it is important to test potential cures in adequate in vitro and ex vivo approaches before testing them in living organisms.

But, however, in vitro and ex vivo test systems have the limitation that they just reconstruct a restricted part of the whole body and the in vivo efficiency and the required viral load can only be roughly estimated. Especially for systemic injections, one can only assume how the delivery tool will spread in the body and which off-targets will be transduced. Nevertheless, numerous models help to reduce animal experiments, man power and costs such as cerebral organoid models of the human brain, cultured human donor retinal explants, iPS-derived human retinal organoids, models for the blood-brain barrier or microfluidic organs-on-chips which are all therefore developed (Lancaster et al., 2013; Orlans et al., 2018; Quinn et al., 2018; Garberg et al., 2005; Cho et al., 2015; Helms et al., 2016; Bhatia and Ingber, 2014; Mewes et al., 2012). 


\subsubsection{Optogenetics in research and for therapy}

Optogenetics, as the name says, combines the genetic with an optic approach to modulate the electrical activity of excitable cells (Figure 2). Its history began in 2004, with Pan, Bodyden, and Deisseroth being able to control the neuronal activity of opsin expressing cells in vitro by the stimulation with light for the first time (Boyden et al., 2005).

The genetic compounds of optogenetics are opsins, light-sensitive transmembrane proteins. Under exposure to light of a certain wavelength they change their conformation which results in an ion flow. Depending on the charge change, the cell is either depolarized or hyperpolarized. An inward directed cation flow for example depolarizes the neuronal membrane and triggers an action potential. The most frequently used opsins in early days were Channelrhodpsin-2, Halorhodopsin and ArchT with a toolbox of new variations allowing a red-shifted action spectrum or step-function to prolong the excitable state nowadays (Nagel et al., 2003; Boyden et al., 2005; Han and Boyden, 2007; Han et al., 2011; Gunaydin et al., 2010; Packer et al., 2012).

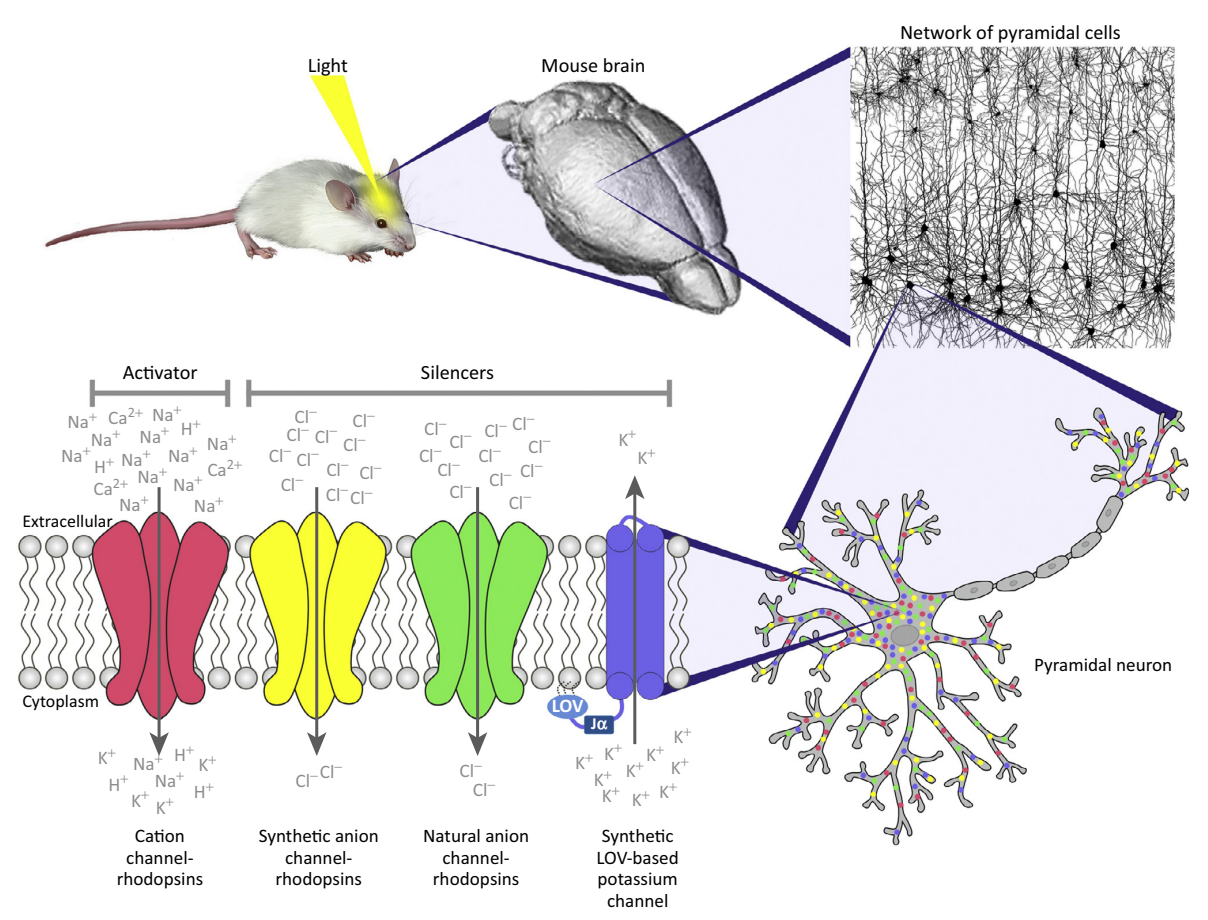

Figure 2: Optogenetics The light-controlled modulation of cell activity uses the expression of opsins. Depending on the induced ion-flow they act as activator or silencer. With permission from Kianianmomeni and Hallmann (2015).

Optogenetics is applied in various fields of research and potential clinical applications and is not any more limited to neurons, but also the light-driven modulation of glia cells and 
the control of the cardiac function comparable to a pacemaker are present applications (Pelluru et al., 2016; Arrenberg et al., 2010). Furthermore, the restoration of vision and hearing are under investigation (Bourzac, 2016; Moser, 2015). It might be a direct way to enable inner retinal cells to act as photoreceptors making them directly sensitive to light (Chaffiol et al., 2017; Kleinlogel, 2017). The optogenetic restoration of hearing is aiming at an improved frequency and intensity resolution, compared to conventional prosthetic cochlea implants for humans (Moser, 2015). Optogenetics finds its application in research to functionally and structurally map the connections of brain regions, or investigating the functions of sleep by the optogenetic modulation of the sleep behaviour in mice (Lim et al., 2013; Rolls et al., 2011). 


\subsection{Adeno-associated viruses}

Adeno-associated viruses (AAVs) have emerged as an important tool for research and clinics (Figure 3). They have been known since the 1960s when they were discovered as contaminants of adenovirus preparations, where they finally derived their name from (Atchison et al., 1965; Hoggan et al., 1966). They are about $25 \mathrm{~nm}$ small, non-enveloped viruses which bear a single-strand genome that can either be the minus- or the plus-strand (Hoggan et al., 1966; Berns and Rose, 1970). AAV is a member of the genus Dependoviruses, replication deficient and leads to a latent

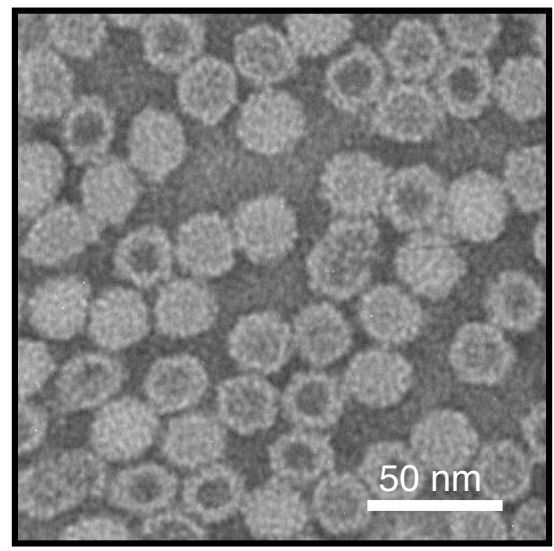

Figure 3: Adeno-associated virus Negative stained rAAV5 capsid in electron microscopy. infection with no connected disease.

For its replication it requires another virus that lyses the cell. This helper function can be provided by adenoviruses or herpesviruses (Hoggan et al., 1972). A dozen of serotypes and a hundred variants have been isolated, both differing in their genome and in capsid protein structure (Wu et al., 2006).

The AAV genome is flanked by two palindromic internal repeats (ITRs), which are required for genome replication and packaging. They are the only cis-acting elements in the viral vector. Consequently, the ITRs are the only viral sequences which are transferred to the recipient cells. The ITRs of AAV2 are usually used to flank recombinant expression constructs because they can be packed into capsids of different serotypes. Therefore, the nomenclature of a chimeric rAAV with ITRs of AAV2 in a serotype 5 capsid is rAAV2/5. However, to especially simplify labeling and when the result is based on the capsids, the ITR serotype is not declared but only the capsid serotype. Furthermore, AAV2 is the most studied wild-type virus, as mainly all molecular biology was undertaken with AAV2 in the first thirty years after its discovery and it was the first serotype to be developed into a recombinant vector for transgene delivery (Carter, 2004).

Three proteins, VP1, VP2, and VP3, form the capsid with 60 subunits in a 1:1:10 stoichiometry (Johnson et al., 1971). Each serotype has a characteristic tropism to specific cell types or tissues, that depends on the binding of capsid proteins to membrane glycosides. 
AAV5, for example, was described to use alpha-2,3-N-linked sialic acid with the plateletderived growth factor receptor as co-receptor (Walters et al., 2001; Kaludov et al., 2001). In contrast, AAV1 and AAV6 facilitate binding through $\alpha-2,3$ - and $\alpha$-2,6-N-linked sialic acid (Wu et al., 2006). A recent discovery identified a type 1 transmembrane protein mediating the cell entry of various serotypes. Now, this receptor is simply denoted as AAVR. It facilitates a rapid endocytosis and the trafficking to the trans-Golgi network (Pillay et al., 2016).

Additionally, in the case of in vivo injection, the AAVs tropism can vary depending on the applied concentration, making it even more challenging to find the best serotype for an application (Gerits et al., 2015; Taymans et al., 2007).

Whereas AAV2 is internalized via clathrin-coated pits (Bartlett et al., 2000), AAV5 enters the cell through clathrin-coated vesicles. Passing the endocytic pathway, they subsequently use a phospholipase domain in the capsid to escape the endosome. The capsid is then transported to the nucleus, the viral genome released and the host-cell DNA polymerase converts the single-stranded genome to a double stranded DNA (Fisher et al., 1996; Ferrari et al., 1996). But the trafficking of the various serotypes and the delivery of its DNA in the nucleus are still poorly understood.

Wild-type AAVs integrate within the site AAVS1 of the human chromosome 19 with a high precision (Samulski et al., 1991; Kotin et al., 1990). Thereby the rep protein mediates the site-specific integration. Integration occurs with a very low frequency using recombinant AAVs, because they usually lack the rep protein sequence in their genome (McCarty et al., 2004; Weitzman et al., 1994). The transferred expression construct mainly remains extrachromosomal as duplexed, circular episomes (Gerry et al., 1973). In non-dividing cells, the transgene expression can consequently persist for a sustained time, whereas it is transient in dividing cells.

Besides its gene delivery for extrachromosomal persistence, AAVs can also be used for gene-targeting. The single-stranded vector DNA serves as donor template for a homolog recombination to correct the host cell genome (Russell and Hirata, 1998). 


\section{Limitations of rAAVs for clinical approaches}

The use of rAAVs, especially for clinics, still faces some hurdles which are mainly caused by its:

- target specificity

- packaging limit

- good manufacturing process (GMP) production in high quantities

- immunology.

The following section will sum up these challenges including the current approaches to solve them.

Target specificity - Numerous studies have been undertaken to elucidate the viral tropism after systemic or local injection in small and large animal models (Gerits et al., 2015; Taymans et al., 2007; Zincarelli et al., 2008). The outcome can highly vary depending on the site of injection, the virus load, and the species. For example, numerous serotypes are used to target brain structures. These findings indicate the lack of a common knowledge about the most suitable serotype.

To tackle the hurdle of cell specificity, the serotypes are custom-designed via e.g. directed evolution of the capsid structure or conjugate-based targeting by the incorporation of high-affinity ligands into the latter (Wu et al., 2006; Yang et al., 2011; Ponnazhagan et al., 2002).

Regarding the expression construct, promoters are used which are specifically active in the target tissue or silenced in off-target tissues (Shevtsova et al., 2005; Geisler and Fechner, 2016). However, the constitutive CAG promotor is used in most expression constructs for clinical trials tackling CNS diseases such as Parkinson (Piguet et al., 2017). One approach which is mainly applied in small animal models is the use of transgene lines that stably express the cre-recombinase in specific tissues. After a widespread systemic rAAV application and transduction of various cells, the transgene expression is only activated in the cre-positive cells (Saunders et al., 2012).

Packaging limit - AAVs have a natural genome of approximately $4.7 \mathrm{~kb}$ in size, a limit also considered for a transgene capacity. Packaging of larger constructs lead to fragmented genome packaging and truncated protein expression (Wu et al., 2010). The so-called dual-AAVs are used to transfer the coding sequence for attempts where bigger proteins are required (McClements and MacLaren, 2017). For this, the formerly over-sized construct is split into two, partly overlapping sequences and a mixed population of the two 
rAAVs is applied. In cells which received both viruses, the gene recombines within the cell and facilitates the expression of the full-lengths protein. The full mechanism of how the recombination works is still unknown and it requires, of course, a highly efficient transduction of the cells with both viruses.

Furthermore, the double-strand synthesis is a rate-limiting step in transgene expression. Scientists developed the self-complementary rAAVs (scAAV) to achieve a faster onset of expression. The genome, flanked by ITRs, subsequently folds into a double-stand DNA that is covalently linked on one end (McCarty et al., 2003). Without the need of the second-strand synthesis in the cell, the expression of the gene can start earlier.

Production under GMP conditions - One of the biggest hurdles to make rAAV treatment available for a broad population is the GMP conform production in high amounts. New and modified production and purification protocols for rAAVs are constantly published nearly every month.

Depending on the serotype, AAVs mainly retain in the packaging cell line or are secreted into the media, leading to the need of different purification protocols (Vandenberghe et al., 2010). The standard harvesting procedures require cell lysis and virus separation from cell components and media by chromatographical methods or gradient centrifugation (Smith et al., 2003; Strobel et al., 2015; Zolotukhin et al., 1999). The purification needs to be adjusted to each serotype in particular and these facts demonstrate that not one gold standard protocol has been identified yet. Due to the inefficient and difficult process of scaling up rAAV production, it remains expensive, laborious and requires high experience to obtain a good quality, high titer virus.

The titer, the concentration of an AAV sample, can be given in three variants:

- the genomic titer of vector genome copie concentration $(\mathrm{vg} / \mathrm{ml})$ determined by dot blot or real-time PCR

- the capsid concentration determined by ELISA

- or the concentration of transducing units $(\mathrm{TU} / \mathrm{ml})$ that is determined by transduction assays.

Although the genomic titer is routinely used this can be problematic as it is comparably and reproducably determined in the same laboratory but can vary between laboratories. Additionally, depending on the purification method, the functionality of the viral preparation shows enormous variation. A preparation with $4 \times 10^{10} \mathrm{vg} / \mathrm{ml}$ and $75 \%$ uninfective particles for expample has the same infective potential as a a preparation with 
$2 \times 10^{10} \mathrm{vg} / \mathrm{ml}$ but $50 \%$ uninfective particles. The important difference is, that the latter comes with a lower load of capsids, which could trigger an immune response.

Clinical trials apply titers ranging from $10^{10} \mathrm{vg}$ in total to $10^{14} \mathrm{vg} / \mathrm{kg}$ body weight (Piguet et al., 2017). Normally the sample concentrations are $10^{13} \mathrm{vg} / \mathrm{ml}$, which then limits the possible virus load if the injection volume is also limited like for example in the brain.

Immunology - AAVs cause a latent infection with a mild immune response that can be cell-mediated or humoral (Mingozzi and High, 2013). Due to the lack of symptoms, the infection rate of the whole population is unknown and the subsequent abundance of neutralizing antibodies can only be diagnosed with an immunology test. The cytotoxic $\mathrm{T}$ cell-mediated response seems to be induced by the serotype and the route of application (Wang et al., 2007; Brockstedt et al., 1999).

The immune response is primarily humoral, mediated by neutralizing antibodies which were found in several preclinical animal models and human subjects with, to give an example, a prevalence of 59.5\% against AAV1 (Calcedo et al., 2015; Greenberg et al., 2016). Additionally, depending on the serotype and geographical area, the existence of antibodies vary. This factor dramatically reduces the transduction efficiency as well as the possibility of a second injection of the same serotype. To circumvent this hurdle both scientists and clinicians, check before the treatment whether the first choice serotype can be applied or whether an alternative one has to be administered. As well as for the serotype specificity, there is ongoing research for bioengineered capsids that will not be recognised by the antibodies (Paulk et al., 2017). Furthermore, exosome-associated AAVs are a variant of rAAVs, that overcome this hurdle, as described in the following section.

\subsubsection{Exosome-associated AAVs}

Numerous cell types of mammals, bacteria, plants and fungi release membraneous compartments from originally either intraluminal or plasma membranes into the surrounding milieu (Yoon et al., 2014; Blenkiron et al., 2016; Wang et al., 2013b; Joffe et al., 2016). These extracellular vesicles with 30-2,000 $\mathrm{nm}$ in diameter are classified depending on their size, with exosomes being 30-100 nm in diameter (Yoon et al., 2014; Théry et al., 2002). Additionally, they can be identified by some enriched proteins. These so-called exosomal marker proteins include the transmembrane tetraspanin CD9 (Hemler, 2005). Exosomes contain nucleic acids and proteins and act as message carriers between cells (Théry et al., 2002; Chiba et al., 2012; Cheng et al., 2014; Kalluri and LeBleu, 2016).

Within their first publication in 2012, Maquire et al. described a population of rAAVs 
that were associated with extracellular vesicles (Figure 4). These, later called exosomeassociated AAVs (exo-AAVs), have some functional advantages over the free rAAVs which will be described below (Maguire et al., 2012). Electron microscopy indicates that the rAAVs are located both inside the vesicle as well as attached to its surface (György et al., 2017; Fitzpatrick et al., 2014). Nevertheless, it still has to be elucidated how they enter or attach to the exosomes and whether this happens within or outside of the cell.

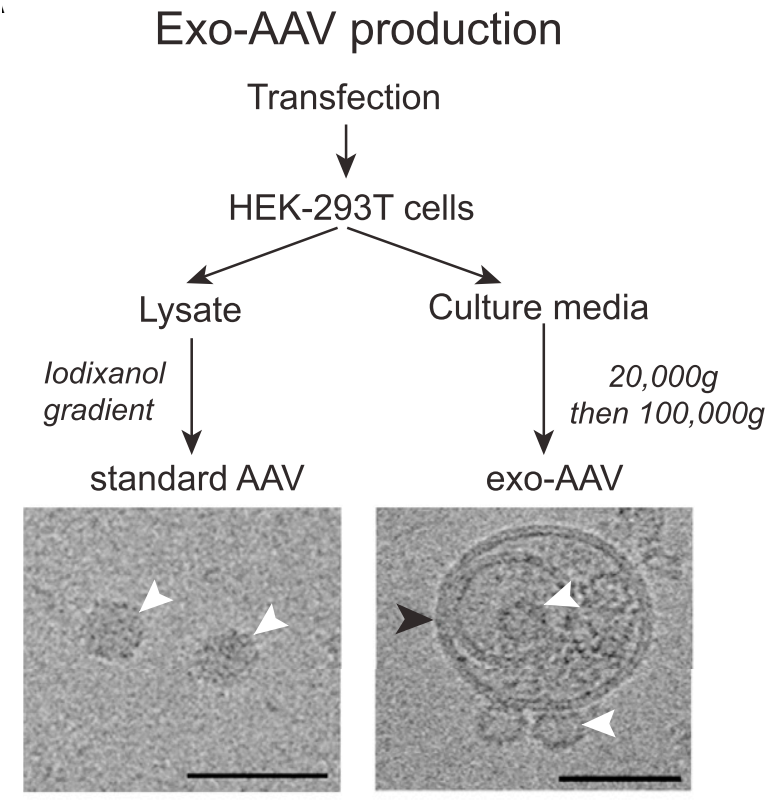

Figure 4: Exosome-associated AAVs Harvesting procedure of standard $\mathrm{rAAVs}$ and exo-AAV. Standard rAAV1 capsids are not associated with vesicles whereas this is clear for exo-AAVs. AAV capsids are marked with white arrowheads, whereas the lipid membrane is marked with the black arrowhead. Scale bar equals $50 \mathrm{~nm}$. Creative commons license CC BYNC-ND 4.0 (György et al., 2017).
Exo-AAVs have the potential to improve gene transfer via rAAVs. For example, exoAAVs of several serotypes are more efficient in transduction of cell culture and they presumably shield the rAAV vector against anti-AAV antibodies and increase its resistance (Maguire et al., 2012; György et al., 2014; Meliani et al., 2017). These beneficial features of exo-AAVs have also been proven by in vivo studies. ExoAAVs pass through the blood-brain barrier and the inner limiting membrane of the retina more efficiently compared to their free counterpart (Hudry et al., 2016; Wassmer et al., 2017). Moreover, exo-AAV9 and exo-AAV8 are more efficient in transduction of the CNS than their free rAAV counterpart (Hudry et al., 2016).

In contrast to free AAVs, exo-AAVs are harvested from the media by a sequential centrifugation with the final sample being recovered from the $100 \mathrm{k} \mathrm{x} \mathrm{g}$ pellet (Hudry et al., 2016). In this process, all cell-derived components which pellet in the $20 \mathrm{k} \mathrm{x}$ g centrifugation step are removed, as well as components that did not sediment at all. In contrast to the standard rAAV purification protocols aiming at a 
maximum pure sample, the exo-AAVs are rather impure, including undetermined supplements which might influence its transduction behavior as well. Similar to e.g. lentiviruses, exo-AAVs can be retargeted. For this purpose, the targeting-peptide like VSV-G is integrated in the lipid membrane of the exosome (Maguire et al., 2012; György et al., 2014). 


\subsection{Lentiviruses}

Lentiviruses are pathogens of animals and humans which cause a persistent infection with symptoms appearing after several months or years (Campbell and Robinson, 1998).

Lentiviral vectors for research and gene therapy are usually derived from the equine infectious anemia virus (EIAV), the siam (SIV), the feline (FIV), or the human immunodeficiency virus (HIV-1) (Wong et al., 2004; Kobayashi et al., 2003; Saenz et al., 2012; Liu and Berkhout, 2014).

Lentiviruses are single-stranded RNA viruses and, in contrast to AAVs, obtain a hostderived double lipid membrane envelope that they acquire upon budding from the cell membrane (Adamson and Jones, 2004). The envelope contains specific cellular receptors for the binding and final fusion with the cell membrane. Thereby, the viral core is released into the cell, disintegrates and its RNA genome is reverse transcribed into double-stranded cDNA. An active transport guides the viral DNA in the nucleus where it integrates into the host genome with the help of the virus-derived integrase (Bukrinsky et al., 1993). Therefore, lentiviruses have the ability to transfect dividing and non-dividing cells like neurons and stably express the transgene (Naldini et al., 1996).

Its natural genome is flanked by two long terminal repeats (LTRs) and harbors the genes for regulatory and accessory proteins. Furthermore it contains the enzymatic and structural genes env, pol, and also gag which codes for the envelope glycoproteins that interact with the host cell membrane, replication enzymes and the viral core, respectively. The LTRs have embedded the polyadenylation signal, promotor, and enhancer sequences that are important for viral gene expression and virus integration (Buchschacher and WongStaal, 2000).

\section{Lentiviruses as research tools}

In this study, we produced replication deficient HIV-1-based lentiviruses of the second generation that uses the co-transfection of a three plasmid system (Gruber et al., 2000; Zufferey et al., 1998). This system improves the safety of the production and application by avoiding the generation of replicative viruses because the viral genes are distributed on different plasmids. The cells are transfected with a packaging plasmids which codes for $g a g, p o l$, and rev, a plasmid that codes for the envelope proteins and the transfer vector which contains the expression cassette with the transgene flanked by the LTRs (Kim et al., 1998).

The capsid assembles in the cell and internalises the reverse transcribed expression cassette. The envelope proteins are synthesized by the cell and embedded in its plasma membrane. Exiting the cell by budding from the plasma membrane, the viral envelope 
contains a mixture of its proteins. Finally, the virus can be harvested from the conditioned cell culture media and subsequently directly applied or further purified (Coleman et al., 2003).

It is possible to alter the viral tropism by pseudotyping, i.e. to exchange the viral envelope proteins with other viral glycoproteins as previously mentioned for the exo-AAVs. For example, VSV-G from the vesicular stomatitis virus can be used to broaden the tropism and also the rabies virus glycoprotein (RVG) can be integrated to shift the tropism to the acetylcholine receptor (Burns et al., 1993; Mochizuki et al., 1998; Lentz, 1990). Additionally, engineered proteins like single chain antibodies can be integrated and exposed on the envelope (Friedel et al., 2015).

Lentiviruses have an advantage and disadvantage at the same time, namely being able to integrate into the host cell chromosomes. On the one hand, the transgene is stably expressed as long as the promotor is not silenced and the transduced cells are alive. On the other hand, however, the integration can lead to gene damages and bears an oncogenic potential (Schlimgen et al., 2016). To avoid negative effects, researchers have developed non-integrative lentiviruses that have a mutated integrase. All the valuable features, including highly efficient transduction of almost every cell type and the ease of production are maintained, but the expression cassette remains in an episomal viral form (Naldini and Verma, 2000; Delenda, 2004; Banasik and McCray, 2010). Due to their effective transduction and long-term expression even in the in non-human primates' cortex and also because of their easy production in research laboratories, lentiviruses have become a powerful tool for neuro-optogentic experiments. But, compared to the more stable AAVs which lack a membraneous envelope, the lentiviruses' lifetime is rather short, due to their rather fragile envelope (Han, 2012).

The first human clinical trial with lentiviruses was performed in 2003 with the objective to treat HIV infection with an antisense RNA expression against its envelope glycoproteins (Levine et al., 2006). Since then, an increasing number of clinical trials with lentiviruses for a wide range of human diseases have been performed. Thereby, the virus is used in a direct injection or for treatment of hematopoietic stem cells, including treatments for Parkinson's disease, Wiscott-Aldrich syndrome and X-linked adrenoleukodystrophy (ALD) (Palfi et al., 2014; Aiuti et al., 2013; Cartier et al., 2009). 


\subsection{JC Polyoma virus derived virus-like particles}

Virus-like particles (VLP) are virus particles that only consist of the viral capsid proteins and lack any viral nucleic acids. Many viruses have been used to generate these non-infectious VLPs, as the human papillomavirus and the murine or human JC polyomavirus (Kirnbauer et al., 1992; Tegerstedt et al., 2005; Salunke et al., 1986).

The human JC Polyoma Virus (JCV) was first isolated and cultured from a patient with progressive multifocal leukoencephalopathy. The patient's name was John Cuningham, whose initials were used to name the virus (Padgett et al., 1971). JCV has a broad prevalence in the human population, with over $50 \%$ having a silent infection (Olsson et al., 2013). The latent infection can give rise to progressive multifocal leukoencephalopathy (PML) in immunosuppressed individuals, caused by the infection and later result in fatal lytic destruction of astrocytes and oligodendrocytes (Ferenczy et al., 2012).

The native virus genome is a $5.1 \mathrm{~kb}$ double-stranded circular DNA molecule that is associated with histone core proteins derived from the host cell in a chromatin-like complex (Frisque et al., 1984; Wollebo et al., 2013). It is encapsulated in a non-enveloped, icosahedral protein capsid of 40.5-44 nm in diameter, composed of the three structural proteins VP1, VP2, and VP3 (Eash et al., 2006; Frisque et al., 1984; Goldmann et al., 1999). VP1 is a $42 \mathrm{kDa}$ protein and is the main capsid component with about $80 \%$ (Gillock et al., 1997). It alone forms pentamers and is able to form the outer capsid with 72 of these sub-units linked by its flexible, C-terminal arm (Salunke et al., 1986; Ou et al., 1999). The $\mathrm{Ca}^{2+}$ binding domains within the pentamers and the disulphide bonds between the pentamers stabilise the VP1 interactions (Chen et al., 2001). A DNA binding domain on VP1, that is not sequence specific, effectuates the encapsulation of the viral DNA (Moreland et al., 1991; Ou et al., 1999).

JCVs natural tropism is defined through its binding domains on the VP1 capsid surface. For an infection, the virus firstly attaches to the exposed $\alpha$-2,6-linked sialic acid on a lactoseries tetrasaccharide c (LSTc) glycan and secondly to the serotonin receptors

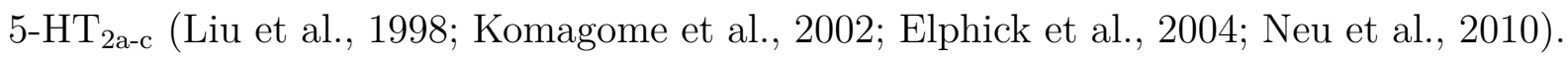
A ligand-inducible clathrin-dependent endocytosis leads to the cell-entry and subsequent trafficking to the ER with the initial uncoating of the virion (Pho et al., 2000; Querbes et al., 2004, 2006). Upon entry into the ER, they presumably use the ER-associated degradation machinery to translocate to the cytosol (Ferenczy et al., 2012). Low calcium concentrations in the cytosol destabilizes the virions and result in an exposure of nuclear localization signals at the N-terminus of VP1 and C-terminus of VP2 and VP3 that guide 
the transport into the cell's nucleus. The way towards the nucleus is guided via the intermediate filament network and the nucleus entry is facilitated by importins (Ashok and Atwood, 2003; Qu et al., 2004).

\section{JC virus-like particles as delivery tool}

The VLP technology is based on the recombinant expression of the VP1 protein of the human JC polyoma virus and this biotechnological tool thereby combines the advantages of a viral capsid and classical transfection methods to deliver foreign DNA or RNA.

Recombinant expression with self-organizing capsids has been demonstrated in, e.g. insect cells or E. coli (Chang et al., 1997; Ou et al., 1999).

More importantly, the JC VLPs have similar structural features and a similar tropism as their wild-type counterparts. Additionally, its biological characteristics regarding antigenicity and cellular infection are similar to the wild-type virions (Chang et al., 2011). Immunisation studies in which rabbits had an intravenous injection of purified VP1, have demonstrated that it did not induce an immune response, in contrast to an intramuscular injection together with an adjuvant (Goldmann et al., 1999). There is a promising study for the transfer to clinics in which JC VLPs were repeatedly administered in the rhesus macaque via three different application routes. There were not any signs of lasting activation of the cellular or humoral immune response (unpublished data).

For DNA delivery, the viral capsid can be loaded during the recombinant expression and co-transfection of the gene construct. Alternatively, under ambient buffer conditions, the loading procedure can be performed with the purified protein by osmotic shock or a disassembly/reassembly reaction in the presence of the DNA- or RNA-cargo. This feature enables the packaging of only the essentially desired sequences, such as siRNAs, linear DNA, PCR products or synthetic nucleic acids which in turn can even be delivered in hard-to-transfect cells like the human B-cell line Raji (Goldmann et al., 1999).

JC VLP was successfully applied in a rodent model system for osteoporosis and the delivery of siRNAs against the osteoporosis relevant target receptor activator for nuclear factor-kappaB ligand (RANKL). A silencing of around $30 \%$ RANKL expression was observed (Hoffmann et al., 2016). 


\subsubsection{Retargeting of virus-like particles}

Retargeting of delivery tools is the attempt to render and mask the native tropism by the attachment of exposed peptides on e.g. viral surfaces or exosomes. It is similarly possible for VLPs.

One of the VP1 exposed loops of the murine polyomavirus were modified by the integration of an antibody-binding domain (z-protein). This allowed the binding of antibodies and thereby to mask and alter the native tropism when used as VLPs (Gleiter and Lilie, 2001).

Utilizing this retargeting strategy for the JC VLPs, it was possible to direct the DNA delivery to human cancer cells by switching the JC VLPs tropism towards the human epidermal growth factor receptor $(2 \mathrm{Her} 2 / \mathrm{neu}$ ), that is particularly overexpressed in breast and colon cancers (see results 3.4.2). During the course of biochemical manufacturing, it is possible to attach nearly every targeting molecule, e.g. single chain antibodies ( $\mathrm{scFV}$ ), to lysin residues on the VLPs surface (Figure 5). Thereby the delivery to specific cell populations is raised, increasing its security for in vivo applications.

VP1 monomer

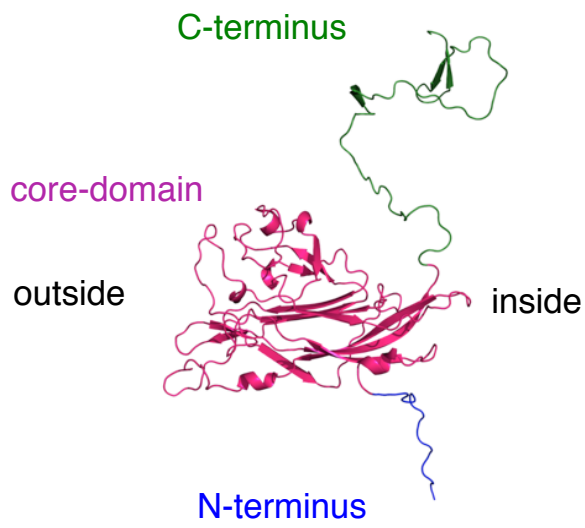

\section{VP1 pentamer}

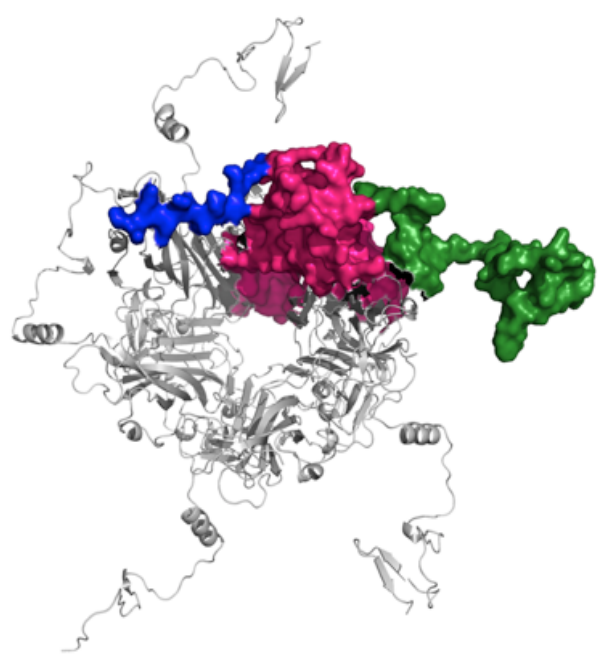

Figure 5: Structural organisation of VP1 VLPs The N-terminus of the VP1 protein stabilizes the pentamer and the C-terminus connects the adjacent pentamers which form the capsid. The outside of the core-domain exhibits lysin residues to which retargeting molecules are attached. PDB:15VA

Additionally, infections with JCV have been reported for a wide range of cell types, such as oligodendrocytes, astrocytes, glial cells, kidney cells, the bone marrow and B lymphocytes (White and Khalili, 2011; Ferenczy et al., 2012). More importantly and what still 
has to be taken into account when using the VLP as a delivery tool is that many studies equalise the infectivity of viruses for a cell type with its ability to enter the cell. But this can lead to confusions, as a natural virus might be able to enter the cell, not being expressionally active because its promotor is not active in this specific cell type (White et al., 2009). Now, using a VLP and a foreign expression cassette, the DNA is not only be delivered in the cell, but also expressed. This was shown for JC VLPs and, e.g. HeLa cells, in which the native virus enters but does not replicate (Suzuki et al., 2001; Qu et al., 2004).

Due to the unique features of in vitro cargo-loading and the chemical alterations of the exposed surface to address the delivery to specific cell populations, its biological safety can be increased and it is also a promising tool for gene therapy. VLPs do not contain any viral nucleic acids which is an additional advantage over other delivery systems like rAAVs and lentiviruses. Because of the non-viral gene transfer, there is no risk of recombination with wild-type viruses in host cells and no risk of activation of oncogenes (Goldmann et al., 1999).

\subsection{Aim of the thesis}

Gene transfer for research and clinics still requires more efficient gene delivery tools and robust production pipelines. Therefore, this thesis aims to provide a platform of three delivery tools for gene transfer to neuronal targets. Thereby we mainly addressed:

- A pipeline for rAAV production up to in vivo application.

- The straightforward generation of expression constructs with our genetic toolbox.

- An enhanced exo-AAV production by over-expression of the exosomal marker CD9.

- Production of lentiviruses for efficiency comparison.

- Safer linear DNA delivery with neurotrophic retargeted VLPs. 


\section{Materials and Methods}

\subsection{Materials}

\subsubsection{Chemicals and enzymes}

All solutions were prepared with water, purified into aqua bidest quality $\left(\mathrm{ddH}_{2} \mathrm{O}\right)$ via the arium ${ }^{\circledR p}$ pro ultrapure water system. Sterilisation of material and reagents was operated by autoclaving or by filtration through a $0.2 \mu \mathrm{m}$ pore size disposable filter.

The assembly of solutions is described in the corresponding context.

Table 2: Chemicals, media and enzymes

\begin{tabular}{|c|c|c|c|}
\hline Substance & Order No. & Manufacturer & \\
\hline agar-agar KobeI & 5210 & Roth, Germany & \\
\hline $\mathrm{AgNO}_{3}$ & 6207.1 & Roth, Germany & \\
\hline Acetic acid & 6755.1 & Roth, Germany & \\
\hline Ammonium persulfate (APS) & 9592.3 & Roth, Germany & \\
\hline Benzonase $250 \mathrm{U} / \mu \mathrm{l}$ & E1014-5kU & Sigma, Gemany & \\
\hline B27 supplement & 17504 & Gibco, Germany & \\
\hline $\mathrm{CaCl}_{2}$ & $\mathrm{HNO} 4$ & Roth, Germany & \\
\hline $\mathrm{C}_{2} \mathrm{H}_{3} \mathrm{NaO}_{2}$ (water free) & S- 8750 & Sigma, Germany & \\
\hline Coomassie Blue R250 & 3862.1 & Roth, Germany & \\
\hline DMEM & 31966021 & Gibco, Germany & \\
\hline DNaseI & A3778 & $\begin{array}{l}\text { PanReac AppliChem, } \\
\text { many }\end{array}$ & Ger- \\
\hline EGTA & E3889 & Sigma, Germany & \\
\hline
\end{tabular}


Formaldehyde

Ethanol p.a.

Ethidium bromide

FCS

Glucose

GlutaMAX

Glutaraldehyde

Glycine

HBSS

HEPES

Imidazole

Iodixanol

IPTG

Isopropanol

KCL

$\mathrm{KH}_{2} \mathrm{PO}_{2}$

Lipofectamin 2,000

Lysozyme

Methanol

$\mathrm{MgCl}_{2}$

$\mathrm{MgSO}_{4}$

$\mathrm{NaCl}$

$\mathrm{Na}_{2} \mathrm{CO}_{3}$

$\mathrm{Na}_{2} \mathrm{~S}_{2} \mathrm{O}_{3} * 5 \mathrm{H}_{2} \mathrm{O}$
0335.2

5054.1

2218.2

10500

6780

35050061

3778.1

G8898

14170

HN78

56750

D1556

CN08.2

CP41.3

HN02.3

P018.2

11668

A3711

P717.1

HN03.1

8283.2

3957

A135.1

P034.1
Roth, Germany

Roth, Germany

Roth, Germany

Gibco, Germany

Roth, Germany

Gibco, Germany

Roth, Germany

Sigma, Germany

Gibco, Germany

Roth, Germany

Sigma, Germany

Sigma, Germany

Roth, Germany

Roth, Germany

Roth, Germany

Roth, Germany

Invitrogen, Germany

AppliChem, Germany

Roth, Germany

Roth, Germany

Roth, Germany

Roth, Germany

Roth, Germany

Roth, Germany 


\begin{tabular}{|c|c|c|}
\hline $\mathrm{Na}_{2} \mathrm{HPO}_{4}$ & $\mathrm{~T} 876$ & Roth, Germany \\
\hline Neurobasalmedia & 21103 & Gibco, Germany \\
\hline OptiMEM & 31652 & Gibco, Germany \\
\hline PEI & P3143 & Sigma, Germany \\
\hline Penicillin-Streptomycin & P06-07100 & PAN-Biotech, Germany \\
\hline $\begin{array}{l}\text { Phusion High-Fidelity DNA } \\
\text { Polymerase }\end{array}$ & F530S & Thermo Fisher, Germany \\
\hline PMFS & 6367.1 & Roth, Germany \\
\hline Rotiphorese Gel $40(37,5: 1)$ & $\mathrm{T} 802.1$ & Roth, Germany \\
\hline SDS & CN30.3 & Roth, Germany \\
\hline TEMED & 2367.3 & Roth, Germany \\
\hline Taq DNA polymerase & GC-002-0250 & Biotherm, Germany \\
\hline Tris & 4088.3 & Roth, Germany \\
\hline Trypan Blue & CN76.1 & Roth, Germany \\
\hline trypsin/EDTA $(0.05 / 0.02 \%)$ & P10-023100 & PAN Biotech \\
\hline T7 DNA polymerase & EP0081 & Thermo Fisher, Germany \\
\hline Tween 20 & 9127.1 & Roth, Germany \\
\hline T4 DNA ligase & M0202S & NEB, England \\
\hline Taq polymerase & $3000010-02-000003$ & Ares Bioscence \\
\hline Uranyl acetate & E22400-2 & Science Service, Germany \\
\hline XerumFree & XF205-0020-S1 & TNCBio, Netherlands \\
\hline Yeast extract & 2363.2 & Roth, Germany \\
\hline
\end{tabular}




\subsubsection{Oligonucleotides}

Desalted purified oligonucleotides were purchased from Sigma-Aldrich, Germany. They were used in a concentration of $10 \mu \mathrm{M}$ diluted in $\mathrm{ddH}_{2} \mathrm{O}$.

Table 3: Oligonucleotides quantitative real-time PCR

\begin{tabular}{lll}
\hline MRB Number & Description & Sequence $\mathbf{5}^{\prime} \mathbf{- 3}$ \\
\hline 298 & WPRE rev & CCGAAGGGACGTAGCAGAAG \\
305 & WPRE for & CTTGCCCGCTGCTGGAC \\
\hline
\end{tabular}

Table 4: Oligonucleotides scFv

\begin{tabular}{lll}
\hline MRB Number & Description & Sequence $\mathbf{5}^{\prime} \mathbf{- 3}^{\prime}$ \\
\hline 501 & Her2neu Xho for & GGCTCGAGAAGAGAGAAGCT \\
503 & Linker Streptavidin for & GGTTCTGGTTCTATGGGCATCACCGGCACC \\
504 & Streptavidin XbaI rev & CCTCTAGAGGGCAACCAGAACCACCC \\
605 & TrkBfor & CCATGGAAGCTGAGGCTAAGTACTTG \\
606 & TrkBCrev & GGATCCTCAATGATGATGATGATGATG \\
\hline
\end{tabular}


Table 5: Oligonucleotides rAAV genetic toolbox

\begin{tabular}{|c|c|c|}
\hline MRB Number & Description & Sequence $5^{\prime}-3^{\prime}$ \\
\hline 230 & Arch-YFP Nde for & (Phos)CATATGAAGAGCAGGATCACCAGCG \\
\hline 231 & Arch-YFP Nde rev & (Phos)GGCGGCCGCCTTGCTC \\
\hline 253 & CaMKII for & GGACCTGGATGCTGACGAAG \\
\hline 262 & $\begin{array}{l}\text { eNpHR-eYFP NdeI in } \\
\text { for }\end{array}$ & (Phos)CATATGGGCCGCCAAGAGCAGGATC \\
\hline 263 & $\begin{array}{l}\text { eNpHR-eYFP NdeI in } \\
\text { rev }\end{array}$ & (Phos)GCATCATCAGCCGGGGTCC \\
\hline 264 & $\begin{array}{l}\text { hChR- } \\
\text { mChe/tdTo/GFP } \\
\text { NdeI in for }\end{array}$ & (Phos)CATATGGTGAGCAAGGGCGAGGAG \\
\hline 265 & $\begin{array}{l}\text { hChR-mChe NdeI in } \\
\text { rev }\end{array}$ & (Phos)CATGGTGGCGGCCGCTGG \\
\hline 266 & ArchT-GFP NdeI in rev & (Phos)TGCTACTACCGGTCGGGGC \\
\hline 298 & WPRE rev & CCGAAGGGACGTAGCAGAAG \\
\hline
\end{tabular}

Table 6: Oligonucleotides MIDGE

\begin{tabular}{lll}
\hline MRB Number & Description & Sequence $\mathbf{5}^{\prime} \mathbf{- 3}^{\prime}$ \\
\hline 213 & rev CAG-GFP & TCTCCCCCTGAACCTGA AAC \\
214 & for pAAV-CAG & GATCGTACCATTGACGTCAATAATG \\
308 & WPRE out for & (Phos)TCGATACCGTCGACCCG \\
309 & WPRE out rev & (Phos)TTATCGATAAGCTTGATATCGAATTC \\
462 & MIDGE 1 & (Phos)CGCGTCTTTTGACGCGCATG \\
463 & MIDGE 2 & (Phos)CGCGTCTTTTGACGCGAGCT \\
\hline
\end{tabular}




\subsubsection{Machines}

Table 7: Machines with model and manufacturer

\begin{tabular}{|c|c|c|}
\hline Machine & Model & Manufacturer \\
\hline Balance & EG $620-3 N M$ \& Kern & Sohn GmbH, Germany \\
\hline Bioruptor & Bioruptor® Pico & Diagenode, Belgium \\
\hline Chemiluminescence system & ChemoCam Imager & INTAS, Germany \\
\hline Centrifuge & Heraeus Megafuge 8R & Thermo Fisher Scientific, USA \\
\hline Centrifuge & Heraeus Fresco21 & Thermo Fisher Scientific, USA \\
\hline Ultracentrifuge & Avanti J-30I & Beckman Coulter, USA \\
\hline Fluorometer & Qubit 2.0 & Life Technologies, USA \\
\hline Freezer $\left(-20^{\circ} \mathrm{C}\right)$ & & Bosch, Germany \\
\hline Freezer $\left(-80^{\circ} \mathrm{C}\right)$ & MDF-DU500VH-PE & Panasonic, Japan \\
\hline Freezer $\left(-150^{\circ} \mathrm{C}\right)$ & ULT7150-9-D & Thermo Fisher Scientific, USA \\
\hline Flow cytometer & LSR II Flow Cytometer & BD Bioscience, USA \\
\hline Gel documentation system & Gel iX Imager & INTAS, Germany \\
\hline Ice machine & ZBE $70-35$ & Ziegra, Germany \\
\hline Cell culture incubator & Heracell VIOS 160i & Thermo Fisher Scientific, USA \\
\hline Plate incubator & IN75 & Memmert, Germany \\
\hline Microscope & Axio Vert. A1 & Zeiss, Germany \\
\hline PCR thermocycler & Labcycler & Sensoquest, Germany \\
\hline $\mathrm{pH}$ meter & SevenCompactTM S210 & Mettler Toledo, USA \\
\hline Plate reader & Synergy 2 & BioTek, USA \\
\hline Platform rocker & PMR-30 & Grant bio, UK \\
\hline
\end{tabular}




\begin{tabular}{|c|c|c|}
\hline Power supply & Beckman & Consort bvba, Belgium \\
\hline Real-Time PCR System & 7500 Real-Time PCR System & Applied Biosystems, USA \\
\hline Real-Time PCR System & $\begin{array}{l}\text { StepOne }{ }^{\mathrm{TM}} \text { Plus } \\
\text { PCR System }\end{array}$ & Applied Biosystems, USA \\
\hline Rotor & JS-24.38 & Beckman Coulter, USA \\
\hline Rotor & JA-30.50 & Beckman Coulter, USA \\
\hline Safety cabinet & Safe 2020 & Thermo Fisher Scientific, USA \\
\hline Shaking incubator & Ecotron & Infors HT, Switzerland \\
\hline Sonifier & Sonifier 250 & BRANSON, USA \\
\hline Stereo microscope & SZ51 & Olympus, Germany \\
\hline Thermoblock & ThermoStat plus & Eppendorf, Germany \\
\hline Tissue slicer & 51425 & Stoelting, Ireland \\
\hline Ultrapure water system & arium ${ }^{\circledR}$ pro & Sartorius, Germany \\
\hline Vortexer & Vortex-GenieTM 2 & Scientific Industries, USA \\
\hline Water bath & WNB10 & Memmert, Germany \\
\hline Western blot chamber & XCell SureLock® MiniCell & Life Technologies, USA \\
\hline
\end{tabular}

\subsection{Molecular biology}

\subsubsection{Enzymatic digestion of DNA}

For an enzymatic digestion of DNA in order to create new constructs or confirm the composition of a plasmid the manufacturer's protocol was followed. Digestion samples were incubated between $30 \mathrm{~min}$ and $4 \mathrm{~h}$ at $37^{\circ} \mathrm{C}$. The gained DNA fragments were separated by agarose gel electrophoresis (2.2.7) and further purified as described in 2.2.8.

\subsubsection{Ligation of DNA}

For the creation of a new plasmid around $300 \mathrm{ng}$ insert DNA and around $10 \mathrm{ng}$ of the larger plasmid backbone DNA were mixed with $5 \mu \mathrm{l}$ reaction buffer and $1 \mu \mathrm{l}$ of T4 DNA 
ligase. It was filled up to a total volume of $50 \mu \mathrm{l}$ and incubated over night at $4^{\circ} \mathrm{C}$ or for half an hour at RT.

The ligation mixture was immediately used for transformation or stored at $-20^{\circ} \mathrm{C}$ until use.

\subsubsection{Transformation of E.coli}

Competent E.coli strains for plasmid transformations were prepared with the Mix \& Go E.coli Transformation Kit (Zymo Research, USA) according to the manufacturer's instructions. Aliquots of the competent cells were stored at $-20^{\circ} \mathrm{C}$ and thawed on ice before use. A few $\mu \mathrm{l}$ of a ligation mixture or plasmid preparation were incubated with the cells for 2 min on ice. Using plasmids coding for an Amp resistance the transformation mixture was immediately spread on an LB-plate containing Amp. In the case of other resistances, the transformation mixture was previously incubated for $1 \mathrm{~h}$ at $37^{\circ} \mathrm{C}$, at $300 \mathrm{rpm}$ in four volumes of SOC medium. Colonies grew over night at $37^{\circ} \mathrm{C}$.

Table 8: LB medium

\begin{tabular}{ll}
\hline LB medium & \\
\hline $\mathrm{NaCl}$ & $10 \mathrm{~g}$ \\
tryptone & $10 \mathrm{~g}$ \\
yeast extract & $5 \mathrm{~g}$ \\
\hline
\end{tabular}

Fill up to $1 \mathrm{~L}$ with $\mathrm{ddH}_{2} \mathrm{O}$ and sterile autoclave. $15 \mathrm{~g}$ of agar-agar KobeI was added for agar plates before autoclaving.

Table 9: SOC medium

\begin{tabular}{ll}
\hline SOC medium $\mathbf{1} \mathbf{L}$ & \\
\hline $\mathrm{NaCl}$ & $10 \mathrm{mM}$ \\
tryptone & $20 \mathrm{~g}$ \\
$\mathrm{MgCl}_{2}$ & $10 \mathrm{mM}$ \\
$\mathrm{MgSO}_{4}$ & $10 \mathrm{mM}$ \\
\hline
\end{tabular}

Add $20 \mathrm{mM}$ glucose after autoclaving. 
Table 10: Antibiotic concentrations in LB media

\begin{tabular}{ll}
\hline Antibiotic & Concentration \\
\hline Ampicillin & $100 \mu \mathrm{g} / \mathrm{ml}$ \\
Kanamycin & $50 \mu \mathrm{g} / \mathrm{ml}$ \\
Zeocin $^{\mathrm{TM}}$ & $100 \mu \mathrm{g} / \mathrm{ml}$ \\
\hline
\end{tabular}

Table 11: E.coli strains

\begin{tabular}{|c|c|c|}
\hline Strain & Genotype & Source \\
\hline $\begin{array}{l}\text { One } \text { Shot }^{\circledR} \text { Stbl } 3^{\mathrm{TM}} \text { Chemi- } \\
\text { cally competent cells }\end{array}$ & $\begin{array}{l}\text { F-mcrB mrrhsdS20(rB-, mB-) } \\
\text { recA13 supE44 ara-14 galK2 } \\
\text { lacY1 proA2 rpsL20 (StrR) } \\
\text { xyl-5 } \lambda \text {-leumtl-1 }\end{array}$ & Thermo Fisher Scientific, USA \\
\hline $\begin{array}{l}\text { One Shot }{ }^{\circledR T O P} 10 \text { Chemically } \\
\text { ompetent E.coli }\end{array}$ & 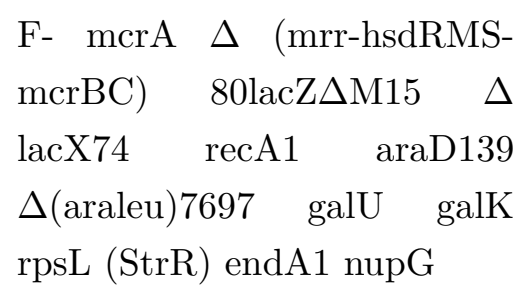 & Thermo Fisher Scientific, USA \\
\hline SoluBL21 ${ }^{\circledR}$ & $\begin{array}{l}\text { F- ompT hsdSB (rB- mB- ) gal } \\
\text { dcm (DE3) } \dagger\end{array}$ & Invitrogen, Germany \\
\hline BL21 Star ${ }^{\circledR}(\mathrm{DE} 3)$ & $\begin{array}{l}\text { F- ompT hsdSB (rB-mB-) gal } \\
\text { dcm rne131 (DE3) }\end{array}$ & Invitrogen, Germany \\
\hline
\end{tabular}

\subsubsection{Purification of E.coli plasmids}

Large scale preparation of E.coli plasmids was performed with the ZymoPURE ${ }^{\mathrm{TM}}$ Plasmid Midiprep Kit (Zymo Research, Germany) and 50-100 ml over night bacterial culture per column, according to the manufacturer's protocol.

For a small scale plasmid purification a $5 \mathrm{ml}$ over night culture of the bacterial strain was inoculated. $1.9 \mathrm{ml}$ culture was centrifuged at 21,000X g for $2 \mathrm{~min}$.

The pellet was resuspended in $150 \mu \mathrm{l}$ P1, and the same volume of P2 was added. The tubes were inverted several times and incubated for 5 min at RT before $150 \mu \mathrm{l}$ P3 was 
added and the tubes were inverted again. The cell debris was pelleted and the supernatant mixed with $500 \mu$ l isopropanol.

A centrifugation with $21,000 \mathrm{X}$ g for 20 min at $4^{\circ} \mathrm{C}$ pelleted the DNA, which was washed with $500 \mu \mathrm{l} 75 \%$ ethanol subsequently. The dried DNA pellet was resuspended in $100 \mu \mathrm{l}$ $\mathrm{ddH}_{2} \mathrm{O}$.

\begin{tabular}{ll}
\hline $\mathbf{P 1}$ & \\
\hline tris pH 8 & $10 \mathrm{mM}$ \\
EDTA pH 8 & $10 \mu \mathrm{g}$ \\
RNase A & \\
\hline & \\
\hline $\mathbf{P 2 a}$ & $400 \mathrm{mM}$ \\
\hline $\mathrm{NaOH}$ & \\
\hline $\mathbf{P 2 b}$ & $2 \%$ \\
\hline SDS
\end{tabular}

Mix P2a and P2b before use.

\begin{tabular}{ll} 
P3 & \\
\hline KAc & $3 \mathrm{M}$
\end{tabular}

Fill up with $\mathrm{ddH}_{2} \mathrm{O}$ and adjust $\mathrm{pH}$ to 4.8 with acetic acid.

\subsubsection{Polymerase chain reaction}

Polymerase chain reaction (PCR) is a method to amplify DNA sequences in vitro in a three step reaction. First, the dsDNA is denaturated at $95^{\circ} \mathrm{C}$, second at a lower temperature the primers anneal to the single strand DNA template, after that third the DNA polymerase elongates the primers to the complementary strand. With multiple repeats of that cycle, the region between the two primers is exponentially amplified. 
Here, the Taq polymerase and Phusion polymerase were used according to the manufacturer's protocol with primers listed in table 3.

A 5' phosphorylated primer pair, of which one primer had an overhang of the restriction side nucleotide sequence, was used to insert restriction sites in plasmids. The purified PCR product was recircularized with a standard ligation procedure and hereupon transformed in E.coli for further amplification.

\subsubsection{Quantitative real-time PCR}

Quantitative real-time PCR was used according to Rohr et al. (2002) to determine the concentration of AAV vector genomes (the genomic titer). Therefore the SensiMix ${ }^{\mathrm{TM}}$ SYBR ${ }^{\circledR}$ Low-ROX Kit and SensiMix ${ }^{\text {TM }}$ SYBR® Hi-ROX Kit (Bioline, UK) was used to set up a master mix with primers \#298 (5'-CCGAAGGGACGTAGCAGAAG-3') and \#305 (5'-CTTGCCCGCTGCTGGAC-3') according to the manufacturer's protocol. $1 \mu \mathrm{l}$ sample was added to $19 \mu \mathrm{l}$ master mix per 96-well plate well. The PCR run itself was performed with 7500 Real-Time PCR System or StepOne ${ }^{\text {TM }}$ Plus Real-Time PCR System and evaluated with 7500 System SDS Software, respectively StepOne ${ }^{\text {TM }}$ Software.

A ten-time dilution series ranging from $10^{3}$ templates $/ \mu$ l to $10^{8}$ templates/ $\mu$ l dsDNA was measured on the same plate to create a standard row according to which the concentration of viral vector genomes was calculated. Taking into account that the AAV genome is a ssDNA and to gain vector genomes per ml the value was doubled and multiplied by a factor of 1,000 .

The 4,464 bp long template DNA fragment was obtained by an enzymatic restriction digest of the plasmid pAAV-CaMKII-eNpHR3.0-eYFP with the enzyme SdaI, followed by a separation of the backbone by agarose gel electrophoresis and purification as described in 2.2.8. Its concentration was measured by Qubit 2.0 and aliquots of the standard dilution row were stored at $-80^{\circ} \mathrm{C}$. 
Table 12: Real-time PCR program

\begin{tabular}{lll}
\hline Duration & Temperature $\left({ }^{\circ} \mathbf{C}\right)$ & Step \\
\hline $10 \mathrm{~min}$ & 95 & holding stage \\
\hline $15 \mathrm{sec}$ & 95 & 40X cycling stage \\
$1 \mathrm{~min}$ & 60 & melting curve \\
\hline $15 \mathrm{sec}$ & 95 & \\
$1 \mathrm{~min}$ & 60 & \\
$15 \mathrm{sec}$ & +0.3 & \\
\hline
\end{tabular}

\subsubsection{Agarose gel electrophoresis}

Two-dimensional agarose gel electrophoresis is a method to separate DNA fragments according to their size. The gel consisted of $1 \mathrm{X}$ TAE buffer with $1 \%$ or $2 \%$ agarose. The samples were prepared with 6X loading dye (Thermo Fisher Scientific, Germany) and separated on an agarose gel using a field strength of $120 \mathrm{~V}$. A DNA marker (GeneRuler $1 \mathrm{~kb}$ DNA Ladder, Thermo Fisher Scientific, Germany) was used to determine the fragment size. Visualization of nucleotide fragments was performed after staining in an ethidium bromide bath $\left(1: 1000\right.$ in $\left.\mathrm{ddH}_{2} \mathrm{O}\right)$ with a gel documentation system.

Table 13: 50X TAE buffer

\begin{tabular}{ll}
\hline Substance & Concentration \\
\hline Tris base & $2 \mathrm{M}$ \\
EDTA & $50 \mathrm{mM}$ \\
acetic acid & $1 \mathrm{M}$ \\
\hline
\end{tabular}

Fill up with $\mathrm{dd}_{2} \mathrm{O}$ and adjust $\mathrm{pH}$ to 8.0. 


\subsubsection{DNA isolation from agarose gels and PCRs}

DNA in agarose gels was purified with QIAquick Gel Extraction Kit and DNA from PCR reactions was purified with QIAquick PCR Purification Kit (Qiagen, Germany) according to the manufacturer's protocol.

\subsubsection{MIDGE vector production}

MIDGE vector production was performed according to Schakowski et al. (2001). The CAG-GFP expression construct was amplified from the pAAV-CAG-GFP plasmid (Addgene \#28014) (for \#214, rev \#213). The amplicon was transferred via TA-cloning into the pGEM®-T Easy vector (Promega, Germany) to generate pGEM-T easy-CAGGFP-WPRE. Woodchuck hepatitis virus posttranslational regulatory element (WPRE) sequence was removed by PCR-amplification of the plasmid, followed by self-ligation (for \#308, rev \#309). For the MIDGE vector generation, the pGEM-CAG-GFP-WPRE was linearized with SacI and SphI followed by purification of the $1.9 \mathrm{~kb}$ DNA fragment as described in 2.2.8.

$2 \mu \mathrm{g}$ DNA was ligated over night at $16^{\circ} \mathrm{C}$ with $8 \mu \mathrm{l} 4$ Ligase and each $6.4 \mu \mathrm{l}$ of $100 \mu \mathrm{M}$ \#462 and \#463. DNA was purified using QIAquick PCR Purification Kit (Qiagen, Germany) before unligated oligonucleotides as well as not re-linearised DNA was digested with T7 DNA Polymerase. Therefore $1.5 \mu \mathrm{g}$ DNA was incubated with $60 \mathrm{U}$ T7 DNA Polymerase in a volume of $300 \mu \mathrm{l}$ for $6 \mathrm{~h}$ at $37^{\circ} \mathrm{C}$ and the reaction was stopped with an incubation of $20 \mathrm{~min}$ at $75^{\circ} \mathrm{C}$. The MIDGE vectors were purified using the QIAquick PCR Purification Kit. 


\subsection{Cell biology}

\subsubsection{Cell culture}

Cells were splitted twice a week and cultivated at $37^{\circ} \mathrm{C}$ with $5 \% \mathrm{CO}_{2}$ in a humidified atmosphere in T-75 or T-175 cell culture flasks (Sarstedt, Germany). The media was supplemented with penicillin/streptomycin (100 Units $/ \mathrm{ml}, 0.1 \mathrm{mg} / \mathrm{ml}$ ) and additional supplements listed in table 14 .

Table 14: Cell lines and cultivation

\begin{tabular}{|c|c|c|c|c|}
\hline Cell line & Tissue & Source & Media & FCS $(\%)$ \\
\hline HEK-293 & $\begin{array}{l}\text { embryonic } \\
\text { human }\end{array}$ & ATCC® CRL- $1573^{\mathrm{TM}}$ & DMEM & 10 \\
\hline HeLa & $\begin{array}{l}\text { cervix carcinoma, } \\
\text { human }\end{array}$ & ATCC® CCL- $2^{\mathrm{TM}}$ & RPMI & 10 \\
\hline SH-SY5Y & neuroblastoma, human & ATCC® CRL-2266 ${ }^{\mathrm{TM}}$ & DMEM/F-12 & 10 \\
\hline SKBR3 & $\begin{array}{l}\text { breast carcinoma, } \\
\text { human }\end{array}$ & ATCC $®$ HTB- $30^{\mathrm{TM}}$ & DMEM & 10 \\
\hline AAV-293 & $\begin{array}{l}\text { HEK-293 transformed } \\
\text { with human adenovirus } \\
\text { type } 5 \text { DNA }\end{array}$ & $\begin{array}{l}\text { Agilent Technologies, } \\
\text { USA }\end{array}$ & DMEM * & 15 \\
\hline $3 \mathrm{~T} 3$ & fibroblasts, murine & $\begin{array}{l}\text { a gift from Felix B. En- } \\
\text { gel, University Medical } \\
\text { Center Erlangen }\end{array}$ & DMEM & 10 \\
\hline
\end{tabular}

* supplemented with $1 \mathrm{mM}$ sodium pyruvate and $1 \mathrm{mM}$ L-glutamine.

\subsubsection{Primary material}

Cortex tissue was gained from animal euthanisations for approved experiments, where the cortex was not used for the project. Rodent material was given from Camin Dean's group (European Neuroscience Institute, Göttingen) and NHP material from the German Primate Center (Göttingen).

Mesenchymal stem cells, isolated from the Wharton's jelly of the human umbilical cord, 
were gained from a BMBF project cooperation with the Hemocentro Distrital (Bogota, Colombia). The anonymized donors approved the use of the MSCs for research.

Table 15: MSC donors

\begin{tabular}{|c|c|c|}
\hline $\begin{array}{l}\text { Anonymised } \\
\text { number }\end{array}$ & donor & Sex \\
\hline \#83 & & female \\
\hline$\# 97$ & & female \\
\hline \#108 & & male \\
\hline
\end{tabular}

\subsubsection{Primary cortical cultures}

Primary cortical cultures were prepared according to Shimada et al. (1969) and Fischbach (1972) with minor modifications. For a co-cultivation of neurons and glia cells cortices of E18 Wistar rats and Callithrix jacchus, Macaca fascicularis and Macaca mulatta of different ages were used.

Dissociated cultures The cortices were freed from meninges and cut into small pieces in ice-cold dissection media followed by 30 min incubation in trypsin/EDTA $(0.05 / 0.02 \%)$. Afterward, the tissue was washed with dissection media and the cells were singularized by trituration in first-day media. The cell suspension was filtered through a $100 \mu \mathrm{m}$ cell strainer (Greiner Bio-One, Germany) and the cell number counted with Trypan Blue. 40,000 to 80,000 cells in $500 \mu$ l first-day media were seeded in 24-well plates with PEItreated $(0.05 \%$ for $5 \mathrm{~h})$ glass cover slips. Cells were cultured at $37^{\circ} \mathrm{C}$ with $5 \% \mathrm{CO}_{2}$ in a humidified atmosphere. After one day media was changed to neurobasal media and at day seven Ara-C was added to a final concentration of $5 \mu \mathrm{M}$ to prevent mitotic growth of glia cells.

Organotypical brain slices The cortices were freed from meninges in ice-cold dissection media and pieces of the favored area were prepared by using forceps and scalpel. Whatman paper (sterilized in $70 \% \mathrm{EtOH}$ and dried) was placed on the desk of the tissue slicer, pre-wet with dissection media. The tissue was placed on it and sliced into 300-400 $\mu \mathrm{m}$ thick slices. Up to six slices were cultivated on a membrane inlet (CatNo. PICMORG 50, Merck Millipore, USA), placed on $1.1 \mathrm{ml}$ of dissection media with glucose. Media was changed every 2-3 days. 
Table 16: Dissection media

\begin{tabular}{ll}
\hline Substance & Concentration \\
\hline Glucose & $33 \mathrm{mM}$ \\
HEPES & $10 \mathrm{mM}$ \\
HBSS & \\
\hline
\end{tabular}

Table 17: First-day media

\begin{tabular}{ll}
\hline Substance & Concentration \\
\hline FCS & $10 \%(\mathrm{v} / \mathrm{v})$ \\
GlutaMAX & $2 \mathrm{mM}$ \\
$\mathrm{P} / \mathrm{S}$ & $1 \mathrm{X}$ \\
DMEM & \\
\hline
\end{tabular}

Table 18: Neurobasel media

\begin{tabular}{ll}
\hline Substance & Concentration \\
\hline B27 supplement & $2 \%(\mathrm{v} / \mathrm{v})$ \\
GlutaMAX & $2 \mathrm{mM}$ \\
P/S & $1 \mathrm{X}$ \\
Neurobasal media & \\
\hline
\end{tabular}

\subsubsection{Lipotransfection of cells}

For lipotransfection of cells grown in a 24-well plate, the transfection conditions were as follows: per well $50 \mu$ l of pre-warmed OptiMEM were blended with $1 \mu$ l Lipofectamin 2,000 , another $50 \mu \mathrm{l}$ was blended with 0.1 to $1 \mu \mathrm{g}$ of DNA. After an incubation of $5 \mathrm{~min}$, both mixtures were combined and incubated for another $20 \mathrm{~min}$.

The transfection mixture was added dropwise to the cells, and the plate was gently shaken 
to distribute the transfection agent. Depending on the DNA construct the expression could be expected after one day of cultivation.

\subsection{5 rAAV production}

For a small scale production a $15 \mathrm{~cm}$ tissue culture dish cell+ (CatNo. 83.1803, Sarstedt, Germany) was used and for large scale production a Thermo Scientific ${ }^{\mathrm{TM}} \mathrm{Nunc}^{\mathrm{TM}}$ EasyFill $^{\mathrm{TM}}$ Cell Factory ${ }^{\mathrm{TM}}$ Systems (Thermo Fisher Scientific, USA) was used. This protocol gives the values for the production in $15 \mathrm{~cm}$ tissue culture dishes and the values for the production calculated for one layer of a Nunc $^{\mathrm{TM}}$ EasyFill $^{\mathrm{TM}}$ Cell Factory ${ }^{\mathrm{TM}}$ System in brackets.

At the first day of the production, $5 \times 10^{6}\left(1,8 \times 10^{7}\right)$ AAV-293 cells of maximal passage 10 were seeded in $30 \mathrm{ml}$ DMEM $(107 \mathrm{ml})$ and cultivated for two days.

At the third day, 73,6 $\mu \mathrm{g}(263.48 \mu \mathrm{g})$ helper\&packaging plasmid (Plasmid Factory, Germany) and $18,4 \mu \mathrm{g}(65.87 \mu \mathrm{g}) \mathrm{rAAV}$ plasmid were mixed in a $50 \mathrm{ml}$ conical tube with $1.109 \mathrm{ml}(4 \mathrm{ml}) 500 \mathrm{mM} \mathrm{CaCl} 2$.

Under constant shaking $1.109 \mathrm{ml}(4 \mathrm{ml})$ 2X HBS were added and mixed by producing air bubbles with the pipette. The transfection mixture was incubated for 5 min. Next, media from the cell culture was changed to $12 \mathrm{ml}$ (43 ml) DMEM-F12 (supplemented with $2 \%$ FCS and penicillin/streptomycin (100 Units/ml, $0.1 \mathrm{mg} / \mathrm{ml})$ ) and the transfection mixture was added dropwise. For the Cell Factory System, the transfection mixture was added to the DMEM-F12 before it was filled in.

After $8 \mathrm{~h}$ incubation, the media was changed to $30 \mathrm{ml}$ DMEM $(107 \mathrm{ml})$, and the cells were cultivated for another $40 \mathrm{~h}$.

At day five the rAAV comprehending cells were harvested. Therefore cultivation media was removed carefully, $0.5 \mathrm{ml} 1 \mathrm{X}$ gradient buffer was added and the cells removed with a cell scraper (Sarstedt, Germany) and collected in tubes.

For the cell factory cells were detached with $25 \mathrm{ml}$ trypsin, the cells collected, washed with media and resuspended in $1 \mathrm{X}$ gradient buffer.

Cells were lysed by freezing in liquid nitrogen or at $-150^{\circ} \mathrm{C}$ and thawed in a $55^{\circ} \mathrm{C}$ water bath. For mechanical disruption, the lysate was transferred through a 26 gauge needle (CatNo. L10101, NEOPOINT, Germany) followed by three cycles of freeze-thaw. The lysate was cooled to $37^{\circ} \mathrm{C}$ and $500 \mathrm{U}$ Benzonase per $1 \mathrm{ml}$ lysate was added, incubated for $1 \mathrm{~h}$ at $37^{\circ} \mathrm{C}$ and mixed every $15 \mathrm{~min}$.

Cell debris was pelleted by centrifugation at $3,000 \mathrm{X}$ g for $15 \mathrm{~min}$ at $4^{\circ} \mathrm{C}$. The virus con- 
taining crude cell lysate was stored at $4^{\circ} \mathrm{C}$ until use or further purification.

Table 19: 20X HBS

\begin{tabular}{ll}
\hline Substance & Concentration (M) \\
\hline $\mathrm{NaCl}$ & $2.8 \mathrm{M}$ \\
$\mathrm{Na}_{2} \mathrm{HPO}_{4}$ & $15 \mathrm{mM}$ \\
$\mathrm{HEPES}$ & $0.5 \mathrm{M}$ \\
\hline
\end{tabular}

Adjust to $\mathrm{pH} 7.1$ and sterile filter with $0.2 \mu \mathrm{m}$ filter.

\subsection{6 exo-AAV production}

The production and harvesting protocol of exo-AAVs was adapted from György et al. (2014). Recombinant HEK-AAV-CD9 $9_{\text {GFP }}$ cells were gained by transduction of HEK293 cells with the lentivirus LV-CD9 ${ }_{\mathrm{GFP}}-\mathrm{VSV}-\mathrm{G}$ (Kindly provided by Dr. Kai Böker, German Primate Center, Göttingen) and used for rAAV production when at least $80 \%$ of cells were determined by flow cytometry analysis to express GFP. Exo-AAVs were produced in HEK-AAV cells, seeded in four $15 \mathrm{~cm}$ dishes $48 \mathrm{~h}$ prior to transfection. Calcium phosphate transfection was performed in DMEM-F12 with penicillin/streptomycin and $2 \%$ FCS. Per plate $73.6 \mu \mathrm{g}$ helper\&packaging plasmid and 18.4 $\mu \mathrm{g}$ pAAV-CAG-GFP were applied. After $8 \mathrm{~h}$ the media was changed to DMEM, supplemented with penicillin/streptomycin, $2 \%$ FCS, $1 \mathrm{mM}$ sodium pyruvate and $1 \mathrm{mM}$ L-glutamine. The next day, media was changed to $10 \mathrm{ml}$ DMEM, supplemented with penicillin/streptomycin, $1 \mathrm{mM}$ sodium pyruvate, $1 \mathrm{mM}$ L-glutamine and $2 \%$ exosome-free FCS (16h at 100k X g depleted FCS) and cells were cultivated for two days.

Exo-AAVs were harvested from the media of four plates, which was depleted at $300 \mathrm{X} \mathrm{g}$ for $5 \mathrm{~min}$ and at 1000X g for $10 \mathrm{~min}$. The supernatant was centrifuged at $20 \mathrm{kX} \mathrm{g}$ for $1 \mathrm{~h}$ at $15^{\circ} \mathrm{C}$. The pellet was resuspended in $200 \mu \mathrm{l} \mathrm{DMEM}$ and the supernatant was centrifuged again at $100 \mathrm{kX} \mathrm{g}$ for $1.5 \mathrm{~h}$ at $15^{\circ} \mathrm{C}$ and the pellet as well resuspended (exo-AAV). The samples were kept at $4^{\circ} \mathrm{C}$ until use.

\subsubsection{Reference rAAVs from the Viral Vector Core}

In this thesis, later referred as purchased reference virus, we applied following viruses from the Viral Vector Core, University of North Carolina (UNC), Chapel Hill, North Carolina, 
USA.

Table 20: Standard rAAVs from the Viral Vector Core

\begin{tabular}{l} 
rAAV2/5-CaMKII-Jaws-KGC-GFP-ER2 \\
rAAV2/5-CaMKII-eNpHR3.0-mCherry-WPRE \\
rAAV2/5-CaMKII-hChR2(H134R)-eYFP-WPRE \\
rAAV2/5-SSpEMBOL-CBA-GFP \\
rAAV2/5-CaMKII-eArchT3.0-eYFP \\
rAAV2/5-CaMKIIa-hChR2(H134R)-eYFP-WPRE \\
\hline
\end{tabular}

\subsection{8 lodixanol density gradient}

To eliminate cell debris and empty rAAVs the crude cell lysate was loaded on an iodixanol gradient consisting of four different layers (Adapted from Zolotukhin et al. (1999)). The gradient was centrifuged at 104,000X g for $15 \mathrm{~h}$ at $4^{\circ} \mathrm{C}$ in a JS 24.38 rotor for Avanti J-30I (Beckman Coulter). The twelve bottom low fractions were collected in $1 \mathrm{ml}$ aliquots with the rAAVs accumulated in the $40 \%$ phase. Aliquots were stored at $-80^{\circ} \mathrm{C}$.

Table 21: Iodixanolgradient

\begin{tabular}{ll}
\hline Volume $(\mathrm{ml})$ & OptiPrep60 $(\%)$ \\
\hline 9.7 & 15 \\
6.4 & 25 \\
5.4 & 40 \\
5.4 & 58 \\
\hline
\end{tabular}


Table 22: Iodixanol gradient solutions in $\mathrm{ml}$

\begin{tabular}{lllll}
\hline & $\mathbf{1 5} \boldsymbol{\%}$ & $\mathbf{2 5} \boldsymbol{\%}$ & $\mathbf{4 0 \%}$ & $\mathbf{5 8} \boldsymbol{\%}$ \\
\hline Total & $20.5 \mathrm{ml}$ & $14 \mathrm{ml}$ & $12 \mathrm{ml}$ & $12 \mathrm{ml}$ \\
Iodixanol & 5.125 & 5.74 & 8 & 11.6 \\
$10 \mathrm{X} \mathrm{GB}$ & 2.05 & 1.375 & 1.2 & 0.4 \\
$5 \mathrm{M} \mathrm{NaCl}$ & 4.1 & - & - & - \\
$\mathrm{dd} \mathrm{H}_{2} \mathrm{O}$ & 9.225 & 6.885 & 2.8 & - \\
\hline
\end{tabular}

Table 23: 10X Gradient buffer

\begin{tabular}{ll}
\hline Substance & Concentration (M) \\
\hline Tris pH 7.6 & 1 \\
$\mathrm{NaCl}$ & 5 \\
$\mathrm{MgCl}_{2}$ & 1 \\
\hline
\end{tabular}

Fill up with $\mathrm{dd}_{2} \mathrm{O}$ and sterile filter with $0.2 \mu \mathrm{m}$ filter.

\subsubsection{Analytical iodixanol gradient}

To separate vesicles, standard rAAVs and exo-AAVs dependent on its density an analytical discontinuous iodixanol gradient with 14 fractions of increasing iodixanol concentrations from 6-60 \% in PBS was performed (With modifications from Dettenhofer and Yu (1999)). The virus samples were applied in DMEM and the gradient centrifuged for $16 \mathrm{~h}$ at $4^{\circ} \mathrm{C}$ and $103,000 \mathrm{x}$ g. The gradient was harvested in 18 fractions of $1 \mathrm{ml}$ from top to bottom.

\subsubsection{Dialysis and concentration of rAAVs}

rAAVs in iodixanol were dialysed over night in $5 \mathrm{~L} \mathrm{PBS-MK}$ at $4^{\circ} \mathrm{C}$. Therefore the dialyse membrane Spectra/Por ${ }^{\circledR}$ with a MWCO of 100,000 Da (Roth, Gemany) was used. All centrifugation steps were carried out at 3,200X g for $10 \mathrm{~min}$. The dialysed rAAV sample was centrifuged to test whether a precipitate was formed during dialysis. After this centrifugation, the sample was filled up to $20 \mathrm{ml}$ with PBS-MK, and centrifuged 
again. A Vivaspin 20 concentrator (Rettberg, Germany) with an MWCO of 100,000 Da was washed with $20 \mathrm{ml}$ PBS-MK and the pre-diluted rAAV sample was loaded on the concentrator and centrifuged until less than $1 \mathrm{ml}$ volume remained. The rAAV was washed three times with $20 \mathrm{ml}$ PBS-MK, and the last centrifugation step was prolonged until the volume decreased to less than $250 \mu \mathrm{l}$.

Table 24: PBS-MK

\begin{tabular}{ll}
\hline Substance & Concentration \\
\hline $\mathrm{NaCl}$ & $13.7 \mathrm{mM}$ \\
$\mathrm{Na}_{2} \mathrm{HPO}_{4}$ & $1.01 \mathrm{mM}$ \\
$\mathrm{KH}_{2} \mathrm{PO}_{4}$ & $1.76 \mathrm{mM}$ \\
$\mathrm{MgCl}_{2} * 6 \mathrm{H}_{2} \mathrm{O}$ & $0.05 \mathrm{mM}$ \\
$\mathrm{KCl}$ & $0.395 \mathrm{mM}$ \\
\hline
\end{tabular}

\subsubsection{Lentivirus production}

Lentiviral constructs were generated via enzymatic digestion and subsequent ligation as followed:

pLenti-hSyn-GFP was generated from pAAV-CAG-GFP (see table 43) and pLenti-hSyneNpHR3.0-eYFP (a gift from Karl Deisseroth, Addgene \#26775) with EcoRI and XbaI restriction sites.

pLenti-CAG-GFP was generated from pAAV-CAG-GFP (a gift from Karel Svoboda, Addgene \#28014) and pLenti-hSyn-eNpHR3.0-eYFP (a gift from Karl Deisseroth, Addgene \#26775) with EcoRI and PacI restriction sites.

pLenti-CaMKII-GFP was generated from pAAV-CaMKII-GFP (see table 43) and pLentiCaMKII-eNpHR3.0-eYFP (a gift from Karl Deisseroth, Addgene \#26971) with EcoRI and BamHI restriction sites.

All plasmids were checked for functionality via lipotransfection in HEK or HeLa cell lines and microscopic detection of reporter gene expression (data not shown).

For lentivirus production $1 \times 10^{6}$ HEK293FT cells were seeded in $3 \mathrm{ml}$ media per well in a 6-well plate. Right afterward the cells were transfected via calcium phosphate transfection with three plasmids: psPAX2 (a gift from Didier Trono, Addgene \#12260), pLP-VSV-G (a gift from Bob Weinberg, Addgene \#8454) and pLenti-hSyn-GFP, pLenti-CAG-GFP 
or pLenti-CaMKII-GFP. The next day, media was exchanged and centrifuged at 700X $\mathrm{g}$ and the virus containing supernatant stored at $4{ }^{\circ} \mathrm{C}$. The cell pellet was returned in the well, and sodium butyrate added. After $8 \mathrm{~h}$ and on the next day, the media was again exchanged and the supernatant collected. One day later the media was harvested, pooled with the previous collected media and filtered through $0.45 \mu \mathrm{m}$ pore size. The virus sample was stored at $4^{\circ} \mathrm{C}$.

\subsubsection{Flow cytometry analysis}

Cells were trypsinised, centrifuged at 300X $\mathrm{g}$ for $5 \mathrm{~min}$ and washed three times with PBS. The cells were fixed with $2 \% \mathrm{PFA}$ in PBS for 30 min at $4^{\circ} \mathrm{C}$ followed by three washing steps. For the measurement, the cells were resuspended in a few hundred $\mu \mathrm{l}$ of PBS. Cells were analysed with the BD LSR II system (BD, USA) the 505LP - BP530/30 filter set for GFP detection and the FACSdiva software. The data were analysed with the Flowing Software and plotted with either GraphPad Prism 6 or R.

Table 25: $4 \%$ Paraformaldehyde

\begin{tabular}{ll}
\hline Substance & Concentration \\
\hline PFA & $4 \%(\mathrm{w} / \mathrm{v})$ \\
$10 \mathrm{X} \mathrm{PBS}$ & $10 \%(\mathrm{v} / \mathrm{v})$ \\
$\mathrm{ddH}_{2} \mathrm{O}$ & $130 \mathrm{ml}$ \\
$1 \mathrm{M} \mathrm{NaOH}$ & - \\
\hline
\end{tabular}

Heat up to $60{ }^{\circ} \mathrm{C}$ under constant stirring. If it does not solve add more $\mathrm{NaOH}$. Cool down and adjust the $\mathrm{pH}$ to $7.2-7.4$. Fill up to $200 \mathrm{ml}$ and filter through $0.2 \mu \mathrm{m}$, store at $-20^{\circ} \mathrm{C}$.

Table 26: 10X PBS

\begin{tabular}{ll}
\hline Substance & Concentration (M) \\
\hline $\mathrm{NaCl}$ & 1.37 \\
$\mathrm{KCL}$ & 0.027 \\
$\mathrm{Na}_{2} \mathrm{HPO}_{4}$ & 0.091 \\
$\mathrm{KH}_{2} \mathrm{PO}_{4}$ & 0.02 \\
\hline
\end{tabular}




\subsubsection{Determination of transducing units by flow cytometry}

The concentration of transducing units of rAAV samples, the functional titer, was determined by flow cytometry analysis adapted from Kutner et al. (2009).

$6 \mathrm{X} 10^{4}$ HeLa cells per 12 -well plate well were seeded in RPMI media. The virus was diluted in six steps in a 3:5 serial dilution in OptiMEM with $2 \%$ XerumFree. $6 \mathrm{~h}$ after seeding, the media from the cell culture was replaced with $1 \mathrm{ml}$ of the diluted virus. Cells were harvested $48 \mathrm{~h}$ after transduction, prepared and analysed by flow cytometry analysis as described in 2.3.12. The percentage of GFP positive cells was used to calculate the total amount of transduced cells, assuming $6 \mathrm{X} 10^{4}$ HeLa cells. The amount of GFP expressing cells was plotted against the volume of applied virus per well, and a linear regression was applied. The $\mathrm{TU} / \mathrm{ml}$ are given by the $\mathrm{y}$-value for $1 \mathrm{ml}$.

\subsubsection{PEG precipitation of vesicles}

The protocol for PEG precipitation was adapted from Alvarez et al. (2012) and Antes et al. (2015). In brief, the vesicle containing media was depleted at $500 \mathrm{x} \mathrm{g}$ for $5 \mathrm{~min}$ and at 2,000 x g for $30 \mathrm{~min}$. The supernatant was filtered through $0.45 \mu \mathrm{m}$ filter device and mixed with 16.6 \% PEG (1:5 PEG8000 in PBS). The mixture was incubated under constant rotation at $4^{\circ} \mathrm{C}$ for $16 \mathrm{~h}$ and vesicles isolated by centrifugation at $1,500 \mathrm{X}$ g for $30 \mathrm{~min}$. The pellet was resuspended in PBS or DMEM.

\subsubsection{Nanoparticle tracking analysis}

Extracellular vesicles were analysed with the NanoSight LM10 instrument (Malvern Instruments Ltd, UK) with a recording time of $30 \mathrm{sec}$. The data were processed with the NTA software 2.3 (Dragovic et al. (2011)).

\subsubsection{VLP packaging and transduction}

VLPs consisting of the modified VP1 protein from the human JC polyoma virus were produced in Hi5 cells and provided as around $2 \mu \mathrm{g} / \mu \mathrm{l}$ concentrated aliquots in reassociation buffer by Dr. Stefan Schneider (German Primate Center, Göttingen).

Befor cargo loading, the VLPs were incubated in $1 \mathrm{X}$ dissociation buffer with $10 \mathrm{mM}$ DTT for $30 \mathrm{~min}$ to disassemble into pentamers. Around $500 \mathrm{ng}$ DNA was added and after another $30 \mathrm{~min}$ incubation the sample was dialysed over night in 10,000 MWCO Slide-A-Lizer (Thermo Scientific, Germany) in $5 \mathrm{l}$ reassociation buffer at $4^{\circ} \mathrm{C}$. Cells were transduced in OptiMEM with $2 \%$ XerumFree or the corresponding media without supplements, and it was changed to the supplemented media after over night incubation. 
Table 27: Reassociation buffer

\begin{tabular}{ll}
\hline Substance & Concentration \\
\hline HEPES & $10 \mathrm{mM}$ \\
$\mathrm{NaCl}$ & $150 \mathrm{mM}$ \\
$\mathrm{CaCl}_{2}$ & $1 \mathrm{mM}$ \\
\hline
\end{tabular}

Adjust to $\mathrm{pH} 7.5$ with $5 \mathrm{M} \mathrm{NaOH}$.

Table 28: 10X Dissociation buffer

\begin{tabular}{ll}
\hline Substance & Concentration \\
\hline HEPES pH 7.5 & $100 \mathrm{mM}$ \\
EGTA & $150 \mathrm{mM}$ \\
$\mathrm{NaCl}$ & $1.5 \mathrm{M}$ \\
\hline
\end{tabular}

\subsubsection{VLP retargeting}

For retargeting of the VLPs, loaded with the linear CAG-GFP expression cassette, the

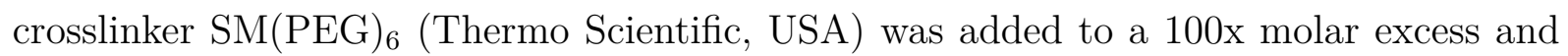
the sample incubated for $1 \mathrm{~h}$ at RT. Thereby a VLP contains 360 binding sites and $1 \mu \mathrm{g}$ equates to $7.52 \times 10^{-5} \mathrm{nmol}$. The unbound crosslinker was removed by $1 \mathrm{~h}$ dialysis against reassembly buffer in a Slide-A-Lyzer.

The peptide RVGN6 was incubated for $1 \mathrm{~h}$ at RT with $15 \mathrm{mM}$ DTT to reduce its nterminal cystein for crosslinking and subsequently the excess DTT was removed by $1 \mathrm{~h}$ dialysis in 100-500 MWCO membrane against reassembly buffer.

VLPs with crosslinker were combined with the reduced peptide in 10x molar excess and dialysed for $1 \mathrm{~h}$ in a Slide-A-Lyzer before used for transduction.

The modified retargeting protein RVGN6 based on the sequence published from Javed et al. (2016) was ordered from GeneCust (GeneCust, Luxembourg): H-Thr-Pro-Cys-AspIle-Phe-Thr-Asn-Ser-Arg-Gly-Lys-Arg-Ala-Ser-Asn-Gly-Ser-Gly-Ser-Cys-OH. 


\subsubsection{Microscopy}

Phase and fluorescence microscopy pictures were taken at the Axio Vert.A1 (Zeiss, Germany) with the filter set P 525/50 (GFP/YFP) and the filter set BP 605/70 (RFP/mCherry/tdTomat

Electron microscopy was performed using 200 mesh formvar and carbon coated copper grids (Electron Microscopy Science, USA) and observation was performed with the electron microscope EM10A/B (Zeiss, Germany). The rAAV sample, pure or diluted 1:10 in Sorensen's buffer solution with PFA, was deposited on the UV-exposed grid for 5-40 min, followed by 5 wash steps with $\mathrm{ddH}_{2} \mathrm{O}$ and $3 \mathrm{~s}$ incubation in $2 \%$ uranyl acetate. To inactivate the rAAVs the grid was exposed for 20 min to UV-light.

Table 29: Sorensen's buffer solution with PFA

\begin{tabular}{ll}
\hline Substance & Concentration \\
\hline $\mathrm{Na}_{2} \mathrm{HPO}_{4}$ & $26.75 \mathrm{mM}$ \\
$\mathrm{KH}_{2} \mathrm{PO}_{4}$ & $6.5 \mathrm{mM}$ \\
$\mathrm{PFA}$ & $4 \%$ \\
\hline
\end{tabular}

\subsubsection{Voltage clamp recording}

The voltage clamp measurement was performed together with Dr. Markus Stahlberg at the European Neuroscience Institute, Göttingen. HeLa cells were cultivated on a glass cover slip and transduced with rAAV2/5-CAG-GFP. The experiment was performed with an inverted Zeiss Axio Observer.Z1 equipped with a $100 \mathrm{~mW}$ OPSL laser module for stimulation at $488 \mathrm{~nm}$. The photocurrent response was recorded after a $500 \mu$ s pulse with $5 \%$ laser intensity at $488 \mathrm{~nm}$. For detailed information see Stahlberg (2016). 


\subsection{Protein biochemistry}

\subsubsection{Protein expression in E.coli}

Generation of pET28a $(+)$ expression vectors: pPICZ $\alpha$ A-TrkBscFv was kindly provided by Dr. Stefan Lüdtke and pPICZ $\alpha$ A-Her2neu-Streptavidin was kindly provided by Dr. Stefan Schneider. Pichia pastoris (P. pastoris) expression construct pPICZ $\alpha$ A-TrkBscFvStreptavidin was gained by overlap extension PCR with pPICZ $\alpha$ A-Her2neu-Streptavidin and primers \#503 and \#504, and pPICZ $\alpha$ A-TrkBscFv with primers \#501 and \#504. Both PCR-products were used as template in a PCR with primers \#501 and \#504. The purified PCR product was blunt-end ligated in pJet1.2/blunt (Thermo Scientific, Germany). Subsequently, pJet1.2-TrkBscFv-Streptavidin and pPICZ $\alpha$ A were digested with $\mathrm{XhoI}$ and $\mathrm{XbaI}$ and pPICZ $\alpha \mathrm{A}-\mathrm{TrkBscFv}-$ Streptavidin generated by ligation of the fragments.

pET-28a-TrkBscFv-Streptavidin was generated by enzymatic digestion of pJet1.2-TrkBscFvStreptavidin and pET28a(+) with NcoI and BamHI und subsequent ligation of the fragments.

pET-28a-TrkBscFv was generated by PCR amplification of pPICZ $\alpha$ A-TrkBscFv with primers \#605 and \#606 and subsequent blunt end ligation in pJet1.2/blunt. pJet1.2TrkBscFv and pET28a $(+)$ were digested with NcoI and BamHI and the fragments ligated. The corresponding plasmid maps are attached in the appendix.

Recombinant proteins were expressed in E.coli SoluBL2 $1^{\circledR}$ or BL21 Star with the pET$28 \mathrm{a}(+)$ vector system. The recombinant strain was used to inoculate a $3 \mathrm{ml}$ over-night culture and used at the next day to start a $50 \mathrm{ml}$ culture over night. At the day of expression, $100 \mathrm{ml}$ were inoculated with the over-night culture to an $\mathrm{OD}_{600}$ of 0.4 and grown at $37^{\circ} \mathrm{C}$ until $\mathrm{OD}_{600}$ reached around 0.6. The protein expression was induced with $0.5 \mathrm{mM}$ IPTG and the culture was harvested after several hours.

\subsubsection{Protein extraction}

Proteins expressed in E.coli were purified in small scale for an analytical expression and large scale for FPLC purification.

Whole cell protein isolation $1 \mathrm{ml}$ aliquots of the culture were centrifuged at 5,000X $\mathrm{g}$ for $10 \mathrm{~min}$. The pellet was resuspended in $50 \mu \mathrm{l} 2 \mathrm{X}$ Laemmli buffer and incubated at $95^{\circ} \mathrm{C}$

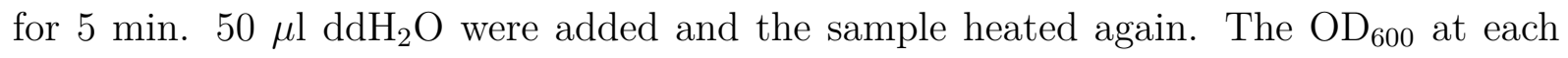
sampling time point was measured, and according to this, all samples were diluted with 1X Laemmli to an $\mathrm{OD}_{600}$ of 0.5. Before loading $5 \mu \mathrm{l}$ on a SDS-PAGE, the sample was heated up again and centrifuged for $1 \mathrm{~min}$ at 21,000X g. 
Soluble cell protein isolation $4 \mathrm{ml}$ aliquots of the culture were centrifuged at $3.000 \mathrm{X} \mathrm{g}$ for 10 minutes. The pellet was resuspended in lysis buffer (PBS with PMSF, lysozyme, $\mathrm{MgCl}_{2}$, and DNaseI) and incubated for $1 \mathrm{~h}$ on ice on a shaker. $300 \mu \mathrm{l}$ of the sample were lysed with the Bioruptor in 15 cycles of $60 \mathrm{sec}$ sonification and $60 \mathrm{sec}$ cooling break. The sample was centrifuged for 10 minutes at $21,000 \mathrm{X}$ g at $4{ }^{\circ} \mathrm{C}$ and the supernatant mixed with $6 \mathrm{X}$ Laemmli. $10 \mu \mathrm{l}$ were loaded on a SDS-PAGE.

Large scale extraction of soluble proteins was performed for FPLC purification. Therefore, $1 \mathrm{~g}$ of bacteria pellet was resuspended in $6 \mathrm{ml}$ lysis buffer and incubated for $1 \mathrm{~h}$ on ice on a shaker. The cells were disrupted by repeated passages through a microfluidizer.

The cell debris was removed by centrifuged at 54,000X g for $20 \mathrm{~min}$ at $4^{\circ} \mathrm{C}$ and the supernatant used for FPLC purification.

\subsubsection{Protein purification (FPLC)}

Recombinant proteins with a 6XHis-taq were purified with ÄKTA avant (GE Healthcare, UK) and a $1 \mathrm{ml}$ HisTrap ${ }^{\mathrm{TM}}$ excel column. The system was run with PBS and the protein was eluted with an imidazole gradient up to $300 \mathrm{mM}$ ( $\mathrm{pH} \mathrm{7.2).}$

rAAV purification was performed with the columns and buffers cited in table 40. The elution step was performed as a gradient and the fractions collected in $1 \mathrm{ml}$. The abundance of virus was tested via real-time PCR, transduction of cell lines or dot blot.

\subsubsection{TCA protein precipitation}

The protein sample was incubated with 10X RIPA buffer (150 mM sodium chloride, 1 $\%$ Triton X-100, $0.5 \%$ sodium deoxycholate, $0.1 \%$ SDS and $50 \mathrm{mM}$ Tris, $\mathrm{pH}$ 8.0) for 10 min on ice. 1 volume of $50 \%$ TCA was added to four volumes of the protein sample and incubated for another $10 \mathrm{~min}$. The tube was spun at $13000 \mathrm{rpm}$ for $5 \mathrm{~min}$ at $4^{\circ} \mathrm{C}$ and the pellet was washed three times with cold acetone. The dried pellet was dissolved in Laemmli buffer.

\subsubsection{SDS-PAGE, Coomassie and Western blot}

SDS-PAGE $1.0 \mathrm{~mm}$ cassettes (NC2010, Novex, Germany) were poured and run in XCell SureLock ${ }^{\mathrm{TM}}$ Mini-Cell Electrophorese Cell (Life Technologies, UK). $3 \mu$ l of the PageRuler Prestained Protein Ladder (Thermo Fisher, Germany) were used for determination of protein weight and the protein samples were prepared with $6 \mathrm{X}$ Laemmli buffer. The 
system was run with $1 \mathrm{X}$ electrophorese buffer for 30 min at $45 \mathrm{~V}$ and maximal A, afterward at $80 \mathrm{~V}$ until the desired protein separation.

Table 30: 5X Electrophorese buffer

\begin{tabular}{ll}
\hline Substance & Concentration \\
\hline Tris & $125 \mathrm{mM}$ \\
Glycine & $1.25 \mathrm{M}$ \\
SDS & $10 \%(\mathrm{w} / \mathrm{v})$ \\
\hline
\end{tabular}

Table 31: 10\% SDS-PAGE

\begin{tabular}{ll}
\hline Substance & Amount \\
\hline Separating gel (10\%) & \\
$\mathrm{H}_{2} \mathrm{O}$ & $3.563 \mathrm{ml}$ \\
$40 \%$ acrylamid & $1.875 \mathrm{ml}$ \\
1.5 M Tris pH 8.8 & $1.875 \mathrm{ml}$ \\
$10 \%$ SDS & $75 \mu \mathrm{l}$ \\
$10 \%$ APS & $75 \mu \mathrm{l}$ \\
TEMED & $7.5 \mu \mathrm{l}$ \\
\hline Stacking gel $(6 \%)$ & \\
$\mathrm{H}_{2} \mathrm{O}$ & $2.745 \mathrm{ml}$ \\
$40 \%$ acrylamid & $0.47 \mathrm{ml}$ \\
1 M Tris pH 6.8 & $0.47 \mathrm{ml}$ \\
$10 \%$ SDS & $37.6 \mu \mathrm{l}$ \\
$10 \%$ APS & $37.6 \mu \mathrm{l}$ \\
\hline TEMED & $3.76 \mu \mathrm{l}$ \\
\hline
\end{tabular}


Coomassie stain Proteins in the gel were stained with coomassie stain solution and excessive dye was removed in destainer solution until only the protein lanes were visible.

Table 32: Coomassie stain

\begin{tabular}{ll}
\hline Substance & Concentration \\
\hline Coomassie Blue R250 & $0.2 \%$ \\
Acetic acid & $7.5 \%$ \\
EtOH & $50 \%$ \\
\hline
\end{tabular}

Add $\mathrm{ddH}_{2} \mathrm{O}$.

Table 33: Destainer

\begin{tabular}{ll}
\hline Substance & Concentration \\
\hline EtOH & $20 \%$ \\
Acetic acid & $10 \%$ \\
\hline
\end{tabular}

Add $\mathrm{ddH}_{2} \mathrm{O}$.

Western Blot Proteins in the SDS-PAGE were transferred onto a nitrocellulose membrane (Amersham, Germany). In the module X Cell II Blot Module (Life Technologies, UK) a stack of the membrane and the nitrocellulose membrane, embedded in two layers of Whatman paper and sponges was set up and filled with Towbin buffer. The transfer was carried out in $1 \mathrm{~h}$ with $25 \mathrm{~V}$.

The membrane was blocked for $1 \mathrm{~h}$ in TBS-T with $2 \%$ milk powder, washed three times with TBS-T and incubated over night at $4^{\circ} \mathrm{C}$ with the primary antibody diluted in TBST (6x-His Tag Monoclonal Antibody 1:1000 (4E3D10H2/E3, Thermo Fisher, Germany), anti-CD9 1:500 (ab92726, Abcam, USA), anti-CD63 1:1000 (10628D, Thermo Fisher Scientific, USA), anti-Alix 1:1000 (634502, BioLegend, USA), anti-GFP 1:500 (ab38689, Abcam, USA), anti-AAV 1:1000 (60158, PROGEN Biotechnik, Germany)). The membrane was washed three times with TBS-T and incubated for $1 \mathrm{~h}$ with the secondary antibody 1:10,000 in TBS-T (HRP-coupled goat anti-Mouse secondary antibody (Life Technologies, UK), anti-rabbit (G-21234, Life Technologies, USA)). The membrane was again washed before Luminata Forte Western HRP substrate (Merck Millipore, Germany) was added 
to the membrane and HRP activity was visualized with the chemiluminescence system.

Table 34: Towbin buffer

\begin{tabular}{ll}
\hline Substance & Concentration \\
\hline Tris & $25 \mathrm{mM}$ \\
Glycin & $0.192 \mathrm{M}$ \\
$\mathrm{MeOH}$ & $20 \%$ \\
\hline
\end{tabular}

Add $\mathrm{ddH}_{2} \mathrm{O}$.

Table 35: TBS-T

\begin{tabular}{ll}
\hline Substance & Concentration \\
\hline Tris & $50 \mathrm{mM}$ \\
$\mathrm{NaCl}$ & $150 \mathrm{mM}$ \\
Tween-20 & $0.05 \%$ \\
\hline
\end{tabular}

Add $\mathrm{ddH}_{2} \mathrm{O}$. Adjust to $\mathrm{pH}$ 7.6.

\subsubsection{Silver stain of SDS-PAGEs}

Silver stain of SDS-PAGEs was performed after Heukeshoven and Dernick (1985). All solutions were prepared with fresh purified water at the day of use. The SDS-PAGE was incubated over night at $4^{\circ} \mathrm{C}$ in $150 \mathrm{ml}$ fixation solution on a platform rocker. The gel was then incubated for $2 \mathrm{~h}$ in $150 \mathrm{ml}$ reduction solution and afterward washed three times for 20 min with $\mathrm{dd}_{2} \mathrm{O} .1 \mathrm{~h}$ incubation in $125 \mathrm{ml}$ silver stain solution followed two wash steps in $\mathrm{ddH}_{2} \mathrm{O} .125 \mathrm{ml}$ developer solution was added until a brownish precipitate was formed. The gel incubated in $125 \mathrm{ml}$ fresh developer until the aimed dying intensity was reached. The dye-reaction was stopped with $125 \mathrm{ml}$ of the $0.05 \mathrm{M}$ glycine solution in $\mathrm{dd}_{2} \mathrm{O}$, which was then removed in three washing steps. 
Table 36: Fixation

\begin{tabular}{ll}
\hline Substance & Concentration \\
\hline EtOH & $30 \%(\mathrm{v} / \mathrm{v})$ \\
Acetic acid & $10 \%(\mathrm{v} / \mathrm{v})$ \\
\hline
\end{tabular}

Add $\mathrm{H}_{2} \mathrm{O}$.

Table 37: Reduction

\begin{tabular}{ll}
\hline Substance & Concentration \\
\hline $\mathrm{C}_{2} \mathrm{H}_{3} \mathrm{NaO}_{2}$ (water free) & $0.5 \mathrm{M}$ \\
Glutaraldehyde & $0.5 \%(\mathrm{v} / \mathrm{v})$ \\
$\mathrm{Na}_{2} \mathrm{~S}_{2} \mathrm{O}_{3} * 5 \mathrm{H}_{2} \mathrm{O}$ & $0.2 \%(\mathrm{v} / \mathrm{v})$ \\
\hline
\end{tabular}

Add $\mathrm{H}_{2} \mathrm{O}$.

Table 38: Silver stain

\begin{tabular}{ll}
\hline Substance & Concentration \\
\hline $\mathrm{AgNO}_{3}$ & $0.1 \%(\mathrm{w} / \mathrm{v})$ \\
Formaldehyde & $0.02 \%(\mathrm{v} / \mathrm{v})$ \\
\hline
\end{tabular}

Add $\mathrm{H}_{2} \mathrm{O}$.

Table 39: Developer

\begin{tabular}{ll}
\hline Substance & Concentration \\
\hline $\mathrm{Na}_{2} \mathrm{CO}_{3}$ & $2.5 \%(\mathrm{w} / \mathrm{v})$ \\
Formaldehyde & $0.01 \%(\mathrm{v} / \mathrm{v})$ \\
\hline
\end{tabular}

Add $\mathrm{H}_{2} \mathrm{O}$. 


\subsection{Statistics and software}

Statistics and graphical presentations were done with GraphPad Prism6 and R. Illustrations were done with ChemDraw Professional 15.1 or Microsoft PowerPoint. Plasmid maps were generated with SerialCloner2-6.

\subsection{Cortex injection and tissue staining of Macaca mulatta}

Handling of the animals and experiments were performed in compliance with the guidelines for the welfare of experimental animals issued by the European Communities Council Directive (86/609/EEC) and the laws of the Federal Government of Germany. In accordance with the German Protection of Animals Act (Tierschutzgesetz; TierSchG), all procedures were approved by State authorities (LAVES) and the Animal Welfare Office of University Medical Center Göttingen, Germany.

The craniotomy and stereotactic virus injection in the cortex of an adult Macaca mulatta was performed by Prof. Dr. Stefan Treue, Prof. Dr. Hansjörg Scherberger, Prof. Dr. Alexander Gail, Dr. Michal Fortuna and Janina Huer (German Primate Center, Göttingen). rAAV2/5-CaMKIIa-hChR2(H134R)-eYFP-WPRE (8.5x1012 vg/ml) (Viral Vector Core, University of North Carolina (UNC), Chapel Hill, USA) and rAAV2/5-CaMKIIeNpHR3.0-eYFP in $40 \%$ iodixanol $\left(7.8 \times 10^{8} \mathrm{vg} / \mathrm{ml}\right)$ were loaded into a hamilton syringe and applied with $200 \mathrm{nl} / \mathrm{min}$ for $5 \mathrm{~min}$ (total $1 \mu \mathrm{l}$ ) per injection site in the ventral premotor cortex and dorsal premotor cortex, respectively. Per injection site three deposits in Z-plane were applied. The scull was closed with the bone flap.

The animal was euthanised 10 weeks after surgery and perfused with heparanized PBS and afterwards with $4 \%$ PFA in PBS.

The immunofluorescent stainings were performed by Dr. Michal Fortuna. The frontoparietal network was processed in $50 \mu \mathrm{m}$ thick, free floating coronal slices and stained with antibodies chicken anti GFP (GFP-1020, Aves Labs, USA), Mouse Anti-NeuN Antibody (MAB377, Merck Millipore, Germany), Alexa Fluor® 488 AffiniPure F(ab') ${ }_{2}$ Fragment Donkey Anti-Chicken IgY (IgG) (H+L) (Code: 703-546-155) and Alexa Fluor® 647 AffiniPure $\mathrm{F}(\mathrm{ab})_{2}$ Fragment Donkey Anti-Mouse IgG (H+L) (Code: 715-606-150) and the images taken with an Axio Imager M2 microscope (Zeiss, Germany).

This experiment is partially described for another evaluation purpose in the submitted thesis from Huer (2018). 


\subsection{Cochlea injection and tissue staining of mice}

Handling of the animals and experiments were performed by Dr. Vladan Rankovic in compliance with the guidelines for the welfare of experimental animals issued by the European Communities Council Directive (86/609/EEC) and the laws of the Federal Government of Germany. In accordance with the German Protection of Animals Act (Tierschutzgesetz; TierSchG), all procedures were approved by State authorities (LAVES) and the Animal Welfare Office of University Medical Center Göttingen, Germany. Approximately $0.5 \mathrm{\mu l}$ virus (rAAV2/5- CAG-GFP and rAAV2/5-CaMKII-eNpHR3.0-eYFP) were injected in the right cochleas of $\mathrm{P} 6 \mathrm{C} 57 \mathrm{BL} / 6 \mathrm{~J}$ mice through the round window membrane, whereas the left cochleas were left uninjected and served as controls. The mice were euthanised 40 days after injection. Their embedded cochlea was sliced and stained against GFP/YFP (chicken anti-GFP \# ab13970, Abcam, UK; goat anti chicken 488 IgG (H+L) \# A11039, MoBiTec, Germany) for virus expression and against parvalbumin/calretinin (guinea pig anti parvalbumin polyclonal \# 195004, SYSY; rabbit anti calretinin CR 7697 \# 7697, Swant; goat-anti-guinie apig 568 \# A11075, Life Technologies, UK; alexa fluor 633 goat anti-rabbit IgG \# A21070, Thermo Fisher, Germany) for neurons and the images taken with an Axio Imager M2 microscope (Zeiss, Germany). 



\section{Results}

Gene therapy is an emerging field in research and (pre-)clinics for the cure of various diseases, which requires secure gene delivery tools and efficient gene expression.

The focus of this study lies on the delivery tools itself which can be used for any genetransfer approach. In particular, we concentrated on the delivery to neuronal cells with the exemplary application of optogenetics which can be expanded to any other application.

For the sake of simplicity, because several approaches were handled in parallel, the following chapters are arranged in the most logical order to understand and interpret the results.

\subsection{Standard rAAV production and evaluation}

rAAVs' production is simple, but its purification for in vivo application is still challenging. This drawback is even shown by the fact that new protocols are continuously published, indicating that no gold standard has yet been found. AAV5 is the most diverse serotype. In this study it was used to set up a robust pipeline which allows a high standard production and functional evaluation of rAAVs for in vivo use and which can be applied for further serotypes. rAAVs are commonly produced in human cell lines by co-transfection of the plasmids coding for the gene of interest which is located between the two ITR sequences, and the genes which code for AAV capsid and packaging proteins. For rAAV5, the vast majority of the virus is maintained within the cell and not released to the medium (Vandenberghe et al., 2010). Therefore, we chose to purify rAAVs from the cells which were harvested two days after transfection and subsequently lysed. The virus-containing crude cell lysate was low-speed centrifuged to deplete the cell debris. This virus sample can already be used for applications in vitro and ex vivo (see Figures 6, 15, 16, and 17). Each rAAV serotype has a different ability to infect a variety of cell types. For being able to reach a high transduction efficiency which also depends on the species, the target tissues or cells, it requires the use of an application specific serotype and promotor. Therefore, we produced five different serotypes (rAAV1, rAAV2, chimeric rAAV1/2, rAAV5, and rAAV6). With the rationale to allow a transduction quantitation on the single cell level 
by microscopy or by flow cytometry, we delivered the fluorescent transgene GFP. The GFP expression is driven by the constitutive CAG promotor (Hitoshi et al., 1991), the two neuron-specific promotors calcium/calmodulin-dependent protein kinase II (CaMKII, Mayford et al. (1996)) or human synapsin (hSyn, Li et al. (1993)).

As the total yield of a production might differ depending on the serotype, but might include a constant effort, we compared the transduction efficiency of equal amounts of workflow preparations. Equal numbers of five different human cell lines (SKBR3, SHSY5Y, SW480, HEK, and HeLa) were transduced with the crude lysate. The percentage of transduction events was determined via flow cytometry for GFP positive cells two days post-transduction (Figure 6).

a

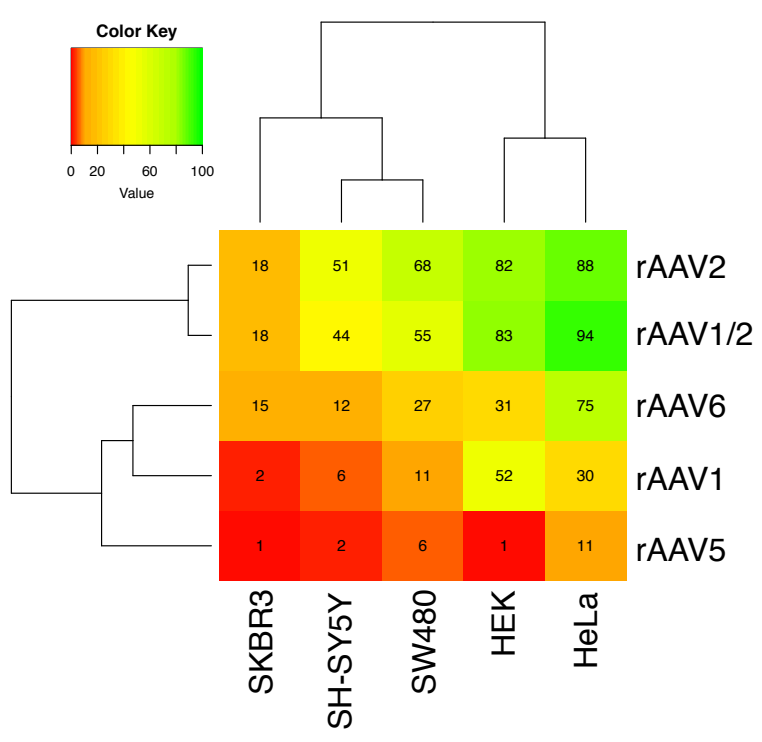

b

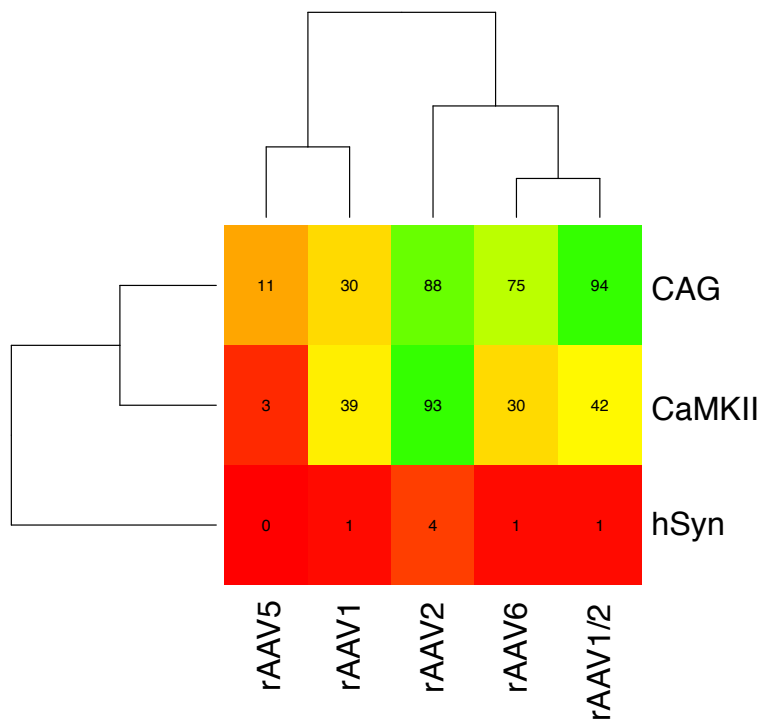

Figure 6: Transduction of five human cell lines by five rAAV serotypes with three promotors Equal amounts of workflow preparations of rAAV serotypes 1, 1/2, 2, 5 and 6 delivering the transgene for GFP were applied on human cell lines. The transduction efficency was determined via flow cytometry analysis as the percentage of GFP positive cells after $48 \mathrm{~h}$. (A) Transduction efficiency with CAG promotor driven expression. (B) Comparison of CAG, CaMKII and hSyn promotor strength of five rAAV serotypes on HeLa cells.

rAAV serotypes 2 and $1 / 2$ in combination with the CAG promotor were broadly efficient on all five cell lines, whereas serotype 5 was least efficient. All serotypes performed best on 
HeLa cells, with a maximum of $94 \%$ transduced cells for serotype $1 / 2$ and a minimum of $11 \%$ for serotype 5 (Figure 6A). SKBR3 was least efficiently transduced by all serotypes, although serotypes 1 and $1 / 2$ achieved at least $18 \%$ transduction.

Due to these findings, we chose the HeLa cell line for further analysis of rAAV performance.

Next, we wanted to elucidate which of the commonly used promotors in optogenetic constructs (CaMKII and hSyn) leads to a detectable expression in HeLa cells (Fig. 6B). The expression under the CAG promotor turned out to be the highest, with up to $94 \%$ in combination with serotype $1 / 2$. An exception was the serotype 2 , where CaMKII was slightly stronger than CAG. Comparable to the CAG promotor, CaMKII induced a suitable expression level, except for serotype 5. The hSyn promotor induced only the GFP expression in a few cells, with a maximum of $4 \%$ achieved for serotype 2 . However, the induction of the hSyn promotor was too low for subsequent analysis.

In conclusion, these data indicate that the highest transduction rates in cell culture can be achieved with the serotypes 2 and $1 / 2$ on Hela cells where constructs with the CAG and the CaMKII promotor can be used. rAAV serotype 5 performed poorly in vitro and requires an evaluation ex vivo or in vivo for applications with the CaMKII and hSyn promotor (see 3.1.3).

\subsubsection{Purification of standard rAAVs}

\section{Centrifugational purification yields a minor enrichment of rAAVs}

The crude cell lysate needs further purification, to deplete cell-derived proteins and empty viral particles and to concentrate the rAAV before it is utilised for in vivo applications. As a putative time and cost saving purification method which can be performed in any lab equipped with a tabletop centrifuge, we performed a stepwise centrifugation-based protocol. This protocol is performed in other groups to purify and to concentrate rAAVs for in vivo experiments with rodents (Protocol from Alexandra Rupp, based on personal communication with Carola Gregor, Max-Planck-Institute for Biophysical Chemistry, Göttingen).

The virus-containing crude cell lysate was sequentially centrifuged as summarized in Figure 7 and the final pellet resuspended in PBS. To visualise cell-derived and viral proteins, we loaded the samples on an SDS-PAGE and stained the proteins with coomassie. The three viral capsid proteins VP1, VP2, and, VP3 have a size of approximately $87 \mathrm{kDa}$, $73 \mathrm{kDa}$, and $61 \mathrm{kDa}$, respectively. The SDS-PAGE only showed a minor depletion effect of non-viral proteins which is why the capsid proteins could not be identified. Next, to 
check for enrichment of functional viruses, we transduced equal amounts of HeLa cells with equal volumes of the lysate, the supernatant, and the pellet and then determined the percentage of transgene expressing cells (Figure 7C and D). The final pellet was resuspended in one-tenth of the crude cell lysate volume in PBS. However, the amount of transduced cells increased only threefold and a remarkable number of the virus remained in the supernatant.

In summary, the virus enrichment did not reach our expectations of a high-quality virus that can be applied in vivo (for comparison see 3.1.2). Moreover, the coomassie stained SDS-PAGE and the controls where we loaded the crude lysate with or without containing the virus (not shown) indicated that the identification of rAAV capsid proteins in impure samples requires detection by silver stain or Western Blot (see Figure 9). 
a

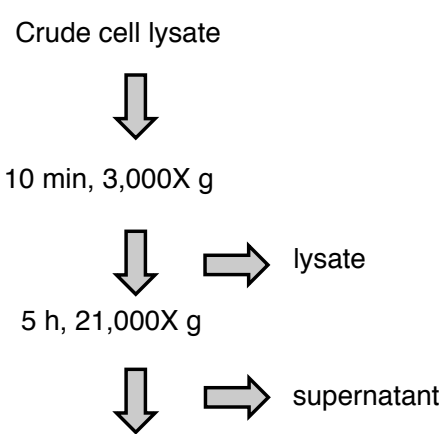

Resuspend in PBS

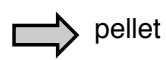

c

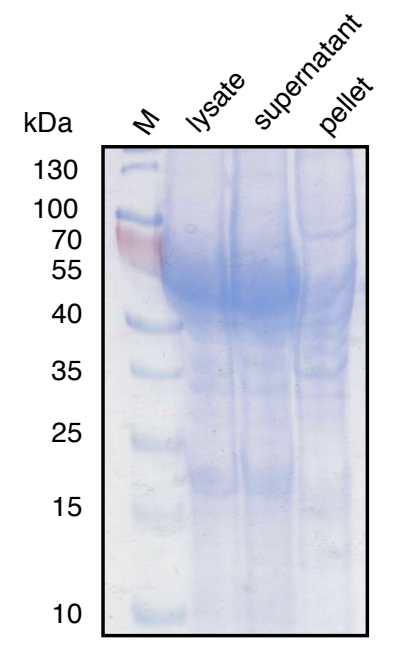

b
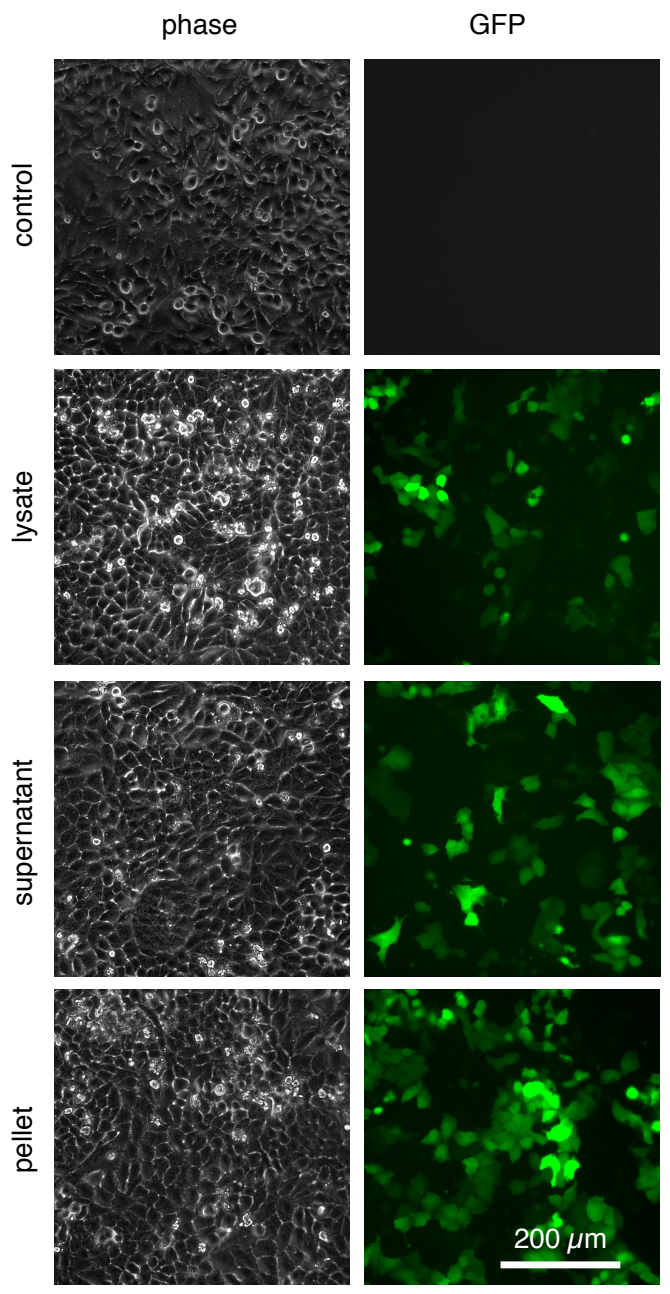

d

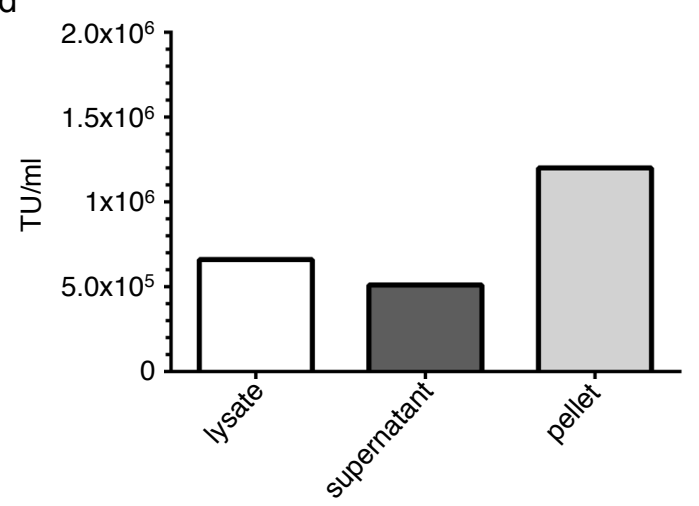

Figure 7: Sequential centrifugation protocol leads to a minor enrichment (A) Sequential centrifugation of crude lysate. (B) HeLa cells transduced with lysate, supernatant, and pellet of the purification procedure. (C) Coomassie stained SDSPAGE of crude lysate purification steps. (D) TU/ml of crude lysate purification steps determined by GFP expression. 


\section{Density gradient based purification efficiently enriches rAAVs}

Considering the different density of empty capsids and cell-derived proteins from the genome-containing viral capsids, we performed an iodixanol density gradient to reduce their amount more efficiently. The gradient consisted of four layers with iodixanol concentrations from $15 \%$ to $58 \%$. The crude cell lysate was applied on top, and the protein complexes separated during centrifugation. The gradient was harvested from top to bottom in twenty-two $1 \mathrm{ml}$ fractions (Figure 8A).

A first transduction test on Hela cells showed mentionable amounts of functional virus in fractions 13 to 18 (data not shown), of which we furthermore determined the more elaborate functional titer as well as the genomic titer. We applied serial dilutions of the virus on HeLa cells and analysed them for transgene expression. The data of technical triplicates were plotted over the applied vector genomes (vg), and a linear regression was fitted to evaluate the concentration of transducing units (for illustration see Figure 22). The genomic and functional titer correlated and had a maximum in fraction 15 with $5.46 \times 10^{8} \mathrm{vg} / \mathrm{ml}$ and $1.14 \times 10^{6} \mathrm{TU} / \mathrm{ml}$.

a

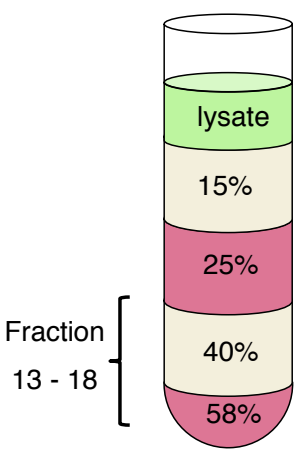

b

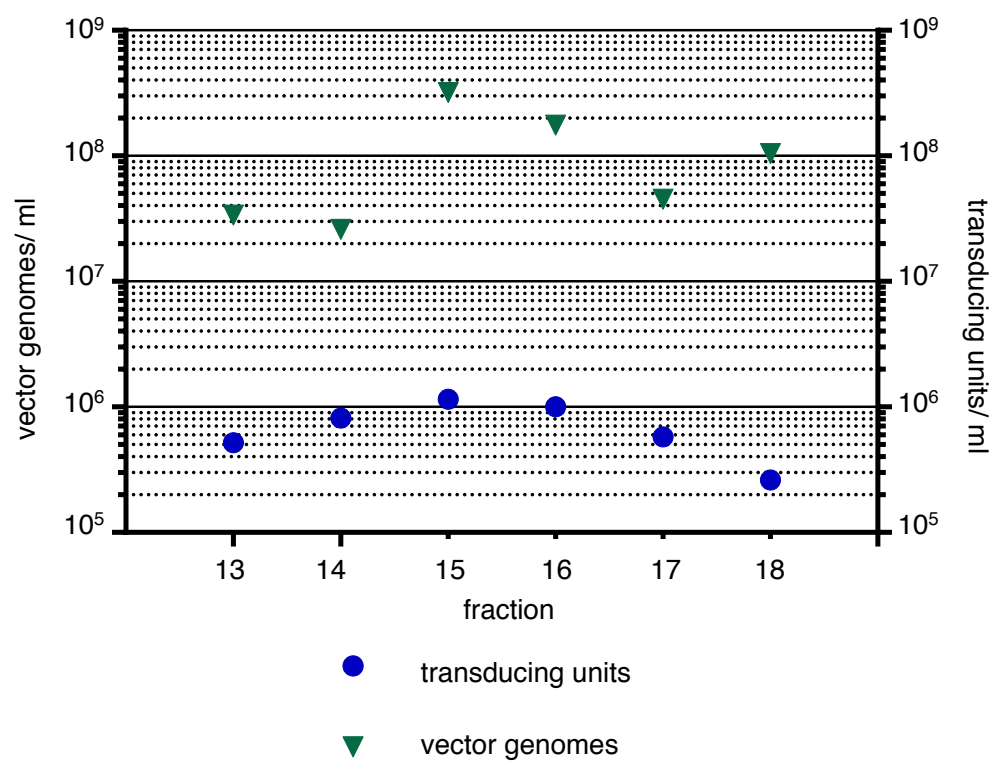

Representative values of triplicate measurement

Figure 8: rAAV2/5-CAG-GFP disribution in iodixanol density gradient: genomic and functional titer (A) Iodixanol density gradient structure with crude lysate on the top and four phases with increasing iodixanol concentrations. The gradient is harvested in $1 \mathrm{ml}$ fractions from top to bottom. (B) Genomic and functional titer of the gradient fractions 13-18. Transduction partially performed by Svenja Niehus. 
In the subsequent approach, we aimed to increase the titer of pure fractions and to exchange the iodixanol with a storage buffer for the rAAVs. Therefore, we dialysed the samples against PBS-MK and concentrated the virus with centrifugational concentrators. Equal volumes of dialysed and concentrated fractions 12-18 were then loaded on an SDSPAGE and we performed a sensitive silver stain of the proteins (Figure 9). A pure virus sample only contains the three viral capsid proteins. In our purification, fractions 13 to 18 contained the three viral protein bands only, whereas fraction 12 showed additional bands around 20 and $27 \mathrm{kDa}$. The titer of the fractions increased up to $10^{10} \mathrm{vg} / \mathrm{ml}$.

Taken together, the iodixanol gradient fractions 13 to 18 contained functional and pure viruses and were therefore also harvested in subsequent purification rounds. Aiming to increase the titer, we also pooled them before dialysis.

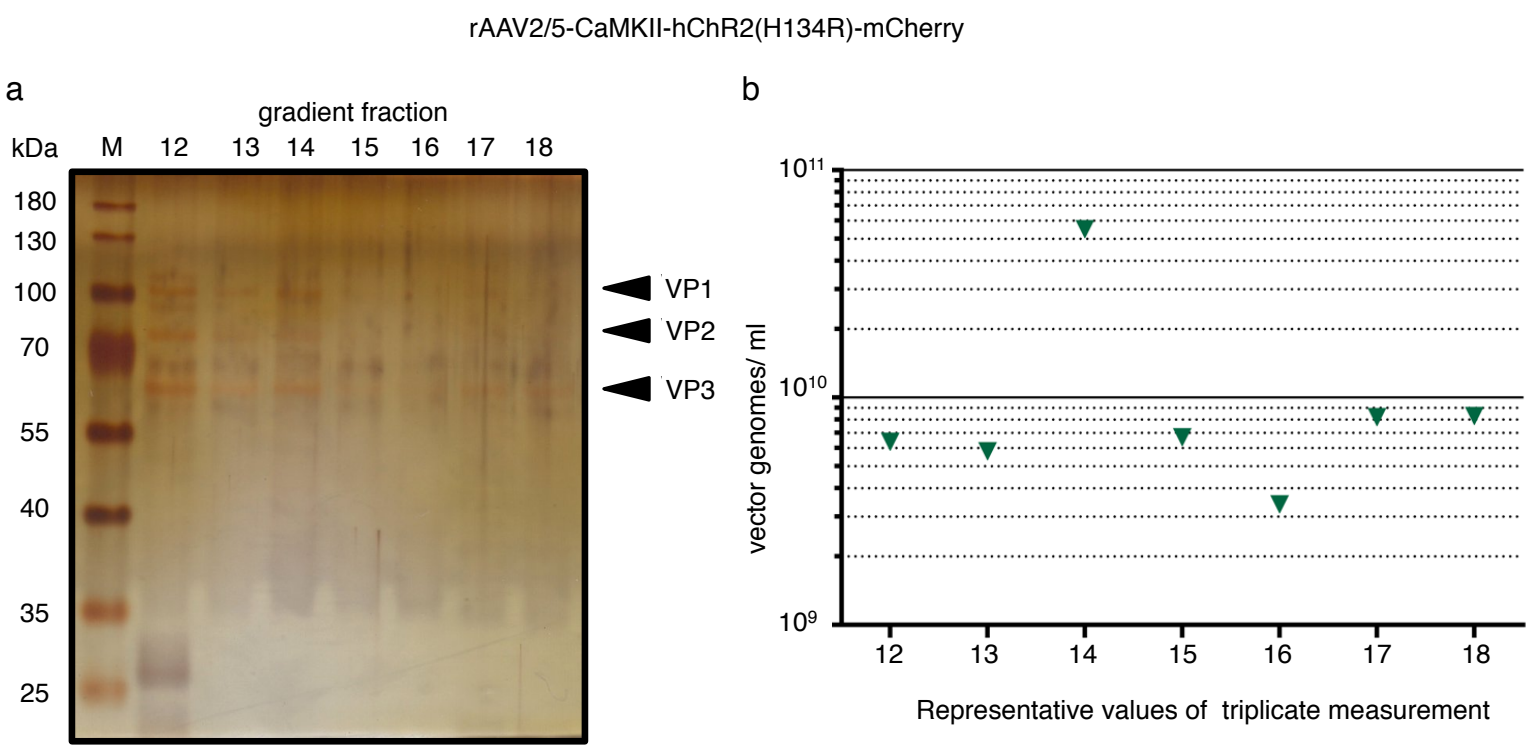

Figure 9: Purity of concentrated gradient fractions (A) Silver stained SDS-PAGE of gradient fractions 12 to 18 . (B) Genomic titer of the concentrated fractions. 


\section{rAAV column purification}

With the aim of discriminating further empty rAAV5 capsids and cell-derived proteins, we additionally tested several FPLC based protocols which were also described to be applicable for the crude cell lysate. The samples which we loaded on the columns came directly from the iodixanol gradient or were previously dialysed as summarized (Table 40).

None of the column and buffer conditions led to a detectable virus-derived peak in the chromatograms and the viral genomes were mainly found in the flow through.

Faced with the possibility that a higher number of viruses were necessary to set up the FPLC protocol and our resources were limited, we considered the previously described purification in our production range as superior.

Table 40: rAAV column purification

\begin{tabular}{lll}
\hline Column & Sample & Citation \\
\hline HiTrap $^{\text {TM }}$ Con A 4B & $\begin{array}{l}\text { dialysed iodixanol gradient } \\
\text { fractions }\end{array}$ & $\begin{array}{l}\text { Manufacturer's protocol (GE } \\
\text { Healthcare) }\end{array}$ \\
PD10 desalting column & iodixanol gradient fractions & $\begin{array}{l}\text { Manufacturer's protocol (GE } \\
\text { Healthcare) }\end{array}$ \\
MonoQ 5/50GL & $\begin{array}{l}\text { gradient fraction and dialysed } \\
\text { gradient fraction }\end{array}$ & Zolotukhin (2002) \\
HiTrap Heparin HP & $\begin{array}{l}\text { gradient fraction and dialysed } \\
\text { gradient fraction }\end{array}$ & Zolotukhin (1999) \\
\hline
\end{tabular}

\subsubsection{Quality control comparison with reference in vivo quality viruses}

\section{Virus titer and functionality}

To yield a high titer virus the iodixanol fractions 13-18 were dialysed and concentrated. Thereby the iodixanol was exchanged to PBS-MK buffer. The PBS-MK buffer allows the storage without virus precipitation, reduces the viscosity of the sample and is suitable for in vivo application. Next, we compared the virus titers and functionality with the 
reference virus.

We quantified the genomic titer by real-time PCR. However, it was demonstrated that the results of the real-time PCR depended on the location of the amplified sequence (Werling et al., 2015), and there is no standard for this method yet. Therefore, we determined the titers of three reference viruses with our real-time PCR setup and compared them with their given titers.

a

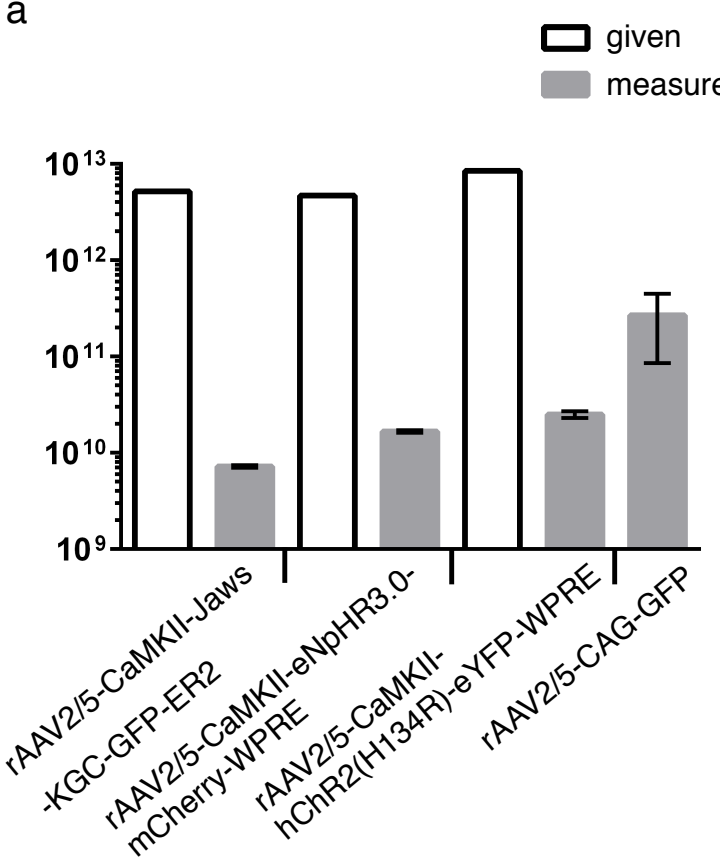

b
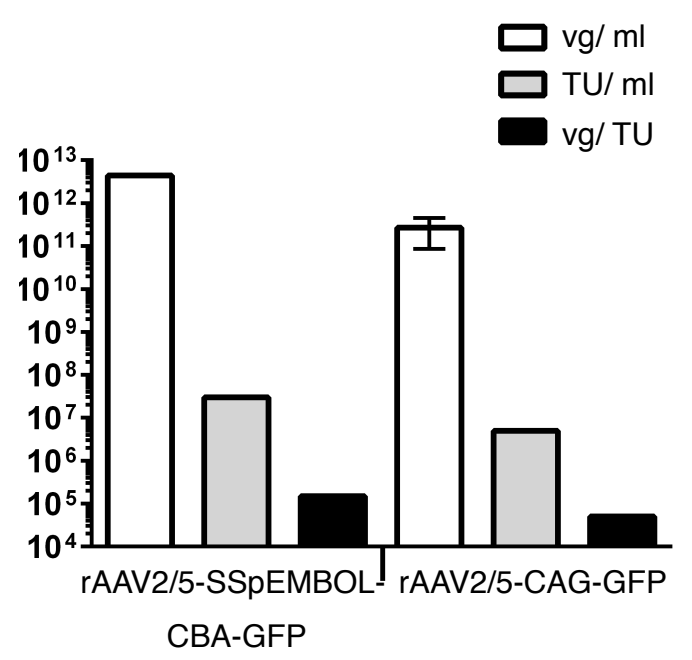

Figure 10: Genomic and functional titer of reference and self-produced viruses (A) Comparison of three reference viruses with given and measured titer with a selfproduced rAAV2/5-CAG-GFP. (B) Genomic and functional titer of comparable rAAVs from the manufacturer and self-production. Error bars in measured genomic titers indicate standard deviation of triplicate measurements.

The three reference viruses had a given genomic titer of $10^{12} \mathrm{vg} / \mathrm{ml}$. Measured with our real-time PCR setup, the titer was approximately two magnitudes lower (Figure 10). Our self-produced rAAV2/5-CAG-GFP with a titer of $10^{11} \mathrm{vg} / \mathrm{ml}$ is thereby one magnitude higher compared to the reference viruses.

Next, we compared the functional titer. For that purpose, we used the most suitable and available reference, which was rAAV2/5-SSpEMBOL-CBA-GFP. It also contains a constitutive promotor for GFP expression but lacks the WPRE sequence which prevented the quantification of the genomic titer. The self-produced virus had a titer of $5 \times 10^{6} \mathrm{TU} / \mathrm{ml}$ 
and the reference virus $3 \times 10^{7} \mathrm{TU} / \mathrm{ml}$ (Figure 10B).

The genomic and the functional titer get better the higher they are, whereas the ratio of $\mathrm{vg} / \mathrm{TU}$ describes the functionality of a viral batch and a lower ratio characterises a higher functional virus. Compared with the given titer of the reference virus, the self-produced virus is one magnitude lower, meaning higher functionality. Assuming the genomic titer of the reference to be up to two magnitudes lower, this ratio reverses. Thus there is no absolute comparison possible we can finally conclude to have a virus in the same range of functionality.

In short, we set up the quantification of the genomic and the functional titer of rAAVs and concluded that we produced a functional virus with a high genomic titer.

\section{Silver stain for the visualisation of proteins}

After checking the titer and functionality of our virus production, we compared its purity with the reference virus. For that purpose, equal sample volumes were loaded on an SDS-PAGE and proteins were visualised with a silver stain (Figure 11).

The capsid proteins could thereby be visualized with a minimum of $7.5 \times 10^{9} \mathrm{vg}$ per lane (data not shown). Only the expected three bands in the size of the viral capsid proteins were visible for the reference virus (Figure 11A). The self-produced high titer viruses rAAV2/5-CAG-GFP $\left(3 \times 10^{11} \mathrm{vg} / \mathrm{m}\right)$ and rAAV2/5-CaMKII-eNpHR3.0-eYFP $\left(4 \times 10^{11} \mathrm{vg} / \mathrm{m}\right)$ had additional bands (Figure 11B). The first had one protein band of approximately $20 \mathrm{kDa}$ (indicated by the arrow) whereas the other had several additional bands.

Despite the harvest of the previously selected fractions 13 to 18, not all co-purified proteins could be depleted. The enrichment of them might correlate with the volume of crude cell lysate that was loaded on the iodixanol gradients. In spite of everything, we showed that

our purification could greatly enrich the virus with only minor impurities which need to be identified. 
a

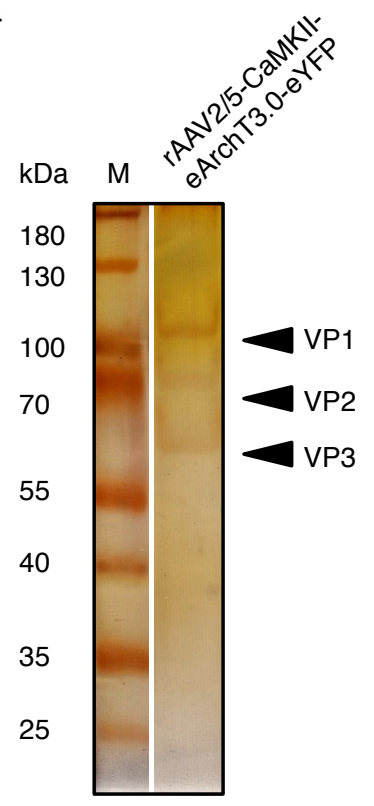

b

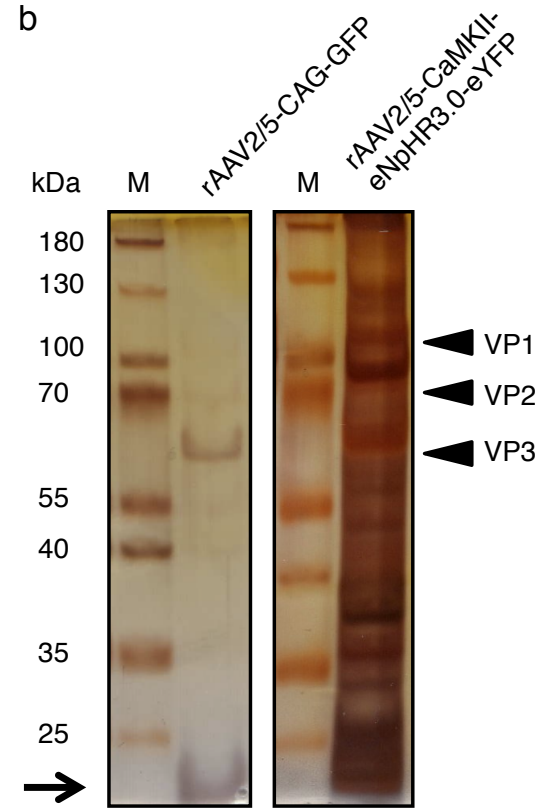

Figure 11: Silver stained SDS-PAGES for purity evaluation of rAAV samples (A) Reference virus. (B) High titer viruses from own productions. Arrow indicates the co-purified protein of approximately $20 \mathrm{kDa}$. M: Marker

\section{Electron microscopy for differentiation between full and empty capsids}

Electron microscopy of negative stained rAAV samples was utilised to quantify the distribution of filled and empty capsids (Figure 12). Each rAAV production yields capsids which are not successfully loaded with a viral genome and these empty capsids cannot be completely depleted by the purification. That is why a high percentage of filled capsids is another quality feature of a purification.

Based on the low number of viral particles on our micrographs we give a rough calculation of the distribution of empty and filled particles which is sufficient for our purpose (Figure 12). $37 \%$ of the capsids of the reference virus (rAAV2/5-SSpEMBOL-CBA-GFP) were filled and $39 \%$ of rAAV2/5-CAG-GFP and $41 \%$ of rAAV2/5-CaMKII-eNpHR3.0-eYFP from the own production (see also Figure 11). Additionally to the viral capsids with approximately $25 \mathrm{~nm}$ in diameter (Walters et al., 2004), donut shaped particles with approximately $10 \mathrm{~nm}$ in diameter were visible in both own productions (indicated by the arrow).

Taken together, the viral samples only differ a little compared to the percentage of filled 
particles, and we can conclude that they, based on this, have the same quality. However, the self-produced viruses bear a co-purified, small protein complex. 

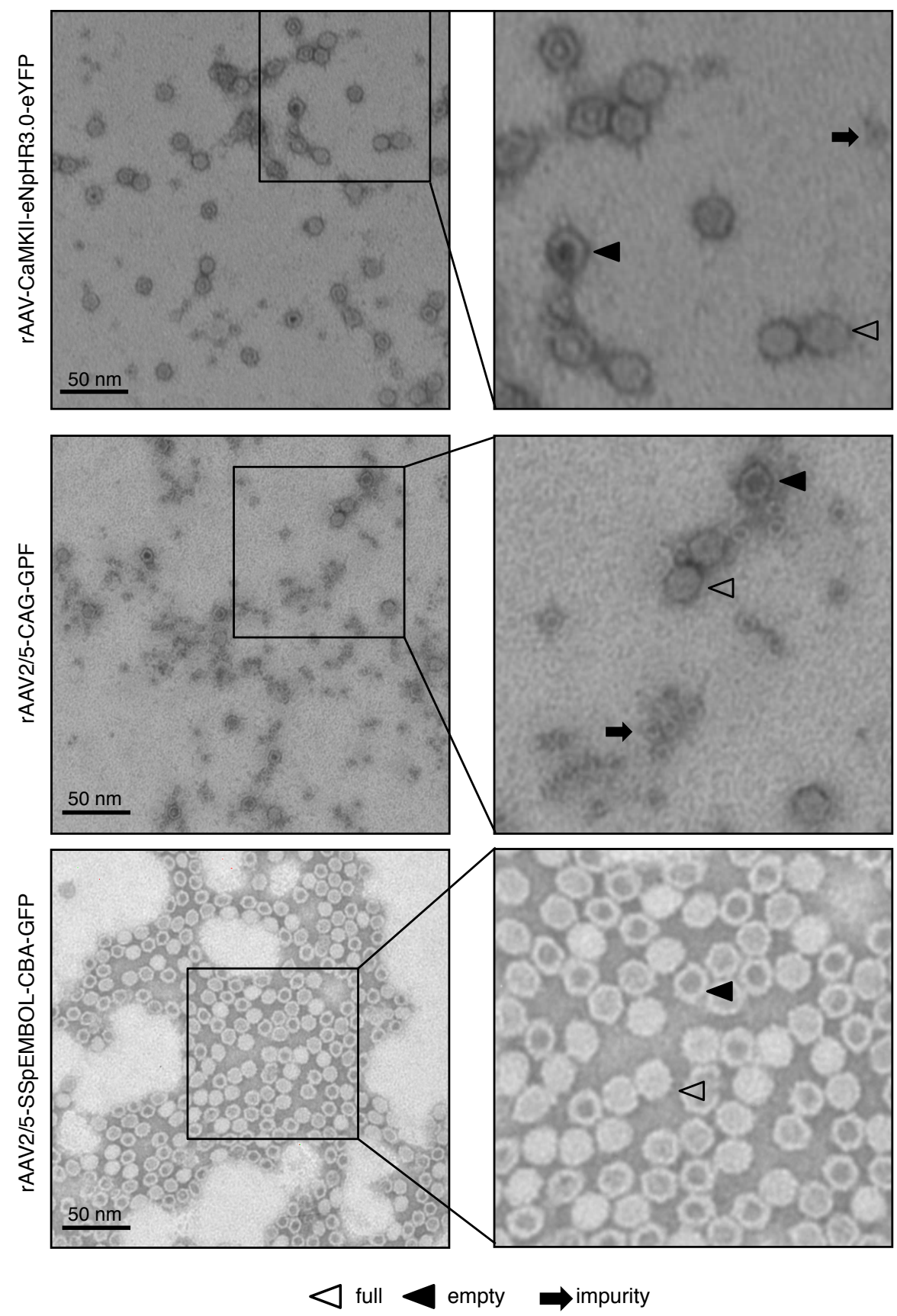

Figure 12: Visualisation of rAAV capsids by electron microscopy rAAV samples were negatively stained and fixed via UV-light exposure. All samples bear round particles of $25 \mathrm{~nm}$ in size, which correspond to genome filled (white arrow head) and empty (black arrow head) rAAV capsids. Micrographs of self-produced rAAVs show additional particles with around $10 \mathrm{~nm}$ in diameter (arrow). 


\section{Mass spectrometry identifies co-purified proteins}

Silver stain and electron microscopy indicated a co-purified impurity which we characterised as a donut-shaped protein complex consisting of around $20 \mathrm{kDa}$ proteins. Using mass spectrometry, we identified the proteins in the gel piece of rAAV2/5-CAG-GFP, located at the height of about $20 \mathrm{kDa}$.

Mass spectrometry analysis was performed in Prof. Dr. Henning Urlaub's lab for bioanalytical mass spectrometry (Max-Planck-Institute for Biophysical Chemistry, Göttingen) and the results BLASTed against all entries in UniProt (Table 45). The top ten of a total 134 hits are summarized in Table 41, with the three most abundant proteins: ferritin light chain, trypsin, and ferritin heavy chain.

Table 41: Top ten mass spectrometry hits

\begin{tabular}{llll}
\hline Number & Identified proteins & $\begin{array}{c}\text { Molecular } \\
\text { weight }\end{array}$ & $\begin{array}{c}\text { Total of } \\
\text { spectra }\end{array}$ \\
\hline 1 & Ferritin light chain (Homo sapiens) & $20 \mathrm{kDa}$ & $0.44 \%$ \\
2 & Trypsin (Sus scrofa) & $24 \mathrm{kDa}$ & $0.37 \%$ \\
3 & Ferritin heavy chain (Homo sapiens) & $21 \mathrm{kDa}$ & $0.33 \%$ \\
4 & Keratin type II cytoskeletal 1 (Homo sapiens) & $66 \mathrm{kDa}$ & $0.19 \%$ \\
5 & Keratin type II cytoskeletal 2 epidermal (Homo sapiens) & $65 \mathrm{kDa}$ & $0.13 \%$ \\
6 & Keratin type I cytoskeletal 10 (Homo sapiens) & $59 \mathrm{kDa}$ & $0.12 \%$ \\
7 & Keratin type I cytoskeletal 9 (Homo sapiens) & $62 \mathrm{kDa}$ & $0.089 \%$ \\
8 & Proteasome subunit beta type-5 (Homo sapiens) & $28 \mathrm{kDa}$ & $0.089 \%$ \\
9 & Ferritin light chain (Bos taurus) & $20 \mathrm{kDa}$ & $0.059 \%$ \\
10 & Tubulin beta-4 chain (Xenopus laevis) & $50 \mathrm{kDa}$ & $0.049 \%$ \\
\hline
\end{tabular}


Considering the molecular weight and shape of the impurity and because keratin and trypsin are known bias in mass spectrometry, we conclude that the co-purified protein in our viral samples is ferritin.

\section{Genetic toolbox for straightforward expression construct generation}

To provide a straightforward and quick clonation strategy, we generated a genetic toolbox inserting optimised restriction sites in rAAV plasmids. Thereby 80 combinations of promotors, opsins, and reporter genes in an rAAV2 plasmid backbone are possible, and further genes can easily be included (Table 42 and 43, supplements 5.1.1). It even allows 400 combination possibilities including the five used rAAV serotypes in this study.

Like most of the rAAV plasmids, the plasmid contains the woodchuck hepatitis virus post-transcriptional regulatory element (WPRE) between the reporter gene and the terminator (Wang et al., 2016).

All plasmids were checked for functionality via lipotransfection in HEK or HeLa cell lines and microscopic detection of reporter gene expression (data not shown).

Additionally to the rAAV production, the toolbox simplifies the production of lentiviruses and expression cassettes for VLPs as well and thereby enables an unbiased comparison of transduction efficiencies.

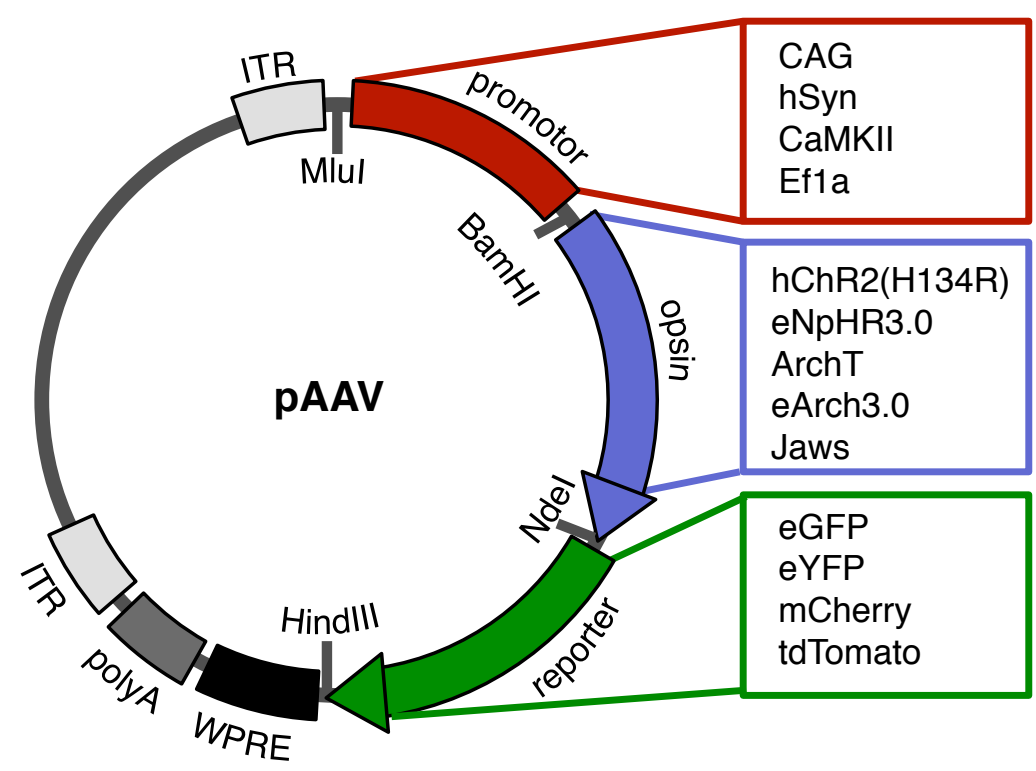

Figure 13: Schematic rAAV genetic toolbox The toolbox allows with four restriction sites (MluI, BamHI, NdeI and HindIII) 80 combination possibilities of promotors, opsins and reportergenes in an rAAV2 plasmid backbone. 
Table 42: rAAV plasmids from Addgene

\begin{tabular}{lll}
\hline Plasmid \# & Name & Source, Addgene \# \\
\hline 1 & $\begin{array}{l}\text { pAAV-CAG-hChR2(H134R)- } \\
\text { tdTomato }\end{array}$ & a gift from Karel Svoboda, 28017 \\
2 & pAAV-CAG-GFP & a gift from Karel Svoboda, 28014 \\
3 & pAAV-Ef1a-DIO eNpHR-eYFP & a gift from Karl Deisseroth, 26966 \\
4 & pAAV-CaMKII-ArchT-GFP & a gift from Edward Boyden, 37807 \\
5 & pAAV-CaMKIIa-eArch3.0-eYFP & a gift from Karl Deisseroth, 35516 \\
6 & pAAV-hSyn-hChR2(H134R)- & a gift from Karl Deisseroth, 26976 \\
7 & mCherry & \\
& pAAV-CaMKIIa-eNpHR3.0-eYFP & a gift from Karl Deisseroth, 26971 \\
\hline
\end{tabular}


Table 43: Modified rAAV plasmids

\begin{tabular}{|c|c|c|}
\hline Plasmid \# & Name & Modification \\
\hline 8 & pAAV-CaMKII-GFP & ligation of $\# 2 \& \# 5$ \\
\hline 9 & pAAV-hSyn-GFP & ligation of \#2 \& \#6 \\
\hline 10 & pAAV-Ef1a-GFP & ligation of $\# \mathbf{3} \& \# 9$ \\
\hline 11 & $\begin{array}{l}\text { pAAV-CaMKIIa-eArch3.0-NdeI- } \\
\text { eYFP }\end{array}$ & \#5 with NdeI insertion \\
\hline 12 & pAAV-CaMKII-ArchT-NdeI-GFP & \#4 with NdeI insertion \\
\hline 13 & $\begin{array}{l}\text { pAAV-hSyn-hChR2(H134R)-NdeI- } \\
\text { mCherry }\end{array}$ & \#6 with NdeI insertion \\
\hline 14 & $\begin{array}{l}\text { pAAV-CaMKIIa-eNpHR3.0-NdeI- } \\
\text { eYFP }\end{array}$ & \#7 with NdeI insertion \\
\hline 15 & pAAV-CaMKII-eNpHR3.0-GFP & ligation of \#12 \& \#14 \\
\hline 16 & $\begin{array}{l}\text { pAAV-CaMKIIa-hChR2(H134R)- } \\
\text { mCherry }\end{array}$ & ligation of $\# \mathbf{6} \& \# 7$ \\
\hline 17 & pAAV-CaMKII-Jaws-GFP & $\begin{array}{l}\text { ligation of } \# \mathbf{5} \& \text { PCR of UNC } \\
\text { rAAV2/5-CaMKII-JawsKGC-GFP- } \\
\text { ER2 }\end{array}$ \\
\hline
\end{tabular}

\section{Quick readout of opsin functionality in HeLa cells}

As a proof of concept we wanted to demonstrate that the produced virus drives a functional opsin expression and enables the generation of illumination evoked photocurrents.

We transduced HeLa cells with rAAV2/5-hChR2(H134R)-tdTomato and four days after transduction voltage clamp in combination with a $500 \mu$ s light pulse at $488 \mathrm{~nm}$ was performed to record photo currents. The light stimulation did cause no photo currents in non-transduced cells, whereas photo currents were measured in a transduced cell, identified by reporter gene expression (Figure 14).

This result confirms that we produced a functional virus which can be utilized for opto- 
gentic experiments. We furthermore point out that viruses with constructs for optogentics expressing in HeLa cells can be tested for their opsin functionality in an easy set up.

a

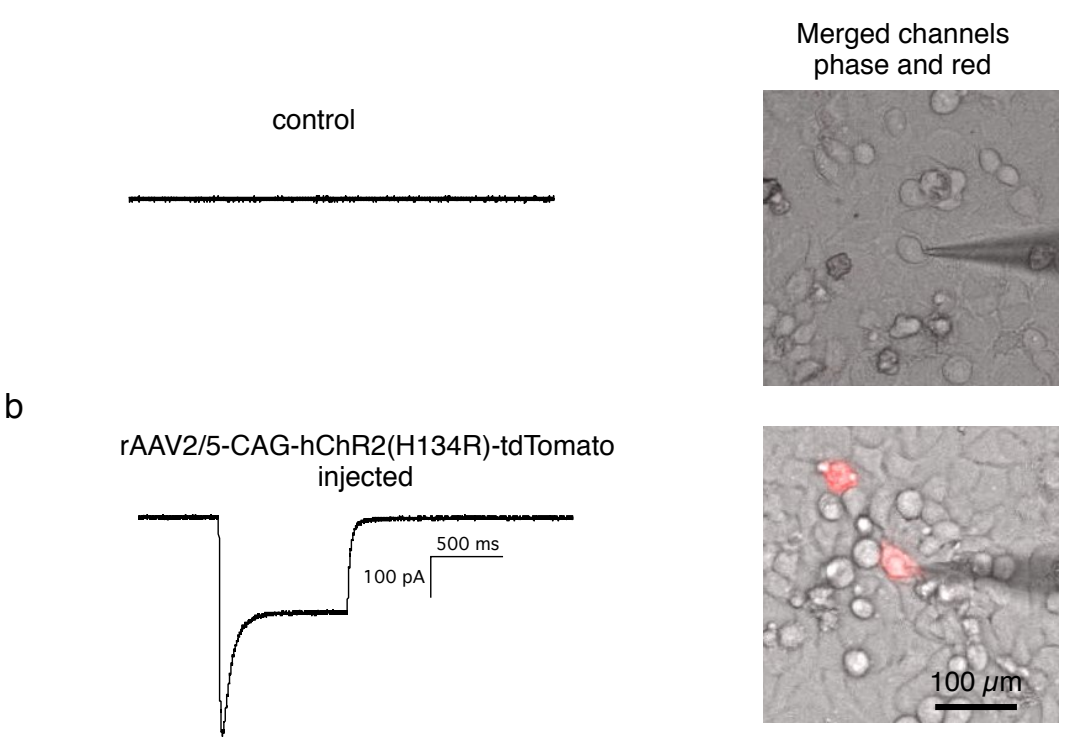

Figure 14: Illumination evoked response of light gated channelrhodopsin (A) Voltage clamp of a non-transduced HeLa cell with light stimulation. (B) HeLa cell transduced with crude lysate of rAAV2/5-CAG-hChR2(H134R)-tdTomato shows reporter gene expression. Photocurrent was recorded after a $500 \mu$ s pulse with $5 \%$ laser intensity at $488 \mathrm{~nm}$. Scale bar indicates the recorded current (vertical) and the time (horizontal). Experiment performed together with Markus Stahlberg.

\section{Summary of rAAV production, purification and quality control}

We provide a workflow for rAAV production that is independent of the serotype. We yielded a high-titer, functional virus in a good purity grade via depletion of cell-derived proteins by density gradient purification, re-buffering and concentration. The only copurified protein in our best purification can be based on its molecular weight, appearance in electron microscopy, and mass spectrometry analysis identified as ferritin.

Additionally, we characterised five rAAV serotypes for their transduction profile on a variety of human cell lines. With our genetic toolbox, we provide a straightforward way for the clonation of expression constructs for rAAVs, lentiviruses and VLPs. These can be tested in a patch clamp setup, depending on their expression in HeLa cells. 


\subsubsection{Ex vivo and in vivo applications of rAAVs}

rAAVs transduce human MSCs and cortex tissue of murine and non-human primate origin

Additionally to the production of rAAVs we wanted to elucidate to which extent the virus can be tested ex vivo before applying it in vivo.

Mesenchymal stem cells (MSCs) - In order to characterise the rAAV serotypes for their ability to transduce primary human cells, we applied them on MSCs which had been derived from the Warton's jelly of the umbilical cord (Figure 15A). Equal numbers of cells derived from three donors (\#83 female, \#97 female, and \#108 male) were transduced with equal amounts of workflow preparations and analysed for GFP-transgene expression after seven days (similar to 3.1).

a

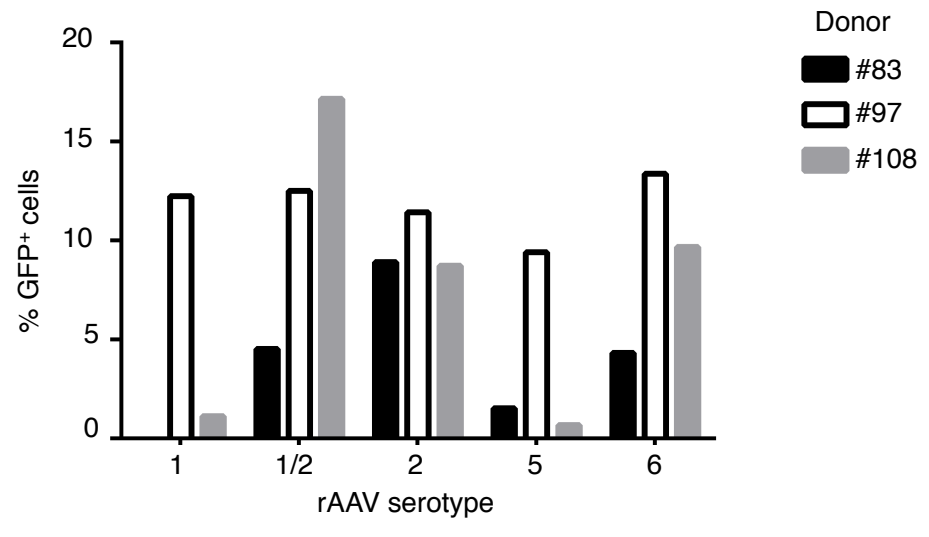

b

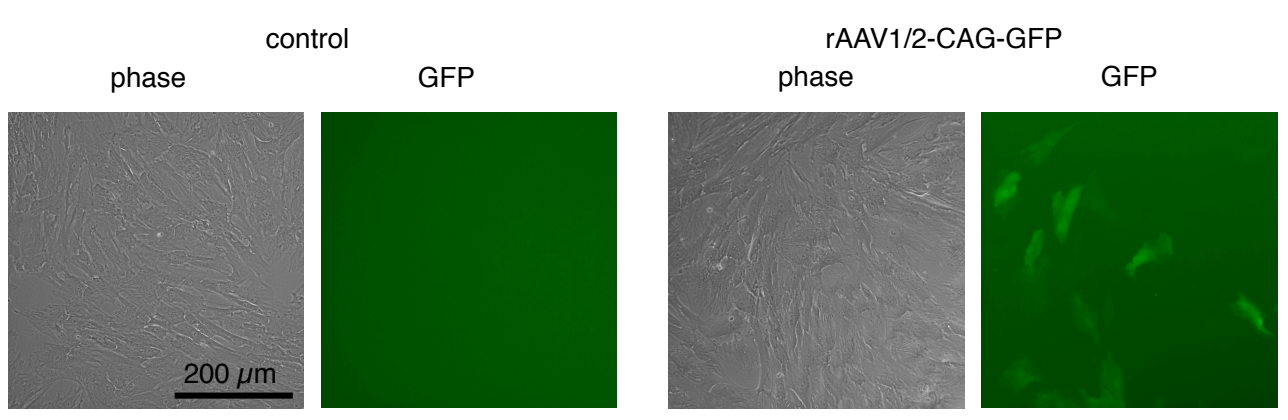

Figure 15: Human MSCs are susceptible to five rAAV serotypes with different preference (A) Equal amounts of workflow preparations of rAAV serotypes 1, 1/2, 2, 5 , and 6 delivering the transgene for GFP and the CAG promotor were applied on human MSCs. The percentage of transduced cells was determined via flow cytometry 7 days after transduction. (B) Mock transduced and rAAV2-1/2-CAG-GFP transduced MSCs of donor \#108 which showed the highest transduction. 
Based on the percentage of the GFP-expressing cells, the susceptibility of the MSCs briefly varied for the different serotypes, as well as it varied for rAAVs in general between the donors. The MSCs from donor \#97 were well susceptible to all five serotypes within a range of 10 - 15\% GFP positive cells and with rAAV 6 being superior. More interestingly, \#83's MSCs were not susceptible to serotype 1 but well transduced with serotype 2. The susceptibility of MSCs from donor \#108 highly varied in particular, being most susceptible to serotype 1/2 (Fig. 15B).

In conclusion, cultivated MSCs derived from the Warton's jelly of the umbilical cord are well susceptible for rAAVs but have a serotype preference depending on the donor.

Dissociated cortex cultures - In the next step we elucidated the serotypes' performance on dissociated cortex cultures from the Wistar rat. These cultures contained a mixed population of neurons and glia cells. Similar to the test of serotypes and promotors on human cell lines and MSCs, the cultures were transduced with equal amounts of virus-containing crude lysates. The transduced cultures were observed by fluorescence microscopy for the expression onset of GFP and micrographs were taken on day 6 in vitro (Figure 16).

rAAV serotype $1 / 2$ in combination with the CAG and the CaMKII promotor showed an expression onset just one day after transduction. But during the observation period there was no expression visible for serotype 2, and serotype 5 combined with the hSyn promotor. More interestingly, most viruses led to an expression onset on day 5 in vitro. In general, rAAV5 only transduced a few cells compared to rAAV1/2 and rAAV1 which were the most efficient viruses. Moreover, we exclusively observed expression in glia cells for serotype 5 independent of the promotor. Expression under the hSyn promotor was exclusively detected in neurons in general, whereas it was also found in glial cells for the CAG and the CaMKII promotor (morphological cell type identification).

In summary, all rAAV serotypes except rAAV2 transduced dissociated cortex cultures from the Wistar rat. More importantly, no expression in neurons was visible after transduction of rAAV5 which leads us to the conclusion that it might be due to its overall shown low transduction efficiency. 


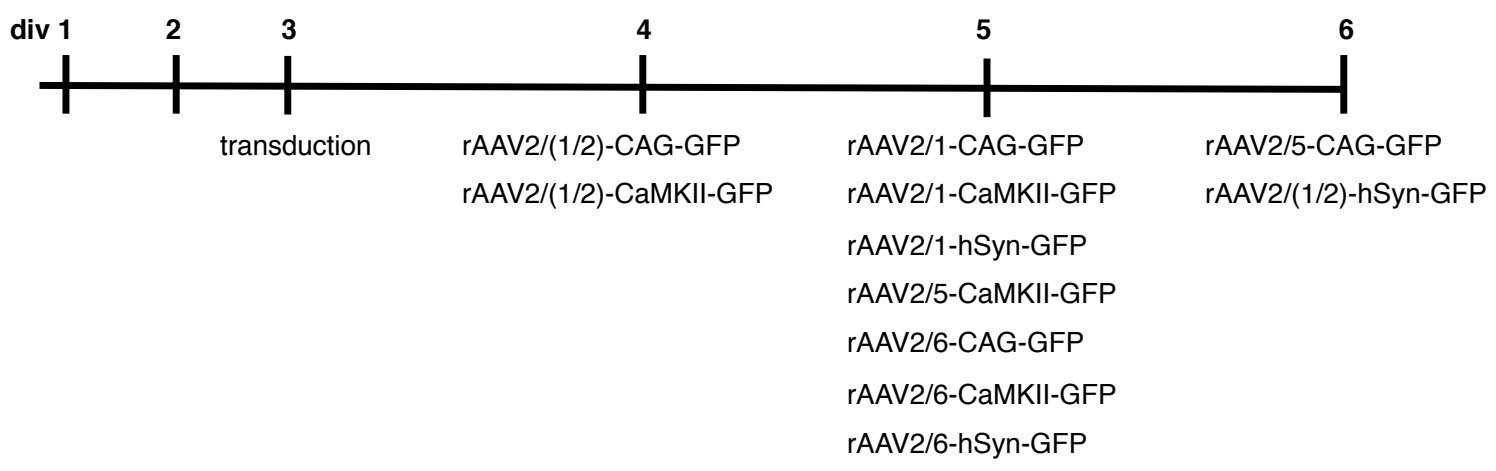

Promotor
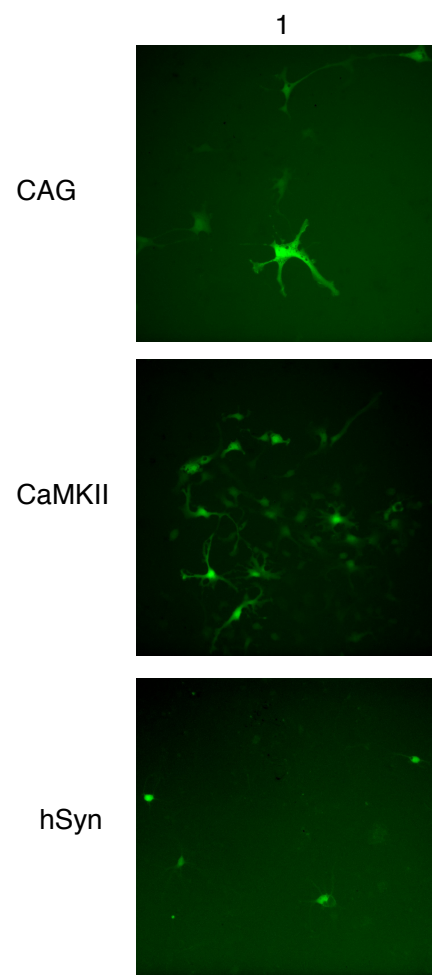
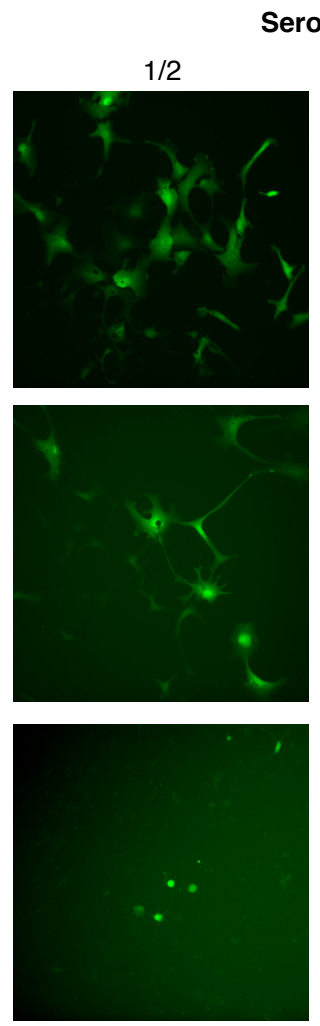

5
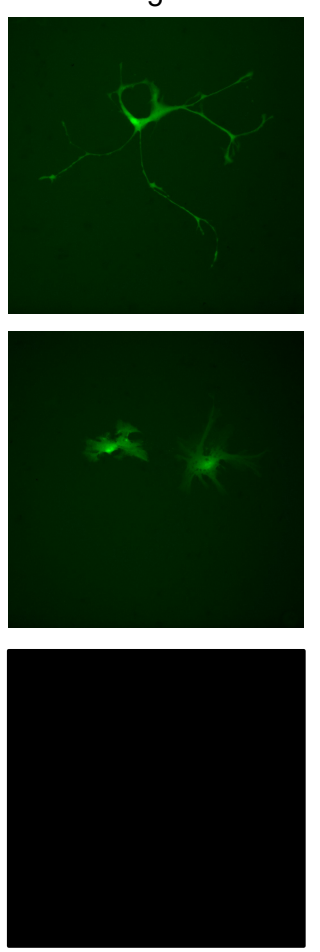

6
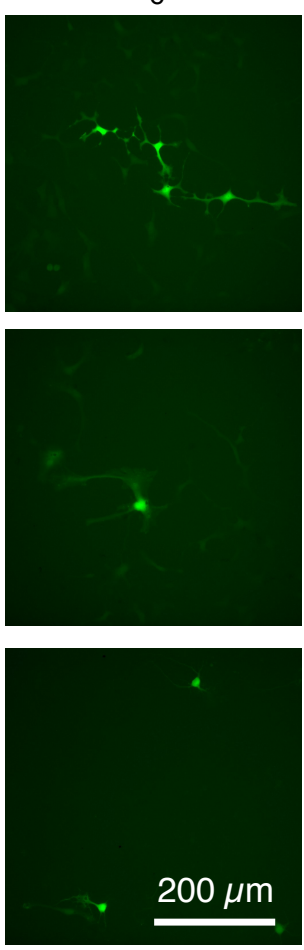

Figure 16: Expression of rAAVs in dissociated Wistar rat cortex cultures Dissociated rat cortex cultures were transduced with equal amounts of crude cell lysate of rAAV serotypes 1,2,1/2, 5, and 6 harboring an expression construct with CAG, CaMKII or hSyn promotor and GFP-transgene on day 3 in vitro (div). The vectors are itemized at the day of their first visible GFP expression. Micrographs were taken at day 6 in vitro.

Acute cortex slices - In the next step to further approach the in vivo model, we tested the transduction ability of rAAV serotype 5 on acute cortex slices of several species. We chose CNS derived from the E19 Wistar rat, the adult Macaca fascisularis, the adult Macaca mulatta, and from newborn Callithrix jacchus. 
We applied several expression constructs on the cortex slices and obtained in our study differing expression onsets in repeated experiments. In general, constructs with an opsin additionally to the reporter gene had a slower expression onset or did not lead to an expression until the cortex slice cells died. In the tissue of the non-human primates no other constructs than rAAV2/5-CAG-GFP led to an expression. Furthermore, no expression was visible on the material from Macaca mulatta before the cortex cells died.

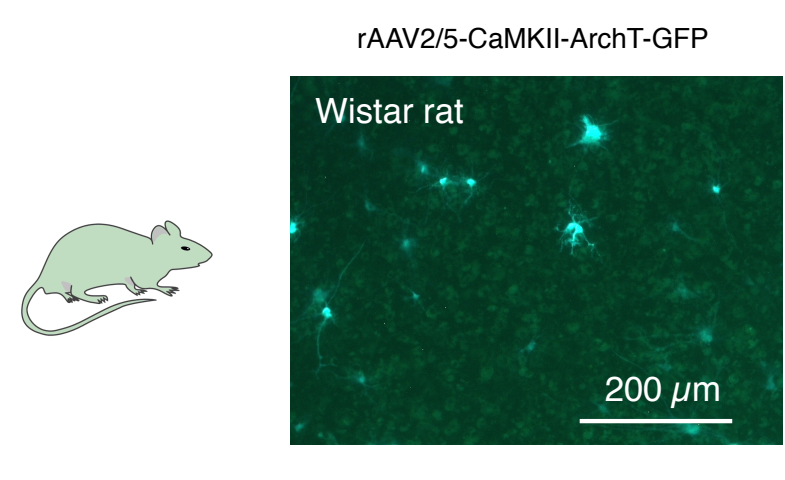

rAAV2/5-CAG-GFP

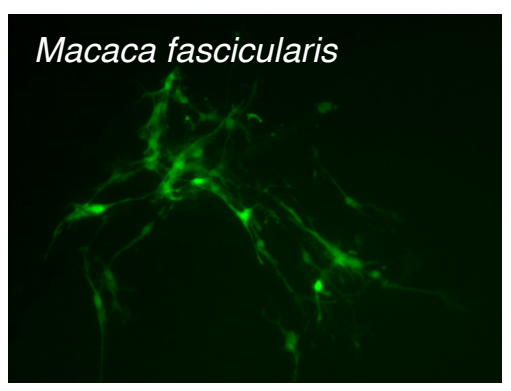

rAAV2/5-CAG-hChR2(H134R)-tdTomato

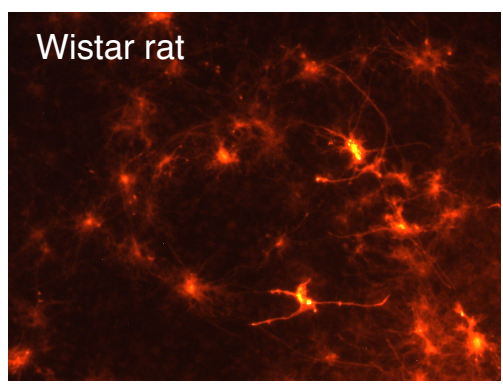

rAAV2/5-CAG-GFP

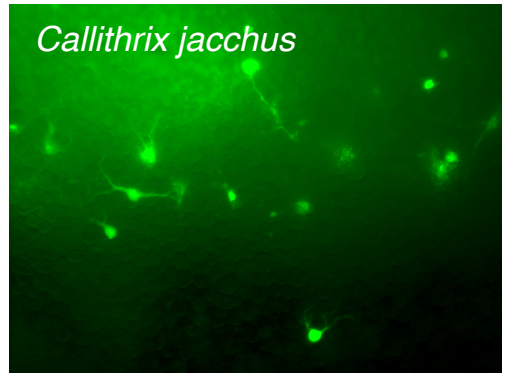

Figure 17: Expression of rAAV5 constructs in acute cortex slices of Wistar rat and non-human primate origin rAAV5 vectors were applied on acute cortex slices. The expression of the reporter gene could be detected on cultures of the Wistar rat, Macaca fascicularis and Callithrix jacchus.

From this data we conclude, that our self-produced rAAVs of the serotype 5 transduce cortex slices of rodent and non-human primate origin. But because the outcome of the experiments highly varied and the expression of opsins was uncertain, we conclude that rAAVs of the serotype 5 for opsin delivery have to be functionally tested in brain models or in vivo. Additionally, these data indicate a correlation of the culture viability and the age of the animal. We also conclude that CNS from adult non-human primates can be utilised for the culture of acute cortex slices, but have a limited usability for experiments 
which require a longer time range of several days.

\section{In vivo rAAV5 functionality}

\section{rAAV5 transduces supporting cells in the cochlea of mice}

Having shown that the rAAV serotype 5 can only be very limitedly tested ex vivo (see 3.1.3), we then evaluated its functionality in vivo. Approximately $0.5 \mu \mathrm{l}$ virus (rAAV2/5CAG-GFP and rAAV2/5-CaMKII-eNpHR3.0-eYFP, for further information see 3.1.2) were injected in the right cochleas of $\mathrm{P} 6 \mathrm{C} 57 \mathrm{BL} / 6 \mathrm{~J}$ mice through the round window membrane, whereas the left cochleas were left uninjected and served as controls. The mice were euthanised 40 days after injection. Their cochlea was sliced and stained against GFP/YFP for virus expression and against parvalbumin/calretinin for neurons (Figure 18).

The stainings visualised supporting cells (arrow), inner hair cells (asterisk) and spiral ganglion neurons (arrowhead). The whole tissue had a low fluorescence in the green channel. However, several supporting cells in both injected cochleas had an increased fluorescence. To subtract the unspecific fluorescence of the tissue and evaluate the difference of the GFP intensity, we measured the GFP intensity of spiral ganglion neuron areas and supporting cell areas of the control and the injected cochleas (Figure 18C and supplemental Figure 34). We compared the differences of the injected and non-injected animals in the fluorescence intensity of the spiral ganglion neurons with the differences in fluorescence intensity of the supporting cells. The data indicated a fluorescence above the background fluorescence for the suppoting cells for both viruses, with rAAV2/5-CAG-GFP driving a higher GFP expression than rAAV2/5-CaMKII-eNpHR3.0-eYFP.

Taken together, both viruses lead to transgene expression in several supporting cells after injection in the mice's cochleas. 
a

rAAV2/5-CAG-GFP

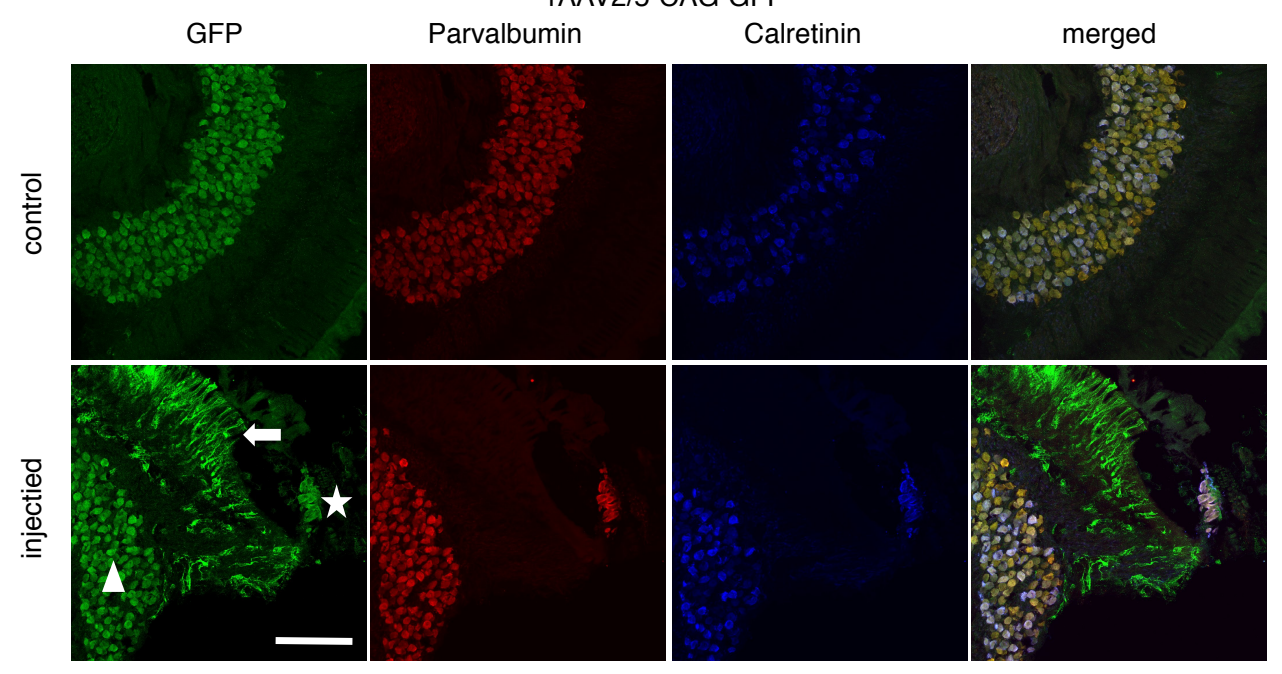

b

rAAV2/5-CaMKII-eNpHR3.0-eYFP

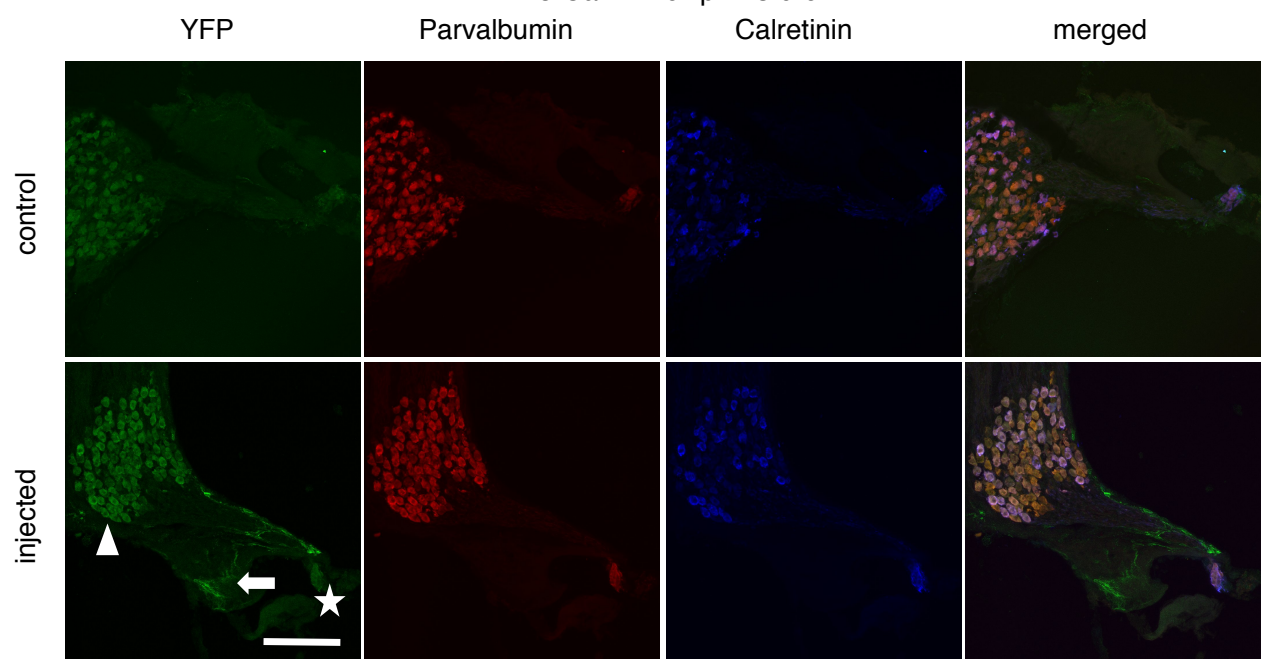

C

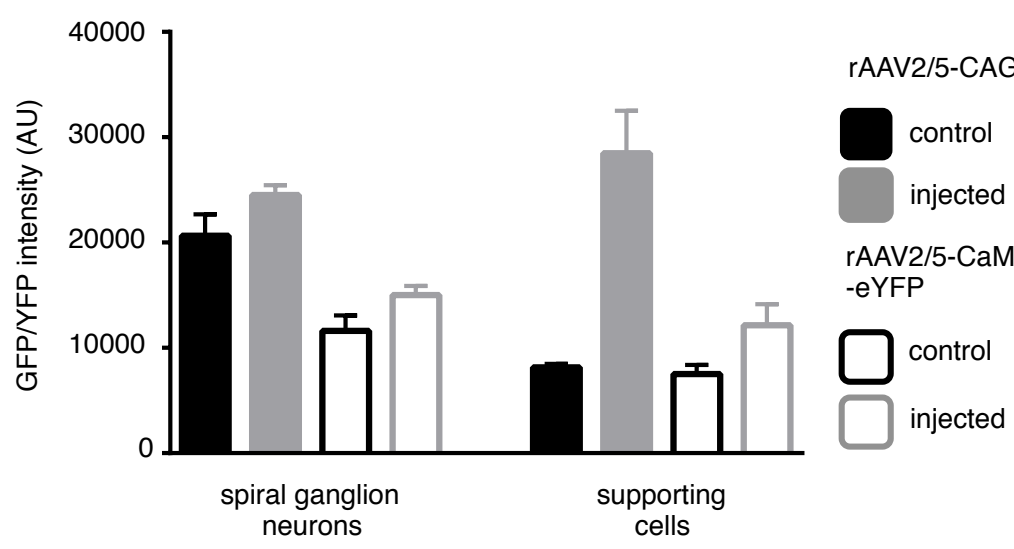


Figure 18: Expression of rAAV5 in the mouse cochlea rAAV2/5-CAG-GFP (A) and rAAV2/5-CaMKII-eNpHR3.0-eYFP (B) were injected in the right cochleas of mice, and the left uninjected cochleas served as controls. The cochleas were subsequently stained against GFP (green), parvalbumin (red) and calretinin (blue). Supporting cells (arrow), inner hair cells (asterisk) and spiral ganglion neurons (arrowhead). Scale bar $100 \mu \mathrm{m}$. (C) GFP/ YFP intensity measurement. The injection of the mice and immunofluorescence staining was performed by Vladan Rankovic and Daniela Gerke (University Medical Center, Göttingen), and micrographs were taken together.

\section{rAAV5 expression in the cortex of Macaca mulatta}

The reference virus rAAV2/5-CaMKIIa-hChR2(H134R)-eYFP-WPRE $\left(8.5 \times 10^{12} \mathrm{vg} / \mathrm{ml}\right)$ was injected in the ventral premotor cortex (PMV) and the self-produced rAAV2/5CaMKII-eNpHR3.0-eYFP (in 40\% iodixanol, $7.8 \times 10^{8} \mathrm{vg} / \mathrm{ml}$ ) in the dorsal premotor cortex (PMD), respectively, of a Macaca mulatta. The animal was euthanised ten weeks after injection and the brain regions were processed for immunofluorescence staining with antibodies against NeuN for neurons and against eYFP to identify fusionprotein-expressing cells (Figure 19B and C) (see 2.6 for experimenters).

The two, approximately $5 \mathrm{~mm}$ deep injection tracks of the reference virus were surrounded by cell bodies of eYFP expressing cells in a radius of approximately $1.5 \mathrm{~mm}$. In contrast, no injection tracks were detected within the rAAV2/5-CaMKII-eNpHR3.0-eYFP injection site. But it appeared as a wedge-shaped and sharply demarcated area of lower background fluorescence and a reduced number of NeuN positive cells in an area of approximately $2 \mathrm{~mm}$ in diameter. 
a

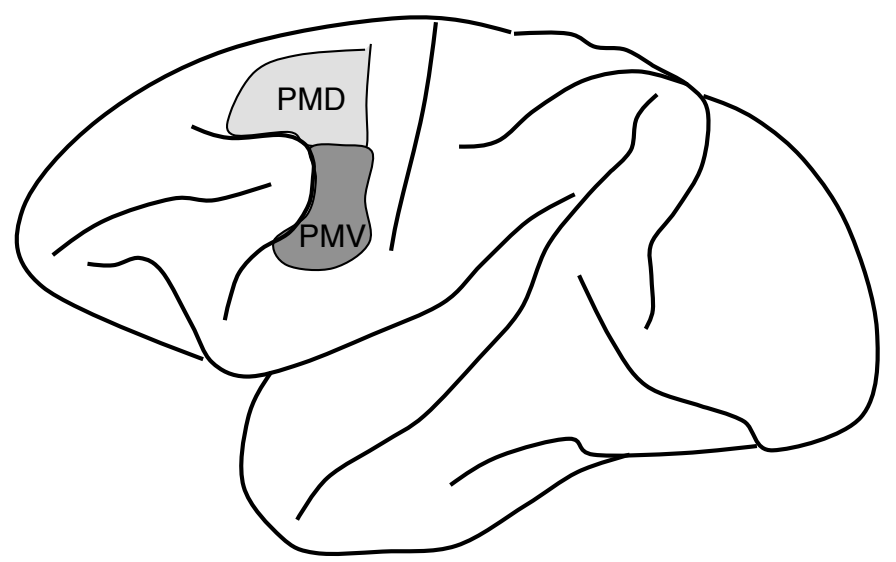

b

C
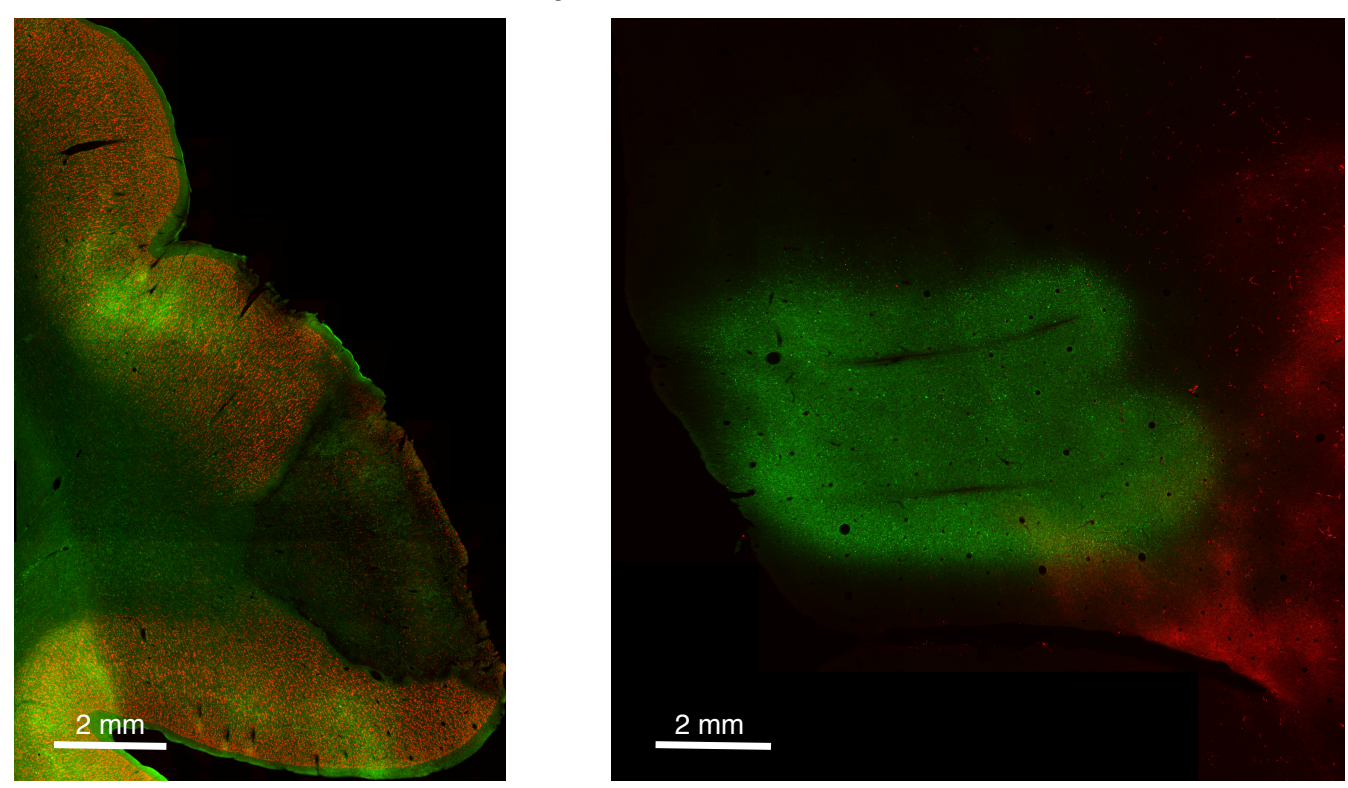

Figure 19: Immunofluorescence stainings of rAAV5 injected Macaca mulatta cortex (A) Schema of cortex regions PMV and PMD (B) Ventral pre-motor cortex injection site of rAAV2/5-CaMKII-eNpHR3.0-eYFP (C) Frontal eye field injection site of rAAV2/5-CaMKIIa-hChR2(H134R)-eYFP-WPRE. Slices were stained for NeuN (red) and eYFP (green). This experiment and micrograph B are partially as well described in Huer (2018). 


\section{2 $\mathrm{CD} 9_{\mathrm{GFP}}$ over-expression for a higher yield of exo-AAV1}

This section's aim was to elucidate the impact of an increased exosome release by CD $9_{\mathrm{GFP}}$ over-expression of rAAV producer cells on the yield and efficiency of exo-AAVs, which had been harvested from the cell culture media. As a proof of concept we demonstrated that during rAAV1 production the exosome and exo-AAV1 yield increased and additionally we gained more efficient exo-AAVs. Unlike rAAV1, which besides had also been used in the previously published studies on exo-AAVs (for details see 1.2.1), rAAV5 is not efficiently released in the media. Therefore we chose the first for this study.

CD9 is a well known exosomal marker. In a publication, in which I am contributing author, we recently demonstrated that its over-expression boosts the exosome production in several human cell lines and additionally increases the infectivity of lentiviruses (see supplemental 5.5 and Böker et al. (2017)).

To perform standard rAAV and exo-AAV production under boosted exosome conditions, HEK-AAV producer cells were transduced with lentiviruses (LV-CD $9_{\mathrm{GFP}}-\mathrm{VSV}-\mathrm{G}$ ) for $\mathrm{CD} 9_{\mathrm{GFP}}$ over-expression. The cells were tracked by flow cytometry analysis and considered useful for rAAV production when at least $80 \%$ of the cells were GFP positive. The $\mathrm{CD} 9_{\mathrm{GFP}}$ expression was also visible in fluorescence microscopy and predominantly localised in cell membranes.

The rAAVs were produced by the standard transfection procedure with pDP1rs and pAAV-CAG-GFP. Subsequently, standard rAAVs were harvested from the cell lysate as previously described, whereas exo-AAVs were harvested by sequential centrifugation steps of the media (Figure 20). 
a

rAAV1 production in HEK-AAV-CD9 $9_{\text {GFP }}$ cells Transduction
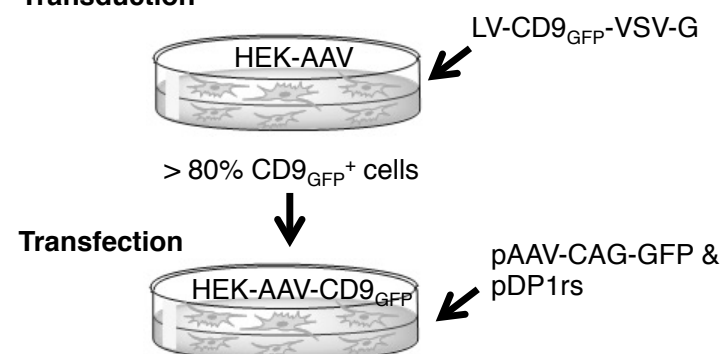

standard rAAV
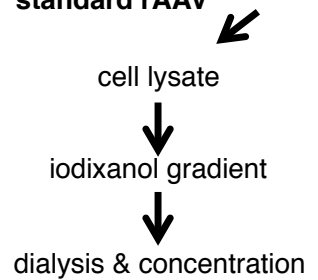

exo-AAV

media

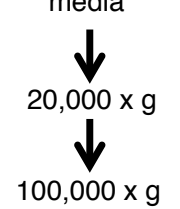

b

HEK-AAV

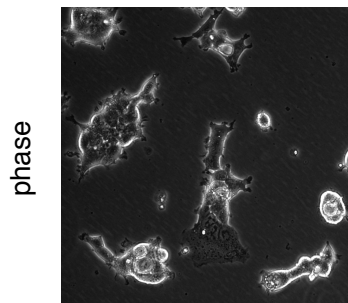

$\frac{0}{\frac{1}{0}}$
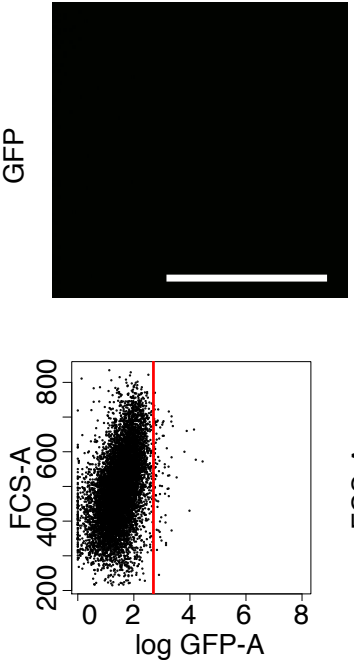
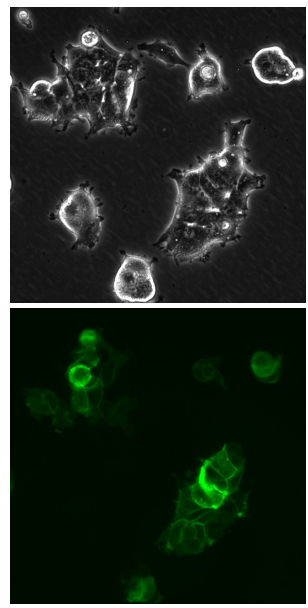

HEK-AAV-CD $9_{\text {GFP }}$

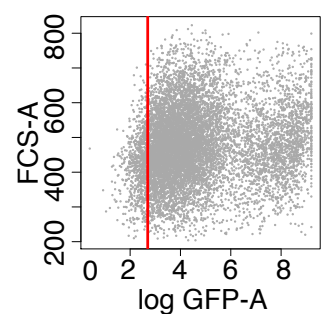

Figure 20: Overview of HEK-AAV-CD9 ${ }_{\mathrm{GFP}}$ cell production and standard and exoAAV harvest (A) HEK-AAV cells were transduced with LV-CD9 $9_{\mathrm{GFP}}-\mathrm{VSV}-\mathrm{G}$ to gain $\mathrm{CD} 9_{\mathrm{GFP}}$ over-expression. The transgene cells were used for rAAV production when at least $80 \%$ were determined to be $\mathrm{CD} 9_{\mathrm{GFP}}$ positive by flow cytometry analysis (B). rAAV production requires the transfection of the genetic construct and the helper plasmid (pDP1rs). The standard rAAVs were harvested from the cell lysate whereas exo-AAVs were harvested from the cell culture media via sequential centrifugation. Scale bar $200 \mu \mathrm{m}$. Creative commons license CC BY-NC-ND 4.0, modified from Schiller et al. (2018).

\subsubsection{CD9 $9_{\mathrm{GFP}}$ over-expression boosts exosome release in culture media}

Our first aim was to affirm whether recombinant CD9 $9_{\mathrm{GFP}}$ over-expressing HEK-AAV cells have an increased exosome secretion as we similarly published for five other human cell lines (see supplemental 5.5 and Böker et al. (2017)).

We firstly harvested vesicles of the media from wild type (HEK-AAV) and CD9 $9_{\text {GFP }}$ overexpressing cells via polyethylene glycol (PEG) precipitation and secondly via the exo-AAV purfication procedure. The concentration and size distribution of extracellular vesicles was determined by nanoparticle tracking analysis (NTA) (Figure 21). 
The over-expression led to a significantly increased amount of PEG-precipitated vesicles (1.96 times) with a mean size reduced from $210 \mathrm{~nm}$ to $184 \mathrm{~nm}$. Thereby, the exosome concentration (30-100 nm) in the media of over-expressing cells significantly increased 3.75 times as much.

Next, we elucidated whether the pellets of the exo-AAV harvesting procedure $(20 \mathrm{k}$ and $100 \mathrm{k}$ ) from wild type and over-expressing cells differed in the vesicle distribution. To have comparable cell physiological conditions, we mimicked the rAAV production conditions by the transfection of pAAV-CAG-GFP. The conditioned media was processed according to the exo-AAV harvesting protocol. Additionally, vesicles from the media and supernatant were harvested by PEG precipitation because the vesicle concentration for a direct analysis by NTA was too low. Being as reproducible as during PEG precipitation, the over-expressing cells had the similar trend in exosome concentration for all pellets. Especially the 100k pellet showed a higher proportional increase of exosomes with 5.75 times of the wild-type (Figure 21D). The profile of the vesicle size distribution in the 100k pellets showed enrichment of vesicles in exosome size, whereas the $20 \mathrm{k}$ pellets and the supernatants included more bigger vesicles up to over $600 \mathrm{~nm}$ (Figure 21E with exemplary profiles for HEK-AAV-CD9 $9_{\mathrm{GFP}}$ cells). 
We verified the abundance of exosomes and CD $9_{\mathrm{GFP}}$-positive exosomes for the transgene cells and rAAVs in the $20 \mathrm{k}$ and 100k pellets via Western Blot with antibodies against the AAV capsid proteins, GFP and the exosomal markers (hAlix, CD9, and CD63) (Supplemental Figure 36).

a
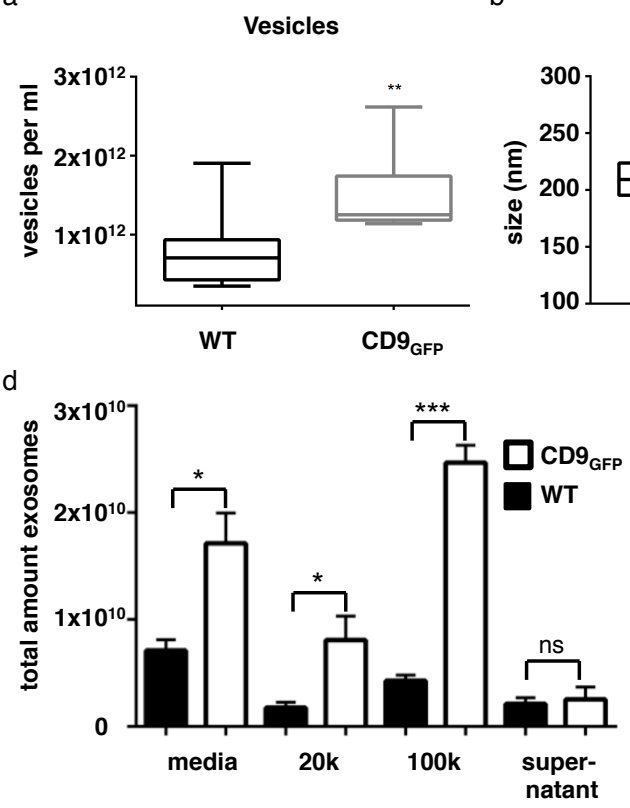

b

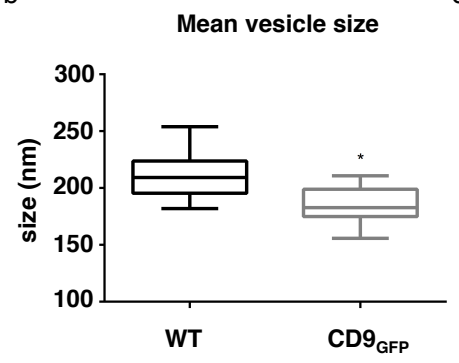

c

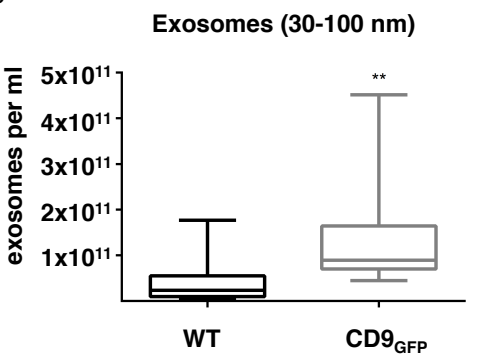

e

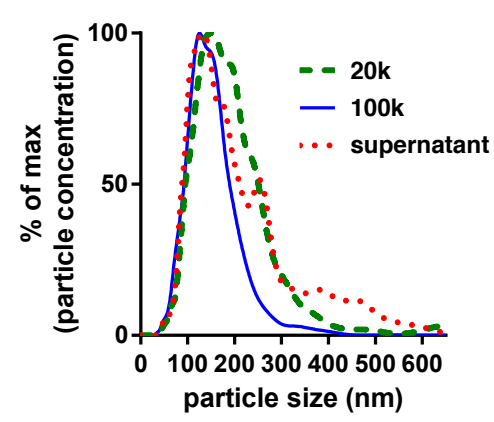

Figure 21: CD9 $9_{\text {GFP }}$ over-expression in HEK-AAV cells influences the vesicle content in the media and the samples during exo-AAV harvesting procedure NTA of PEG precipitated extracellular vesicles of $\mathrm{CD} 9_{\mathrm{GFP}}$ over-expressing HEK-AAV cell media showed a significantly increased amount of vesicles compared to wild type cells $(\mathbf{A})$, which were reduced in mean size $(\mathbf{B})$ and had an increased amount of vesicles in the size range of exosomes (30-100 nm) (C). (A, B and C) Samples of biological triplicates were measured in technical triplicates. Significance was tested via unpaired t-test for normally distributed data (B) and via Mann-Whitney test for non-normal distributed data $(\mathrm{A}$ and $\mathrm{C})$. Error bars indicate the standard deviation, ${ }^{*} \mathrm{P}<0.05,{ }^{* *} \mathrm{P}<0.01$. (D) The exosome amount during mimicked exoAAV harvesting procedure was in each step higher for the CD9 $9_{\text {GFP }}$ over-expressing cells. (E) Exemplary size distribution profile of vesicles in the $20 \mathrm{k} \mathrm{x}$ g pellet, $100 \mathrm{k}$ $\mathrm{x} g$ pellet, and remaining supernatant of $\mathrm{CD} 9_{\mathrm{GFP}}$ over-expressing cells. Creative commons license CC BY-NC-ND 4.0, modified from Schiller et al. (2018).

\subsubsection{CD9 $9_{\text {GFP }}$ over-expression yields more efficient and increased amounts of exo-AAVs}

Having set up the exo-AAV production in wild type and $\mathrm{CD} 9_{\mathrm{GFP}}$ over-expressing cells and having demonstrated that the latter had an increased number of exosomes in the 
$100 \mathrm{k} \mathrm{x}$ g pellet, we elucidated whether this increased release of exosomes causes a higher amount and/or more functional exo-AAVs as well. Therefore, we quantified the genomic titer by real-time PCR and the functional titer by flow cytometry analysis (Figure 22A). At first, we observed no significant difference in the genomic titers for both cell types in any of the purification steps (Figure 22B).

For determining the functional titer, we secondly applied serial dilutions of the exo-AAV1 media and 100k pellet on HEK cells and analysed them for GFP expression. We plotted the data of technical triplicates over the applied vector genomes (vg) and fitted a linear regression (Figure 22B and Supplementary Figure 36). The slopes from the regression lines for exo-AAVs and exo-AAV-CD9 $9_{\mathrm{GFP}}$ significantly differed, meaning that the exo-AAVs from the over-expression cells were more efficient. Besides, we disproved in a control experiment that the application of $\mathrm{CD} 9_{\mathrm{GFP}}$ exosomes alone leads to GFP positive HEK cells. 


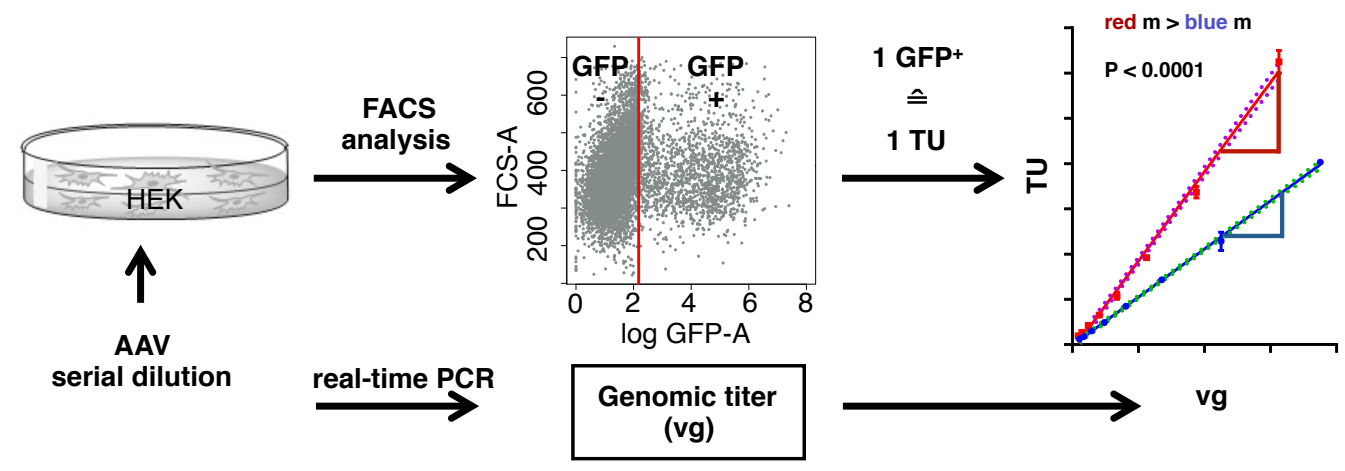

b

C
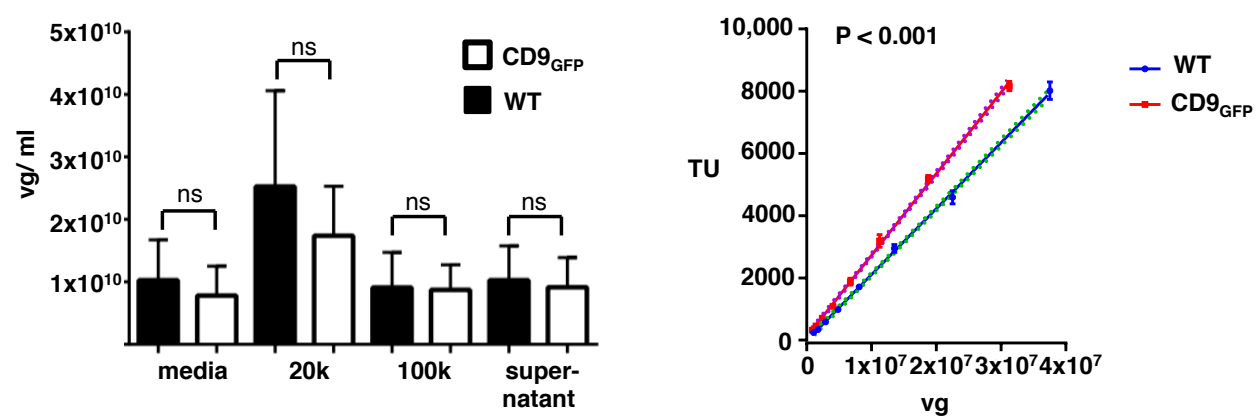

Figure 22: Enhanced transduction efficiency of exo-AAV1-CD9 ${ }_{\mathrm{GFP}}(\mathrm{A})$ Method diagram of rAAV titration. HEK cells were transduced with a dilution series of exo-AAV1 and exo-AAV1-CD9 $9_{\mathrm{GFP}}$ and the number of CD9 $9_{\mathrm{GFP}}$ expressing cells was determined by flow cytometry analysis. The transducing units are plotted over the applied vector genomes. Standard deviations of technical triplicates are shown by the error bars. Linear regression lines (red and blue) were fitted and the P-value (two-tailed) calculated to test whether the slopes are significantly different. (B) Genomic titer of three exo-AAV production rounds. Mean and error bars indicating the standard deviation. (C) An exemplary plot for 100k pellet exo-AAVs.Creative commons license CC BY-NC-ND 4.0, modified from Schiller et al. (2018).

The quotient of $\mathrm{vg} / \mathrm{TU}$ characterises the efficiency of a virus, with a lower quotient indicating a higher efficiency. Exo-AAV1-CD $9_{\mathrm{GFPS}}$ were $26 \% \pm 4.9 \%$ (mean $\pm \mathrm{SD}$ ) more efficient than the wild-type exo-AAV1s in independent biological triplicates. The media, which contains a mixed population of standard and exo-AAVs, showed a smaller but also significant boosting effect of around $7 \%$ (Table 44).

It is important to point out, that the same genomic titer of viruses derived from CD $9_{\mathrm{GFP}}$ over-expressing cells transduced a higher amount of cells. 
Table 44: Ratio of vector genomes per transducing units for 100k pellet and media

\begin{tabular}{cccc}
\hline Fraction & \multicolumn{2}{c}{$\begin{array}{c}\text { vg/TU } \\
\text { exo-AAV1 }\end{array}$} & $\begin{array}{l}\text { \% exo-AV-CD9 } \\
\text { efficiency }\end{array}$ \\
\hline exo-AAV1-CD9 & GFP & \\
100k pellet & 1131 & 838 & +26 \\
& 2894 & 1968 & +32 \\
Media & 4699 & 3743 & +20 \\
\hline
\end{tabular}

Having observed that exo-AAV1-CD $9_{\text {GFP }}$ was up to $32 \%$ more efficient, we wanted to elucidate whether this was the reason of a higher number of exosome-bound rAAVs due to the higher number of released exosomes. We used an iodixanol density gradient to separate vesicle-bound rAAVs from standard rAAVs.

Standard rAAV1, as well as exo-AAVs from wild-type and over-expressing cells, were subjected to the gradient, ranging from 6 to $60 \%$ iodixanol. After centrifugational separation, we harvested 18 fractions of $1 \mathrm{ml}$ (Figure 22A). The genomic titer of the fractions was quantified and plotted as the percentage of total genomic copies (Figure 22B). The standard vector served as our control to categorise the rAAVs together with the published distribution of exosomes and exo-AAVs in two fractions (György et al., 2014; Cantin et al., 2008). The standard rAAVs migrated to high-density fractions and exosome-bound rAAVs to low-density fractions (indicated in figure 22A).

As expected from previous findings (see Figure 8), we mainly recovered standard rAAV1 (98.5\%) in the high density fractions (14-18). Whereas exo-AAV1 migrated in low-density fractions (4-13) with $29.25 \%$, and exo-AAV1-CD9 $9_{\text {GFP }}$ with $47.66 \%$ which is around $39 \%$ more. For all samples a rather negligible less than $1 \%$ remained in the lowest-density fractions (1-3) and was not further evaluated. 
a

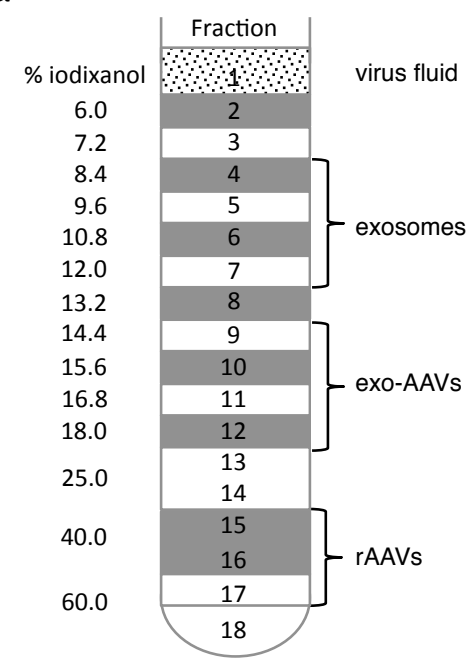

$\mathrm{b}$

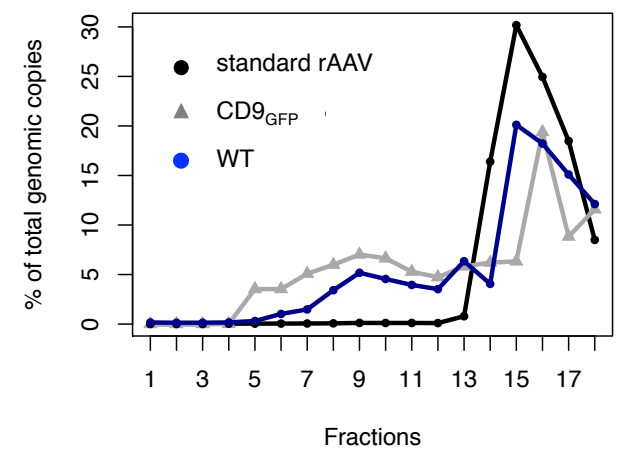

C

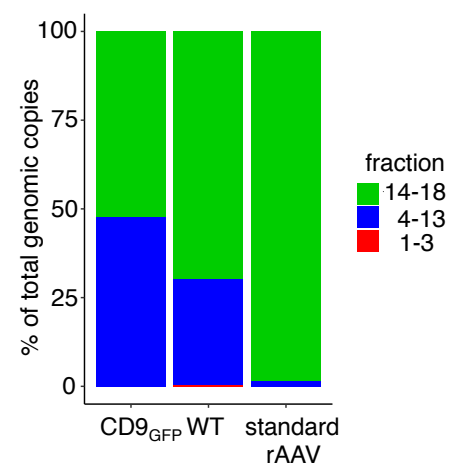

Figure 23: Exo-AAV1-CD9 ${ }_{\text {GFP }}$ enriched in lower-density fractions (A) Schema of the iodixanol gradient with indicated fractions to which exosomes (Cantin et al., 2008), exo-AAVs (György et al., 2014) and standard rAAVs are expected to migrate. (B) Standard rAAVs and exo-AAVs from wild type and CD9 $9_{\text {GFP }}$ over-expressing cell lines were loaded onto a gradient and fractions were analysed after centrifugation for vector genomes. (C) \% of total vector genomes categorised for fractions 1-3, 413, and 14-18. Creative commons license CC BY-NC-ND 4.0, modified from Schiller et al. (2018).

To sum up, we proved that $\mathrm{CD} 9_{\mathrm{GFP}}$ over-expressing HEK-AAV cells produced a higher amount of exosomes and we found out that exo-AAVs produced in these cells were $26 \% \pm$ $4.9 \%$ (mean $\pm \mathrm{SD}$ ) more efficient, compared to the production in wild type cells. Additionally, these exo-AAV1-CD9 $9_{\text {GFP }}$ samples contained $39 \%$ more exosome-bound AAVs. These results indicate that the higher transduction efficiency of exo-AAV1s from $\mathrm{CD} 9_{\mathrm{GFP}}$ overexpressing cells is due to the increased amount of rAAVs which are exosome-associated.

\subsubsection{Exo-AAV serotypes 1 and 6 transduce mesenchymal stem cells}

We further wanted to elucidate the performance of exo-AAVs from wild type and CD $9_{\mathrm{GFP}}$ over-expressing producer cells on primary cells. Therefore we applied the same number 
of genomic copies from exo-AAVs of serotype 1 and 6 on MSCs from the donors \#83 and \#97. We determined the percentage of the GFP transgene expressing cells (under the CAG promotor) by flow cytometry analysis one week after transduction.

The two donors had a different susceptibility for the exo-AAVs, whereas donor \#97 was more susceptible in general. Exo-AAVs of the serotype 6 led to a higher transduction than exo-AAVs of serotype 1. Exo-AAV6-CD9 $9_{\mathrm{GFP}}$ was slightly more efficient than exo-AAV6 on donor \#83, whereas the exo-AAVs from the wild type cell line transduced more cells in the other experiments. We observed no expression for exo-AAV1-CD9 ${ }_{\mathrm{GFP}}$ with donor \#97.

Taken together, the exo-AAVs had a different performance on MSCs, which depended on their serotype. And neither the $\mathrm{CD} 9_{\mathrm{GFP}}$ positive nor the $\mathrm{CD} 9_{\mathrm{GFP}}$ negative exo-AAVs had a generally higher transduction efficiency.

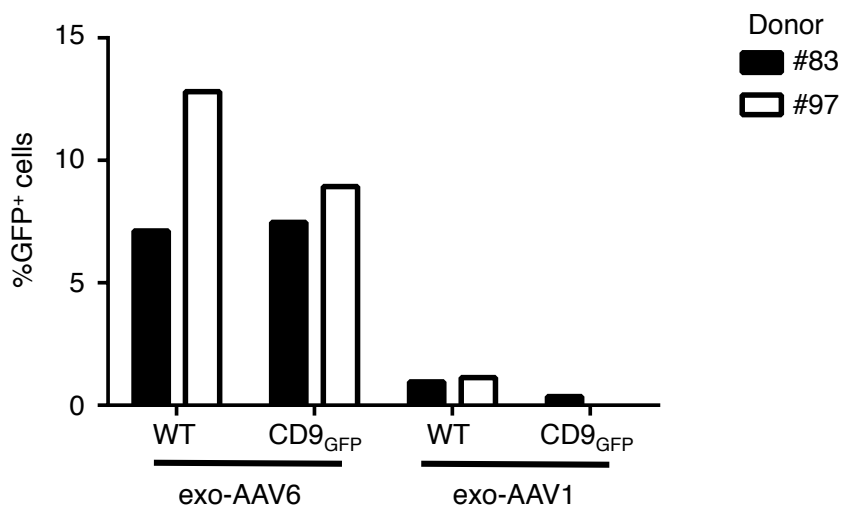

Figure 24: Exo-AAV and exo-AAV-CD9 $9_{\mathrm{GFP}}$ of serotypes 1 and 6 transduce mesenchymal stem cells Mesenchymal stem cells from donor \#83 and \#97 were transduced with exo-AAV and exo-AAV-CD9 $9_{\mathrm{GFP}}$ of serotypes 1 and 6 and the percentage of GFP positive cells was evaluated by flow cytometry analysis one week after transduction. 


\subsection{Lentiviruses as neuronal delivery tool}

As a short chapter in this study but for completion of the delivery tool platform, we generated replication deficient HIV-1-based lentiviruses. Analogous to the rAAVs and VLPs in this study (see 3.1 and 3.4.2), three different viruses were produced, transferring one of the commonly used promotors for neuronal delivery (CAG, CaMKII, and hSyn) and the fluorescent transgene GFP.

As recently published in a paper where I am contributing author, we first discovered the enhancing effect of CD9 on the secretion of exosomes and the infectivity of vectors during the production of lentiviruses (see supplemental 5.5 and Böker et al. (2017)). In combination with the rAAVs and exo-AAVs the here produced lentiviruses are a suitable tool for future investigations to clarify the mode of action of CD9 which still hasn't been unraveled yet.

We tested the three lentiviral preparations for their functionality. Therefore, we applied equal volumes of lentiviral samples on HeLa cells and took micrographs $72 \mathrm{~h}$ post transduction (Figure 25). The lentivirus with CAG and CaMKII promotor drove a strong GFP expression in a high number of cells, whereas hSyn only drove expression in a low number of cells.

The functionality of the lentiviruses was qualitatively proven. This allows comparing the tools' efficiency in upcoming approaches in combination with exo-AAVs and VLPs with or without retargeting in transduction of the target tissue.

Due to their faster and stronger expression, the lentiviruses can be used to investigate new opsin constructs in ex vivo experiments which require a quick expression onset. 

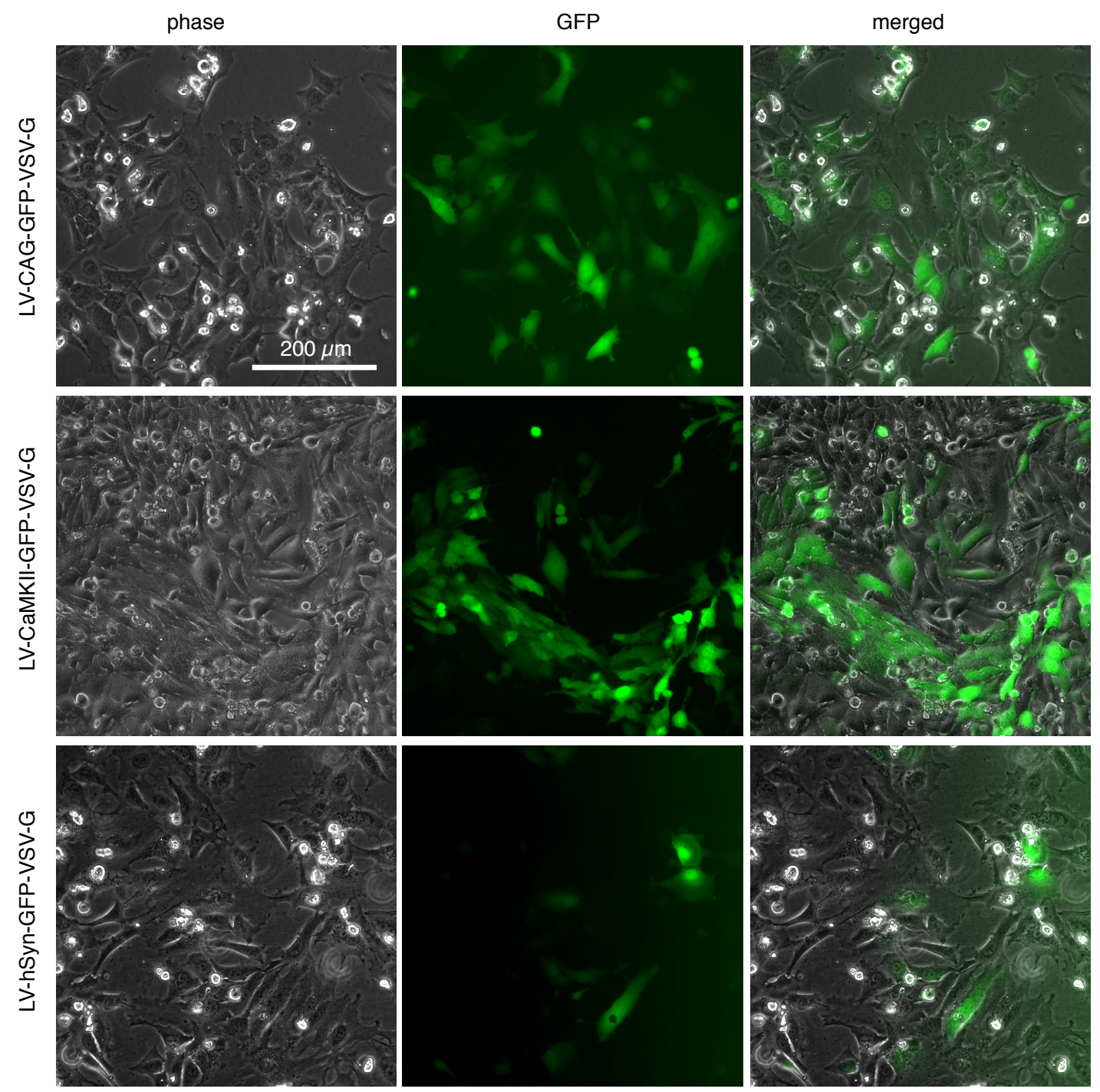

Figure 25: Lentiviruses deliver the transgene in HeLa cells Lentiviral constructs driving GFP expression under CAG, CaMKII and hSyn promotors were tested for functionality in HeLa cells. 


\subsection{Virus-like particles as delivery tool for neuronal targets}

In our group, the non-viral JC Polyoma virus-derived VLPs have mainly been used to deliver small RNAs in vitro and in vivo so far. The aim of this chapter was to develop DNA delivery strategies with enhanced safety which can potentially be applied to gene therapy and particularly to optogenetics. Moreover, we aimed a neurotrophic retargeting of the VLPs' tropism.

\subsubsection{Delivery of linear expression cassettes and MIDGE vectors}

Aiming to gain a space-saving and secure gene construct, the pGEM-T Easy CAG-GFPWPRE was generated. A linear expression construct of around $2.5 \mathrm{~kb}$ in size can be generated by enzymatic digestion with SphI and SacI. This was constructed analog to the control rAAVs and lentiviruses to allow an easy comparison of the three delivery tools (see 3.1 and 3.3). The linear construct lacking the bacterial plasmid backbone has the benefit of a reduced number of CpG-motifs, which are prone to cause an immune response (Krieg, 1999).

More importantly, to make the delivery of linear DNA with the VLPs even safer, the restriction sites were selected in a way to enable the generation of MIDGE vectors (Schakowski et al., 2001). The linear DNA was purified and oligonucleotides, forming a hairpin structure, were used to recircularize the DNA. Subsequently, all remaining oligonucleotides and non-circularized DNA were digested by the T7 exonuclease (Figure 26 A). As control for a complete digestion, linear DNA was incubated at the same conditions and aliquots of the samples were analysed on agarose gels (Figure $26 \mathrm{~B}$ ). We found that the MIDGE vector was resistant to T7 exonuclease digestion, whereas the linear DNA was completely digested. Furthermore, we could prove that no oligomerization had occurred, which would multiply the construct size. 
a
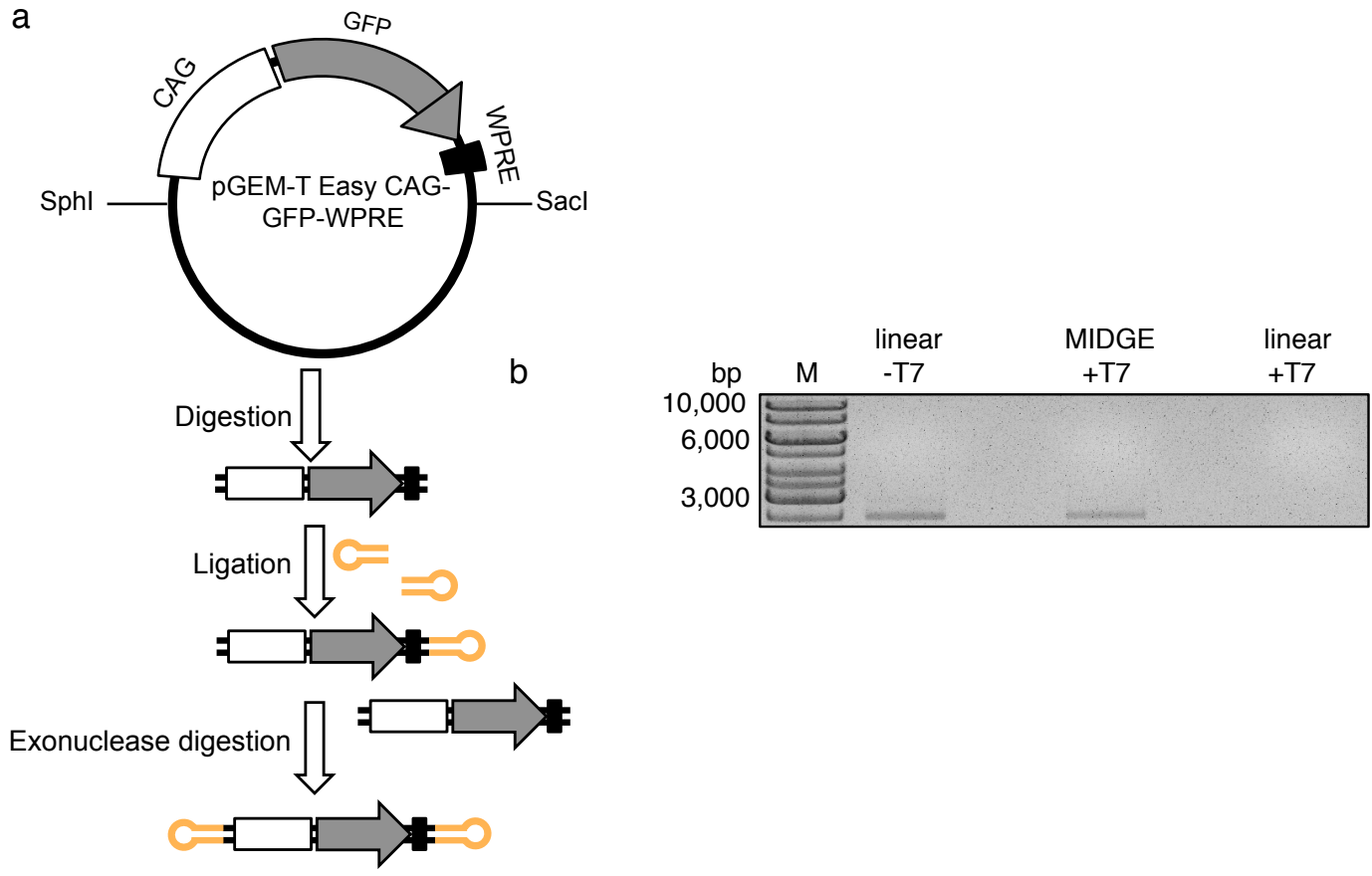

Figure 26: Production of MIDGE vectors (A) The MIDGE vector derived from an enzymatic digested plasmid. Its cohesive ends were connected with oligonucleotides. Remaining not-closed DNA was digested by a T7 exonuclease. (B) Agarose gel to prove successful T7 exonuclease digestion.

As a proof-of-concept, equal amounts of MIDGE vectors and the linear expression construct, packaged in VLPs, were applied on SKBR3 cells and the percentage of GFP positive cells was evaluated $48 \mathrm{~h}$ after transduction via flow cytometry analysis (Figure 27).

In repeated experiments we found that both constructs transduced the cells in the same range. 

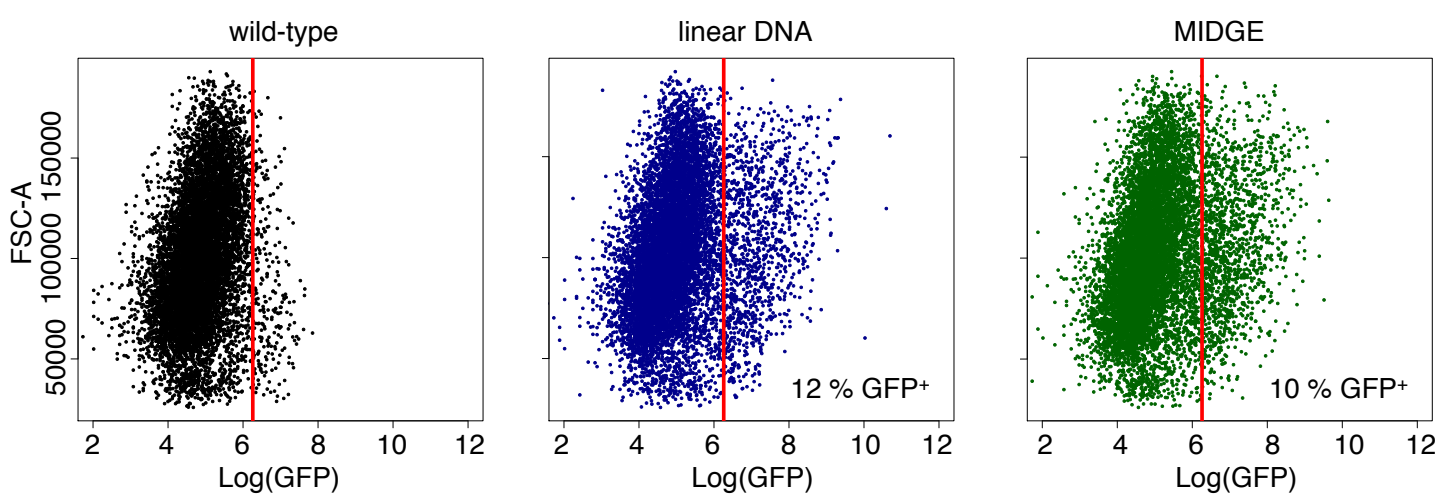

Figure 27: SKBR3 cells express linear and MIDGE vector after VLP delivery MIDGE vectors and linear DNA were packaged in VLPs and applied on SKBR3 cells. The red line in the dot plots indicates the $\log ($ GFP) value of two standarddeviations over the median fluorescence intensity of non-transduced cells. Cells above this value were considered as GFP positive. Whereat the percentage of cells above the cutoff-value from wild type cells was substracted.

\subsubsection{Virus-like particles for neuro-optogenetics}

For the use of the VLPs in neuro-optogenetics we evaluated whether linear expression cassettes with the CaMKII, and the hSyn promotor can be used to address a neuronal expression. We obtained expression of the transgene in HeLa cells with both promotors when transduced with rAAVs and lentiviruses (see 3.1 and 3.3). Therefore we then transduced HeLa cells with VLPs, harboring equal amounts of linear expression cassettes with the CAG, the CaMKII and the hSyn promotor and observed them for reporter gene expression.

But only cells transduced with the CAG promotor construct expressed GFP (data not shown).

As the transduction of HeLa cells with the CaMKII and the hSyn promotor lead to no expression, we evaluated their ability to drive expression in dissociated cortical cultures of the Wistar rat in the next step.

We found none of the constructs leading to a GFP expression visible in fluorescence microscopy. Moreover, cortical cultures transduced with cargo loaded or even empty VLPs died. The application of the reassembly buffer alone (in which the VLPs are suited after loading) did not affect the cell survival. And rebuffering of the VLP samples with the cell culture medium and pre-equilibration also caused cell death after transduction. A decrease of the VLP amount slowed down the cell death, but again, no GFP positive cells 
were detected (data not shown).

We concluded that our expression cassettes with neuron-specific promotors are not suitable to lead to a transgene expression in Hela cells when used as cargo for VLPs. Furthermore, cortical cultures of the Wistar rat react highly sensitive with cell death to the application of VLPs.

\section{Neurotrophic retargeting of virus-like particles}

We produced the VP1 protein which forms the self-organised VLP capsids in insect cell cultures and loaded the capsids with its cargo in vitro under ambient buffer conditions. The broad tropism of the JC polyoma virus-derived VLPs to $5-\mathrm{HT}_{2}$ receptor positive cells can be modified and adjusted on the course of biochemical manufacturing. Therefore, we express retargeting molecules, e.g. single chain variable fragments in E. coli or P. pastoris and chemically crosslink them via a maleimide-linker on the VLPs surface (Figure 28). Having one binding domain with each VP1 for the maleimide and the capsid consisting of 72 pentamers, one capsid has 360 potential binding-sites. Additionally, the VLPs' surface has a high affinity for carbohydrates, e.g., the naturally occurring $\operatorname{LST}_{\mathrm{C}}$. We found that biotin-coupled $\mathrm{LST}_{\mathrm{C}}$ is as well suitable to attach retargeting molecules to the VLPs surface when they, in turn, are expressed as streptavidin fusion protein.

Notably, the retargeting molecules can also be utilised to alter the tropism of exo-AAVs and lentiviruses when expressed and be located in the membrane of the producer cells. 


\section{Virus like particle production}

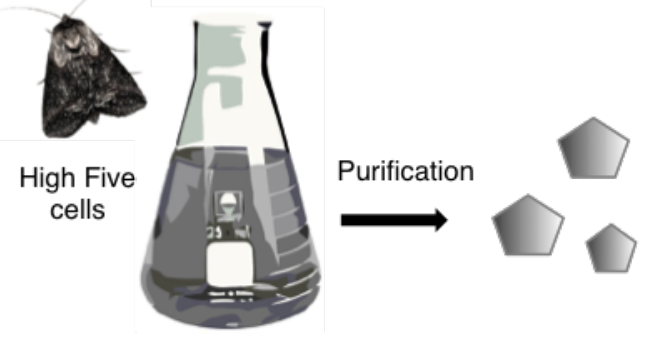

ScFv production

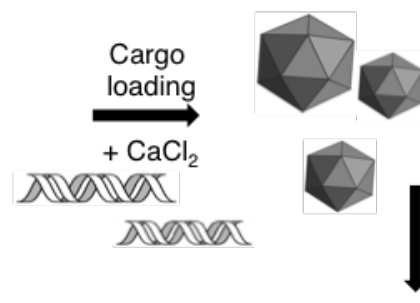

Cell transduction
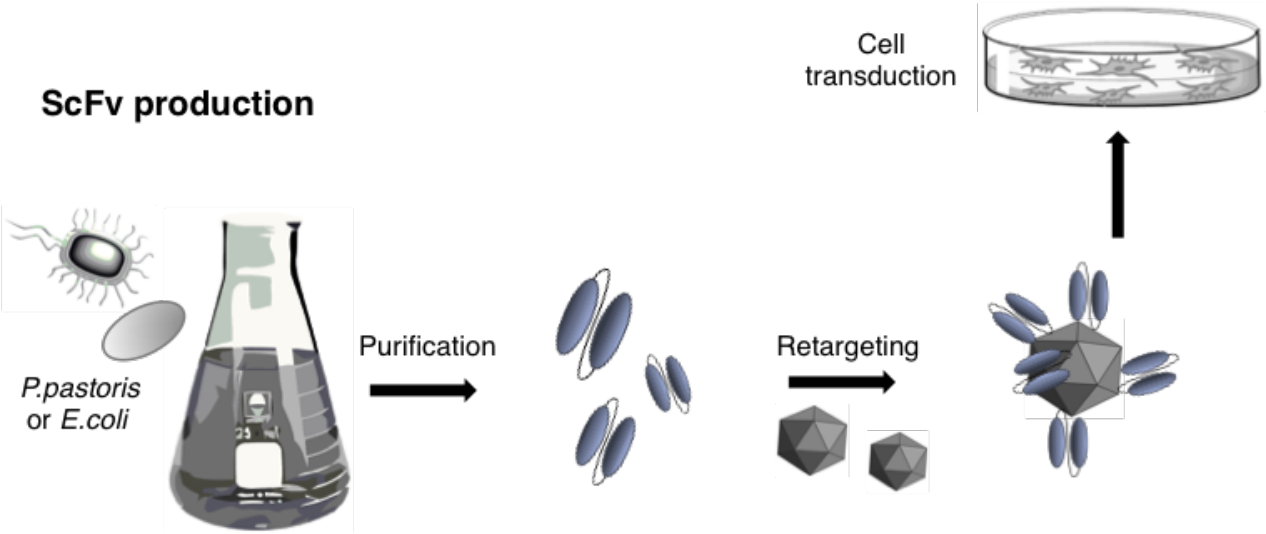

Figure 28: Production and retargeting of VLPs The VP1 protein is recombinantly expressed in insect cells. The purified VP1 protein reassembles to capsids in abundance of DNA and $\mathrm{CaCl}_{2}$, incorporating the cargo DNA. Retargeting molecules can be expressied in E. coli or P. pastoris and be attached to the VLPs surface. The native and the retargeted VLPs can be directly applied on cells.

After we had demonstrated that we could direct the delivery of the linear expression cassette to human cancer cells by switching the VLPs' tropism towards the Her2/neu, a growth hormone receptor frequently overexpressed particularly on breast and colon cancer cells (Experiments and Figure 29 were kindly provided by Stefan Schneider, German Primate Center, Göttingen), we aimed for a retargeting to neuronal cells. 

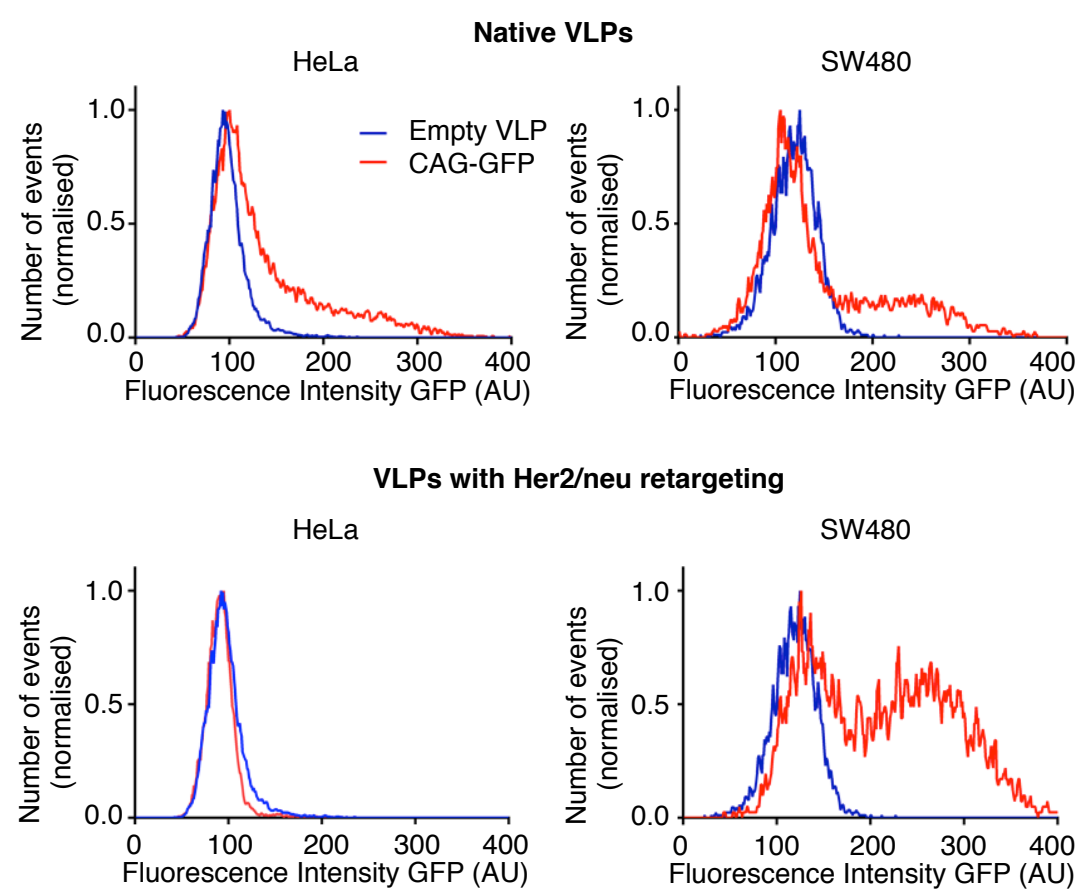

Figure 29: VLP retargeting to Her2/neu positive cells Flow cytometry analysis of HeLa and SW40 colon rectal cancer cell lines transduced with the CAG-GFP expression cassette. Native VLPs transduced $25 \%$ of both cell lines. Her $2 /$ neu scFv retargeted VLPs did not transduce HeLa cells anymore but $50 \%$ of SW480 cells. This figure was kindly provided by Stefan Schneider (German Primate Center, Göttingen).

We decided on a scFv that binds the receptor tyrosine kinase (TrkB) which is a neurotrophic factor receptor expressed in the central and peripheral nervous system and other tissues (Gupta et al., 2013). Due to its humanized glycosylation patterns, we preferred to express scFvs in the humanized P. pastoris. The expression constructs pPICZ $\alpha \mathrm{A}-$ TrkBscFv and pPICZ $\alpha$ A-TrkBscFv-Streptavidin were designed with an N-terminal cysteine for crosslinking and a C-terminal 6xHis-tag for FPLC purification and Western Blot detection. The plasmids were transformed into P. pastoris, and selection marker resistant clones were analysed for expression. Based on western blot detection, we found that none of the screened clones expressed the retargeting molecules.

Due to this result, the expression system was changed to E.coli (SoluBL21 ${ }^{\circledR}$ and BL21 $\left.\operatorname{Star}^{\circledR}(\mathrm{DE} 3)\right)$ and the pET28a(+) expression vector system. Various incubation temperatures (room temperature and $37^{\circ} \mathrm{C}$ ), incubation times and IPTG concentrations $(0.1 \mathrm{mM}$ up to $1 \mathrm{mM}$ ) for expression induction were tested.

The bacteria were harvested, lysed and the protein abundance in the soluble and nonsoluble fractions was detected via western blot. The two retargeting proteins (TrkBscFv 
$32 \mathrm{kDa}$ and TrkBscFv-Streptavidin $45 \mathrm{kDa}$ ) were well expressed under high IPTG condition, but stayed unsoluble under all tested conditions. Furthermore, several bands of higher and lower weight were visible for the TrkBscFv-Streptavidin construct.

As proteins for FPLC purification need to be soluble we could not pursue the retargeting approach with the TrkBscFv.
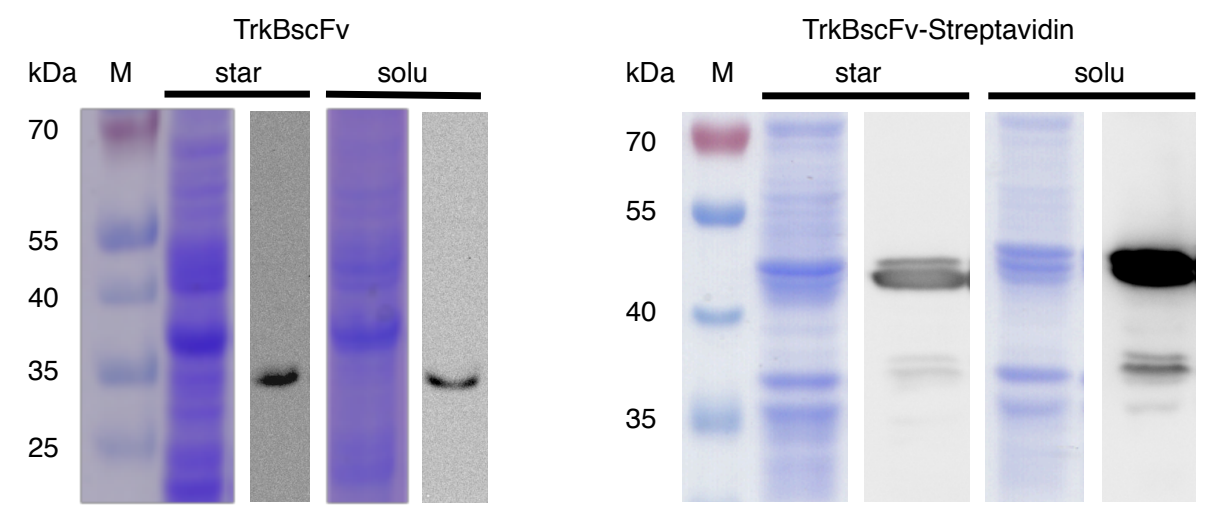

Figure 30: TrkBscFvs were expressed in E. coli but were only found in the unsoluble fraction Coomassie blue stained SDS-PAGEs and western blots of the whole cell lysate from SoluBL21 ${ }^{\circledR}$ (solu) and BL21 Star ${ }^{\circledR}$ (star) expressing the $31 \mathrm{kDa}$ TrkBscFv and $45 \mathrm{kDa}$ TrkBscFv-Streptavidin.

In 2016, Javed et al. published the use of an N-terminal truncated version of the rabies virus glycoprotein (RVG), which binds to the n-Acetylcholine receptor (nAchR), and thereby delivered a cargo siRNA to the CNS.

We added a C-terminal Gly-Ser-Gly-Ser-Cys sequence to the six nucleotides truncated version (RVGN6) to enable its chemical crosslinking on the VLPs.

For the retargeting, we loaded the VLPs with the linear expression cassette (see 3.4.1), attached the maleimide-crosslinker and incubated them together with the reduced RVGN6 in $30 \mathrm{X}$ excess. As controls, we used the loaded, but native VLPs to determine the percentage of transduction with the native tropism to the $5-\mathrm{HT}_{2}$ receptor. Only the peptide incubated with the DNA was used as negative control and to disprove that the peptide itself enabled delivery of the DNA. We only attached the crosslinker to the capsid which should completely mask the tropism of the VLPs. The native VLPs without crosslinker but only the peptide were used to elucidate whether the unbound RVGN6 would promote the transduction of $n A c h R$ positive cells.

We transduced three cell lines (SKBR3 and 3T3 are nAchR negative, SH-SY5Y is nAchR positive) with the five samples and evaluated the percentage of GFP positive cells five 
days after transduction. Due to the lack of a cell line, that is not transduced by the native VLPs but expresses nAchR, we used the neuroblastoma derived SH-SY5Y cell line. This should be transduced by both, the native VLPs and also by successfully retargeted ones. The murine fibroblast cell line 3T3 lacks the nAchR and should not be transduced after a successful retargeting (Lentz, 1990; Javed et al., 2016; Kovalevich and Langford, 2013). The native VLPs and VLPs incubated with the peptide alone transduced all three cell lines with at least $30 \%$ GFP positive cells up to over $70 \%$ for SH-SY5Y cells. No cells were transduced with our negative control. More interestingly though, both, the VLPs with attached crosslinker alone and the VLPs incubated with crosslinker and RVGN6 transduced less than $10 \%$ of the three cell lines.

To sum up, we assume that the crosslinker was attached to the capsid because the native VLPs transduced all cell lines but their transduction efficiency was highly reduced after the incubation with the crosslinker. Therefore we conclude that the reduced transduction efficiency of crosslinker and RVGN6 incubated VLPs can either be caused by a missing attachment of the retargeting peptide or the attached peptide prevents the binding to the cells.

Alltogether, VLPs are a powerful and easy to handle delivery tool. Furthermore, in combination with the MIDGE vector strategy and its possible alteration of tropism, they are also a promising tool for gene therapy. However, its ex vivo application and retargeting to nervous tissue requires further development. 
a

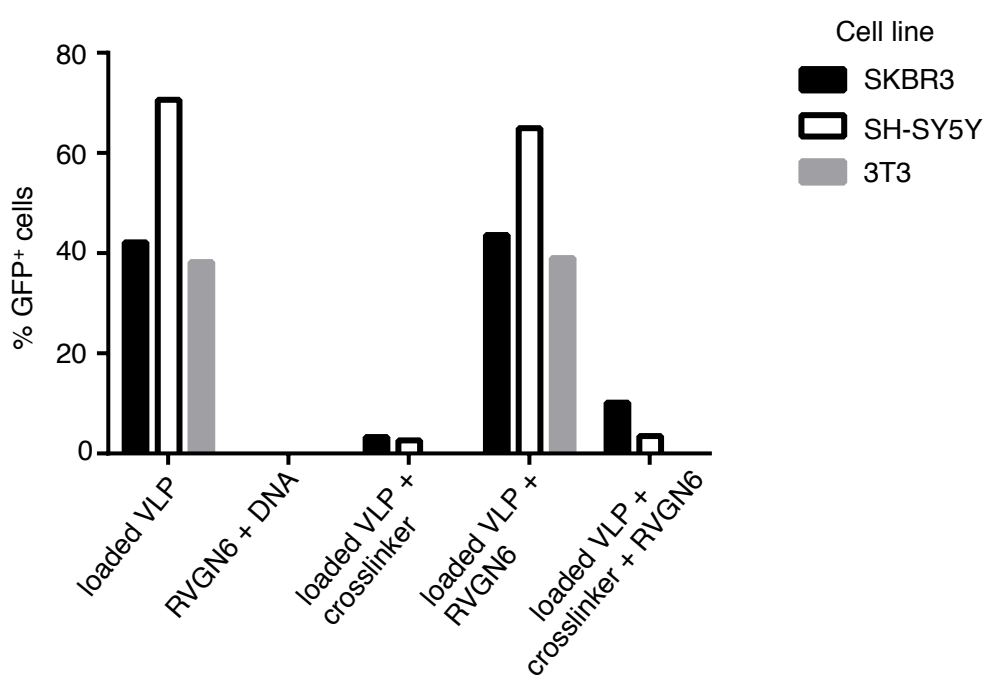

b

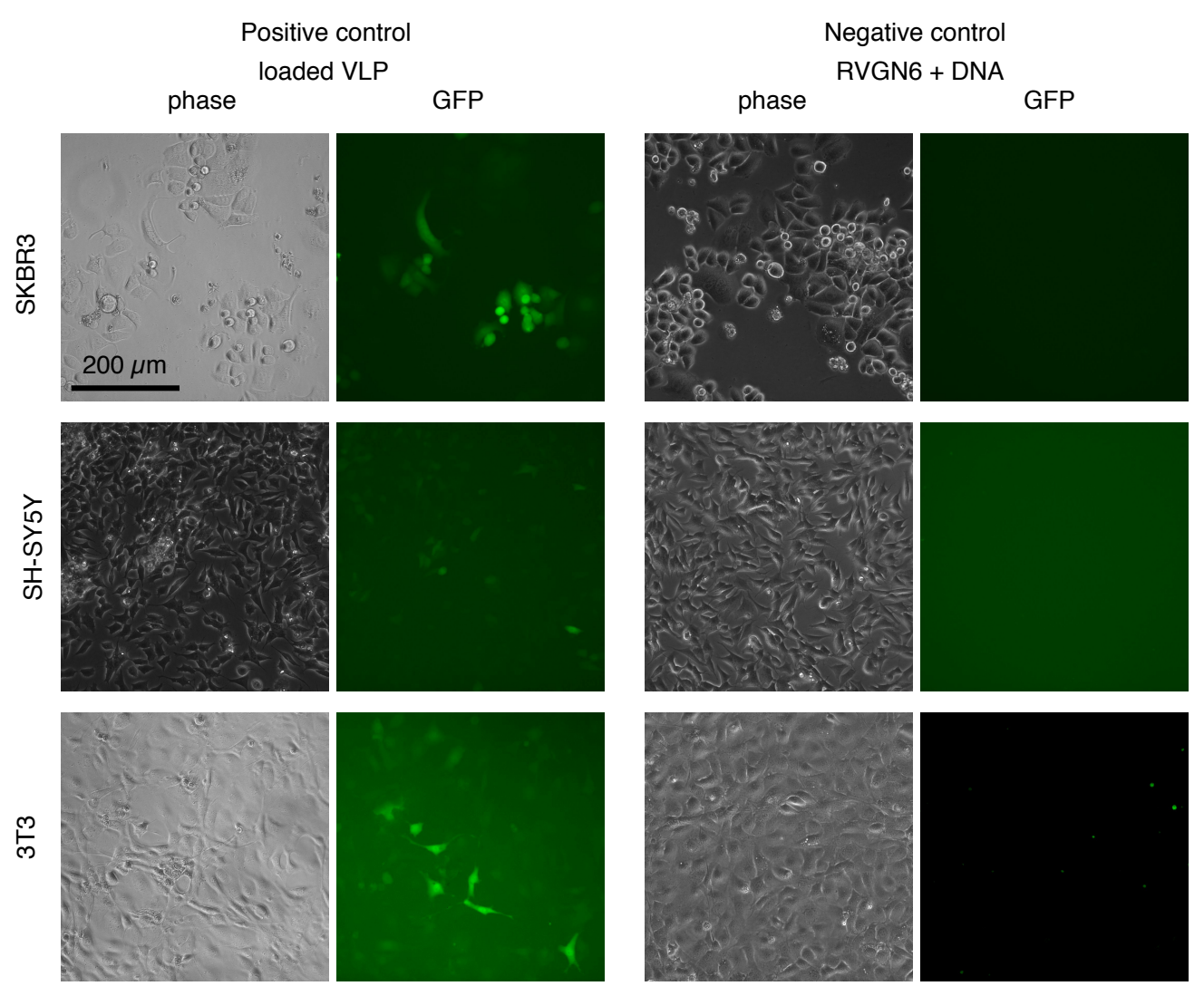

Figure 31: RVGN6 retargeting of VLPs (A) Transduction efficency of RVGN6 retargeted VLPs and controls was determined via flow cytometry analysis as percentage of transduced cells on day 5 after transduction. (B) Micrographs of SKBR3, SHSY5Y and 3T3 cells transduced with native VLPs as positive control and RVGN6 and the DNA alone as negative control. 


\section{Discussion}

Gene therapy projects mainly focus on the target identification or the constructs for specific research and therapy purposes. Others focus on engineering the delivery tools which are suitable for several applications. This thesis contributes to the latter. The main goals are to engineer viral and non-viral delivery tools for the gene transfer to nervous tissue. And an enhanced production of exo-AAVs and a neurotropic retargeting of VLPs. By that, we provide a toolbox to enhance the safety and efficiency of gene therapy.

A recently approved gene therapy for macula degeneration has been launched into the market with costs of approximately 450,000 US dollar per eye. Glybera in Europe even had to be taken from the market due to unachievable prices which made it unprofitable for the company to pursue its commercialisation.

The optimization of the tools and its production is a crucial element to reduce the costs of gene therapy. I hope that my work will help to make gene therapy achievable for a broader audience that would not be able to afford the high costs but then would benefit from current and future therapies.

\subsection{Standard rAAV production and evaluation}

\subsubsection{Standard rAAV production and evaluation on human cell lines}

With the rationale to engineer the exo-AAV production and purify rAAVs to in vivo grade for the use in non-human primates we implemented the standard rAAV production.

We successfully set up the production of five serotypes $(1,1 / 2,2,5$, and 6$)$ which enables investigators to choose the best-suited serotype for their future projects. Reports on the transgene delivery to neurons in the non-human primate brain utilise rAAV5 (Taymans et al., 2007; Gerits et al., 2012; Diester et al., 2011). In a cooperation project with such a group, we decided to implement the purification based on the serotype 5 . Of the 12 known serotypes, AAV5 is the most divergent one and is described to be complicated to purify and inefficient to apply in vitro (Janovitz et al., 2014). We concluded that a successful purification and ex vivo test systems should enable a high standard production 
of the other serotypes and their pre-animal test as well.

During their production, rAAVs are found in the cells and the medium. According to Vandenberghe et al. (2010), rAAV5 mainly remains in the cells and therefore our harvesting protocol uses the crude cell lysate. However, to increase the yield of other serotypes which are released from the cells, one should also harvest the rAAVs from the media. This can be performed by, e.g., PEG-precipitation (Arden and Metzger, 2016), tangential flow filtration (Clément and Grieger, 2016) or ultracentrifugation, as done for exo-AAV harvest (see 3.2).

Centrifugation-cleared crude lysate as a screening tool - With the rationale to provide several serotypes, we produced crude lysates of five commonly utilized serotypes and applied equal volumes of workflow preparations on five human cell lines to evaluate their transduction profile (Figure 6). The centrifugation-cleared crude lysate is the first applicable rAAV sample with the least required hands-on time and minimal equipment compared to more complex purification protocols. Within this study, the transduction efficiency was compared by the $\%$ of GFP expressing cells, without the inclusion of the expression level. We chose GFP as reporter gene because this fluorophor enables the identification of expression by simple fluorescence microscopy and by flow cytometry, both on a single cell level. Within neuro-optogenetics it is crucial to achieve a sufficient expression of the opsins in every single cell, essential to manipulate the cells electrical potential. In contrast, luciferase which is used in several studies only allows the quantification of the whole cell population protein expression (György et al., 2014). It is also more applicable for research projects that later aim to express, e.g., secreted lysosomal enzymes to treat storage diseases (Janson et al., 2002).

More interestingly, our data are in accordance with the study of Ellis et al., who published the comparison of transduction efficiencies of several rAAV serotypes in 2013. Different to our study, they applied equal numbers of viruses. Considering both studies, we can conclude that our production gained the same number of viruses for all serotypes.

Our findings provide the ability to choose the best transducible cell line in case that a serotype should be evaluated, or to select the right serotype for the desired cell line that should be transduced. In general, rAAV2 with the constitutive CAG promotor was most efficient on all cell lines. With the HeLa cell line being superior susceptible for almost all serotypes and HEK cells for rAAV1, we subsequently used them for the quantification of the functional titer (see 3.1.1 and 3.2.2).

Production of a good quality virus - The two good quality features of a viral sample are their high functional titer and high purity. We therefore looked for a protocol, yielding a 
high titer and pure sample and compared our preparations with reference viruses which are used in several publications (see 2.3.7) (Zhang et al., 2010; Gerits et al., 2012; Diester et al., 2011). The output of the genomic titer can be the same for different purification protocols, but the purification can influence the bioactivity of an apparently intact particle as, e.g., longer exposure to cesium chloride minimizes the bioreactivity (Hermens et al., 1999). An interesting, but not yet applicable finding under GMP conditions is that cellular contaminants can enhance the vectors' transduction efficiency (Tenenbaum et al., 1999).

Centrifugation purification and concentration - As a fast and low equipment-requiring protocol, we first elucidated the purification and enrichment effect of a 21,000X g centrifugation which other groups use for virus purification for in vivo experiments in rodents (personal communication with Carola Gregor, Max-Planck-Institute for Biophysical Chemistry, Göttingen).

However, we only detected a two-fold enrichment of the virus and a minor decrease of non-viral proteins which in conclusion did not reach our expectations of a high titer and high purity preparation.

Density gradient based purification - Next, we performed a density gradient centrifugation to more efficiently enrich the viruses and to separate them from cellular proteins. We chose this gradient based on the direct comparison of cesium chloride- and iodixanolbased purification by Strobel et al. (2015) who determined a higher purity of iodixanol gradient preparations. Besides the depletion of co-purified cellular proteins, the iodixanol gradient allows the depletion of empty AAV capsids. These by-products contain no viral genome, but they increase the amount of applied immunological targets. As an induced immune response can limit the transduction efficiency and prevent a repeated injection of the same serotype, it is essential to gain a high percentage of filled capsids. Additionally, the iodixanol density gradient is applicable for other serotypes and has the advantage over a cesium chloride gradient that iodixanol is inert in animals (Larsen et al., 1995).

For a laboratory-internal identification of the fractions with a high virus content we determined the genomic and functional titer of each gradient fraction. In the next step, to identify pure fractions, which only contained the virus, single-fractions were dialysed, concentrated and proteins were visualised on a silver-stained SDS-PAGE. Based on the visual bands the purity of fractions 13-18 (which also contained a high amount of the functional viruses) was confirmed, as impurities were visualised upon the upper fractions. To remove the iodixanol and thereby decrease the viscosity of the sample and to concentrate the virus in following preparations, the fractions 13-18 were pooled, dialysed and 
concentrated, increasing the titer up to $10^{11} \mathrm{vg} / \mathrm{ml}$.

$r A A V$ column purification - As an alternative or additional purification method, we elucidated FPLC based protocols with several columns. It is important to know that each serotype requires a specific equipment and protocol. However, none of the performed column and buffer combinations successfully bound the virus. Thus setting up FPLC protocols requires a detectable amount of the vector we cannot exclude that the total amount of applied virus was too low when comparing the applied viral load with a recent publication (Nass et al., 2017). This reflects once again that the rAAV purification requires a specialisation of the laboratory for rAAV production and even published protocols cannot right away be applied.

\subsubsection{Quality control and comparison with a reference virus}

Having set up a suitable purification protocol in our laboratory, we systematically compared our rAAV-sample quality with commercial reference viruses. We now provide a pipeline for rAAV production, purification and quality control which is further described and summarized in Figure 32. 


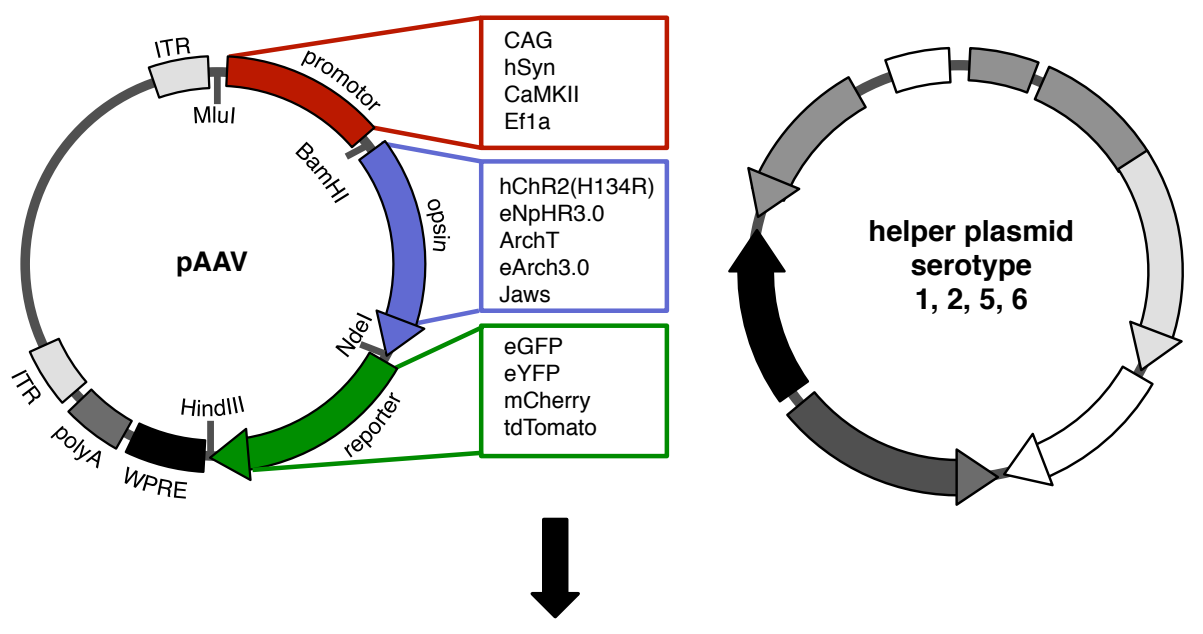

producer cell line

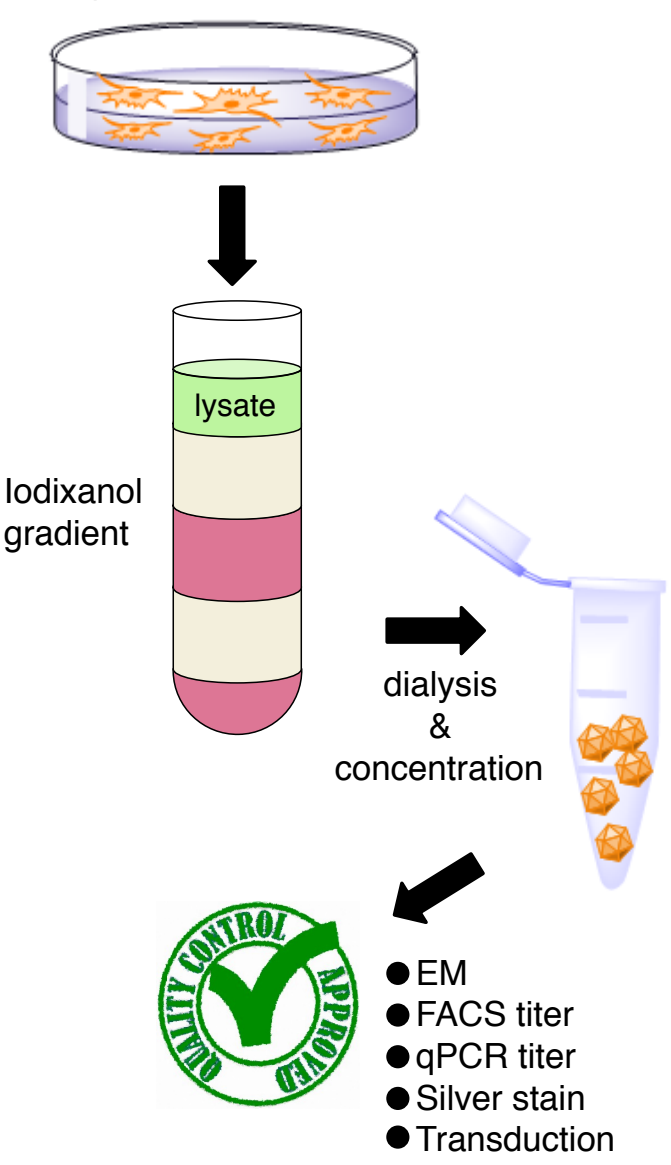

Figure 32: Workflow of rAAV production, purification and quality control With a plasmid from the rAAV genetic toolbox and a helper plasmid, determining the serotype, one of 800 different rAAVs can be produced. Double transfection of plasmids in HEK-AAV cells leads to formation of rAAVs within the cell. rAAVs in the crude cell lysate are purified from cell debris and empty capsids with an iodixanol density gradient. Fractions with pure and genome-containing capsids are dialysed and concentrated for a high titer preparation. Its quality is checked and determined by its purity (Electron microscopy (EM) and silver stain), high genomic titer (qPCR) and functionality (flow cytometry titer and transduction in vitro and ex vivo). 
Genomic and functional titer - Routinely, the genomic titer of a rAAV sample is declared, and a high titer is in the range of $10^{12}$ to $10^{13} \mathrm{vg} / \mathrm{ml}$. We set up a real-time PCR based quantification of the genomic titer with a set of primers binding within the WPRE sequence. This protocol can be applied for most of the rAAV plasmids and also for the quantification of the used expression construct for VLPs (see 3.1.2 and 3.4.1).

The quantification results can differ depending on the amplified sequence, as published by Wang et al. (2013a). To circumvent this bias, we quantified three reference viruses and found that our measurements were on average two-magnitudes lower as the declared titer $\left(10^{12}\right.$ versus $\left.10^{10} \mathrm{vg} / \mathrm{ml}\right)$. Besides the measurement bias, the storage and thawing cycles can reduce the titer, even if rAAV5 is hugely stable at short-term incubation at high temperature (Bennett et al., 2017). Compared with the reference, our self-produced virus has consequently a one magnitude higher genomic titer.

Most important is the functional titer, i.e., the concentration of transducing units, because it determines the transduction and therapy success. The reference virus approximately contained one magnitude more transducing units. In contrast to the CAG promotor driven GFP expression of our virus, the reference virus harbored the CBA promotor and no WPRE sequence. The chicken beta-actin (CBA) promotor and its derivative CAG are both constitutive promotors that drive a comparable long-term and robust transgene expression (Gray et al., 2011; Powell et al., 2015). As we quantified the functional titer by the number of GFP expressing cells, neglecting the amount of expressed protein, it was a suitable virus to compare with.

Comparing the ratio of the genomic and transducing titer ( $\mathrm{vg} / \mathrm{TU})$ enables the direct comparison of two viral samples with different genomic titers. A lower ratio determines a higher functionality. With the utilisation of the given titer, this ratio is lower for the selfproduced virus. Assuming a two magnitudes lower genomic titer of the reference virus, this slightly inverses the result.

Summing up, we achieved the production of a high titer and functional virus in comparison to the reference virus.

Sample purity - As mentioned, the second good quality feature of a virus sample is its purity. Meaning that it only contains the three viral capsid proteins and as little as possible empty capsids. Upon administration in vivo, the capsid load should be kept as low as possible to avoid an immune response and the empty capsids represent a non-useful by-product.

First, we separated the proteins of our virus sample on an SDS-PAGE and visualised them in high resolution with a silver stain. The reference virus contained only the three 
expected capsid protein bands, whereas in one of our productions an additional band with approximately $20 \mathrm{kDa}$ was visible. The second sample even contained a higher amount of co-purified proteins. This raises the question why the second virus contained more copurified proteins. It could be explained like this: firstly, the amount of applied crude lysate on the iodixanol gradient was higher for the second virus and thereby the cellular proteins might have smeared in the lower fraction. And secondly, due to the manual fraction-wise harvest of iodixanol gradients from top to bottom, proteins might have smeared in the lower fractions. To circumvent this issue, we consider to keep the volume of applied crude lysate rather low and to change the harvest procedure to a syringe-supported bottom-to top handling.

Electron microscopy with negative-stained rAAV samples offers the possibility to visualize the capsids and to assess the percentage of full particles (Zeltner et al., 2010). Our preparation protocol contains manual handling steps in the second range and thereby allows no quantitive titer comparison. However, we determined the percentage of filled particles and found approximately $40 \%$ for the reference virus as well as for the two own samples. We thereby fulfilled the quality criteria of the number of filled capsids.

However, others report vector preparations with over $90 \%$ filled capsids. Grieger et al. (2016) achieved this high percentage by a subsequent ion exchange chromatography which uses the subtle difference in charge of filled and empty capsids. Even if our production fulfills the reference criteria, it might be a future approach to decrease the number of empty capsids and further increase the quality of our preparation.

Additionally to the viral capsids, we found donut-shaped particles of around 10 microns in diameter in both of our preparations. To identify this co-purified protein, we analysed the corresponding SDS-PAGE gel area by mass spectrometry and observed ferritin light and heavy chain, keratin, and trypsin as main hits.

In agreement with the detection of the $20 \mathrm{kDa}$ protein in the SDS-PAGE and observations of Strobel et al. (2015), we identified the co-purified protein as ferritin, derived from the producer cell line. This is supported by the hypothesis that AAVs interact with ferritin during its life cycle. Trypsin and keratin are well-known bias in mass spectrometry. Prior to mass spectrometry the samples are treated with trypsin to fragment the proteins and keratins are arising during the sample processing from the experimenters (Link et al., 1999). Additionally, trypsin and keratin did not fit in the molecular mass profile of the excised gel area (20 kDa vs. approximately $60 \mathrm{kDa}$ ). Again, an adjustment during ion exchange chromatography was shown to separate rAAVs from ferritin (Grieger et al., 2016). The potential immunogenicity or toxicity of ferritin as an adjuvant in the in vivo application of rAAVs remains elusive because literature lacks studies on this subject to 
our knowledge. The application of both of our viral batches in the mouse cochlea showed none of those effects (see 3.1.3).

Genetic toolbox for straightforward expression construct generation - To further simplify the generation of a variety of rAAVs, we generated a genetic toolbox comprehending various promotors, opsins and reporter genes with optimised restriction sites. Thereby 80 combinations in straightforward and quick clonation steps are feasible and more importantly, also the easy inclusion of further genes. In addition to the rAAV production, this simplifies the production of lentiviruses and expression cassettes for VLPs as well. We provide a versatile, powerful tool that facilitates quick vector production for upcoming research demands.

Functional opsin expression - As proof of principle and to complete the rAAV pipeline, we confirmed that our virus drives a functional opsin expression allowing the generation of an illumination evoked photocurrent. HeLa cells can be used for the whole pipeline up to the voltage clamp recording.

To sum up, we implemented a pipeline for rAAV production and quality control with a genetic toolbox that enables vector production (rAAVs, lentiviruses and VLPs) in a versatile and experiment-demanding way (Summarized in Figure 32). We provide an expression study of various serotypes, promotors, and cell line combinations which supports future experiments with the choice of the best suitable cell line or serotype. We also provide the information that rAAV5 can only limitedly be tested in vitro but when the gene of interest is an opsin, its functionality can be confirmed in a voltage clamp setup.

In comparison to our reference viruses, we can claim to have a high titer and functional virus production that was proven by ex vivo applications in the next step.

\subsubsection{Application of rAAVs ex vivo and in vivo}

\section{Ex vivo application of rAAV serotypes to elucidate their potential for gene therapy}

Human mesenchymal stem cells - Mesenchymal stem cells (MSCs) have attracted attention as a vehicle for gene therapy in regenerative medicine with their multi-potent ability to differentiate into various cell types, e.g., adipocytes and neurons (Mohammadian et al., 2016).

Analogous to our experiment in which we gained the expression profile of our five rAAV serotypes on immortalized human cell lines, we characterised them on MSCs derived from the Warton's jelly of the umbilical cord of three donors. Interesting to our knowledge was 
the diversity of susceptibility not only between the serotypes but also between the donors. rAAV serotype 2 robustly performed on all three donor MSCs what might explain the preferred usage of this serotype in recent studies (Stender et al., 2007). Nevertheless, the serotype 6 and the chimeric $1 / 2$ partially achieved a higher transduction rate. According to our data presented here, we strongly suggest investigators to elucidate patient-wise which serotype is superior.

As a follow-up to this study we planned to elucidate whether the gene manipulation predifferentiation to neurons and osteoblasts keeps constant or is silenced.

Cortical cultures of the Wistar rat and three non-human primates - With the ethical obligation to minimize the need of in vivo experiments and animals as tissue donors for ex vivo experiments we tested our rAAVs on cortical cultures of the Wistar rat and three non-human primates in the next step for the neuronal delivery. The tissue came from animals which had been euthanised for other reasons and where the cortex was not required for further examinations. This, especially for the non-human primates, limited the excess to the material, especially from young individuals.

Acute cortex slices could be cultivated from all species, whereas dissociated cultures could only be gained from the Wistar rat. As tissue from younger individuals has a more extended viability in culture, we assume that the material from even newborn or adult non-human primates is not suitable to lay out a culture. The expression profile of the five serotypes with constitutive and neuron-specific promotors on dissociated cultures pointed out that the serotype 5 mainly infects glia cells (based on morphological cell type identification). It surprisingly also drives an expression with the CaMKII promotor that is widely claimed as neuron-specific (Taymans et al., 2007). The rAAV2 was the only serotype transducing no cells.

These results are in agreement with Howard et al. (2008) who also found no neuron-related expression using another constitutive, the CMV promotor for rAAV5. They described a late and rare onset of expression for the serotype 2, which might have been out of our observation period.

Expression was only detected in few cases when an opsin was included besides the reporter gene. Our study illustrates that, e.g., serotype 1 and the chimeric 1/2 are broadly effective ex vivo and can also be used in voltage clamp experiments. But we also reasoned that rAAV5 with constructs for neuronal targeting needs to be applied in vivo or, e.g., on cerebral organoid models of the brain (Lancaster et al., 2013). 


\section{In vivo applications}

Transduction of rAAV5 in the mouse cochlea - The cochlea is a well-established target for rAAVs as for the aim to restore hearing with optogenetic implants (György et al., 2017; Moser, 2015). In support of the above-discussed results this offered us the opportunity to assess the in vivo potential of our viral batches. We injected the two high titer viruses that were also addressed in the quality control section (see 4.3) in the cochlea of mice and visualised the transduced cells in immunofluorescence stainings. Both viruses transduced some supporting cells. No high number of transduced cells was expected thus the serotype 5 is none of the preferred serotypes for cochlea transduction. For the CAG promotor, it is published that it can drive a high expression in murine cochlea cells (Liu et al., 2007). Based on the micrographs we did not identify any changes at the injection sites and thereby conclude that the viral samples are safe for application.

Transduction of $\mathrm{rAAV} 5$ in the cortex of Macaca mulatta - In the course of an experiment in our cooperation with the Cognitive Neuroscience Laboratory and the Neurobiology Laboratory (Deutsches Primatenzentrum GmbH, Göttingen), which focused on the injection strategy and viral spread, a purchased and a self-produced virus were injected in the dorsal and ventral pre-motor cortex of a Macaca mulatta. The animal was euthanised ten weeks post-virus injection and the brain regions processed for immunofluorescence stainings. Based on the detected transgene expression of the reference virus, we concluded that the injection procedure was working. For the self-produced virus, no transgene expression related to this virus was visualised in the adjacent neuropil but alterations regarding a lower background fluorescence intensity and a reduction of NeuN positive cells. We can thereby make no conclusions about the virus functionality in the cortex of Macaca mulatta.

The alterations of the neuropil can have various reasons: a mechanic injury caused by the injection, neuropathogen virus infection, ischemia, anoxia or hypoglycemia (Vandevelde et al., 2012). For a detailed investigation of the trigger, advanced histological stainings could be performed to examine, e.g., the glia cells' reaction. Additionally, control injections of the virus sample buffer alone and a control substance, like PBS, could be revealing. 


\section{2 $\mathrm{CD} 9_{\mathrm{GFP}}$ over-expression increases the yield of exo-AAV1}

Since their discovery in 2012 by Maguire and colleagues, exosome-associated AAVs (exoAAVs) have been a promising tool with several advantages over standard rAAVs. They fullfill the requirements for a successful gene-therapy: they have an increased transduction efficiency and an increased resistance against anti-AAV antibodies in vitro and in vivo. Additionally, as for lentiviruses, their lipid membrane can be modified to alter the tropism. The pre-existence of anti-AAV antibodies in the human population and the low transduction efficiency of several targets are major hurdles for gene therapy (Meliani et al., 2017; Maguire et al., 2012).

Many reviews point out the need for improved protocols for a higher yield of rAAVs, which also holds true for exo-AAVs (Meliani et al., 2017). Furthermore, more efficient vectors are required to decrease the high $\mathrm{rAAV}$ doses of up to $10^{14}$ vector genomes per $\mathrm{kg}$ of body weight to achieve a gene expression that is sufficient for the treatment, e.g., in the CNS (György and Maguire, 2017). We examined (as proof of principle) with an exciting outcome how a minor modification in the production protocol can increase the release of exosomes and influence the yield and efficiency of exo-AAVs.

In the publication where I am a contributing author, we found that over-expression of CD9, an exosomal marker-protein, led to an increased release of exosomes in several human cell lines and more efficient lentiviruses (Böker et al., 2017).

Here, we demonstrate that the exogenous expression of $\mathrm{CD} 9_{\mathrm{GFP}}$ in HEK-AAV cells increases the transduction efficiency of exo-AAV1-CD $9_{\mathrm{GFP}}$ for $26 \% \pm 4.9 \%$ (mean $\pm \mathrm{SD}$ ) in comparison with exo-AAV1. We found that the increased transduction efficiency is likely based on the higher yield of vesicle-bound rAAVs.

First of all, we generated HEK-AAV cells by lentiviral transduction that over-express a GFP-fusion protein of the tetraspanin CD9. As previously performed, we isolated the extracellular vesicles using PEG precipitation and additionally with the ultracentrifugation protocol, which is the standard protocol for exo-AAV harvest. We measured the vesicle size and concentration by NTA. Assuming as reported that rAAV production does not influence the vesicle release, we concluded that the same distribution would be found during rAAV1 production, which we could not reassure due to safety precautions (Maguire et al., 2012). However, to mimick rAAV production conditions we transfected the cells with pAAV-CAG-GFP but without the helper plasmid.

As we published for additional cell lines, our NTA data show a decreased size profile of the extracellular vesicles for the $\mathrm{CD} 9_{\mathrm{GFP}}$ over-expressing cell line. And the exosome concentration in the PEG precipitation was increased as well as unproportionally high in the 
$100 \mathrm{x}$ g pellet. The comparison of both methods furthermore revealed that the increased output of exosomes is no artifact of the PEG precipitation.

We then analysed the genomic titers of the exo-AAV harvesting steps (media, $20 \mathrm{k} \mathrm{X} \mathrm{g}$, $100 \mathrm{k} \mathrm{X} \mathrm{g}$, and supernatant) and found no significant differences in vector production between the wild type and $\mathrm{CD} 9_{\mathrm{GFP}}$ over-expressing cells.

Using an iodixanol gradient analysis to distinguish between free and vesicle-bound rAAVs, we found that the percentage of vesicle-bound rAAVs increased upon CD9 $9_{\mathrm{GFP}}$ overexpression. The distribution of free and exosome-bound vectors in the gradient was comparable to other reports for exosomes, rAAVs, and other enveloped viruses, e.g. HIV and Hepatitis C (Böker et al., 2017; György et al., 2014; Liu and Berkhout, 2014; Cantin et al., 2008). The results prompted us to propose that the enhanced transduction efficiency of exo-AAV1-CD9 $9_{\text {GFP }}$ on HEK cells is the consequence of the increased number of vesicle-bound AAVs.

A current study found that another non-enveloped virus, in that case hepatitis, occurs as a membrane associated form comparable to naturally enveloped viruses (which have additional viral glycoproteins in their envelope) and originates from the cellular exosomal pathway (Nagashima et al., 2017). These findings indicate even more that the association of rAAVs and exosomes is not artificially induced by the experimental protocol but found for several viruses.

Similar to the over-expression of $\mathrm{CD} 9_{\mathrm{GFP}}$, the production of exosomes and the release of viruses could be increased by the expression of the viral glycoprotein VSV-G or treatment of hepatitis E producing cells with the lysosomal inhibitor bafilomycin A1. In contrast, a knock-down of the exosome machinery reduced the release of viruses to the media (Maguire et al., 2012; Nagashima et al., 2014; Alvarez-Erviti et al., 2011). These results already demonstrate that positive modulation of the cellular exosome pathway can be used to alter the amount of released, vesicle-associated viruses. For the first time we demonstrated that this also happens for exo-AAV1 and we hypothesize that a combined approach with additional administration of bafilomycin A1 might further increase the exosome and exo-AAV1 production.

Future experiments should clarify the biophysical properties of the high-CD9 vesicles and disclose whether the tetraspanin CD9 stabilizes the lipid membrane, enhances the interaction with the rAAVs or mediates a more efficient fusion with the target cell. We therefore provide a rAAV production and test pipeline, as well as rAAV and lentivirus plasmids with the same genetic information (see 3.1 and 3.3) which can serve to unravel the mode of action that is currently unknown. 
As we consider our experiments as a proof-of-principle, we used our established production protocol for standard rAAVs. This uses the helper-free system of E1 transformed HEK293 cells for virus production. Due to the potential loss of the transgene after many passages, it requires work with low-passage cells. And thereby it prevented the complete selection of $\mathrm{CD} 9_{\mathrm{GFP}}-$ positive cells.

Therefore, we aim to generate a stable HEK cell line with concurrent, homogenous expression of $\mathrm{CD} 9_{\mathrm{GFP}}$ and $\mathrm{E} 1$ with the purpose to further increase the exo-AAV yield and minimize the required experimental handling.

\subsection{1 exo-AAV serotypes 1 and 6 reveal a different expression profile on mesenchymal stem cells than standard rAAVs}

We then evaluated how the exo-AAVs of serotype 1 and 6 performed on MSCs and thereby compared vectors produced in normal exosome-level and boosted exosome-level cells.

For the two donors and two serotypes we observed no clear difference whether the exoAAV or exo-AAV-CD9 $9_{\text {GFP }}$ are more efficient. Whereas standard rAAV serotypes 1 and 6 performed similarly robust on donor \#97 (see 3.1.3), both, exo-AAV1 and exo-AAV1$\mathrm{CD} 9_{\mathrm{GFP}}$ performed drastically worse.

Recently, Meliani et al. (2017) hypothesized after competition experiments that exo-AAVs enter the cells through a process that is independent of the serotype-specific AAV-binding receptors. Hence, exo-AAV serotypes 1 and 6 should perform similarly. They additionally showed that the nuclear translocation rate influences the expression level of rAAVs (in their case exo-AAVs had been trafficked more efficiently than the standard rAAVs). Therefore we might hypothesize that upon covering of the native tropism by encapsulation in exosomes, rAAV6 has a more efficient trafficking to the nucleus and therefore a higher transduction efficiency on the MSCs.

If the transduction is mediated by the exosomal envelope, we can conclude that the producer cell line also influences the efficiency or the tropism of the exo-AAVs. Therefore, the choice of another producer cell line might further increase the efficiency of exo-AAVs on MSCs. 


\subsection{Lentiviruses as neuronal delivery tool}

Lentiviruses are the tool of choice when a high transduction rate with a fast and stable protein expression is desired. This makes them a useful tool when new gene constructs, e.g., for optogenetics, should be tested ex vivo and the desired vector, like a rAAV, is not suitable (see 3.1.3). Our generated optogenetic toolbox (see 3.1.2) makes this even easier. In addition to the test of new genes, new retargeting molecules for VLPs or exo-AAVs can be tested with lentiviruses when expressed in the membrane of the producer cell line. Furthermore, the generated lentiviruses are useful to investigate together with the exoAAV-CD $9_{\mathrm{GFP}}$ the mode of action of CD9, with which it increases the infectivity of lentiviruses and the yield of exo-AAVs (see Böker et al. (2017) and 3.2). 


\subsection{Virus-like particles as delivery tool for neuronal targets}

JC polyoma virus-derived VP1 VLPs (in short VLPs) are an exciting tool for research and potentially application in clinics, which require a save and efficient delivery of the therapeutic compound. Its advantages over viral delivery tools, like rAAVs and lentiviruses, are that the recombinant VP1 protein is purified to homogeneity and loaded in an in vitro disassembly/reassembly procedure (Goldmann et al., 1999). Thereby, the uncontrolled loading of cellular proteins, toxins or genetic material from the expression system is avoided. The DNA- or RNA-cargos do not require viral sequences thus the encapsulation is sequence independent (Moreland et al., 1991; Ou et al., 1999). Additionally, VLPs induced no immune response in in vivo studies (Goldmann et al. (1999) and unpublished data) and achieved a successful siRNA delivery for gene knock-down in an osteoporosis rat model (Hoffmann et al., 2016). With these findings, we considered its usability to deliver genetic material into nervous tissue. This included utilisation of its native tropism as well as aiming for a neuronal retargeting.

\subsubsection{Safe DNA delivery by combination of VLPs and MIDGE vectors}

As VLPs were mainly used for artificial RNA transfer in our group, we firstly constructed a DNA expression cassette for easy visualization of transgene expression. Analogous to the pAAVs and pLentiviruses (see 3.1 and 3.3) this plasmid uses a CAG-promotor to drive GFP expression, and contains the WPRE sequence. Previous studies determined approximately $3.5 \mathrm{~kb}$ as the payload limit for the VLPs. Because most plasmids exceed this size (see appendix 5.1), two restriction sites within the plasmid enable an enzymatic digestion to gain a linear expression cassette of $2.6 \mathrm{~kb}$ in length.

We also aimed to eliminate the $\mathrm{CpG}$ motif-rich bacterial plasmid backbone because it bears the risk of immunological side effects, as well as the open ends of linear DNA do (Krieg, 1999). To adress the second threat, we generated so-called MIDGE vectors to generate a monomolecular, linear DNA (Schakowski et al., 2001).

Our results showed comparable transduction rates of SKBR3 cells for the linear and the re-circularized construct (see 3.4.1).

Since we identified the production of these MIDGE vectors to be very time- and costintensive, we claim this approach as proof-of-principle. We intend to utilise MIDGE vectors in upcoming in vivo studies with VLPs. Additionally, the use of the dual- or the recently published triple AAV-strategy might bear a potential to transfer expression cassettes for bigger proteins (McClements and MacLaren, 2017; Maddalena et al., 2017). Such an approach would eliminate the size limitations of VLPs and broaden their usage 
for gene therapy approaches where larger transgenes are required.

\subsubsection{Neuronal expression and retargeting of VLPs}

For the neuronal delivery and transgene expression we followed two approaches:

- the restriction of expression by a neuronal promotor, enabling a broad delivery

- retargeting of VLPs, which would allow the targeted delivery of a strong expression promotor

Similar to the rAAVs and lentiviruses which we tested for the activity of the CaMKII and the hSyn promotors in HeLa cells (see 3.1), we used linear DNA derived from the pAAVs and delivered them via VLPs. We previously observed that VLPs well transduce HeLa cells and lead to a GFP-expression driven by the CAG promotor (see 3.4.2). But none of both constructs with the neuron specific promotors led to a transgene expression. We conclude that the lack of expression was due to the combination of the rather weak promotors and the delivery via VLPs.

To exclude cell line artifacts, we tested the VLPs, similar to the rAAVs (see 3.1.3), on primary cortical cultures of the Wistar rat. No GFP-expression was detectable and the culture viability decreased dramatically in a dose-dependent manner. Pre-equilibration and re-buffering of the VLP sample pre-application could not circumvent this effect.

Thus we could transduce primary rat osteoblasts and the neuroblastoma cell line SHSY5Y with VLPs and detect expression of the CAG-GFP construct, it could be explained by the high sensitivity of neuronal cultures. Supported by the previous observation that a culture of Callithrix jacchus glia cells was susceptible for VLPs delivering a flourescence dye (Cy3) coupled small RNA.

We conclude that the ability of neuronal targeting with VLPs has to be elucidated in another ex vivo model which is more robust. Possibly a denser cell structure, like in cerebral organoid models of the human brain, supports the cell viability after the transduction with VLPs (Lancaster et al., 2013). VLPs were found to work efficiently in vivo without harming the individuum (Hoffmann et al., 2016). An in vivo study using MIDGE vector-loaded VLPs with both, constitutive and neuron-specific promotors and subsequent analysis of organs and cell types for the reporter gene expression would help to unravel the transduction profile of VLPs.

Another option to achieve a cell type restricted protein expression is, as previously described, the use of promotors being inactive in off-target tissues (White et al., 2009). The 
option which we provide for the targeting of Her2/neu-receptor positive cell lines (cancer cell lines) is the modification of the VLPs ${ }^{6}$ tropism via the attachment of retargeting molecules. Having developed a functional retargeting molecule, this can also be used to alter the tropism of exo-AAVs and lentiviruses. For these two membrane-enveloped viruses, the retargeting molecule would be recombinantly expressed as a membrane-anchored protein in the virus producer cell line. When the virus is released from the cell, it will gain the membraneuos envelope with the exposed retargeting molecule. Taken together, one could compare the three tools and identify the best suitable one for a specific application. The humanized strain of the yeast $P$. pastoris stands out due to its humanized glycosylation profile of recombinant expressed proteins in a sense that only a 5 times branching of mannose is seen (Hamilton and Gerngross, 2007). Thereby it is our preferred expression system for retargeting molecule expression, which we used to express the Her2/neu single chain variable fragments ( $\mathrm{scFv}$ ) that altered the VLPs' tropism towards human cancer cells (see 3.4.2). We constructed the expression plasmid for a scFv that should bind to the tropomyosin receptor kinase B (TrkB), the receptor for BDNF which is expressed in the central and peripheral nervous system and other tissues (Gupta et al., 2013). Unfortunately, no recombinant protein expressing P. pastoris clone could be identified in repeated attempts.

Hence, we switched the expression constructs to the pET28- E.coli expression system and achieved the expression of the retargeting molecules. However, the retargeting molecules were unsoluble. The protein transcription rate and the folding of proteins expressed in E. coli can be influenced by several cultivation parameters. In our case, we gained unsoluble proteins even when reducing the transcription rate by lowering the concentration of the induction reagent IPTG or by decreasing the temperature and thereby increasing the possibility of proper folding of a soluble protein (Schein and Noteborn, 1988).

Alternative approaches to gain a soluble protein would be the use of bacteria strains which secrete the protein to the periplasm or the medium, or the co-expression of chaperons to gain an improved protein folding (Mergulhao et al., 2005; De Marco, 2009). Additionally, one could try to express the protein in insect cells as we do for the VP1 protein or in human cell lines. These alternative approaches were not undertaken due to the restricted time in the course of this study.

As a promising alternative, we tested a synthetic peptide derived from the rabies virus glycoprotein (RVG) that was shown to specifically deliver siRNAs through the blood-brain barrier (Javed et al., 2016). RVG binds to the n-acetylcholine receptor which is expressed, e.g., in the neuroblastoma cell line SH-SY5Y but not in fibroblasts (Lentz, 1990; Javed et al., 2016; Kovalevich and Langford, 2013). In our experiments, the native VLP trans- 
duced both cell lines and SKBR3 cells were used as our positive control for functional VLPs. VLPs with the attached crosslinker alone, as well as with the crosslinker and the retargeting molecule showed drastically reduced transduction of all cell lines. Based on this result we can make no conclusion whether the crosslinking of the retargeting molecule did not work or the molecule was crosslinked but did not bind to the cells.

Further experiments with an altered crosslinking protocol to enhance the crosslinkingsuccess will be elucidated. Because we utilised a rather short crosslinker, steric hindrance might have not allowed the small RVG-proteins (scFvs are several folds larger) to bind to the cell receptors when attached on the VLPs' surface. The use of a longer crosslinker than the used one of 32.5 angstroms, might be successful.

In summary, we demonstrated that VLPs are a very useful, non-viral tool to deliver DNA expression cassettes in vitro. We combined this technology with MIDGE vectors, which will increase the safety of DNA delivery in in vivo applications. Even if we could not achieve neuronal retargeted VLPs, our results contribute to the previous finding of specific delivery of therapeutic expression cassettes via Her2/neuscFv-retargeted VLPs into cancers cells. 


\subsection{Conclusion and future directives}

Gene therapy is a rising field in which the engineering of the delivery tools holds a big promise to improve its efficiency and safety.

In this context, we worked on three delivery tools: the viral rAAVs and lentiviruses which are well-established in gene-therapy, and the non-viral JC polyoma virus-derived VLPs which are a promising tool. Our focus was on the rather new technology of exo-AAVs which outperform standard AAVs regarding the transduction efficiency and the antibody evasion.

Within this study, we provide a platform for a straightforward generation of expression constructs with our genetic toolbox and the initiation of retargeting strategies which are applicable for most of the tools. As the target, we chose nervous tissue with the exemplary application of optogenetics, as we work in a cooperation that addresses neuro-optogenetics in the non-human primate.

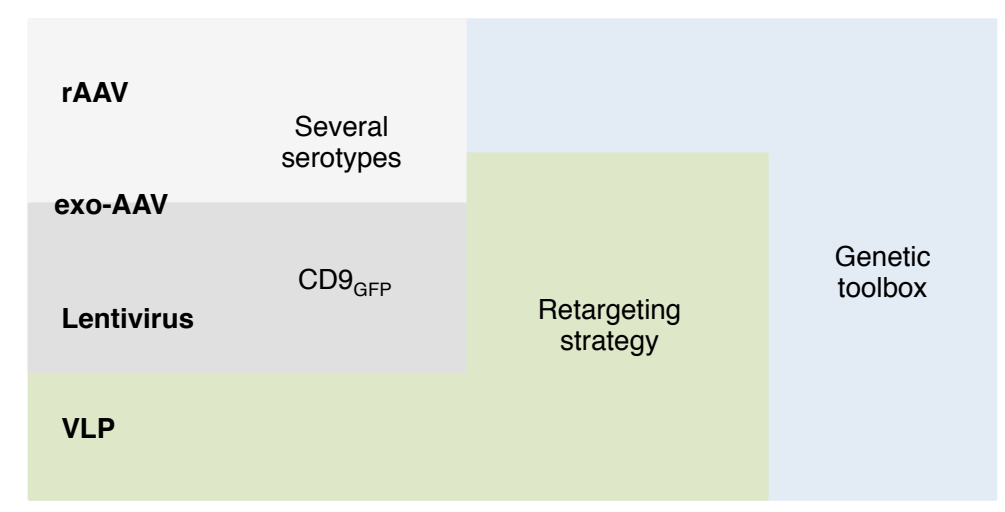

Figure 33: Crosslink of engineering strategies Details described in the text.

Firstly we set up a pipeline for in vivo grade standard rAAV5 purification with in vitro quality control and ex vivo tests which can be applied to further serotypes and exo-AAVs as well. Each rAAV serotype exhibits a different tropism and expression pattern. We provide expression data for several serotypes with a constitutive and neuronal promotors on human cells lines as well as on primary cells and tissue of human, rodent and non-human primate origin.

One challenge for commercial gene therapy is the production of the delivery tools in a high yield. For the first time we show that exo-AAVs produced in an exosome-enriched environment have an approximately $26 \%$ increased efficiency. To achieve this, we applied our previous finding that the over-expression of the exosomal marker-protein CD9 increases the exosome production in human cell lines. Moreover, our data indicate that 
the higher efficiency is due to an increased number of exosome bound viruses while the overall secretion of rAAVs in the media stayed constant. By that, we provide a simple and economical protocol to produce around $25 \%$ more exo-AAVs with a minor additional effort.

Furthermore, we worked on a biochemical retargeting approach of VLPs towards neurons by the attachment of retargeting molecules for two neuronal receptors. This approach could be applied for exo-AAVs and lentiviruses as well. However, the successful retargeting has to be proven in the future. By combining the VLP-technology and MIDGE vectors, we point out a safe way to deliver linear expression constructs which are less potent to cause an immune response.

Together, these developments provide a handy way to improve delivery vectors which can be tested and compared in the set up pipeline which will allow choosing the most efficient one for future applications. 


\section{Bibliography}

Catherine S Adamson and Ian M Jones. The molecular basis of hiv capsid assembly - five years of progress. Reviews in medical virology, 14(2):107-121, 2004.

Alessandro Aiuti, Luca Biasco, Samantha Scaramuzza, Francesca Ferrua, Maria Pia Cicalese, Cristina Baricordi, Francesca Dionisio, Andrea Calabria, Stefania Giannelli, Maria Carmina Castiello, et al. Lentiviral hematopoietic stem cell gene therapy in patients with wiskott-aldrich syndrome. Science, 341(6148):1233151, 2013.

Alessandro Aiuti, Maria Grazia Roncarolo, and Luigi Naldini. Gene therapy for ada-scid, the first marketing approval of an ex vivo gene therapy in europe: paving the road for the next generation of advanced therapy medicinal products. EMBO Molecular Medicine, page e201707573, 2017.

M Lucrecia Alvarez, Mahdieh Khosroheidari, Rupesh Kanchi Ravi, and Johanna K DiStefano. Comparison of protein, microrna, and mrna yields using different methods of urinary exosome isolation for the discovery of kidney disease biomarkers. Kidney international, 82(9):1024-1032, 2012.

Lydia Alvarez-Erviti, Yiqi Seow, Anthony H Schapira, Chris Gardiner, Ian L Sargent, Matthew JA Wood, and J Mark Cooper. Lysosomal dysfunction increases exosome-mediated alpha-synuclein release and transmission. Neurobiology of disease, 42(3):360-367, 2011.

T.J. Antes, K. Kwei, and F. Wu. Methods for microvesicle isolation and selective removal, April 142015. URL https://www.google.com/patents/US9005888. US Patent 9,005,888.

Erik Arden and Joseph M Metzger. Inexpensive, serotype-independent protocol for native and bioengineered recombinant adeno-associated virus purification. Journal of biological methods, 3(2), 2016.

Aristides B Arrenberg, Didier YR Stainier, Herwig Baier, and Jan Huisken. Optogenetic control of cardiac function. Science, 330(6006):971-974, 2010.

Aarthi Ashok and Walter J Atwood. Contrasting roles of endosomal ph and the cytoskeleton in infection of human glial cells by jc virus and simian virus 40. Journal of virology, 77(2):1347-1356, 2003.

Robert W Atchison, Bruce C Casto, and William McD Hammon. Adenovirus-associated defective virus particles. Science, 149(3685):754-755, 1965.

MB Banasik and PB McCray. Integrase-defective lentiviral vectors: progress and applications. Gene therapy, 17(2):150-157, 2010.

Jeffrey S Bartlett, Rose Wilcher, and R Jude Samulski. Infectious entry pathway of adeno-associated virus and adeno-associated virus vectors. Journal of virology, 74(6):2777-2785, 2000. 
Antonette Bennett, Saajan Patel, Mario Mietzsch, Ariana Jose, Bridget Lins-Austin, C Yu Jennifer, Brian Bothner, Robert McKenna, and Mavis Agbandje-McKenna. Thermal stability as a determinant of aav serotype identity. Molecular Therapy-Methods \& Clinical Development, 6:171-182, 2017.

KI Berns and JA Rose. Evidence for a single-stranded adenovirus-associated virus genome: isolation and separation of complementary single strands. Journal of virology, 5(6):693-699, 1970.

Sangeeta N Bhatia and Donald E Ingber. Microfluidic organs-on-chips. Nature biotechnology, 32(8): 760-772, 2014.

Alessandra Biffi, Eugenio Montini, Laura Lorioli, Martina Cesani, Francesca Fumagalli, Tiziana Plati, Cristina Baldoli, Sabata Martino, Andrea Calabria, Sabrina Canale, et al. Lentiviral hematopoietic stem cell gene therapy benefits metachromatic leukodystrophy. Science, 341(6148):1233158, 2013.

Cherie Blenkiron, Denis Simonov, Anita Muthukaruppan, Peter Tsai, Priscila Dauros, Sasha Green, Jiwon Hong, Simon Swift, Anthony R Phillips, et al. Uropathogenic escherichia coli releases extracellular vesicles that are associated with rna. PloS one, 11(8):e0160440, 2016.

Kai O Böker, Nicolas Lemus-Diaz, Rafael Rinaldi Ferreira, Lara Schiller, Stefan Schneider, and Jens Gruber. The impact of the cd9 tetraspanin on lentivirus infectivity and exosome secretion. Molecular Therapy, 2017.

K Bourzac. In first human test of optogenetics, doctors aim to restore sight to the blind. MIT Technology Review, 2016.

Otmane Boussif, Frank Lezoualc'h, Marla Aantonietta Zanta, Mojgan Djavaheri Mergny, Daniel Scherman, Barbara Demeneix, and Jean-Paul Behr. A versatile vector for gene and oligonucleotide transfer into cells in culture and in vivo: polyethylenimine. Proceedings of the National Academy of Sciences, 92(16):7297-7301, 1995.

Edward S Boyden, Feng Zhang, Ernst Bamberg, Georg Nagel, and Karl Deisseroth. Millisecond-timescale, genetically targeted optical control of neural activity. Nature neuroscience, 8(9):1263-1268, 2005.

Dirk G Brockstedt, Greg M Podsakoff, Lawrence Fong, Gary Kurtzman, Wolfgang Mueller-Ruchholtz, and Edgar G Engleman. Induction of immunity to antigens expressed by recombinant adeno-associated virus depends on the route of administration. Clinical Immunology, 92(1):67-75, 1999.

Gary L Buchschacher and Flossie Wong-Staal. Development of lentiviral vectors for gene therapy for human diseases. Blood, 95(8):2499-2504, 2000.

Michael I Bukrinsky, Sheryl Haggerty, Michael P Dempsey, Natalia Sharova, Alexei Adzhubei, Lynn Spitz, Paul Lewis, David Goldfarb, Michael Emerman, and Mario Stevenson. A nuclear localization signal within hiv-1 matrix protein that governs infection of non-dividing cells. Nature, 365(6447): 666-669, 1993.

Jane C Burns, Theodore Friedmann, Wolfgang Driever, Michelle Burrascano, and Jiing-Kuan Yee. Vesicular stomatitis virus g glycoprotein pseudotyped retroviral vectors: concentration to very high titer and efficient gene transfer into mammalian and nonmammalian cells. Proceedings of the National Academy of Sciences, 90(17):8033-8037, 1993. 
Roberto Calcedo, Judith Franco, Qiuyue Qin, Dean W Richardson, Jeffery B Mason, Surina Boyd, and James M Wilson. Preexisting neutralizing antibodies to adeno-associated virus capsids in large animals other than monkeys may confound in vivo gene therapy studies. Human gene therapy methods, 26(3): 103-105, 2015.

RSF Campbell and WF Robinson. The comparative pathology of the lentiviruses. Journal of comparative pathology, 119(4):333-395, 1998.

Réjean Cantin, Juliette Diou, Dave Bélanger, Alexandre M Tremblay, and Caroline Gilbert. Discrimination between exosomes and hiv-1: purification of both vesicles from cell-free supernatants. Journal of immunological methods, 338(1):21-30, 2008.

Barrie J Carter. Adeno-associated virus and the development of adeno-associated virus vectors: a historical perspective. Molecular therapy, 10(6):981-989, 2004.

Nathalie Cartier, Salima Hacein-Bey-Abina, Cynthia C Bartholomae, Gabor Veres, Manfred Schmidt, Ina Kutschera, Michel Vidaud, Ulrich Abel, Liliane Dal-Cortivo, Laure Caccavelli, et al. Hematopoietic stem cell gene therapy with a lentiviral vector in x-linked adrenoleukodystrophy. science, 326(5954): 818-823, 2009.

Antoine Chaffiol, Romain Caplette, Céline Jaillard, Elena Brazhnikova, Mélissa Desrosiers, Elisabeth Dubus, Laëtitia Duhamel, Emilie Macé, Olivier Marre, Patrick Benoit, et al. A new promoter allows optogenetic vision restoration with enhanced sensitivity in macaque retina. Molecular Therapy, 2017.

Chi-Fang Chang, Meilin Wang, Wei-Chih Ou, Pei-Lain Chen, Cheng-Huang Shen, Paul Yann Lin, ChiungYao Fang, and Deching Chang. Human jc virus-like particles as a gene delivery vector. Expert opinion on biological therapy, 11(9):1169-1175, 2011.

Deching Chang, Chiung-Yau Fung, Wei-Chih Ou, Pei-Chi Chao, Shuan-Yow Li, Meilin Wang, Yih-Leh Huang, Tsair-Yuh Tzeng, and Rong-Tai Tsai. Self-assembly of the jc virus major capsid protein, vp1, expressed in insect cells. Journal of general virology, 78(6):1435-1439, 1997.

John C Chapin and Paul Edward Monahan. Gene therapy for hemophilia: Progress to date. BioDrugs, pages $1-17,2017$.

Pei-Lain Chen, Meilin Wang, Wei-Chih Ou, Chong-Kuei Lii, Li-Sheng Chen, and Deching Chang. Disulfide bonds stabilize jc virus capsid-like structure by protecting calcium ions from chelation. FEBS letters, 500(3):109-113, 2001.

Lesley Cheng, Xin Sun, Benjamin J Scicluna, Bradley M Coleman, and Andrew F Hill. Characterization and deep sequencing analysis of exosomal and non-exosomal mirna in human urine. Kidney international, 86(2):433-444, 2014.

Mitsuru Chiba, Misako Kimura, and Saya Asari. Exosomes secreted from human colorectal cancer cell lines contain mrnas, micrornas and natural antisense rnas, that can transfer into the human hepatoma hepg2 and lung cancer a549 cell lines. Oncology reports, 28(5):1551-1558, 2012. 
Hansang Cho, Ji Hae Seo, Keith HK Wong, Yasukazu Terasaki, Joseph Park, Kiwan Bong, Ken Arai, Eng H Lo, and Daniel Irimia. Three-dimensional blood-brain barrier model for in vitro studies of neurovascular pathology. Scientific reports, 5, 2015.

Nathalie Clément and Joshua C Grieger. Manufacturing of recombinant adeno-associated viral vectors for clinical trials. Molecular Therapy-Methods \& Clinical Development, 3:16002, 2016.

Jason E Coleman, Matthew J Huentelman, Sergey Kasparov, Beverly L Metcalfe, Julian FR Paton, Michael J Katovich, Susan L Semple-Rowland, and Mohan K Raizada. Efficient large-scale production and concentration of hiv-1-based lentiviral vectors for use in vivo. Physiological Genomics, 12(3): $221-228,2003$.

Ronald G Crystal. Adenovirus: the first effective in vivo gene delivery vector. Human gene therapy, 25 (1):3-11, 2014.

Deniz Dalkara, Kathleen D Kolstad, Natalia Caporale, Meike Visel, Ryan R Klimczak, David V Schaffer, and John G Flannery. Inner limiting membrane barriers to aav-mediated retinal transduction from the vitreous. Molecular Therapy, 17(12):2096-2102, 2009.

Ario De Marco. Strategies for successful recombinant expression of disulfide bond-dependent proteins in escherichia coli. Microbial cell factories, 8(1):26, 2009.

Christophe Delenda. Lentiviral vectors: optimization of packaging, transduction and gene expression. The journal of gene medicine, 6(S1), 2004.

Markus Dettenhofer and Xiao-Fang Yu. Highly purified human immunodeficiency virus type 1 reveals a virtual absence of vif in virions. Journal of virology, 73(2):1460-1467, 1999.

Ilka Diester, Matthew T Kaufman, Murtaza Mogri, Ramin Pashaie, Werapong Goo, Ofer Yizhar, Charu Ramakrishnan, Karl Deisseroth, and Krishna V Shenoy. An optogenetic toolbox designed for primates. Nature neuroscience, 14(3):387-397, 2011.

Rebecca A Dragovic, Christopher Gardiner, Alexandra S Brooks, Dionne S Tannetta, David JP Ferguson, Patrick Hole, Bob Carr, Christopher WG Redman, Adrian L Harris, Peter J Dobson, et al. Sizing and phenotyping of cellular vesicles using nanoparticle tracking analysis. Nanomedicine: Nanotechnology, Biology and Medicine, 7(6):780-788, 2011.

Sandra Duque, Béatrice Joussemet, Christel Riviere, Thibaut Marais, Laurence Dubreil, Anne-Marie Douar, John Fyfe, Philippe Moullier, Marie-Anne Colle, and Martine Barkats. Intravenous administration of self-complementary aav9 enables transgene delivery to adult motor neurons. Molecular Therapy, 17(7):1187-1196, 2009.

S Eash, K Manley, M Gasparovic, W Querbes, and WJ Atwood. The human polyomaviruses. Cellular and Molecular Life Sciences CMLS, 63(7-8):865-876, 2006.

Gwendolyn F Elphick, William Querbes, Joslynn A Jordan, Gretchen V Gee, Sylvia Eash, Kate Manley, Aisling Dugan, Megan Stanifer, Anushree Bhatnagar, Wesley K Kroeze, et al. The human polyomavirus, jcv, uses serotonin receptors to infect cells. Science, 306(5700):1380-1383, 2004. 
Michael W Ferenczy, Leslie J Marshall, Christian DS Nelson, Walter J Atwood, Avindra Nath, Kamel Khalili, and Eugene O Major. Molecular biology, epidemiology, and pathogenesis of progressive multifocal leukoencephalopathy, the jc virus-induced demyelinating disease of the human brain. Clinical microbiology reviews, 25(3):471-506, 2012.

Forrest K Ferrari, Thaddeus Samulski, Thomas Shenk, and Richard Jude Samulski. Second-strand synthesis is a rate-limiting step for efficient transduction by recombinant adeno-associated virus vectors. Journal of virology, 70(5):3227-3234, 1996.

Gerald D Fischbach. Synapse formation between dissociated nerve and muscle cells in low density cell cultures. Developmental biology, 28(2):407-429, 1972.

Krishna J Fisher, Guang-Ping Gao, Matthew D Weitzman, Ronald DeMatteo, John F Burda, and James M Wilson. Transduction with recombinant adeno-associated virus for gene therapy is limited by leading-strand synthesis. Journal of virology, 70(1):520-532, 1996.

Zachary Fitzpatrick, Bence György, Johan Skog, and Casey A Maguire. Extracellular vesicles as enhancers of virus vector-mediated gene delivery. Human gene therapy, 25(9):785-786, 2014.

Kevin D Foust, Emily Nurre, Chrystal L Montgomery, Anna Hernandez, Curtis M Chan, and Brian K Kaspar. Intravascular aav9 preferentially targets neonatal neurons and adult astrocytes. Nature biotechnology, 27(1):59-65, 2009.

Nicholas R Franich, Helen L Fitzsimons, Dahna M Fong, Matthias Klugmann, Matthew J During, and Deborah Young. Aav vector-mediated rnai of mutant huntingtin expression is neuroprotective in a novel genetic rat model of huntington's disease. Molecular Therapy, 16(5):947-956, 2008.

Thorsten Friedel, Lydia J Hanisch, Anke Muth, Annemarie Honegger, Hinrich Abken, Andreas Plückthun, Christian J Buchholz, and Irene C Schneider. Receptor-targeted lentiviral vectors are exceptionally sensitive toward the biophysical properties of the displayed single-chain fv. Protein Engineering, Design and Selection, 28(4):93-106, 2015.

Richard J Frisque, Gary L Bream, and Maria T Cannella. Human polyomavirus jc virus genome. Journal of virology, 51(2):458-469, 1984.

P Garberg, Marguerite Ball, N Borg, Roméo Cecchelli, Laurence Fenart, RD Hurst, T Lindmark, Aloïse Mabondzo, JE Nilsson, TJ Raub, et al. In vitro models for the blood-brain barrier. Toxicology in vitro, 19(3):299-334, 2005.

Juan José Garrido, Maria Teresa Alonso, Filip Lim, Estela Carnicero, Fernando Giraldez, and Thomas Schimmang. Defining responsiveness of avian cochlear neurons to brain-derived neurotrophic factor and nerve growth factor by hsv-1-mediated gene transfer. Journal of neurochemistry, 70(6):2336-2346, 1998.

Anja Geisler and Henry Fechner. Microrna-regulated viral vectors for gene therapy. World journal of experimental medicine, 6(2):37, 2016. 
Annelies Gerits, Reza Farivar, Bruce R Rosen, Lawrence L Wald, Edward S Boyden, and Wim Vanduffel. Optogenetically induced behavioral and functional network changes in primates. Current Biology, 22 (18):1722-1726, 2012.

Annelies Gerits, Pascaline Vancraeyenest, Samme Vreysen, Marie-Eve Laramée, Annelies Michiels, Rik Gijsbers, Chris Van den Haute, Lieve Moons, Zeger Debyser, Veerle Baekelandt, et al. Serotypedependent transduction efficiencies of recombinant adeno-associated viral vectors in monkey neocortex. Neurophotonics, 2(3):031209-031209, 2015.

Hubert W Gerry, Thomas J Kelly, and Kenneth I Berns. Arrangement of nucleotide sequences in adenoassociated virus dna. Journal of molecular biology, 79(2):207IN1213-212225, 1973.

ET Gillock, S Rottinghaus, D Chang, X Cai, SA Smiley, K An, and RA Consigli. Polyomavirus major capsid protein vp1 is capable of packaging cellular dna when expressed in the baculovirus system. Journal of virology, 71(4):2857-2865, 1997.

Stefan Gleiter and Hauke Lilie. Coupling of antibodies via protein z on modified polyoma virus-like particles. Protein Science, 10(2):434-444, 2001.

Claudia Goldmann, Harald Petry, Stephan Frye, Oliver Ast, Susanne Ebitsch, Klaus-Dieter Jentsch, Franz-Josef Kaup, Frank Weber, Corinna Trebst, Thomas Nisslein, et al. Molecular cloning and expression of major structural protein vp1 of the human polyomavirus jc virus: formation of virus-like particles useful for immunological and therapeutic studies. Journal of virology, 73(5):4465-4469, 1999.

Steven J Gray, Stacey B Foti, Joel W Schwartz, Lavanya Bachaboina, Bonnie Taylor-Blake, Jennifer Coleman, Michael D Ehlers, Mark J Zylka, Thomas J McCown, and R Jude Samulski. Optimizing promoters for recombinant adeno-associated virus-mediated gene expression in the peripheral and central nervous system using self-complementary vectors. Human gene therapy, 22(9):1143-1153, 2011.

B Greenberg, J Butler, GM Felker, P Ponikowski, AA Voors, JM Pogoda, R Provost, J Guerrero, RJ Hajjar, and KM Zsebo. Prevalence of aav1 neutralizing antibodies and consequences for a clinical trial of gene transfer for advanced heart failure. Gene therapy, 23(3):313-319, 2016.

Joshua C Grieger, Stephen M Soltys, and Richard Jude Samulski. Production of recombinant adenoassociated virus vectors using suspension hek293 cells and continuous harvest of vector from the culture media for gmp fix and ftt1 clinical vector. Molecular Therapy, 24(2):287-297, 2016.

Andreas Gruber, June Kan-Mitchell, Kelli L Kuhen, Tetsu Mukai, and Flossie Wong-Staal. Dendritic cells transduced by multiply deleted hiv-1 vectors exhibit normal phenotypes and functions and elicit an hiv-specific cytotoxic t-lymphocyte response in vitro. Blood, 96(4):1327-1333, 2000.

Lisa A Gunaydin, Ofer Yizhar, André Berndt, Vikaas S Sohal, Karl Deisseroth, and Peter Hegemann. Ultrafast optogenetic control. Nature neuroscience, 13(3):387-392, 2010.

Vivek K Gupta, Yuyi You, Veer Bala Gupta, Alexander Klistorner, and Stuart L Graham. Trkb receptor signalling: implications in neurodegenerative, psychiatric and proliferative disorders. International journal of molecular sciences, 14(5):10122-10142, 2013. 
Bence György and Casey A Maguire. Extracellular vesicles: nature's nanoparticles for improving gene transfer with adeno-associated virus vectors. Wiley Interdisciplinary Reviews: Nanomedicine and Nanobiotechnology, 2017.

Bence György, Zachary Fitzpatrick, Matheus HW Crommentuijn, Dakai Mu, and Casey A Maguire. Naturally enveloped aav vectors for shielding neutralizing antibodies and robust gene delivery in vivo. Biomaterials, 35(26):7598-7609, 2014.

Bence György, Cyrille Sage, Artur A Indzhykulian, Deborah I Scheffer, Alain R Brisson, Sisareuth Tan, Xudong Wu, Adrienn Volak, Dakai Mu, Panos I Tamvakologos, et al. Rescue of hearing by gene delivery to inner-ear hair cells using exosome-associated aav. Molecular Therapy, 25(2):379-391, 2017.

Stephen R Hamilton and Tillman U Gerngross. Glycosylation engineering in yeast: the advent of fully humanized yeast. Current opinion in biotechnology, 18(5):387-392, 2007.

Xue Han. Optogenetics in the nonhuman primate. Progress in brain research, 196:215, 2012.

Xue Han and Edward S Boyden. Multiple-color optical activation, silencing, and desynchronization of neural activity, with single-spike temporal resolution. PloS one, 2(3):e299, 2007.

Xue Han, Brian Y Chow, Huihui Zhou, Nathan C Klapoetke, Amy Chuong, Reza Rajimehr, Aimei Yang, Michael V Baratta, Jonathan Winkle, Robert Desimone, et al. A high-light sensitivity optical neural silencer: development and application to optogenetic control of non-human primate cortex. Frontiers in systems neuroscience, 5, 2011.

Hans C Helms, N Joan Abbott, Malgorzata Burek, Romeo Cecchelli, Pierre-Olivier Couraud, Maria A Deli, Carola Förster, Hans J Galla, Ignacio A Romero, Eric V Shusta, et al. In vitro models of the blood-brain barrier: an overview of commonly used brain endothelial cell culture models and guidelines for their use. Journal of Cerebral Blood Flow \& Metabolism, 36(5):862-890, 2016.

Martin E Hemler. Tetraspanin functions and associated microdomains. Nature reviews Molecular cell biology, 6(10):801-811, 2005.

Wim TJMC Hermens, Olivier Ter Brake, Paul A Dijkhuizen, Marc AF Sonnemans, Dirk Grimm, Jurgen A Kleinschmidt, and Joost Verhaagen. Purification of recombinant adeno-associated virus by iodixanol gradient ultracentrifugation allows rapid and reproducible preparation of vector stocks for gene transfer in the nervous system. Human gene therapy, 10(11):1885-1891, 1999.

Jochen Heukeshoven and Rudolf Dernick. Simplified method for silver staining of proteins in polyacrylamide gels and the mechanism of silver staining. Electrophoresis, 6(3):103-112, 1985.

Niwa Hitoshi, Yamamura Ken-ichi, and Miyazaki Jun-ichi. Efficient selection for high-expression transfectants with a novel eukaryotic vector. Gene, 108(2):193-199, 1991.

Michael Hocquemiller, Laura Giersch, Mickael Audrain, Samantha Parker, and Nathalie Cartier. Adenoassociated virus-based gene therapy for cns diseases. Human gene therapy, 27(7):478-496, 2016. 
Daniel B Hoffmann, Kai O Böker, Stefan Schneider, Ellen Eckermann-Felkl, Angelina Schuder, Marina Komrakova, Stephan Sehmisch, and Jens Gruber. In vivo sirna delivery using jc virus-like particles decreases the expression of rankl in rats. Molecular Therapy-Nucleic Acids, 5:e298, 2016.

M David Hoggan, Neil R Blacklow, and WP Rowe. Studies of small dna viruses found in various adenovirus preparations: physical, biological, and immunological characteristics. Proceedings of the National Academy of Sciences, 55(6):1467-1474, 1966.

MD Hoggan, GF Thomas, FB Thomas, and FB Johnson. Continuous "carriage" of adenovirus associated virus genome in cell cultures in the absence of helper adenoviruses. In Proceedings of the fourth Lepetit colloquium, Cocoyac, Mexico, pages 243-249, 1972.

Douglas B Howard, Kathleen Powers, Yun Wang, and Brandon K Harvey. Tropism and toxicity of adenoassociated viral vector serotypes $1,2,5,6,7,8$, and 9 in rat neurons and glia in vitro. Virology, 372 (1):24-34, 2008.

Eloise Hudry, Courtney Martin, Sheetal Gandhi, Bence György, Deborah I Scheffer, Dakai Mu, Steven F Merkel, Federico Mingozzi, Zachary Fitzpatrick, Hemi Dimant, et al. Exosome-associated aav vector as a robust and convenient neuroscience tool. Gene therapy, 23(4):380-392, 2016.

Janina Huer. Top-down attention: neural pathways in the human and non-human primate examined by electrophysiology, optogenetics and psychophysics. Georg-August University Göttingen, 2018.

Tyler Janovitz, Thiago Oliveira, Michel Sadelain, and Erik Falck-Pedersen. Highly divergent integration profile of adeno-associated virus serotype 5 revealed by high-throughput sequencing. Journal of virology, 88(5):2481-2488, 2014.

Christopher Janson, Scott McPhee, Larissa Bilaniuk, John Haselgrove, Mark Testaiuti, Andrew Freese, Dah-Jyuu Wang, David Shera, Peter Hurh, Joan Rupin, et al. Gene therapy of canavan disease: Aav2 vector for neurosurgical delivery of aspartoacylase gene (aspa) to the human brain. Human gene therapy, 13(11):1391-1412, 2002.

Hayate Javed, Sindhu A Menon, Karima M Al-Mansoori, Abdelmojib Al-Wandi, Nour K Majbour, Mustafa T Ardah, Shiji Varghese, Nishant N Vaikath, M Emdadul Haque, Mimoun Azzouz, et al. Development of nonviral vectors targeting the brain as a therapeutic approach for parkinson's disease and other brain disorders. Molecular Therapy, 24(4):746-758, 2016.

Luna S Joffe, Leonardo Nimrichter, Marcio L Rodrigues, and Maurizio Del Poeta. Potential roles of fungal extracellular vesicles during infection. mSphere, 1(4):e00099-16, 2016.

F Brent Johnson, Harvey L Ozer, and M David Hoggan. Structural proteins of adenovirus-associated virus type 3. Journal of virology, 8(6):860-863, 1971.

Raghu Kalluri and Valerie S LeBleu. Discovery of double-stranded genomic dna in circulating exosomes. In Cold Spring Harbor Symposia on Quantitative Biology, volume 81, pages 275-280. Cold Spring Harbor Laboratory Press, 2016. 
Nikola Kaludov, Kevin E Brown, Robert W Walters, Joseph Zabner, and John A Chiorini. Adenoassociated virus serotype 4 (aav4) and aav5 both require sialic acid binding for hemagglutination and efficient transduction but differ in sialic acid linkage specificity. Journal of virology, 75(15):6884-6893, 2001.

Mark A Kay. State-of-the-art gene-based therapies: the road ahead. Nature Reviews Genetics, 12(5), 2011.

Arash Kianianmomeni and Armin Hallmann. Spotlighted brains: Optogenetic activation and silencing of neurons. Trends in biochemical sciences, 40(11):624-627, 2015.

V Narry Kim, Kyriacos Mitrophanous, Susan M Kingsman, and Alan J Kingsman. Minimal requirement for a lentivirus vector based on human immunodeficiency virus type 1. Journal of virology, 72(1): 811-816, 1998.

R Kirnbauer, F Booy, N Cheng, DR Lowy, and JT Schiller. Papillomavirus 11 major capsid protein selfassembles into virus-like particles that are highly immunogenic. Proceedings of the National Academy of Sciences, 89(24):12180-12184, 1992.

Sonja Kleinlogel. 23 optogenetics for vision recovery: From traditional to designer optogenetic tools. Optogenetics: From Neuronal Function to Mapping and Disease Biology, page 337, 2017.

Masanori Kobayashi, Akihiro Iida, Yasuji Ueda, and Mamoru Hasegawa. Pseudotyped lentivirus vectors derived from simian immunodeficiency virus sivagm with envelope glycoproteins from paramyxovirus. Journal of virology, 77(4):2607-2614, 2003.

Rika Komagome, Hirofumi Sawa, Takashi Suzuki, Yasuo Suzuki, Shinya Tanaka, Walter J Atwood, and Kazuo Nagashima. Oligosaccharides as receptors for jc virus. Journal of virology, 76(24):12992-13000, 2002.

Robert M Kotin, Marcello Siniscalco, R Jude Samulski, XD Zhu, Lynne Hunter, Catherine A Laughlin, Susan McLaughlin, Nicholas Muzyczka, Marino Rocchi, and Kenneth I Berns. Site-specific integration by adeno-associated virus. Proceedings of the National Academy of Sciences, 87(6):2211-2215, 1990.

Jane Kovalevich and Dianne Langford. Considerations for the use of sh-sy5y neuroblastoma cells in neurobiology. Neuronal Cell Culture: Methods and Protocols, pages 9-21, 2013.

Arthur M Krieg. Direct immunologic activities of cpg dna and implications for gene therapy. The journal of gene medicine, 1(1):56-63, 1999.

Robert H Kutner, Xian-Yang Zhang, and Jakob Reiser. Production, concentration and titration of pseudotyped hiv-1-based lentiviral vectors. Nature protocols, 4(4):495, 2009.

Madeline A Lancaster, Magdalena Renner, Carol-Anne Martin, Daniel Wenzel, Louise S Bicknell, Matthew E Hurles, Tessa Homfray, Josef M Penninger, Andrew P Jackson, and Juergen A Knoblich. Cerebral organoids model human brain development and microcephaly. Nature, 501(7467):373-379, 2013. 
LE Larsen, IF Heglund, R Fabian, P Walday, and WF Blazak. Neural tolerability of iodixanol in mice and dogs after single and repeated intracisternal administration. Acta Radiologica, 36(399_suppl): 238-243, 1995.

Thomas L Lentz. Rabies virus binding to an acetylcholine receptor $\alpha$-subunit peptide. Journal of Molecular Recognition, 3(2):82-88, 1990.

Bruce L Levine, Laurent M Humeau, Jean Boyer, Rob-Roy MacGregor, Tessio Rebello, Xiaobin Lu, Gwendolyn K Binder, Vladimir Slepushkin, Franck Lemiale, John R Mascola, et al. Gene transfer in humans using a conditionally replicating lentiviral vector. Proceedings of the National Academy of Sciences, 103(46):17372-17377, 2006.

Lian Li, Toshiharu Suzuki, Nozomu Mori, and Paul Greengard. Identification of a functional silencer element involved in neuron-specific expression of the synapsin i gene. Proceedings of the National Academy of Sciences, 90(4):1460-1464, 1993.

Diana H Lim, Jeffrey LeDue, Majid H Mohajerani, Matthieu P Vanni, and Timothy H Murphy. Optogenetic approaches for functional mouse brain mapping. Frontiers in neuroscience, 7, 2013.

Andrew J Link, Jimmy Eng, David M Schieltz, Edwin Carmack, Gregory J Mize, David R Morris, Barbara M Garvik, and John R Yates. Direct analysis of protein complexes using mass spectrometry. Nature biotechnology, 17(7):676-682, 1999.

Christine K Liu, Grant Wei, and Walter J Atwood. Infection of glial cells by the human polyomavirus jc is mediated by an n-linked glycoprotein containing terminal $\alpha(2-6)$-linked sialic acids. Journal of virology, 72(6):4643-4649, 1998.

Ying Poi Liu and Ben Berkhout. Hiv-1-based lentiviral vectors. Human Retroviruses: Methods and Protocols, pages 273-284, 2014.

Yuhe Liu, Takashi Okada, Tatsuya Nomoto, Xiaomei Ke, Akihiro Kume, Keiya Ozawa, and Shuifang Xiao. Promoter effects of adeno-associated viral vector for transgene expression in the cochlea in vivo. Experimental $\mathcal{E}$ molecular medicine, 39(2):170-175, 2007.

Andrea Maddalena, Patrizia Tornabene, Paola Tiberi, Renato Minopoli, Anna Manfredi, Margherita Mutarelli, Settimio Rossi, Francesca Simonelli, Jurgen K Naggert, Davide Cacchiarelli, et al. Triple vectors expand aav transfer capacity in the retina. Molecular Therapy, 2017.

Casey A Maguire, Leonora Balaj, Sarada Sivaraman, Matheus HW Crommentuijn, Maria Ericsson, Lucia Mincheva-Nilsson, Vladimir Baranov, Davide Gianni, Bakhos A Tannous, Miguel Sena-Esteves, et al. Microvesicle-associated aav vector as a novel gene delivery system. Molecular Therapy, 20(5):960-971, 2012.

Mark Mayford, Danny Baranes, Katrina Podsypanina, and Eric R Kandel. The 3'-untranslated region of camkii $\alpha$ is a cis-acting signal for the localization and translation of mrna in dendrites. Proceedings of the National Academy of Sciences, 93(23):13250-13255, 1996. 
DM McCarty, H Fu, PE Monahan, CE Toulson, P Naik, and RJ Samulski. Adeno-associated virus terminal repeat (tr) mutant generates self-complementary vectors to overcome the rate-limiting step to transduction in vivo. Gene therapy, 10(26):2112-2118, 2003.

Douglas M McCarty, Samuel M Young Jr, and R Jude Samulski. Integration of adeno-associated virus (aav) and recombinant aav vectors. Annu. Rev. Genet., 38:819-845, 2004.

ME McClements and RE MacLaren. Microrna-regulated viral vectors for gene therapy. Yale J Biol Med, 90(4):611-623, 2017.

Daniela Melchiorri, Luca Pani, Paolo Gasparini, Giulio Cossu, Janis Ancans, John Joseph Borg, Catherine Drai, Piotr Fiedor, Egbert Flory, Ian Hudson, et al. Regulatory evaluation of glybera in europe [mdash] two committees, one mission. Nature reviews Drug discovery, 12(9):719-719, 2013.

Amine Meliani, Florence Boisgerault, Zachary Fitzpatrick, Solenne Marmier, Christian Leborgne, Fanny Collaud, Marcelo Simon Sola, Severine Charles, Giuseppe Ronzitti, Alban Vignaud, et al. Enhanced liver gene transfer and evasion of preexisting humoral immunity with exosome-enveloped aav vectors. Blood advances, 1(23):2019-2031, 2017.

FJM Mergulhao, David K Summers, and Gabriel A Monteiro. Recombinant protein secretion in escherichia coli. Biotechnology advances, 23(3):177-202, 2005.

Agneta Mewes, Heike Franke, and David Singer. Organotypic brain slice cultures of adult transgenic p301s mice - a model for tauopathy studies. PloS one, 7(9):e45017, 2012.

Federico Mingozzi and Katherine A High. Immune responses to aav vectors: overcoming barriers to successful gene therapy. Blood, 122(1):23-36, 2013.

Hideki Mochizuki, Joan P Schwartz, Koichi Tanaka, Roscoe O Brady, and Jakob Reiser. High-titer human immunodeficiency virus type 1-based vector systems for gene delivery into nondividing cells. Journal of virology, 72(11):8873-8883, 1998.

Mozhdeh Mohammadian, Elham Abasi, and Abolfazl Akbarzadeh. Mesenchymal stem cell-based gene therapy: A promising therapeutic strategy. Artificial cells, nanomedicine, and biotechnology, 44(5): 1206-1211, 2016.

Robert B Moreland, Lynne Montross, and Robert L Garcea. Characterization of the dna-binding properties of the polyomavirus capsid protein vp1. Journal of virology, 65(3):1168-1176, 1991.

Tobias Moser. Optogenetic stimulation of the auditory pathway for research and future prosthetics. Current opinion in neurobiology, 34:29-36, 2015.

Shigeo Nagashima, Suljid Jirintai, Masaharu Takahashi, Tominari Kobayashi, Tsutomu Nishizawa, Tom Kouki, Takashi Yashiro, Hiroaki Okamoto, et al. Hepatitis e virus egress depends on the exosomal pathway, with secretory exosomes derived from multivesicular bodies. Journal of General Virology, 95 (10):2166-2175, 2014. 
Shigeo Nagashima, Masaharu Takahashi, Tominari Kobayashi, Tsutomu Nishizawa, Takashi Nishiyama, Putu Prathiwi Primadharsini, Hiroaki Okamoto, et al. Characterization of the quasi-enveloped hepatitis e virus particles released by the cellular exosomal pathway. Journal of Virology, 91(22):e00822-17, 2017.

Georg Nagel, Tanjef Szellas, Wolfram Huhn, Suneel Kateriya, Nona Adeishvili, Peter Berthold, Doris Ollig, Peter Hegemann, and Ernst Bamberg. Channelrhodopsin-2, a directly light-gated cation-selective membrane channel. Proceedings of the National Academy of Sciences, 100(24):13940-13945, 2003.

Luigi Naldini and Inder M Verma. Lentiviral vectors. Advances in virus research, 55:599-609, 2000.

Luigi Naldini, Ulrike Blomer, Philippe Gallay, Daniel Ory, Richard Mulligan, Fred H Gage, Inder M Verma, Didier Trono, et al. In vivo gene delivery and stable transduction of nondividing cells by a lentiviral vector. Science-AAAS-Weekly Paper Edition, 272(5259):263-267, 1996.

Shelley A Nass, Maryellen A Mattingly, Denise A Woodcock, Brenda L Burnham, Jeffrey A Ardinger, Shayla E Osmond, Amy M Frederick, Abraham Scaria, Seng H Cheng, and Catherine R O'Riordan. Universal method for the purification of recombinant aav vectors of differing serotypes. Molecular Therapy-Methods \& Clinical Development, 2017.

Ursula Neu, Melissa S Maginnis, Angelina S Palma, Luisa J Ströh, Christian DS Nelson, Ten Feizi, Walter J Atwood, and Thilo Stehle. Structure-function analysis of the human jc polyomavirus establishes the lstc pentasaccharide as a functional receptor motif. Cell host \& microbe, 8(4):309-319, 2010.

Tomas Olsson, Anat Achiron, Lars Alfredsson, Thomas Berger, David Brassat, Andrew Chan, Giancarlo Comi, Mefkure Eraksoy, Harald Hegen, Jan Hillert, et al. Anti-jc virus antibody prevalence in a multinational multiple sclerosis cohort. Multiple Sclerosis Journal, 19(11):1533-1538, 2013.

Harry O Orlans, Thomas L Edwards, Samantha R De Silva, Maria I Patrício, and Robert E MacLaren. Human retinal explant culture for ex vivo validation of aav gene therapy. In Retinal Gene Therapy, pages 289-303. Springer, 2018.

Wei-Chih Ou, Meilin Wang, CY Fung, RT Tsai, PC Chao, TH Hseu, and Deching Chang. The major capsid protein, vp1, of human jc virus expressed in escherichia coli is able to self-assemble into a capsid-like particle and deliver exogenous dna into human kidney cells. Journal of general virology, 80 (1):39-46, 1999.

David G Ousterout, Pablo Perez-Pinera, Pratiksha I Thakore, Ami M Kabadi, Matthew T Brown, Xiaoxia Qin, Olivier Fedrigo, Vincent Mouly, Jacques P Tremblay, and Charles A Gersbach. Reading frame correction by targeted genome editing restores dystrophin expression in cells from duchenne muscular dystrophy patients. Molecular Therapy, 21(9):1718-1726, 2013.

Adam M Packer, Darcy S Peterka, Jan J Hirtz, Rohit Prakash, Karl Deisseroth, and Rafael Yuste. Two-photon optogenetics of dendritic spines and neural circuits. Nature methods, 9(12):1202-1205, 2012. 
BillieL Padgett, GabrieleM Zurhein, DuardL Walker, RobertJ Eckroade, and BertH Dessel. Cultivation of papova-like virus from human brain with progressive multifocal leucoencephalopathy. The Lancet, 297(7712):1257-1260, 1971.

Stéphane Palfi, Jean Marc Gurruchaga, G Scott Ralph, Helene Lepetit, Sonia Lavisse, Philip C Buttery, Colin Watts, James Miskin, Michelle Kelleher, Sarah Deeley, et al. Long-term safety and tolerability of prosavin, a lentiviral vector-based gene therapy for parkinson's disease: a dose escalation, open-label, phase 1/2 trial. The Lancet, 383(9923):1138-1146, 2014.

Nicole K Paulk, Katja Pekrun, Erhua Zhu, Sean Nygaard, Bin Li, Jianpeng Xu, Kirk Chu, Christian Leborgne, Allison P Dane, Annelise Haft, et al. Bioengineered aav capsids with combined high human liver transduction in vivo and unique humoral seroreactivity. Molecular Therapy, 2017.

Dheeraj Pelluru, Roda Rani Konadhode, Narayan R Bhat, and Priyattam J Shiromani. Optogenetic stimulation of astrocytes in the posterior hypothalamus increases sleep at night in c57bl/6j mice. European Journal of Neuroscience, 43(10):1298-1306, 2016.

MT Pho, A Ashok, and Walter J Atwood. Jc virus enters human glial cells by clathrin-dependent receptor-mediated endocytosis. Journal of virology, 74(5):2288-2292, 2000.

Francoise Piguet, Sandro Alves, and Nathalie Cartier. Clinical gene therapy for neurodegenerative diseases: past, present, and future. Human gene therapy, 28(11):988-1003, 2017.

S Pillay, NL Meyer, AS Puschnik, O Davulcu, J Diep, Yet al Ishikawa, LT Jae, JE Wosen, CM Nagamine, MS Chapman, et al. An essential receptor for adeno-associated virus infection. Nature, 530(7588): 108-112, 2016.

Selvarangan Ponnazhagan, Gandham Mahendra, Sanjay Kumar, John A Thompson, and Mark Castillas Jr. Conjugate-based targeting of recombinant adeno-associated virus type 2 vectors by using avidin-linked ligands. Journal of virology, 76(24):12900-12907, 2002.

Sara Kathleen Powell, Ricardo Rivera-Soto, and Steven James Gray. Viral expression cassette elements to enhance transgene target specificity and expression in gene therapy. Discovery medicine, 19(102): 49, 2015.

Qiumin Qu, Hirofumi Sawa, Tadaki Suzuki, Shingo Semba, Chizuka Henmi, Yuki Okada, Masumi Tsuda, Shinya Tanaka, Walter J Atwood, and Kazuo Nagashima. Nuclear entry mechanism of the human polyomavirus jc virus-like particle role of importins and the nuclear pore complex. Journal of Biological Chemistry, 279(26):27735-27742, 2004.

W Querbes, BA O'hara, G Williams, and WJ Atwood. Invasion of host cells by jc virus identifies a novel role for caveolae in endosomal sorting of noncaveolar ligands. Journal of virology, 80(19):9402-9413, 2006 .

William Querbes, Alexandre Benmerah, Daniela Tosoni, PP Di Fiore, and Walter J Atwood. A jc virusinduced signal is required for infection of glial cells by a clathrin-and eps15-dependent pathway. Journal of virology, 78(1):250-256, 2004. 
Peter M Quinn, Thilo M Buck, Charlotte Ohonin, Harald MM Mikkers, and Jan Wijnholds. Production of ips-derived human retinal organoids for use in transgene expression assays. In Retinal Gene Therapy, pages 261-273. Springer, 2018.

Ulrich-Peter Rohr, Marc-Andre Wulf, Susanne Stahn, Ulrich Steidl, Rainer Haas, and Ralf Kronenwett. Fast and reliable titration of recombinant adeno-associated virus type-2 using quantitative real-time pcr. Journal of virological methods, 106(1):81-88, 2002.

Asya Rolls, Damien Colas, Antoine Adamantidis, Matt Carter, Tope Lanre-Amos, H Craig Heller, and Luis de Lecea. Optogenetic disruption of sleep continuity impairs memory consolidation. Proceedings of the National Academy of Sciences, 108(32):13305-13310, 2011.

Steven A Rosenberg, Paul Aebersold, Kenneth Cornetta, Attan Kasid, Richard A Morgan, Robert Moen, Evelyn M Karson, Michael T Lotze, James C Yang, Suzanne L Topalian, et al. Gene transfer into humans-immunotherapy of patients with advanced melanoma, using tumor-infiltrating lymphocytes modified by retroviral gene transduction. New England Journal of Medicine, 323(9):570-578, 1990.

David W Russell and Roll K Hirata. Human gene targeting by viral vectors. Nature genetics, 18(4): 325-330, 1998.

Dyana T Saenz, Román Barraza, Nils Loewen, Wulin Teo, and Eric M Poeschla. Feline immunodeficiency virus-based lentiviral vectors. Cold Spring Harbor Protocols, 2012(1):pdb-ip067579, 2012.

Dinakar M Salunke, Donald LD Caspar, and Robert L Garcea. Self-assembly of purified polyomavirus capsid protein vp1. Cell, 46(6):895-904, 1986.

RJ Samulski, X Zhu, X Xiao, JD Brook, DE Housman, Net al Epstein, and LA Hunter. Targeted integration of adeno-associated virus (aav) into human chromosome 19. The EMBO journal, 10(12): 3941, 1991.

Hong San, Zhi-Yong Yang, Vincent J Pompili, Michele L Jaffe, Gregory E Plautz, Ling Xu, Jiin H Felgner, Carl J Wheeler, Philip L Felgner, Xiang Gao, et al. Safety and short-term toxicity of a novel cationic lipid formulation for human gene therapy. Human gene therapy, 4(6):781-788, 1993.

Arpiar Saunders, Caroline A Johnson, and Bernardo L Sabatini. Novel recombinant adeno-associated viruses for cre activated and inactivated transgene expression in neurons. Frontiers in neural circuits, $6,2012$.

Frank Schakowski, Marcus Gorschlüter, Claas Junghans, Matthias Schroff, Peter Buttgereit, Carsten Ziske, Björn Schöttker, Sven A König-Merediz, Tilman Sauerbruch, Burghardt Wittig, et al. A novel minimal-size vector (midge) improves transgene expression in colon carcinoma cells and avoids transfection of undesired dna. Molecular Therapy, 3(5):793-800, 2001.

Catherine H Schein and Mathieu HM Noteborn. Formation of soluble recombinant proteins in escherichia coli is favored by lower growth temperature. Nature Biotechnology, 6(3):291-294, 1988.

Lara Timantra Schiller, Nicolás Lemus-Diaz, Rafael Rinaldi Ferreira, Kai Oliver Böker, and Jens Gruber. Enhanced production of exosome-associated aav by overexpression of the tetraspanin cd9. Molecular Therapy-Methods \& Clinical Development, 9:278-287, 2018. 
Ryan Schlimgen, John Howard, Dawn Wooley, Maureen Thompson, Lindsey R Baden, Otto O Yang, David C Christiani, Gustavo Mostoslavsky, David V Diamond, Elizabeth Gilman Duane, et al. Risks associated with lentiviral vector exposures and prevention strategies. Journal of occupational and environmental medicine, 58(12):1159, 2016.

Z Shevtsova, JMI Malik, U Michel, M Bähr, and S Kügler. Promoters and serotypes: targeting of adeno-associated virus vectors for gene transfer in the rat central nervous system in vitro and in vivo. Experimental physiology, 90(1):53-59, 2005.

Yutaka Shimada, DA Fischman, and AA Moscona. Formation of neuromuscular junctions in embryonic cell cultures. Proceedings of the National Academy of Sciences, 62(3):715-721, 1969.

Richard H Smith, Chuantian Ding, and Robert M Kotin. Serum-free production and column purification of adeno-associated virus type 5. Journal of virological methods, 114(2):115-124, 2003.

M Stahlberg. Nanoscale probing of single synapse function and bdnf cell-to-cell transfer. Dissertation, Georg-August University Göttingen, 2016.

Lisa M Stanek, Sergio P Sardi, Bryan Mastis, Amy R Richards, Christopher M Treleaven, Tatyana Taksir, Kuma Misra, Seng H Cheng, and Lamya S Shihabuddin. Silencing mutant huntingtin by adenoassociated virus-mediated rna interference ameliorates disease manifestations in the yac128 mouse model of huntington's disease. Human gene therapy, 25(5):461-474, 2014.

Stefan Stender, Mary Murphy, Tim O'Brien, Carsten Stengaard, Michael Ulrich-Vinther, Kjeld Soballe, and Frank Barry. Adeno-associated viral vector transduction of human mesenchymal stem cells. Eur Cell Mater, 13(93-99):99, 2007.

Benjamin Strobel, Felix D Miller, Wolfgang Rist, and Thorsten Lamla. Comparative analysis of cesium chloride-and iodixanol-based purification of recombinant adeno-associated viral vectors for preclinical applications. Human gene therapy methods, 26(4):147-157, 2015.

Satoko Suzuki, Hirofumi Sawa, Rika Komagome, Yasuko Orba, Misato Yamada, Yuki Okada, Yusuke Ishida, Hiroshi Nishihara, Shinya Tanaka, and Kazuo Nagashima. Broad distribution of the jc virus receptor contrasts with a marked cellular restriction of virus replication. Virology, 286(1):100-112, 2001.

Jean-Marc Taymans, Luk H Vandenberghe, Chris Van Den Haute, Irina Thiry, Christophe M Deroose, Luc Mortelmans, James M Wilson, Zeger Debyser, and Veerle Baekelandt. Comparative analysis of adeno-associated viral vector serotypes $1,2,5,7$, and 8 in mouse brain. Human gene therapy, 18(3): 195-206, 2007.

Karin Tegerstedt, Andrea Vlastos Franzén, Kalle Andreasson, Jeanna Joneberg, Shirin Heidari, Ternbörn Ramgvist, and Tina Dalianis. Murine polyomavirus virus-like particles (vlps) as vectors for gene and immune therapy and vaccines against viral infections and cancer. Anticancer research, 25(4):2601-2608, 2005. 
Liliane Tenenbaum, Malika Hamdane, Marianne Pouzet, Bernard Avalosse, A Stathopoulos, Fabrice Jurysta, C Rosenbaum, CO Hanemann, Marc Levivier, and Thierry Velu. Cellular contaminants of adeno-associated virus vector stocks can enhance transduction. Gene therapy, 6(6), 1999.

Clotilde Théry, Laurence Zitvogel, and Sebastian Amigorena. Exosomes: composition, biogenesis and function. Nature Reviews Immunology, 2(8), 2002.

Brian G Till, Michael C Jensen, Jinjuan Wang, Eric Y Chen, Brent L Wood, Harvey A Greisman, Xiaojun Qian, Scott E James, Andrew Raubitschek, Stephen J Forman, et al. Adoptive immunotherapy for indolent non-hodgkin lymphoma and mantle cell lymphoma using genetically modified autologous cd20-specific t cells. Blood, 112(6):2261-2271, 2008.

Hélio A Tomás, Ana F Rodrigues, Paula M Alves, and Ana S Coroadinha. Lentiviral gene therapy vectors: challenges and future directions. In Gene Therapy-Tools and Potential Applications. InTech, 2013.

VFI Van Tendeloo, R Willems, P Ponsaerts, M Lenjou, G Nijs, M Vanhove, P Muylaert, P Van Cauwelaert, C Van Broeckhoven, DR Van Bockstaele, et al. High-level transgene expression in primary human t lymphocytes and adult bone marrow cd34+ cells via electroporation-mediated gene delivery. Gene therapy, $7(16): 1431,2000$.

Luk H Vandenberghe, Ru Xiao, Martin Lock, Jianping Lin, Michael Korn, and James M Wilson. Efficient serotype-dependent release of functional vector into the culture medium during adeno-associated virus manufacturing. Human gene therapy, 21(10):1251-1257, 2010.

Marc Vandevelde, Robert Higgins, and Anna Oevermann. Veterinary neuropathology: essentials of theory and practice. John Wiley \& Sons, 2012.

Robert W Walters, Su Min P Yi, Shaf Keshavjee, Kevin E Brown, Michael J Welsh, John A Chiorini, and Joseph Zabner. Binding of adeno-associated virus type 5 to 2, 3-linked sialic acid is required for gene transfer. Journal of Biological Chemistry, 276(23):20610-20616, 2001.

Robert W Walters, Mavis Agbandje-McKenna, Valorie D Bowman, Thomas O Moninger, Norman H Olson, Michael Seiler, John A Chiorini, Timothy S Baker, and Joseph Zabner. Structure of adenoassociated virus serotype 5. Journal of virology, 78(7):3361-3371, 2004.

Feng Wang, Xiuling Cui, Mingxi Wang, Yaqing Wu, Weidong Xiao, and Ruian Xu. A reliable and feasible qpcr strategy for titrating aav vectors. Medical science monitor basic research, 19:187, $2013 \mathrm{a}$.

Lili Wang, Joanita Figueredo, Roberto Calcedo, Jianping Lin, and James M Wilson. Cross-presentation of adeno-associated virus serotype 2 capsids activates cytotoxic $t$ cells but does not render hepatocytes effective cytolytic targets. Human gene therapy, 18(3):185-194, 2007.

Lizheng Wang, Zixuan Wang, Fangfang Zhang, Rui Zhu, Jinpeng Bi, Jiaxin Wu, Haihong Zhang, Hui Wu, Wei Kong, Bin Yu, et al. Enhancing transgene expression from recombinant aav8 vectors in different tissues using woodchuck hepatitis virus post-transcriptional regulatory element. International journal of medical sciences, 13(4):286, 2016. 
Qilong Wang, Xiaoying Zhuang, Jingyao Mu, Zhong-Bin Deng, Hong Jiang, Lifeng Zhang, Xiaoyu Xiang, Baomei Wang, Jun Yan, Donald Miller, et al. Delivery of therapeutic agents by nanoparticles made of grapefruit-derived lipids. Nature communications, 4:1867, 2013b.

Sarah J Wassmer, Livia S Carvalho, Bence György, Luk H Vandenberghe, and Casey A Maguire. Exosome-associated aav2 vector mediates robust gene delivery into the murine retina upon intravitreal injection. Scientific Reports, 7:45329, 2017.

Matthew D Weitzman, SR Kyöstiö, Robert M Kotin, and Roland A Owens. Adeno-associated virus (aav) rep proteins mediate complex formation between aav dna and its integration site in human dna. Proceedings of the National Academy of Sciences, 91(13):5808-5812, 1994.

Natalie Jayne Werling, Stifani Satkunanathan, Robin Thorpe, and Yuan Zhao. Systematic comparison and validation of quantitative real-time pcr methods for the quantitation of adeno-associated viral products. Human gene therapy methods, 26(3):82-92, 2015.

Martyn K White and Kamel Khalili. Pathogenesis of progressive multifocal leukoencephalopathy — revisited. Journal of Infectious Diseases, 203(5):578-586, 2011.

Martyn K White, Mahmut Safak, and Kamel Khalili. Regulation of gene expression in primate polyomaviruses. Journal of virology, 83(21):10846-10856, 2009.

William Wold and Karoly Toth. Adenovirus vectors for gene therapy, vaccination and cancer gene therapy. Current gene therapy, 13(6):421-433, 2013.

Hassen S Wollebo, Baheru Woldemichaele, Kamel Khalili, Mahmut Safak, and Martyn K White. Epigenetic regulation of polyomavirus jc. Virology journal, 10(1):264, 2013.

Liang-Fong Wong, Mimoun Azzouz, Lucy E Walmsley, Zoe Askham, Fraser J Wilkes, Kyriacos A Mitrophanous, Susan M Kingsman, and Nicholas D Mazarakis. Transduction patterns of pseudotyped lentiviral vectors in the nervous system. Molecular Therapy, 9(1):101-111, 2004.

Zhijian Wu, Aravind Asokan, and R Jude Samulski. Adeno-associated virus serotypes: vector toolkit for human gene therapy. Molecular therapy, 14(3):316-327, 2006.

Zhijian Wu, Hongyan Yang, and Peter Colosi. Effect of genome size on aav vector packaging. Molecular Therapy, 18(1):80-86, 2010.

Lin Yang, Juan Li, and Xiao Xiao. Directed evolution of adeno-associated virus (aav) as vector for muscle gene therapy. Muscle Gene Therapy: Methods and Protocols, pages 127-139, 2011.

Yae Jin Yoon, Oh Youn Kim, and Yong Song Gho. Extracellular vesicles as emerging intercellular communicasomes. BMB reports, 47(10):531, 2014.

Nadja Zeltner, Erik Kohlbrenner, Nathalie Clément, Thomas Weber, and R Michael Linden. Near-perfect infectivity of wild-type aav as benchmark for infectivity of recombinant aav vectors. Gene therapy, 17 (7):872, 2010. 
Feng Zhang, Viviana Gradinaru, Antoine R Adamantidis, Remy Durand, Raag D Airan, Luis De Lecea, and Karl Deisseroth. Optogenetic interrogation of neural circuits: technology for probing mammalian brain structures. Nature protocols, 5(3):439-456, 2010.

Yangbing Zhao, Zhili Zheng, Cyrille J Cohen, Luca Gattinoni, Douglas C Palmer, Nicholas P Restifo, Steven A Rosenberg, and Richard A Morgan. High-efficiency transfection of primary human and mouse t lymphocytes using rna electroporation. Molecular therapy, 13(1):151-159, 2006.

Carmela Zincarelli, Stephen Soltys, Giuseppe Rengo, and Joseph E Rabinowitz. Analysis of aav serotypes 1-9 mediated gene expression and tropism in mice after systemic injection. Molecular Therapy, 16(6): 1073-1080, 2008.

S Zolotukhin, BJ Byrne, E Mason, I Zolotukhin, M Potter, K Chesnut, C Summerford, RJ Samulski, and N Muzyczka. Recombinant adeno-associated virus purification using novel methods improves infectious titer and yield. Gene therapy, 6(6), 1999.

Romain Zufferey, Thomas Dull, Ronald J Mandel, Anatoly Bukovsky, Dulce Quiroz, Luigi Naldini, and Didier Trono. Self-inactivating lentivirus vector for safe and efficient in vivo gene delivery. Journal of virology, 72(12):9873-9880, 1998. 


\section{Appendix}

\subsection{Plasmid maps}

5.1.1 rAAV genetic toolbox
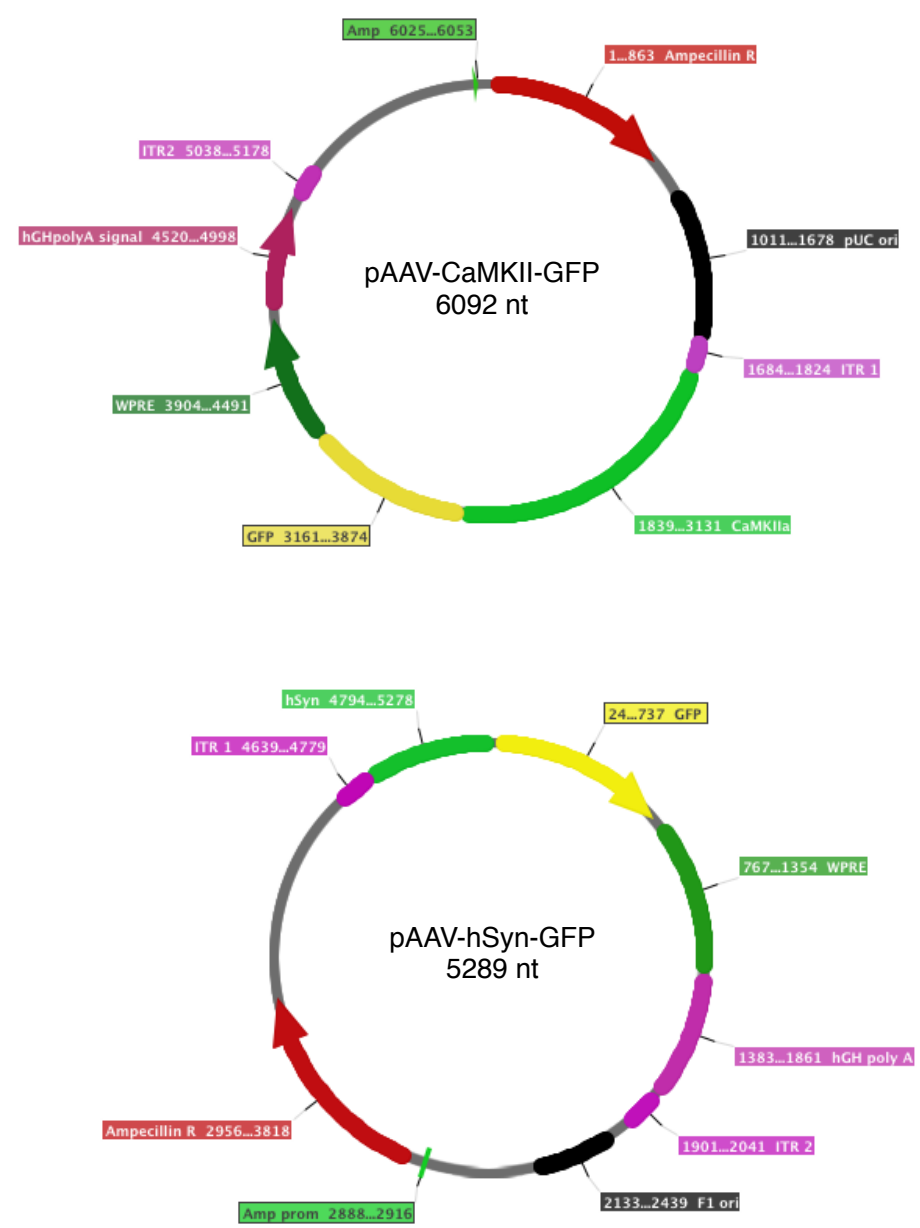

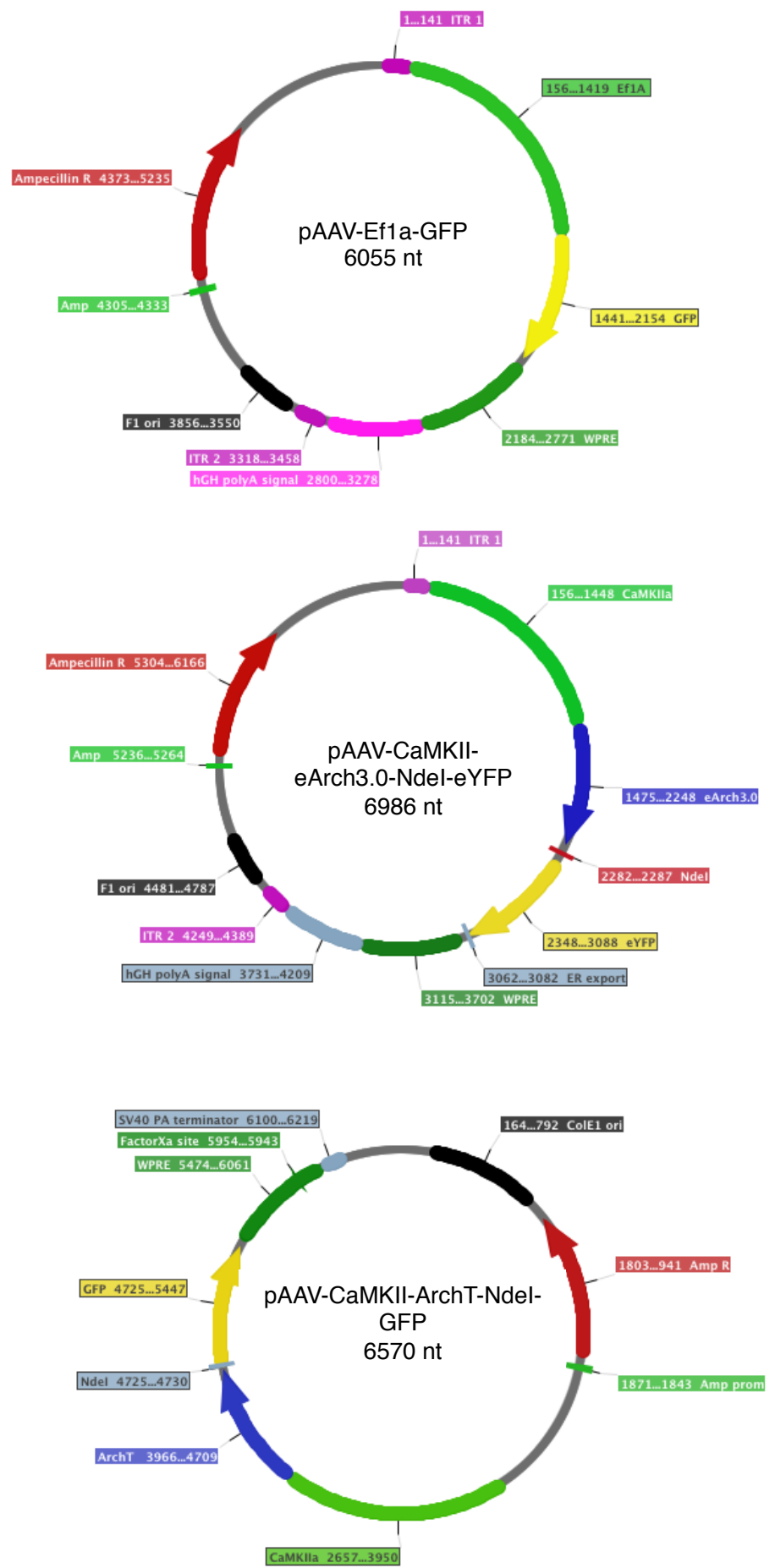

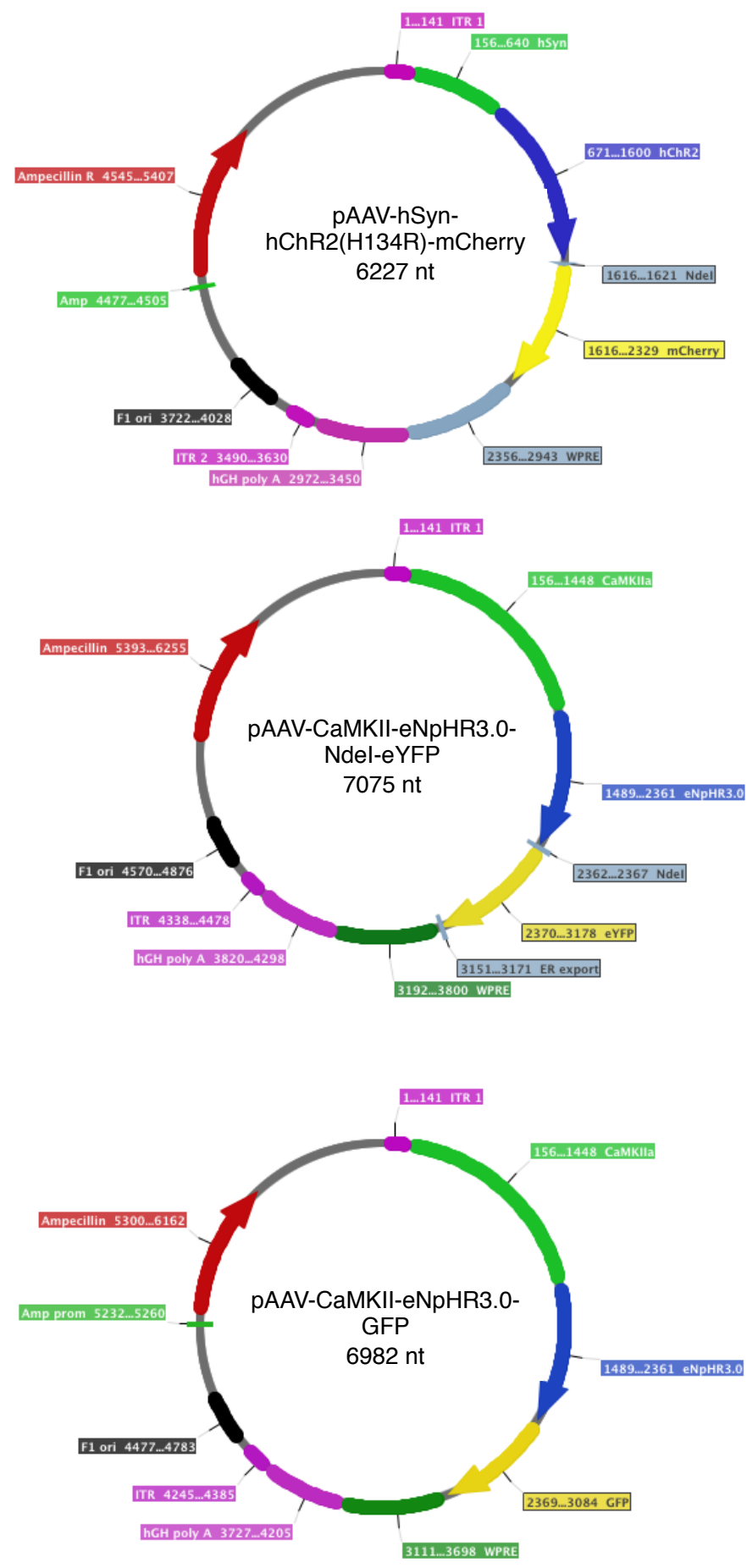

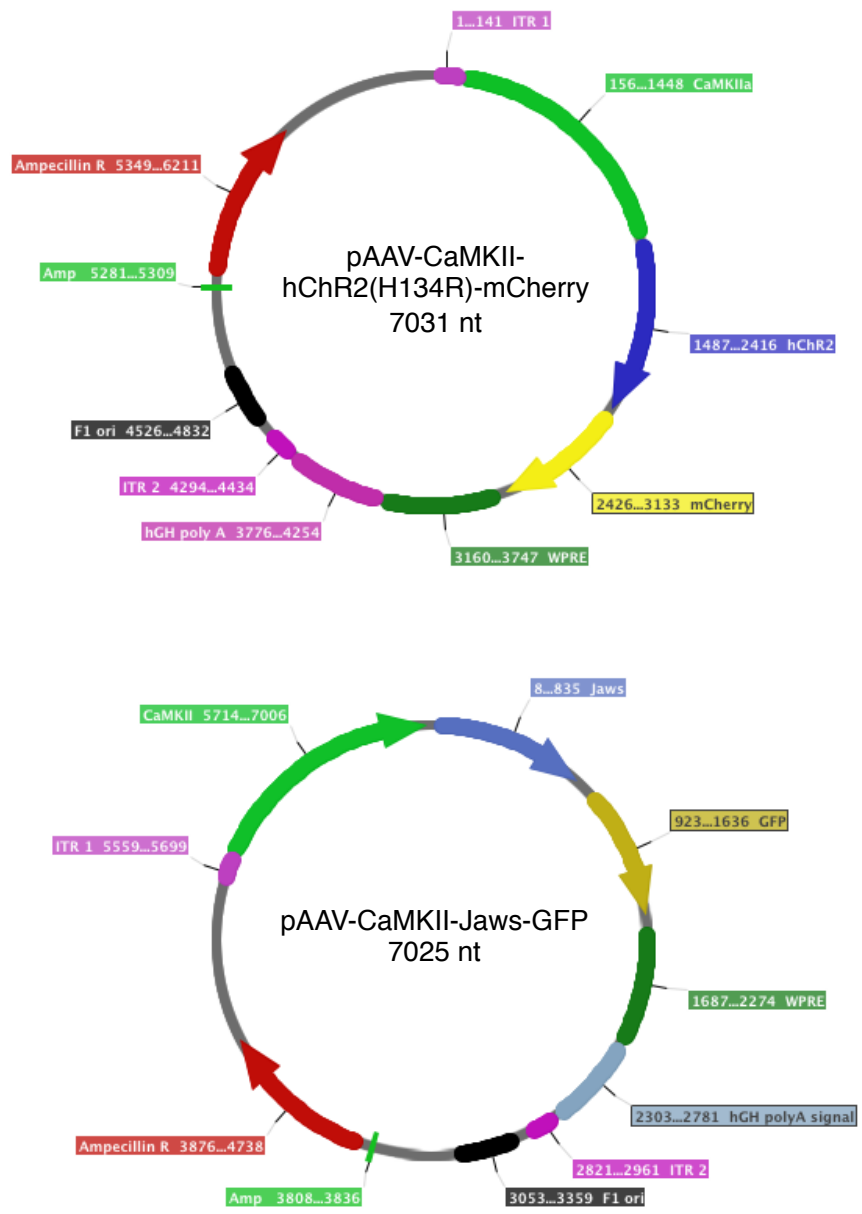


\subsubsection{Linear expression cassette plasmids}
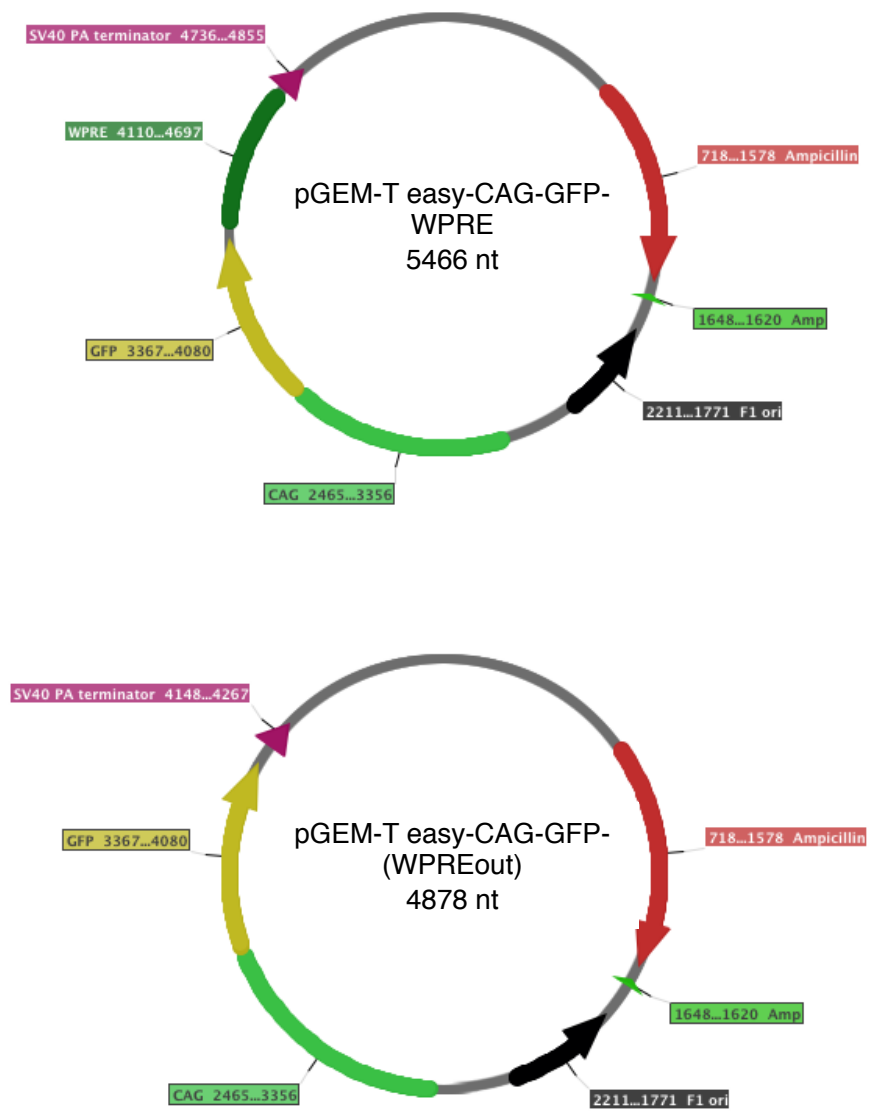


\subsection{3 scFv expression plasmids}
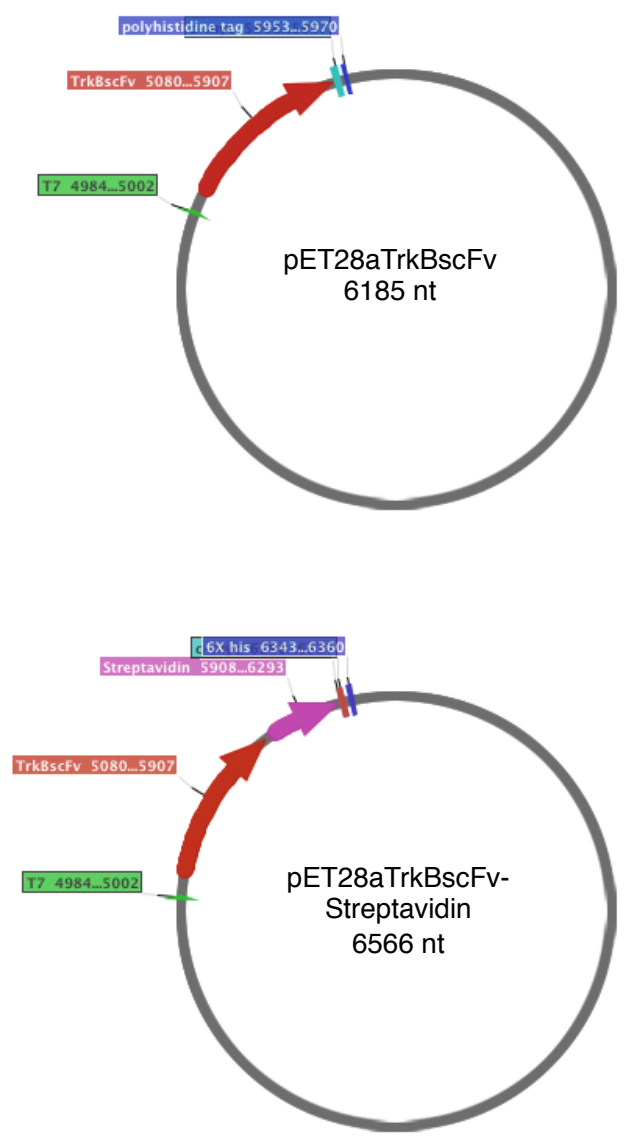


\subsection{Mass spectrometry analysis of impurity lanes}

Table 45: Mass spectrometry hits

\begin{tabular}{|c|c|c|c|}
\hline Number & Identified proteins & $\begin{array}{l}\text { Molecular } \\
\text { weight }\end{array}$ & $\begin{array}{l}\text { Total of } \\
\text { spectra }\end{array}$ \\
\hline 1 & Ferritin light chain (Homo sapiens) & $20 \mathrm{kDa}$ & $0.44 \%$ \\
\hline 2 & Trypsin (Sus scrofa) & $24 \mathrm{kDa}$ & $0.37 \%$ \\
\hline 3 & Ferritin heavy chain (Homo sapiens) & $21 \mathrm{kDa}$ & $0.33 \%$ \\
\hline 4 & Keratin type II cytoskeletal 1 (Homo sapiens) & $66 \mathrm{kDa}$ & $0.19 \%$ \\
\hline 5 & Keratin type II cytoskeletal 2 epidermal (Homo sapiens) & $65 \mathrm{kDa}$ & $0.13 \%$ \\
\hline 6 & Keratin type I cytoskeletal 10 (Homo sapiens) & $59 \mathrm{kDa}$ & $0.12 \%$ \\
\hline 7 & Keratin type I cytoskeletal 9 (Homo sapiens) & $62 \mathrm{kDa}$ & $0.089 \%$ \\
\hline 8 & Proteasome subunit beta type-5 (Homo sapiens) & $28 \mathrm{kDa}$ & $0.089 \%$ \\
\hline 9 & Ferritin light chain (Bos taurus) & $20 \mathrm{kDa}$ & $0.059 \%$ \\
\hline 10 & Tubulin beta-4 chain (Xenopus laevis) & $50 \mathrm{kDa}$ & $0.049 \%$ \\
\hline 11 & Elongation factor Tu 1 (Escherichia coli) & $43 \mathrm{kDa}$ & $0.05 \%$ \\
\hline 12 & Proteasome subunit alpha type-1 (Homo sapiens) & $30 \mathrm{kDa}$ & $0.04 \%$ \\
\hline 13 & ADP-ribosylation factor 5 (Homo sapiens) & $21 \mathrm{kDa}$ & $0.04 \%$ \\
\hline 14 & Proteasome subunit alpha type-5 (Bos taurus) & $26 \mathrm{kDa}$ & $0.04 \%$ \\
\hline 15 & Keratin, type I cytoskeletal 14 (Homo sapiens) & $52 \mathrm{kDa}$ & $0.03 \%$ \\
\hline 16 & Proteasome subunit beta type-7 (Homo sapiens) & $30 \mathrm{kDa}$ & $0.03 \%$ \\
\hline 17 & Tubulin alpha-1B chain (Bos taurus) & $50 \mathrm{kDa}$ & $0.03 \%$ \\
\hline 18 & Actin-1 (Aedes aegypti) & $42 \mathrm{kDa}$ & $0.03 \%$ \\
\hline 19 & 40S ribosomal protein S18 (Bos taurus) & $18 \mathrm{kDa}$ & $0.03 \%$ \\
\hline
\end{tabular}



vis)

E1B protein, small T-antigen (Human adenovirus C $21 \mathrm{kDa}$ serotype 2)

Ferritin heavy chain (Equus caballus)

$21 \mathrm{kDa} \quad 0.03 \%$

Proteasome subunit alpha type-7 (Bos taurus)

$28 \mathrm{kDa} \quad 0.02 \%$

60S acidic ribosomal protein P2 (Bos taurus)

$12 \mathrm{kDa}$

$0.02 \%$

Destrin (Bos taurus)

$19 \mathrm{kDa} \quad 0.02 \%$

60S ribosomal protein L12 (Bos taurus)

$18 \mathrm{kDa} \quad 0.02 \%$

Proteasome subunit beta type-1 (Homo sapiens)

$26 \mathrm{kDa} \quad 0.02 \%$

Green fluorescent protein (Aequorea victoria)

$27 \mathrm{kDa} \quad 0.02 \%$

Heat shock protein HSP 90-beta (Equus caballus)

$83 \mathrm{kDa} \quad 0.02 \%$

Glutamine synthetase (Homo sapiens)

$42 \mathrm{kDa} \quad 0.02 \%$

60S ribosomal protein L26 (Bos taurus)

$17 \mathrm{kDa} \quad 0.02 \%$

Ferritin heavy chain (Bos taurus)

$21 \mathrm{kDa}$

$0.02 \%$

Protein GlcG (Escherichia coli)

$14 \mathrm{kDa}$

$0.02 \%$

Eukaryotic translation initiation factor 5A-1 (Bos tau-

$17 \mathrm{kDa}$

$0.02 \%$ rus)

Maltose-binding periplasmic protein (Escherichia coli)

$43 \mathrm{kDa}$

$0.02 \%$

DNA-directed RNA polymerases I, II, and III subunit

$17 \mathrm{kDa}$ 
$20 \mathrm{kDa}$ (Homo sapiens) aethiops)

Small nuclear ribonucleoprotein-associated protein B'

$25 \mathrm{kDa}$

$0.02 \%$ (Bos taurus)

Transcription elongation factor B polypeptide 2 (Homo

$13 \mathrm{kDa}$ sapiens)

Cellular nucleic acid-binding protein (Bos taurus)

$19 \mathrm{kDa}$

$0.01 \%$

$27 \mathrm{kDa}$

$0.01 \%$

Proteasome subunit beta type-6 (Homo sapiens)

$25 \mathrm{kDa}$

$0.01 \%$

$16 \mathrm{kDa}$

$0.01 \%$

$20 \mathrm{kDa}$

$0.01 \%$

Proteasome subunit beta type-3 (Bos taurus)

$23 \mathrm{kDa}$

$0.01 \%$ 

dratase subunit beta (Rhodococcus rhodochrous) (Bos taurus)

High-molecular weight cobalt-containing nitrile hy

$23 \mathrm{kDa}$ dratase subunit alpha (Rhodococcus rhodochrous) 


\begin{tabular}{|c|c|c|c|}
\hline 86 & Proteasome subunit alpha type-3 (Bos taurus) & $28 \mathrm{kDa}$ & $0.00 \%$ \\
\hline 87 & 60 S ribosomal protein L22 (Gallus gallus) & $15 \mathrm{kDa}$ & $0.00 \%$ \\
\hline 88 & Adenine phosphoribosyltransferase (Homo sapiens) & $20 \mathrm{kDa}$ & $0.00 \%$ \\
\hline 89 & GTP-binding nuclear protein Ran (Bos taurus) & $24 \mathrm{kDa}$ & $0.00 \%$ \\
\hline 90 & Tubulin beta- 5 chain (Bos taurus) & $50 \mathrm{kDa}$ & $0.00 \%$ \\
\hline 91 & Keratin, type I cytoskeletal 17 (Homo sapiens) & $48 \mathrm{kDa}$ & $0.00 \%$ \\
\hline 92 & $26 \mathrm{~S}$ protease regulatory subunit 7 (Bos taurus) & $49 \mathrm{kDa}$ & $0.00 \%$ \\
\hline 93 & Creatine kinase B-type (Bos taurus) & $43 \mathrm{kDa}$ & $0.00 \%$ \\
\hline 94 & 40S ribosomal protein S13 (Bos taurus) & $17 \mathrm{kDa}$ & $0.00 \%$ \\
\hline 95 & Dermcidin (Homo sapiens) & $11 \mathrm{kDa}$ & $0.00 \%$ \\
\hline 96 & ADP-ribosylation factor-like protein 3 (Bos taurus) & $21 \mathrm{kDa}$ & $0.00 \%$ \\
\hline 97 & Phosphoglycerate kinase 1 (Equus caballus) & $45 \mathrm{kDa}$ & $0.00 \%$ \\
\hline 98 & $\begin{array}{l}\text { Signal recognition particle } 14 \mathrm{kDa} \text { protein (Pongo } \\
\text { abelii) }\end{array}$ & $15 \mathrm{kDa}$ & $0.00 \%$ \\
\hline 99 & $40 \mathrm{~S}$ ribosomal protein $\mathrm{SA}$ (Bos taurus) & $33 \mathrm{kDa}$ & $0.00 \%$ \\
\hline 100 & $\begin{array}{l}\text { Electron transfer flavoprotein subunit beta (Mycobac- } \\
\text { terium bovis) }\end{array}$ & $28 \mathrm{kDa}$ & $0.00 \%$ \\
\hline 101 & Ig kappa chain V-I region AG (Homo sapiens) & $12 \mathrm{kDa}$ & $0.00 \%$ \\
\hline 102 & $\begin{array}{l}\text { Mitotic spindle-associated MMXD complex subunit } \\
\text { MIP18 (Homo sapiens) }\end{array}$ & $18 \mathrm{kDa}$ & $0.00 \%$ \\
\hline 103 & $\begin{array}{l}\text { Peptidyl-prolyl cis-trans isomerase NIMA-interacting } 4 \\
\text { (Bos taurus) }\end{array}$ & $14 \mathrm{kDa}$ & $0.00 \%$ \\
\hline 104 & $60 \mathrm{~S}$ ribosomal protein L27a (Bos taurus) & $17 \mathrm{kDa}$ & $0.00 \%$ \\
\hline 105 & Thioredoxin-1 (Escherichia coli) & $12 \mathrm{kDa}$ & $0.00 \%$ \\
\hline 106 & Ubiquitin-conjugating enzyme E2 N (Bos taurus) & $17 \mathrm{kDa}$ & $0.00 \%$ \\
\hline 107 & Vesicle-associated membrane protein 2 (Bos taurus) & $13 \mathrm{kDa}$ & $0.00 \%$ \\
\hline
\end{tabular}


108

109

110

111

112

113

114

115

116
40S ribosomal protein S19 (Bos taurus)

NudC domain-containing protein 2 (Homo sapiens)

Proteasome subunit alpha type-2 (Bos taurus)

$60 \mathrm{~S}$ ribosomal protein L18a (Bos taurus)

Alpha-enolase (Homo sapiens)

Streptavidin-V1 (Streptomyces violaceus)

Thioredoxin (Homo sapiens)

Peroxiredoxin-2 (Cricetulus griseus)

60S ribosomal protein L23 (Bos taurus)

Protein Hikeshi (Bos taurus)

Splicing factor $3 \mathrm{~B}$ subunit 3 (Bos taurus)

Eukaryotic translation initiation factor chromosomal (Homo sapiens)

\begin{abstract}
Chromosomal (Homo sapiens)
\end{abstract}
Cancer-related nucleoside-triphosphatase (Homo sapi- 21 kDa ens)

Prefoldin subunit 2 (Bos taurus)

$17 \mathrm{kDa}$

$18 \mathrm{kDa}$

60S ribosomal protein L24 (Bos taurus)

$60 \mathrm{kDa}$ heat shock protein, mitochondrial (Bos taurus)

$61 \mathrm{kDa}$

$17 \mathrm{kDa}$

$15 \mathrm{kDa}$

$15 \mathrm{kDa}$

40S ribosomal protein S15a (Bos taurus)

$34 \mathrm{kDa}$

General transcription factor IIH subunit 3 (Bos taurus)

Clathrin light chain A (Bos taurus)

$27 \mathrm{kDa}$

Heterogeneous nuclear ribonucleoprotein A1-like $2 \quad 34 \mathrm{kDa}$ (Homo sapiens)
$0.00 \%$

$0.00 \%$

$0.00 \%$

$0.00 \%$

$0.00 \%$

$0.00 \%$

$0.00 \%$

$0.00 \%$

$0.00 \%$

$0.00 \%$

$0.00 \%$

$0.00 \%$

$0.00 \%$

$0.00 \%$

$0.00 \%$

$0.00 \%$

$0.00 \%$

$0.00 \%$

$0.00 \%$

$0.00 \%$

$0.00 \%$

$0.00 \%$ 
$130 \quad$ Four and a half LIM domains protein 1 (Homo sapiens) $\quad 36 \mathrm{kDa} \quad 0.00 \%$

131 Heterogeneous nuclear ribonucleoproteins A2/B1 (Bos $36 \mathrm{kDa} \quad 0.00 \%$ taurus)

$132 \quad 40 \mathrm{~S}$ ribosomal protein S23 (Bos taurus) $\quad 16 \mathrm{kDa} \quad 0.00 \%$

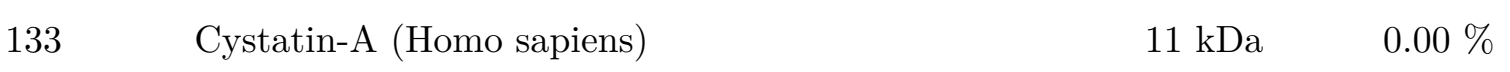

$134 \quad$ Ferritin heavy chain (Canis familiaris) $\quad 21 \mathrm{kDa} \quad 0.00 \%$

Proteins $<0.01 \%$ of total of spectra are labeled as $0.00 \%$. 


\section{3 rAAV5 injection in the cochlea of mice}
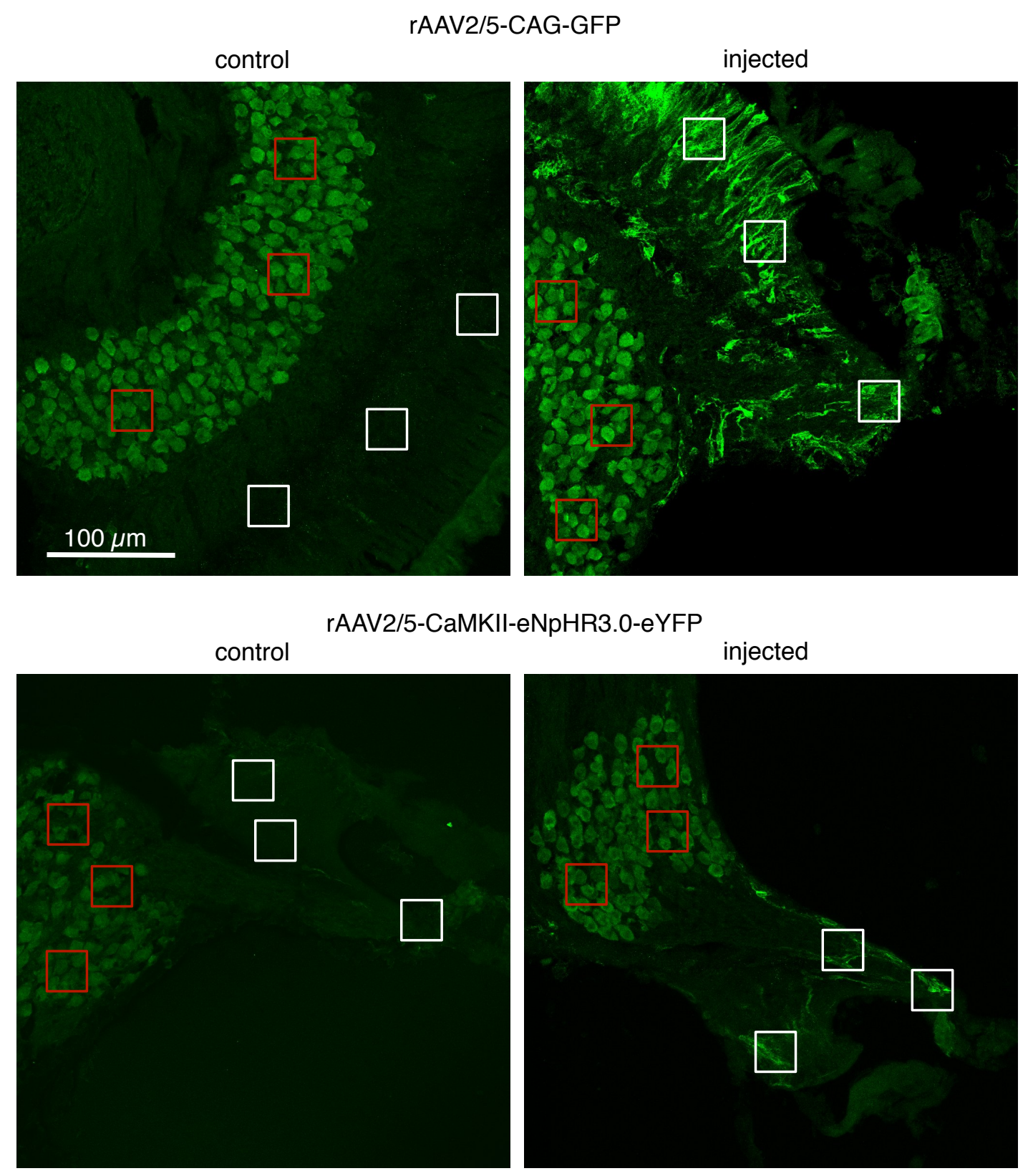

red $=$ spiral ganglion neurons

white $=$ supporting cells

Figure 34: Expression of rAAV5 in the mouse cochlea rAAV2/5-CAG-GFP and rAAV2/5-CaMKII-eNpHR3.0-eYFP were injected in the right cochleas of mice, and the left uninjected cochleas served as controls. The cochleas were subsequently stained against GFP. Relative GFP intensity was measurement by Fiji in reference areas, marked in red for spiral ganglion neurons and in white for supporting cells. The injection of the mice and immunofluorescence staining was performed by Vladan Rankovic and Daniela Gerke (University Medical Center, Göttingen), and micrographs were taken together. 


\subsection{The tetraspanin CD9 enhances exo-AAV production}

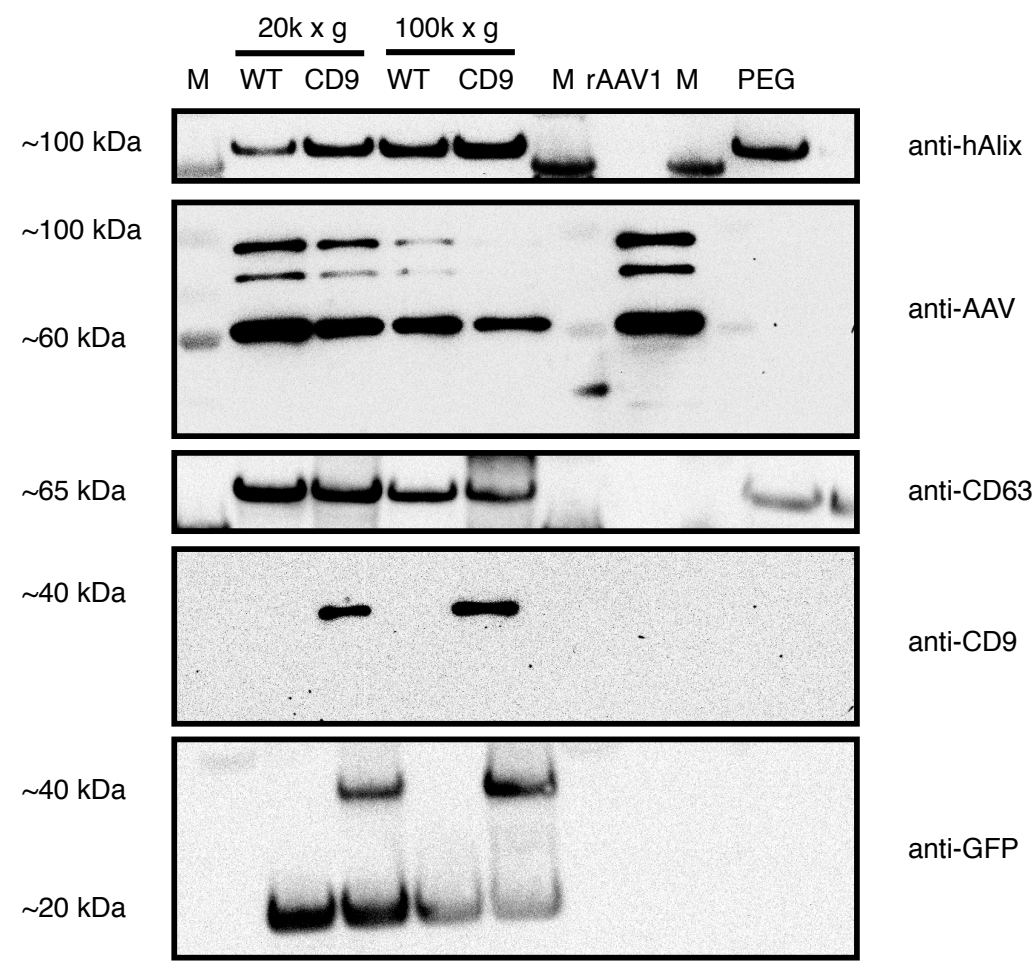

Figure 35: Detection of exosomal markers, rAAVs, and GFP in vesicles of HEKAAV and HEK-AAV-CD9 ${ }_{\text {GFP }}$ cells $20 \mathrm{k} \mathrm{x}$ g and $100 \mathrm{k} \times \mathrm{g}$ pellets of exo-AAV1 production in HEK-AAV and HEK-AAV-CD9 ${ }_{\text {GFP }}$ cells, standard rAAV1, and PEG precipitated vesicles of HEK-AAV cells were precipitated with TCA. Western blot detection of exosomal markers (hAlix, CD63, and CD9) confirmed abundance of exosomes in $20 \mathrm{k} \mathrm{x} \mathrm{g}$ and $100 \mathrm{k} \times \mathrm{g}$ pellet and PEG precipitated vesicles. antiGFP detection confirmed expression of $\mathrm{CD} 9_{\mathrm{GFP}}$ fusionprotein. $\mathrm{M}=$ Marker. The experiment was partially performed by Nicolas Lemus (German Primate Center, Göttingen). Creative commons license CC BY-NC-ND 4.0, modified from Schiller et al. (2018). 
a

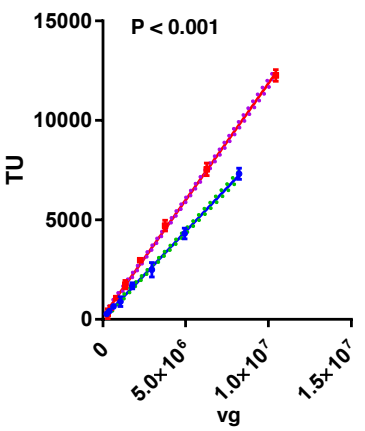

d

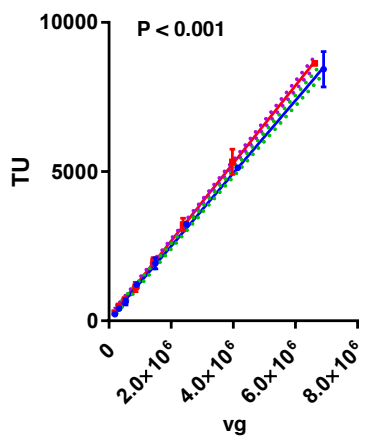

b

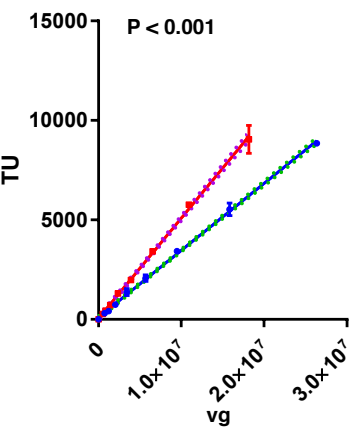

e

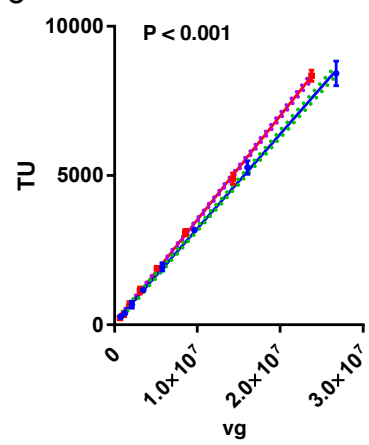

C

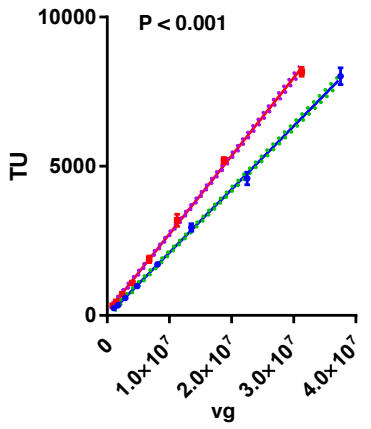

$\rightarrow$ WT

Figure 36: Transduction efficiency of media and 100k pellet of rAAV1 produced in HEK-AAV and HEK-AAV-CD9 ${ }_{\text {GFP }}$ cells A serial dilution of exo-AAV1 media A-C and 100k pellet E,F produced in HEK-AAV and HEK-AAV-CD9 $9_{\mathrm{GFP}}$ cells were applied on HEK cells. The number of GFP transgene expressing cells was evaluated $48 \mathrm{~h}$ post transduction and the values plotted over the applied vector genomes. Linear regression lines (red and blue) were fitted and the P-value (twotailed) calculated to test whether the slopes are significantly different. Error bars indicate the standard deviation of technical triplicates. Creative commons license CC BY-NC-ND 4.0, (Schiller et al., 2018). 


\subsection{The tetraspanin CD9 enhances exosome secretion and lentivirus infectivity}

a
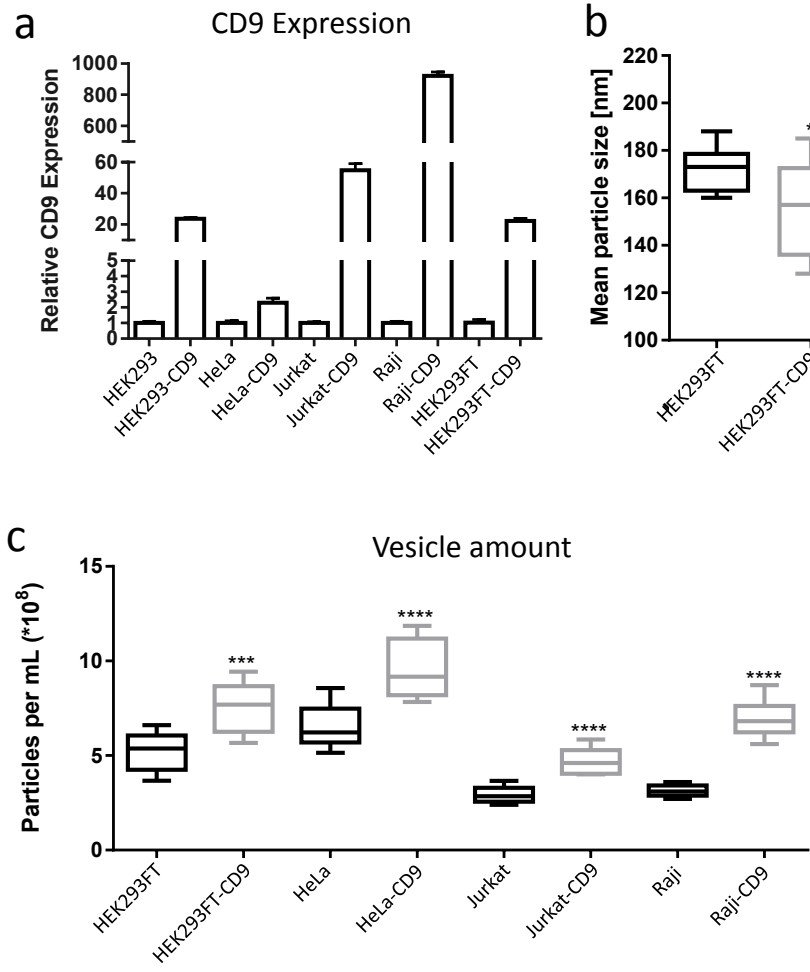

e

LV efficiency HEK293 cells

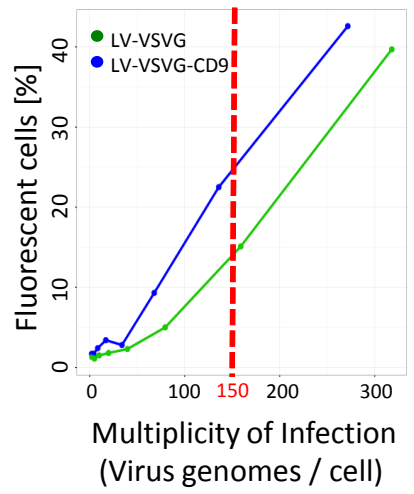

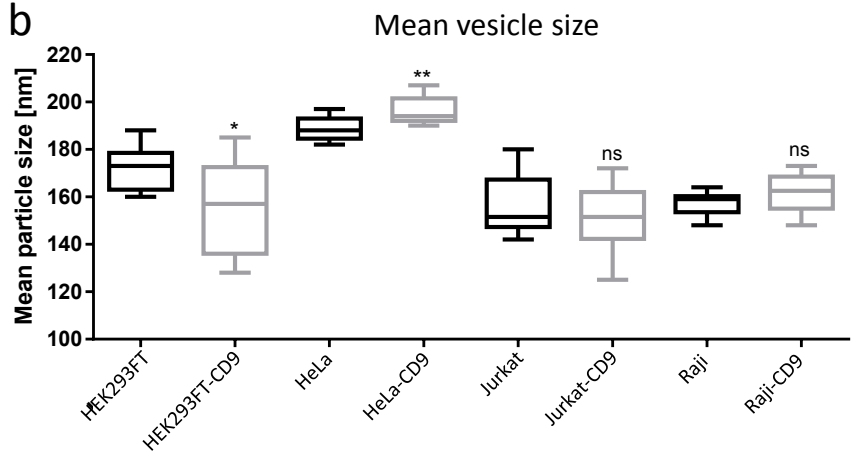

d Exosome amount (30-100nm)

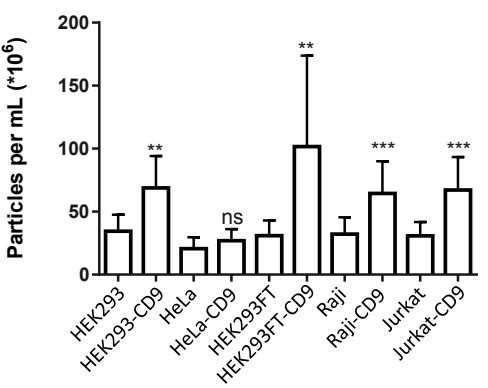

LV efficiency on HEK293 cells (MOI 150)

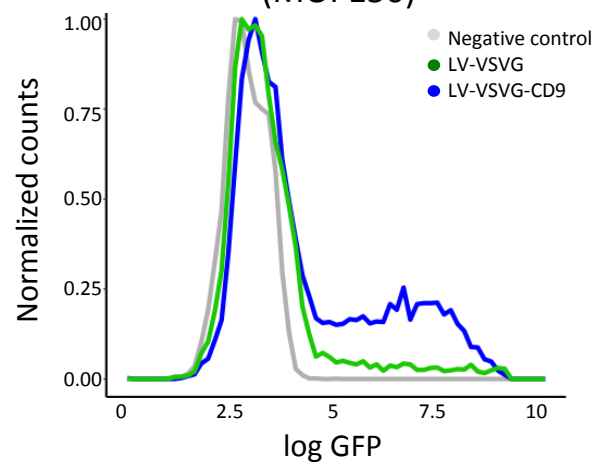

Figure 37: The tetraspanin CD9 enhances exosome secretion and lentivirus infectivity (A) CD9 over-expression in HEK293, HEK293 FT, HeLa, Jurkat and Raji cells was quantified by real-time PCR. (B-D) CD9 over-expression decreased the size of PEG-precipitated vesicles, while it increased the vesicle and exosome amount.

(E) LV-VSVG and LV-VSVG-CD9 were applied on HEK293 cells with an MOI range of 30-300 and the percentage of transgene expressing cells determined by flow cytometry. (F) Density plot of flow cytometry analysis for HEK293 cells transduced with an MOI of 150. Adapted with permissiom from Böker et al. (2017). 


\section{Curriculum Vitae}

\section{EDUCATION AND SCHOLARSHIP}

Sept 2014

- April 2018

Feb- Apr 2018

December 2013

September 2011

\section{PhD candidate}

at the German Primate Center and the Göttingen Graduate School for Neuroscience, Biophysics and Molecular Biology (GGNB)(Supervisor: PhD Jens Gruber)

\section{DFG Grant GSC 226/2}

From the Göttingen Graduate School for Neuroscience, Biophysics, and Molecular Biosciences

\section{M.Sc. Applied and Molecular Botany, University Hamburg}

Title of thesis: Cytokinin homeostasis in Physcomitrella patens (Hedw.) B.S. - Biochemical and molecular analysis of knockout and silencing mutants of the isopentenyltransferase (ipt) gene family (Supervisor: PD Dr. Klaus v. Schwartzenberg)

\section{B.Sc. Biology, Philipps University Marburg}

Title of thesis: Possible synthesis of superoxide in the photocycle of DASHcryptochromes (Supervisor: Prof. Dr. Alfred Batschauer)

\section{CONFERENCES}




\section{PUBLICATIONS}

K. Böker, N. Lemus-Diaz, R. Rinaldi Ferreira, L. Schiller, S. Schneider, and J. Gruber. "The impact of the cd9 tetraspanin on lentivirus infectivity and exosome secretion." Molecular Therapy, 2017.

L. Schiller, N. Lemus-Diaz, R. Rinaldi Ferreira, K. Böker and J. Gruber. "Enhanced production of exosome-associated AAV by over-expression of the tetraspanin CD9." Molecular Therapy Methods $\&$ Clinical Development, 2018 .

\section{EXTRACURRICULAR ENGAGEMENT}

2017

Chair and organiser of the "Göttingen Biotech Symposium", in Göttingen

2017

Organiser of the "Women's Careers and Networks" conference, in Göttingen

\section{WORK EXPERIENCE}

2014- 2018

2014
PhD candidate at Deutsches Primatenzentrum GmbH in the Medical RNA

Biology group

Laboratory assistant in HPLC and GC laboratory at Eurofins Analytik GmbH, Hamburg 